Desplat, Louise (2019) Thermal stability of metastable magnetic skyrmions. $\mathrm{PhD}$ thesis.

Copyright and moral rights for this work are retained by the author

A copy can be downloaded for personal non-commercial research or study, without prior permission or charge

This work cannot be reproduced or quoted extensively from without first obtaining permission in writing from the author

The content must not be changed in any way or sold commercially in any format or medium without the formal permission of the author

When referring to this work, full bibliographic details including the author, title, awarding institution and date of the thesis must be given 


\title{
Thermal stability of metastable magnetic skyrmions
}

\author{
Louise Desplat
}

Submitted in fulfilment of the requirements for the

Degree of Doctor of Philosophy

School of Physics and Astronomy

College of Science and Engineering

University of Glasgow

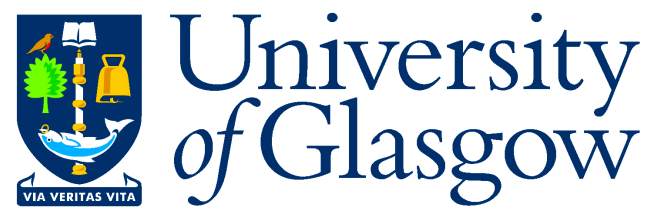

December 2019 


\section{Abstract}

Magnetic skyrmions are two-dimensional, localized, particle-like, topologically nontrivial magnetic spin textures. In recent years, they have attracted a lot of interest as potential candidates for novel spintronics applications. Isolated skyrmions are metastable excitations of the ferromagnetic ground state. They are separated from it by an activation energy, which may be overcome at finite temperature under the effect of thermal fluctuations. In this thesis, we study the thermal stability of metastable magnetic skyrmions on the two-dimensional square lattice, for which we use an atomistic spin model.

This task is firstly carried out via a numerical implementation of Langer's statistical theory for the decay of metastable states. The paths of minimum energy that lead to the skyrmion annihilation are computed via the geodesic nudged elastic band method. The transition state at the barrier top, which is a saddle point (SP), is precisely identified by a climbing image algorithm. We focus on chiral magnetic skyrmions and we look at two types of annihilation mechanisms: collapse, in which the skryrmion progressively shrinks in size until it annihilates, and escape through a boundary. We find that the thermally significant modes are the modes localized to the skyrmion, in contrast to the rest of the collective spin-wave modes, which extend to the entire lattice and contribute weakly. Important variations of the attempt frequency over several orders of magnitude are found, depending on the mechanism and on the value of the external magnetic field. They originate from strong entropic effects which come from the difference in configurational entropy between the metastable skyrmion state and the saddle point. In the cases we studied, the configurational entropy decreases at the SP $(\Delta S<0)$, which results in lowered attempt frequencies, and enhanced skyrmion stability. We refer to this effect as entropic narrowing in the SP region. The strong entropic contribution mainly originates from the skyrmion's internal modes, and is generally more pronounced for collapse mechanisms.

Next, we use forward flux sampling (FFS) to compute skyrmion collapse rates as a function of the applied field, and compare them with the previous results from Langer's theory. This is an important step, because the use of Langer's theory is based on many assumptions. We obtain a good agreement between both methods, thus confirming the strong dependence of the attempt frequency on the external field. While in magnetism, it is common practice to only focus on activation barriers and assume a characteristic value of the prefactor in the gigahertz regime, we conclude that due to a strong entropic contribution, internal energy barriers are not enough in order to correctly predict the lifetime of magnetic skyrmions, and it is essential to also evaluate a rate prefactor.

Lastly, we look at paths to annihilation of first- and second-order skyrmions and antiskyrmions on the frustrated square lattice. Frustrated exchange has been found to arise from interface effects in certain systems where nanoscale interface skyrmions have been observed. We find that, in certain regions of parameter space, the annihilation of skyrmionic solutions no longer occurs through an isotropic type of collapse, and instead involves the injection of the opposite topological charge into the system, by means of the nucleation of merons and antimerons. Alternatively, the second-order (anti)skyrmion may split into a bound (anti)skyrmion pair, which involves no change in the total topological charge. 


\section{Contents}

List of Figures $\quad$ vii

\begin{tabular}{ll|l}
\hline List of Tables & ix
\end{tabular}

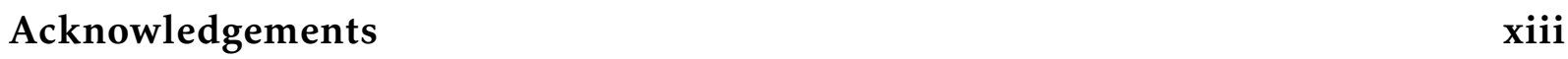

Statement of Originality $\quad$ xv

List of Original Publications $\quad$ xvii

\begin{tabular}{ll}
\hline List of Abbreviations & xix
\end{tabular}

\begin{tabular}{lll}
\hline Introduction & 1
\end{tabular}

2 Topological solitons in magnetic systems 9

2.1 The classical Hamiltonian model . . . . . . . . . . . . . . . 9

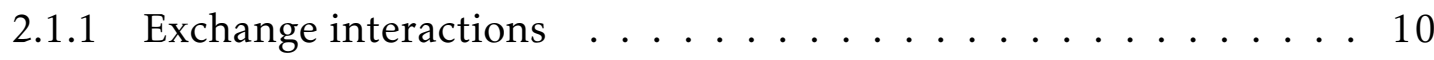

2.1 .2 Zeeman Energy . . . . . . . . . . . . . . . 14

2.1 .3 Magnetic anisotropy ...................... 14

2.1.4 Summary of atomistic and micromagnetics quantities . . . . . . 16

2.2 Ferromagnetic domain walls . . . . . . . . . . . . . 16

2.2.1 Characteristic wall profile . . . . . . . . . . 18

2.2.2 Effect of chiral couplings . . . . . . . . . . . 20

2.2.3 Winding number and topology . . . . . . . . . . . 21

2.3 Magnetic skyrmions . . . . . . . . . . . . . . . . 23

2.3.1 General characteristics . . . . . . . . . . . . 23

2.3.2 Topological charge definition on the atomistic spin lattice . . . . . 25

2.3.3 Chiral skyrmions in non-centrosymmetric magnets . . . . . . 27

2.3.4 Skyrmions in frustrated magnets . . . . . . . . . . . 30

2.3.5 Skyrmion bubbles in dipolar magnets . . . . . . . . . . . 33

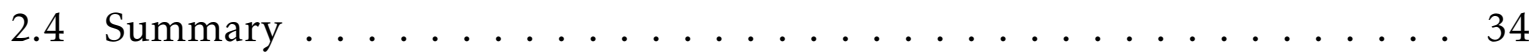


3 Langer's theory and application to magnetic spin systems 39

3.1 Introduction . . . . . . . . . . . . . . . . . . . . . . 39

3.1.1 Preamble: on the theory of Brownian motion . . . . . . . . . 39

3.1 .2 Reaction rate theory . . . . . . . . . . . . . . . . . 42

3.2 Langer's theory in the IHD regime . . . . . . . . . . . . . . . . 44

3.2.1 Framework for the derivation of the rate expression . . . . . . 44

3.2.2 Ratio of energy curvatures: interpretation and the case of Goldstone modes . . . . . . . . . . . . . . . . 50

3.3 Application to magnetic spin systems . . . . . . . . . . . . . . 52

3.3.1 Construction of the energy Hessian in spherical coordinates. . . . 53

3.3.2 Determination of the dynamical prefactor $\lambda_{+} \ldots \ldots \ldots \ldots 54$

3.4 The geodesic nudged elastic band method . . . . . . . . . . . . 57

3.4.1 Projections onto the tangent space to the manifold . . . . . . . . 58

3.4 .2 The GNEB method . . . . . . . . . . . . . . . . . . . . . . 59

3.4 .3 Evaluation of the tangent to the path . . . . . . . . . . 61

3.4 .4 Initialization of the path along geodesics . . . . . . . . . . 61

3.4.5 Search for the first-order saddle point: the CI-GNEB scheme . . . 62

3.4 .6 Variable spring constants . . . . . . . . . . . . . . . 62

3.5 Implementation of Langer's theory on an atomistic spin lattice . . . . . . 64

3.5.1 Atomistic simulations and search for local minima . . . . . . . . . 64

3.5.2 Implementation of GNEB . . . . . . . . . . . . . . . . 64

3.5 .3 Computation of the rate prefactor . . . . . . . . . . 67

3.5 .4 Numerical tests . . . . . . . . . . . . . . . . . . 68

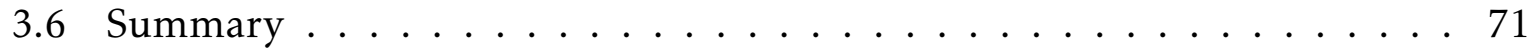

4 Thermal stability of chiral magnetic skyrmions from Langer's theory $\quad 73$

4.1 Introduction . . . . . . . . . . . . . . . . 73

4.2 Simulated system . . . . . . . . . . . . . . . . . . . . 73

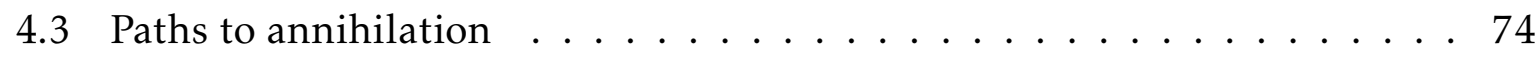

4.3 .1 Collapse mechanisms . . . . . . . . . . . . . . . 74

4.3 .2 Escape through a boundary . . . . . . . . . . . . . 79

4.3.3 Paths to collapse and evolution of the topological charge. . . . . 82

4.4 Transition rates from Langer's theory . . . . . . . . . . . . . . . 82

4.4 .1 Overview . . . . . . . . . . . . . . . . . . 82

4.4 .2 The thermal role of internal eigenmodes . . . . . . . . . . 84

4.4 .3 Entropic contribution to skyrmion stability $\ldots \ldots \ldots$. . . . 90

4.5 Discussion . . . . . . . . . . . . . . . . . . . 97 
5 Skyrmion collapse rate computation via forward flux sampling and comparison with Langer's theory

5.1 Introduction . . . . . . . . . . . . . . . . 101

5.2 Langevin dynamics . . . . . . . . . . . . . . . . . 102

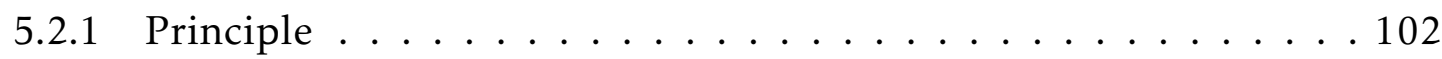

5.2 .2 Numerical implementation . . . . . . . . . . . . . 103

5.2 .3 Numerical tests . . . . . . . . . . . . . . . 105

5.3 The forward flux sampling method . . . . . . . . . . . . 107

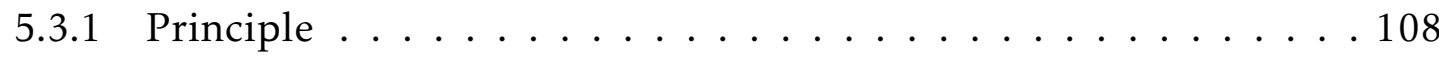

5.3.2 Error estimation and optimization . . . . . . . . . . 110

5.3 .3 Numerical implementation . . . . . . . . . . . . . 113

5.4 Application to skyrmion collapse and comparison with Langer's theory . 114

5.4 .1 Interface definition . . . . . . . . . . . . . 114

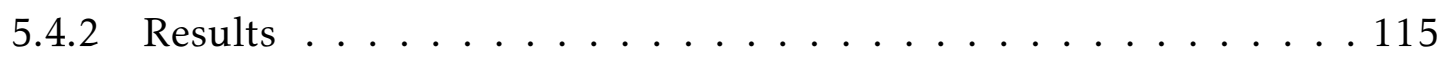

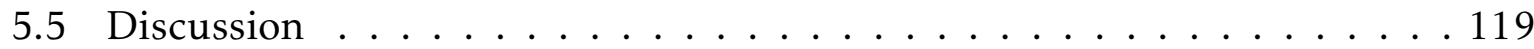

6 Paths to annihilation of first- and second-order (anti)skyrmions under frustrated exchange

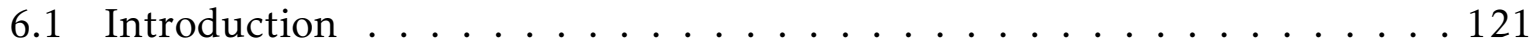

6.2 Coexistence of skyrmion and antiskyrmion solutions . . . . . . . . . 122

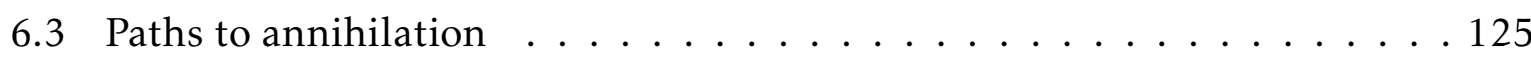

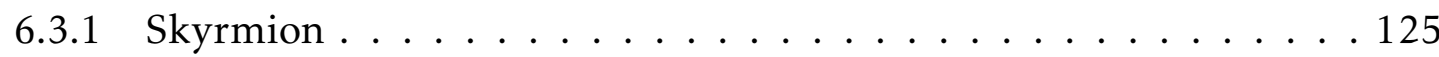

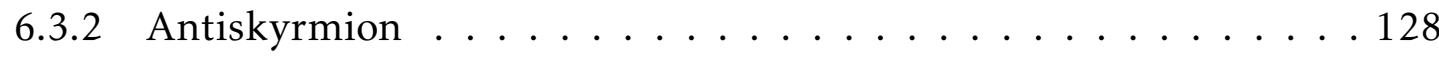

6.3 .3 Second-order skyrmion . . . . . . . . . . . . 130

6.3.4 (Anti)meron nucleation and evolution of the topological charge . 132

6.4 Langevin simulations . . . . . . . . . . . . . . . . . 133

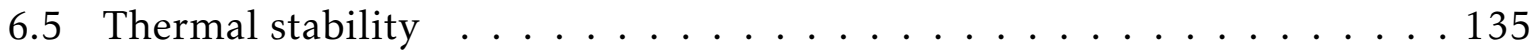

6.6 Discussion . . . . . . . . . . . . . . . . 138

$\begin{array}{lll}7 \text { Conclusion and outlook } & 141\end{array}$

\begin{tabular}{ll}
\hline Appendices & 147
\end{tabular}

A Numerical accuracy in Langer's theory implementation 149

A.1 Checking for numerical noice: influence of the lattice size . . . . . . . . 149

A.2 Influence of the tolerance on the GNEB force . . . . . . . . . . . . . . 149 


\section{List of Tables}

2.1 Symbols, corresponding units and relation between atomistic and micromagnetics quantities. . . . . . . . . . . . . . 17

4.1 Terms of the rate prefactor and total annihilation rate at $T=300 \mathrm{~K}$ for all mechanisms. . . . . . . . . . . . . . . . . . 83

5.1 Theoretical and numerical time average of the component of the magnetization parallel to the anisotropy axis for different temperatures at thermal equilibrium. . . . . . . . . . . . . . . . 105

5.2 Number of interfaces, absolute temperature, number of trial runs, and relative variance of the rate for each value of the applied field. . . . . . 116

5.3 At $b_{z}=0.010$ and for each interface, number of sites inside the skyrmion, and flux through the interface. . . . . . . . . . . . . 116

6.1 Internal energy barrier and rate prefactor for the different annihilation mechanisms. . . . . . . . . . . . . . . . . . . 136

A.1 Terms of the rate prefactor calculated for different number of sites. . . . 150

A.3 Influence of the set tolerance on the GNEB force on the different terms in the collapse rate. . . . . . . . . . . . . . . . . . 150 


\section{List of Figures}

1.1 Spin maps of some topological solitons in magnetism. . . . . . . . . 2

1.2 Néel skyrmion and stereographic projection onto $S^{2}$. . . . . . . . . 4

2.1 Simple cubic lattice and spherical coordinate system. . . . . . . . . . . 11

2.2 Mechanism for interfacial DMI and symmetries of interfacial and bulk

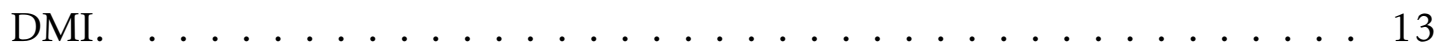

2.3 Bloch and Néel walls and domain wall profile. . . . . . . . . . . . 17

2.4 Chiral and achiral pair of walls and stereographic projection onto $S^{1}$. . . 21

2.5 Spin maps of Néel and Bloch skyrmions and antiskyrmion and stereographic projection of the Néel skyrmion. . . . . . . . . . . . . . 23

2.6 Anatomy of the antiskyrmion spin texture . . . . . . . . . . . . 26

2.7 "Ball in a net" representation of a skyrmion, and elementary triangles on the simple square lattice . . . . . . . . . . . . . . . 26

2.8 Profiles of a chiral skyrmion, a skyrmion stabilized by frustrated exchange, and a skyrmion bubble. . . . . . . . . . . . . . 28

2.9 Examples of skyrmionic solutions stabilized on the $J_{1}-J_{2}-J_{3}$ frustrated square lattice. . . . . . . . . . . . . . . . 30

2.10 Goldstone mode associated with the rotation of helicity, and $E_{\mathrm{ex}}(\mathbf{q})$ in the 2-dimensional Brillouin zone of the square lattice. . . . . . . . . 32

2.11 Examples of skyrmion bubbles. . . . . . . . . . . . . . . . 33

3.1 Example energy surface of a system possessing a metastable local minimum, $A$, and a lower energy minimum, $B$, separated by a first-order

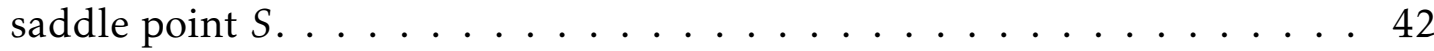

3.2 Schematic dividing surface on the energy landscape through which the current of particles over the barrier is integrated. . . . . . . . . . . 48

3.3 Illustrations of the GNEB method. . . . . . . . . . . . . . . . . . 58

3.4 Iterations of the GNEB method for a single spin reversal. . . . . . . . 63

3.5 Atomistic profiles of a $\pi$-wall and a skyrmion. . . . . . . . . . . . 69 
3.6 Initial state and saddle point of the magnetization reversal in a perpendicular field, and corresponding attempt frequency as a function of reduced field. . . . . . . . . . . . . . . . 70

4.1 Spin maps of the isotropic collapse mechanisms. . . . . . . . . . . 75

4.2 Interpolated energy profiles along the normalized reaction coordinate. . . 76

4.3 Spin maps of the collapse mechanisms in the presence of a defect, and of a second skyrmions. . . . . . . . . . . . . . . 77

4.4 Spin maps of the escape mechanisms. . . . . . . . . . . . . . . 79

4.5 Interpolated energy profile and evolution of the topological charge along the reaction coordinate for collapse mechanisms. . . . . . . . . . . 81

4.6 "Ball in a net" representation of the spin configuration at selected images along the path for collapse mechanisms. . . . . . . . . . . . 81

4.7 Average lifetime of a chiral skyrmion a function of temperature calculated with Langer's theory with respect to all mechanisms. . . . . . . . . . 84

4.8 All energy curvatures at $A$ and $S$ ordered by increasing amplitudes for the case of the collapse of a single skyrmion via mechanism $\mathrm{n}^{0} 1 . \ldots 85$

4.9 First few eigenvalues of the Hessian matrix of each annihilation mechanism and corresponding ratios. . . . . . . . . . . . 86

4.10 Eigenmode profiles for the metastable skyrmion and the saddle points of collapse and escape mechanisms. . . . . . . . . . . . . 87

4.11 Eigenmode profiles for the escape through a curve boundary, the collapse on a defect, and the collapse in the presence of a second skyrmion. . 88

4.12 Calculated change in configurational entropy induced when the system goes to the saddle point as a function of temperature for all mechanisms. $\quad 91$

4.13 Spin maps of the metastable state $A$ and saddle points $S_{1}$ and $S_{2}$ of the skyrmion collapse at different applied field values. . . . . . . . . . 93

4.14 Variation of all the terms entering in the collapse rate as a function of the reduced destabilizing field and for both collapse mechanisms. . . . . 96

5.1 Damped precession of a single spin about the effective magnetic field at zero and finite temperature . . . . . . . . . . . . . 102

5.2 Numerical tests for Langevin dynamics implementation. . . . . . . . . 106

5.3 Illustration of the FFS method. . . . . . . . . . . . . . 108

5.4 Comparison between Langer's theory and FFS for the skyrmion collapse

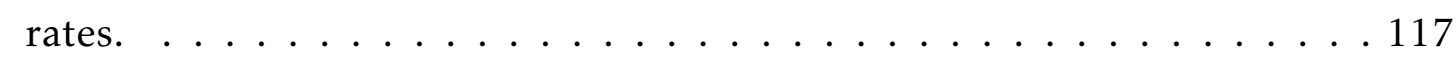

5.5 Examples of spin configurations from FFS stored at the interface(s) at which the order-parameter equates that of a saddle point. . . . . . . 118 
6.1 Regions of existence of metastable first- and second-order (anti)skyrmion solutions as a function of reduced field and DMI. . . . . . . . . . . . . 123

6.2 Spin maps (zoomed) and corresponding topological charge density along the transition path for a skyrmion annihilation. . . . . . . . . . . 126

6.3 For the annihilation of a skyrmion, evolution along the transition path of the internal energy barrier and the topological charge. . . . . . . . . 127

6.4 Spin maps and corresponding topological charge density along the transition path for an antiskyrmion annihilation. . . . . . . . . . . . . . . 129

6.5 For the annihilation of an antiskyrmion, evolution along the transition path of the internal energy barrier and the topological charge. . . . . . 130

6.6 Spin maps and corresponding topological charge density along the transition path for the annihilation of a second-order skyrmion. . . . . . . . . 131

6.7 For the annihilation of a second-order skyrmion, evolution along the transition path of the internal energy barrier and the topological charge. 132

6.8 "Ball in a net" representation of the spin configuration at selected images along the paths to annihilation. . . . . . . . . . . 133

6.9 Probability density distribution of the lifetimes of the antiskyrmion from direct Langevin simulations. . . . . . . . . . . . . . . . . . . 134

6.10 Langevin simulation snapshots of different annihilation mechanisms. . . 135

6.11 Average lifetime of a skyrmion and an antiskyrmion stabilized by exchange frustration a function of temperature calculated with Langer's theory with respect to all mechanisms. . . . . . . . . . . . . 136 


\section{Acknowledgements}

I shall begin by thanking Prof. Robert Stamps who supervised me in Glasgow for the first two years of my PhD. Bob was always kind and patient, and I sincerely enjoyed our scientific discussions. I also acknowledge Prof. Stephen McVitie who took care of the administrative side of my studies in Glasgow after Bob's departure. I thank the Materials and Condensed Matter Physics group in Glasgow for welcoming me for two years. My thanks go to MCMP students of then and now, my 402 officemates, Gavin, Kayla, Matus, Sean, my 318 officemate Bianca, the fourth floor corridor students, Yue, Ray and everyone else, as well as the postdocs, Magnus, John and Trevor for their cheerfulness. I was also glad to get to know the particle physics folks - in particular Donatas. Thank you for being my swimming buddy, and for being the best of friends. Thank you also to Marion and Jess for some great lunch and dinner dates and for the priceless opportunity to complain in French.

A most valuable collaboration over the course of my thesis has been with Prof. Dieter Suess from the University of Vienna, who welcomed me in his group for two consecutive years and almost four months in total. It was really instructional to discuss the stability of skyrmions with him, and I benefited a lot from his input. I later also worked with Dr. Christoph Vogler who guided me with my implementation of forward flux sampling, for which I am grateful. A special thank you also to my Vienna officemate Flo Slanovc for the always fun discussions.

I am deeply indebted to Dr. Joo-Von Kim who kindly welcomed me in his group at the Centre for Nanoscience and Nanotechnology at the University of Paris-Saclay for the last two years of my $\mathrm{PhD}$, and took on the role of my main supervisor. JooVon's meticulous comments, insightful suggestions and warm encouragements were an invaluable help to me. Many thanks as well to everyone in the NOMADE group, my fellow PhD students, Jérôme, Jérémy and Paul, the postdocs, Myoung-Woo, Umesh and Manu, and the students "from the office next to the coffee room", Aurore and Etienne.

As my PhD grant was part of the MAGicSky consortium, I would like to thank the scientific coordinator, Prof. Vincent Cros, and all the members of the consortium who provided great learning opportunities during the project meetings. In particular, I thank Dr. Bertrand Dupé who welcomed me at the Christian Albrechts University of 
Kiel for a week, and who also offered me some valuable suggestions during his visits to Paris. I extend my sincere thanks to Prof. Stefan Blügel for inviting me to give a seminar in Jülich, and for the great discussions I was able to have with him and the other members of the Peter Grünberg Institute. In particular, I thank Dr. Gideon Müller for some insightful discussions. I also thank Dr. Pavel Bessarab from the University of Iceland for his useful remarks and for kindly answering the questions I sent him via email. Thank you as well to Dr. Gerard Duff, formerly from University College Dublin.

I thank my thesis Examiners, Dr. Donald MacLaren from the University of Glasgow, and Prof. Gino Hrkac from the University of Exeter, as well as the Convener, Dr. Laura Clark.

Lastly, I thank my family for their support. I am particularly grateful to my sister-inlaw Elise for always offering a friendly and sympathetic ear in difficult times. Last but not least, I endlessly thank my mother for her unwavering support and encouragements through the years.

This PhD was funded by the Horizon 2020 Framework Programme of the European Commission, under Grant agreement No. 665095 (MAGicSky), and the Agence Nationale de la Recherche under Contract No. ANR-17-CE24-0025 (TOPSKY). 


\title{
Statement of Originality
}

\author{
University of Glasgow
}

\section{College of Science and Engineering}

Name: Louise Desplat

Registration Number:

I certify that the thesis presented here for examination for a PhD degree of the University of Glasgow is solely my own work other than where I have clearly indicated that it is the work of others (in which case the extent of any work carried out jointly by me and any other person is clearly identified in it) and that the thesis has not been edited by a third party beyond what is permitted by the University's PGR Code of Practice.

The copyright of this thesis rests with the author. No quotation from it is permitted without full acknowledgement.

I declare that the thesis does not include work forming part of a thesis presented successfully for another degree.

I declare that this thesis has been produced in accordance with the University of Glasgow's Code of Good Practice in Research.

I acknowledge that if any issues are raised regarding good research practice based on review of the thesis, the examination may be postponed pending the outcome of any investigation of the issues.

Signature:

Date: 


\section{List of Original Publications}

1. L. Desplat, D. Suess, J.-V. Kim, and R. L. Stamps. Thermal stability of metastable magnetic skyrmions: Entropic narrowing and significance of internal eigenmodes. Physical Review B, 98:134407, 2018

2. L. Desplat, J.-V. Kim, and R. L. Stamps. Paths to annihilation of first-and secondorder (anti) skyrmions via (anti) meron nucleation on the frustrated square lattice. Physical Review B, 99(17):174409, 2019

3. L. Desplat, C. Vogler, J.-V. Kim, R. L. Stamps, and D. Suess. Path sampling for lifetimes of metastable magnetic skyrmions and direct comparison with Kramers' method. arXiv:1905.04110, 2019 


\section{List of Abbreviations}

CI climbing image

DFT density functional theory

DMI Dzyaloshinskii-Moriya interaction

FFS forward flux sampling

FM ferromagnetic

FPE Fokker-Planck equation

GNEB geodesic nudged elastic band

IHD intermediate-to-high damping

im. image

LLG Landau-Lifshitz-Gilbert

MEP minimum energy path

PMA perpendicular magnetic anisotropy

SOC spin-orbit coupling

SP saddle point

SP-STM spin-polarized scanning tunneling microscopy

SS spin spiral

TEM transmission electron microscopy

TST transition state theory 
"So comes snow after fire,

and even dragons have their endings!"

J.R.R. TOLKIEN,

The Hobbit or There And Back Again. 


\section{Chapter 1}

\section{Introduction}

Historically, a soliton phenomenon was first described in 1834 by civil engineer John Scott Russell, in the Edinburgh and Glasgow Union Canal in Scotland [4]. He observed a propagating water wave with great stability that travelled through a large distance without loss of shape or velocity, and named it a "wave of translation". He later reproduced the phenomenon in a water tank and, apart from their stability, described the following properties of solitons: (i) their speed depends on their size and their width on the water depth; (ii) waves do not merge, and instead a bigger and faster wave travels through a smaller and slower one; (iii) a wave too big for the water depth separates into two smaller waves. More generally, solitons correspond to localized solutions to a nonlinear wave equation, and are the result of a balance between dispersive effects and nonlinearities [5]. One example is the sine-Gordon equation, which we give in $(1+1)$ dimensions for the scalar field $\phi(x, t)[6]$,

$$
\partial_{t}^{2} \phi-\partial_{x}^{2} \phi+\sin \phi=0
$$

Eq. (1.1) admits solitons as some of its solutions. Solitons such as waves in water are intrinsically dynamical objects, and simply relax back to the uniform state in the static case. By contrast, in systems with strong couplings between the microscopic entities, as is the case in magnetic materials, a class of solitons known as topological solitons can be statically stable due to topological constraints. Examples of topological solitons in magnetism, also known as topological defects, include domain walls, vortices, skyrmions, and bubbles [7]. We show some typical spin maps of the aforementioned structures in Fig. 1.1 .

The term "skyrmion" originated from baryonic field theories, and initially designated a type of solitonic solutions derived by Skyrme [8] within his model for low energy dynamics between mesons and baryons. The term "magnetic skyrmion" was later introduced into the field of condensed matter physics to designate so-called "chi- 


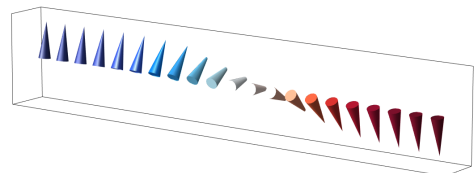

(a)

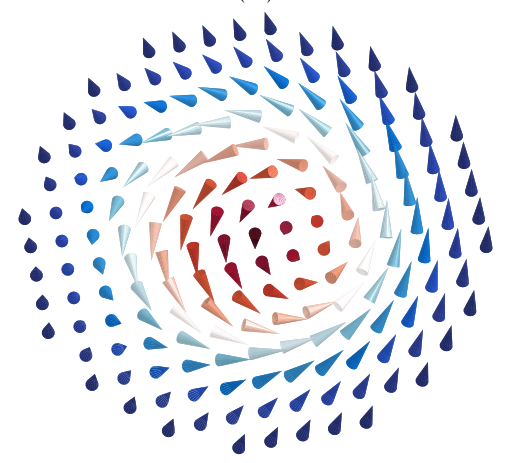

(c)

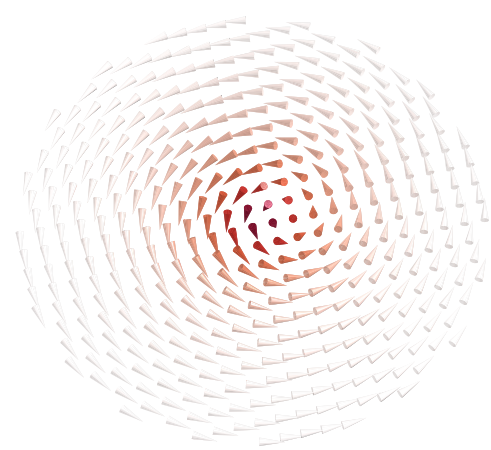

(b)

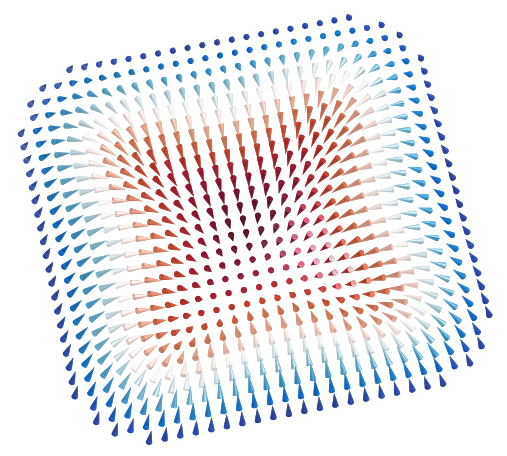

(d)

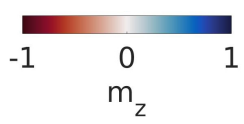

Figure 1.1: Spin maps of some topological solitons in magnetism. (a) A (Bloch) domain wall in a chain of spins. The magnetization undergoes a $\pi$-rotation, from one out-ofplane orientation to the other. (b) A magnetic vortex. The magnetization goes from out of plane in the centre, to in plane far from the centre, and thus undergoes a total $\pi$-rotation along a radial cross-section. (b) A (Bloch) magnetic skyrmion. The magnetization rotates from one out-of-plane orientation far from the centre, to the opposite out-of-plane orientation in the centre, resulting in a total $2 \pi$-rotation along a radial cross-section. (c) A bubble domain. Similar to a skyrmion, the magnetization rotates by $2 \pi$ along a radial cross section, but the profile is different, with a more spatially extended core. It follows that domain walls and vortices carry a half-integer topological charge, while skyrmions and bubbles carry an integer charge.

ral magnetic vortices" theoretically studied by Bogdanov et al. in the 1990s [9-11]. Magnetic skyrmions are two-dimensional, non-collinear magnetic spin textures which are localized in space (Fig. 1.2a). Most commonly, the existence of skyrmion solutions in magnets is made possible by the antisymmetric Dzyaloshinskii-Moriya interaction (DMI) [12,13] which favours non-collinear spin textures in systems lacking inversion symmetry. DMI exists in non-centrosymmetric magnets, and can also be induced in centrosymmetric crystals if the symmetry is broken by an external mechanism. In particular, interfacial DMI emerges in thin magnetic films due to the breaking of the inversion symmetry at the interface [14,15]. Magnetic skyrmions stabilized by the DMI are known as chiral magnetic skyrmions, because the DMI selects a preferred chirality - or 
handedness - of the spin texture. However, they may be stabilized in centrosymmetric magnets where long-range dipole-dipole couplings compete with the dominant shortrange isotropic exchange term (skyrmion bubbles) [16-21], or by introducing frustration in the exchange [22-27].

A skyrmion lattice was first detected in the bulk of the B20 chiral magnet $\mathrm{MnSi}$ in reciprocal space, via neutron scattering [28]. Shortly after, Yu et al. reported the first real space observation of a skyrmion lattice in a thin film of $\mathrm{Fe}_{0.5} \mathrm{Co}_{0.5}$ by means of Lorentz transmission electron microscopy [29]. These observations initiated a strong regain of interest in the community, and the existence of skyrmions was then confirmed in many other types of materials [30-32], including thin films [33, 34]. Similarly to domain walls [17], which have been used as information carriers in racetrack memories [35], magnetic skyrmions can be moved with spin torques associated with spin polarized currents [36, 37], and have therefore been envisioned for potential spintronics applications in data storage and logic devices [38-42]. Contrary to individual $\pi$ domain walls, skyrmions are localized in two dimensions, and therefore leave the magnetization field intact far away from the centre. Their equilibrium sizes are determined by the strength of the different competing energy terms, and it has been proposed that skyrmion with sizes down to the nanometer scale may be stabilized by adequately tuning material parameters [43, 44]. Additionally, they exhibit a particle-like behaviour which finds its root in the topology, and is independent of the underlying stabilizing mechanism [24, 45]. For technological applications, it it necessary to design structures with great stability and robustness against external effects and deformations. Because they are classified as "topologically stable", and for all the reasons mentioned above, skyrmions are compelling candidates to be used as movable information bits in future spintronics devices.

Potential stability arguments for skyrmions are naturally found in the concepts of topology (or homotopy), and winding number. Topologically nontrivial textures may be seen as a knot in a rope, which cannot be untied without using the extremities, or by cutting the rope. The stability of such textures thus stems from their resistance against deformation. Configurations are said to be topologically (or homotopically) equivalent if it is possible to continuously transform one into the other without introducing point defects [7]. Topological stability arises if a structure is topologically nontrivial, and therefore cannot be continuously deformed into the topologically trivial uniform state. Mutually deformable configurations form a class of mappings, $f: S^{n} \rightarrow X$ of the $n$-sphere $S^{n}$ (the "base manifold") into the order-parameter space $X$ (the "target manifold"), and yield equivalence - or homotopy - classes $\pi_{n}(X)$. The winding number, or degree of mapping, $\operatorname{deg} f$, counts the number of times the target manifold $X$ 


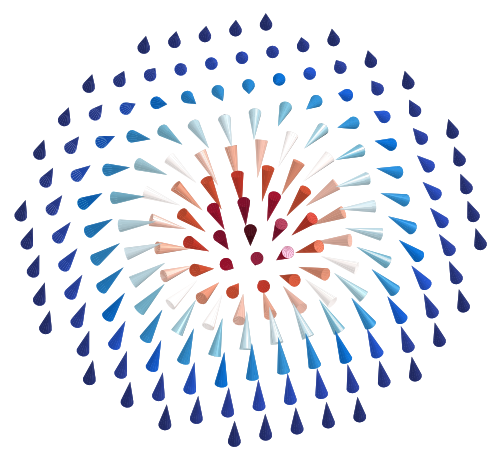

(a)

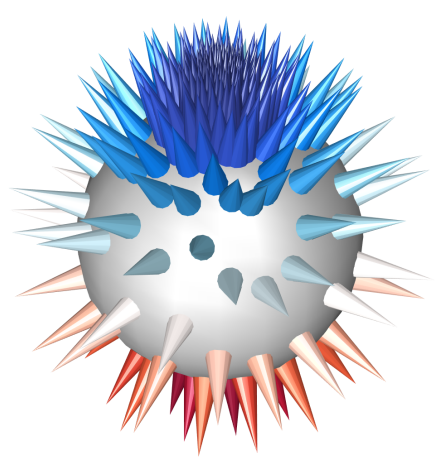

(b)

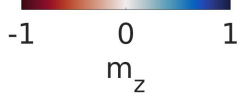

Figure 1.2: (a) Néel "hedgehog" skyrmion and (b) stereographic projection onto the 2-sphere $S^{2}$.

wraps around the base manifold $S^{n}$. Formally, it provides a bijection of the homotopy classes into the integers $\mathbb{Z}$, and may be used to classify homotopy classes. That is to say, configurations with different winding numbers do not pertain to the same homotopy class and are therefore not topologically equivalent, and so they cannot be continuously transformed into each other.

In magnetism, we are concerned with the mappings provided by the magnetization field $\mathbf{m}(\mathbf{r})$. In an infinite system, two magnetic configurations are said to be topologically (or homotopically) equivalent if it is possible to continuously transform one into the other without having to overcome an infinite energy barrier [45]. The uniformly magnetized state, also referred to as the ferromagnetic (FM) state, has a vanishing winding number, which makes it topologically trivial. On the other hand, topological defects such as magnetic skyrmions are topologically non-trivial. In isotropic spin systems with two spatial dimensions, the order parameter space is the 2-sphere $S^{2}$. As we assume that the magnetization takes a constant value at infinity, the base manifold is the physical space $\mathbb{R}^{2}$ with infinity compactified to a point, which may be stereographically projected onto $S^{2}$ (Fig. 1.2b). We then have the map $f: S^{2} \rightarrow S^{2}$, which corresponds to the homotopy class $\pi_{2}\left(S^{2}\right)=\mathbb{Z}$. Skyrmions possess an integer winding number, and consequently cannot be continuously transformed into the uniform state. For this reason, they are classified as "topologically stable". Nevertheless, this classification corresponds to an idealized viewpoint. The notion of topological stability implies that the magnetization varies slowly with respect to the lattice, and thus may be seen as a continuous vector field $\mathbf{m}(\mathbf{r}, t)$. In physical systems, the energy barriers are not infinite [45] because (i) the magnetization is defined on a discrete lattice and thus may, in some cases, vary on the scale of the lattice parameter, and (ii) samples are finite in size and so topological defects may be injected or ejected at the edges. The finite energy 
barriers imply that isolated skyrmions which exist as excitations of the ferromagnetic ground state are, in fact, metastable states with a finite lifetime, $\tau$. At non-zero temperature, there is a finite probability that they eventually annihilate under the effect of thermal fluctuations, and the uniformly magnetized configuration is recovered. In order for skyrmions to be used in applications, one must be able to, firstly, predict their average lifetime, and, secondly, understand it in order to potentially tune it to achieve high stability in room-temperature conditions. For data storage applications, the technical requirement is an average lifetime of ten years at room temperature [46]. We are thus faced with the problem of estimating the rate at which a metastable state decays.

The rate of decay of metastable states is an ubiquitous problem in physics. Thermal activation processes across an energy barrier are found within fields as diverse as solid state physics (Josephson tunnelling junctions), chemical reactions, electrical circuit theory (phase-locked loops), laser physics, and magnetization switching in ferromagnets [47,48]. It can be described as originating from the nucleation and growth of some characteristic disturbance within the system [49]. Amongst the most well known examples of this phenomenon, one can cite the case of a supersaturated vapour, which can exist as a metastable state for a long time, until the nucleation of a large enough water droplet leads to the condensation of the entire system. Similarly, in a ferromagnet, a magnetization reversal is initiated by a cluster of reversed spins. The rate of such thermally activated processes may be generally described by the Arrhenius law [50],

$$
k=f_{0} e^{-\Delta E / k_{B} T},
$$

in which $\Delta E$ is the internal energy barrier, and the pre-exponential factor $f_{0}$, commonly referred to as the attempt frequency, corresponds to a fundamental fluctuation rate. Eq. (1.2) laid the foundations for modern reaction rate theory. The mean lifetime of a metastable state may be taken as the inverse of its decay rate, $\tau=k^{-1}$. To calculate the rate, the following approaches are possible. On the one hand, a treatment of the different terms on the right-hand side of Eq. (1.2) may be achieved by applying a form of reaction rate theory [47, 50]. This requires the evaluation of the activation energy $\Delta E$, and of the rate prefactor $f_{0}$. Alternatively, a total rate $k$ may be computed through numerical integration of the system dynamics. In the rest of this discussion, we focus on magnetic systems.

Historically, the rate of magnetization reversal in single-domain magnetic particles has been extensively studied [51]. Since their magnetization can be described by a macrospin with only two degrees of freedom, they are well suited for an analytical treatment. In the case of inhomogeneous transitions, thermally activated magnetization reversals were treated analytically by Braun in nanowires [52], by Loxley 
and Stamps in soft/hard nanowires [53], and by Visscher et al. in nanowires with graded anisotropy [54]. For more complex systems, one typically has to rely on numerical methods. A systematic application of reaction rate theory may be realized in two main steps. First, a path finding scheme, such as the nudged elastic band method (NEB) [55-59] or the string method [60,61], may be used to relax minimum energy paths separating two energy minima, and identify the saddle point (SP) along the path. A minimum energy path constitutes a path of highest statistical weight, and therefore corresponds to a particular mechanism of a given transition. After that, the rate prefactor needs to be evaluated. This calculation tends to be tedious, and has rarely been carried out [1, 62, 63]. Instead, estimating the stability of magnetic structures is often synonymous with evaluating internal energy barriers, while assuming a typical value of the prefactor in the gigahertz range [64-67].

An alternative approach lies in the numerical integration of the dynamical equations of motion at finite temperature, i.e., Langevin simulations. In magnetic spin systems, the magnetization dynamics is governed by the Landau-Lifshitz-Gilbert (LLG) equation. For structures with lifetimes well above the nanosecond range, transitions become rare events on the timescale of the simulation, due to the small timesteps ( $d t \sim$ $10^{-15} \mathrm{~s}$ ) required to resolve the precessional dynamics [68]. Therefore, the "bruteforce" method that relies on direct Langevin simulations becomes unrealistic. One may resort to temperature-accelerated dynamics [69,70], but such simulations reproduce the low-temperature behaviour only under the assumption that reaction rate theory holds. For structures whose lifetime extends to the scale of years, temperatures well above the Curie temperature are required in order to obtain the necessary acceleration, and therefore a problem of consistency arises if one aims to study ordered spin states. In this case, a better suited class of methods are path sampling methods [71-77]. In particular, forward flux sampling (FFS) [74-77] may be used to compute rate constants of rare events even in systems that are out of equilibrium. Forward flux sampling was shown to be much more computationally efficient than brute force simulations [75, 77]. It has, for instance, been successfully applied by Vogler et al. [78,79] to obtain switching rates in magnetic microstructures.

The aim of this thesis is to study the thermal stability of metastable magnetic skyrmions in two-dimensional systems. This problem is difficult to solve analytically and requires numerical tools. In micromagnetics simulations, where the magnetization is treated as a continuous vector field, and within a three-dimensional physical space, skyrmions typically decay via the formation of a Bloch point, a topological singularity where the continuity of magnetism is broken [80]. While Bloch points do not strictly exist in two dimensions, equivalent processes to the three dimensional ones have been 
reported (see discussion in [80] and subsequent refs.). The use of atomistic simulations is therefore necessary in order to avoid a mesh-size dependency of the activation rates [81]. The annihilation rates are thus calculated on an atomistic spin lattice via two main methods. The first one is a form of reaction rate theory which corresponds to Kramers' method [82] for a multidimensional phase-space in the intermediate-to-high damping (IHD) regime, namely Langer's statistical theory of the decay of metastable states [49]. The second one is forward flux sampling.

The present thesis is organized as follows. In Chapter 2, we give the different terms in the magnetic Hamiltonian, and we discuss in detail two types of topological solitons: ferromagnetic walls and magnetic skyrmions. In Chapter 3, we present Langer's theory and how it is applied to magnetic spin systems. We then provide details of the different building blocks in the implementation of Langer's theory on the atomistic spin lattice. In particular, we present the geodesic nudged elastic band (GNEB) method, which allows us to relax minimum energy paths on the energy surface. After that, Langer's theory is applied to obtain annihilation rates of metastable magnetic skyrmions via collapse and escape processes in Chapter 4. In Chapter 5, we expose the principles behind the forward flux sampling method, and we apply it to compute collapse rates of metastable magnetic skyrmions in an infinite system. The obtained results are subsequently compared with those from Langer's theory. Next, in Chapter 6 , we introduce frustration in the exchange and, in that context, we look at paths to annihilation of skyrmionic solutions in frustrated systems, and their stability is discussed. A general discussion of the results and some perspectives are, lastly, given in Chapter 7 . 


\section{Chapter 2}

\section{Topological solitons in magnetic}

\section{systems}

In this chapter, we begin by introducing the different terms in the classical Hamiltonian model for ferromagnets in Sec. 2.1. In Sec. 2.2, we give some elements of theory for ferromagnetic domain walls, which we use to introduce the key notions of winding number and topology. Finally, in Sec. 2.3, we define magnetic skyrmions and present the different types of systems in which they can be stabilized.

\subsection{The classical Hamiltonian model}

A ferromagnet is characterized by a spontaneous magnetization associated with long range magnetic ordering. This originates from exchange interactions between atoms, which favour parallel orientations of neighbouring atomic spins, and constitute the dominant energy contribution in magnetic materials. The different terms in the energy of a magnetic system describe the interaction of the magnetic moments with an external field, the crystal lattice, as well as interactions with the other magnetic moments. These consist of short-range exchange interactions originating from quantum mechanics, and long-range magnetostatic interactions. Equilibrium magnetization structures are found by minimizing the total energy of a system, and correspond to the case when the total effective field exerts no torque on the magnetic vectors.

We consider an ensemble of $N$ atoms on a lattice with lattice constant $a$ (Fig. 2.1a). Each atom localized at site $i$ carries a net magnetic moment that we write in the form of a classical vector $\mathbf{m}_{i}=\mathbf{M}_{i} / M_{s}$, in which $M_{s}$ is the saturation magnetization and $\mathbf{m}_{i}$ is a unit vector. The magnetic moment is linked to the atomic spin $\mathbf{S}_{i}$ by the relation $\mathbf{M}_{i}=-g \mu_{B} \mathbf{S}_{i}$ [83]. In the previous expression, $g$ is the Landé factor and $\mu_{B}$ is the Bohr magneton. In the rest of this thesis, we use the words spins and magnetic moments 
interchangeably. The total energy of the assembly of spins in its atomistic form is

$$
E=E_{\mathrm{ex}}+E_{\mathrm{DM}}+E_{Z}+E_{\mathrm{an}}
$$

where $E_{\mathrm{ex}}$ is the isotropic exchange energy, $E_{\mathrm{DM}}$ is the DMI exchange energy, $E_{Z}$ is the Zeeman energy, and $E_{\text {an }}$ is the anisotropy energy. Note that we neglect dipolar couplings, although they are discussed later in this chapter.

Within the framework of micromagnetics theory [84], we assume only small angular variations between the orientations of neighbouring spins, which allows us to write the magnetization as a continuous vector field, $\mathbf{m}=\mathscr{M} / \mathscr{M}_{s}$, with norm $\mathscr{M}_{s}$. In the rest of this work, micromagnetics quantities are symbolized with cursive letters - with the exception of the spin stiffness, $A$ - while regular symbols represent atomistic quantities. The micromagnetics Hamiltonian reads

$$
\mathscr{E}=\mathscr{E}_{\mathrm{ex}}+\mathscr{E}_{\mathrm{DM}}+\mathscr{E}_{Z}+\mathscr{E}_{\mathrm{an}}
$$

where the indices have the same meaning as in Eq. (2.1).

In the rest of this section, we introduce the different terms in Eq. (2.1) within the classical Heisenberg model, as well as their corresponding expressions in Eq. (2.2) from micromagnetics theory. We give particular emphasis to the case of thin magnetic films with perpendicular magnetic anisotropy (PMA), as this is a category of systems which is currently the most envisioned for spintronics applications, as well as the type of system that was studied in the present thesis.

Let us define here the spherical coordinate system $(1, \theta, \phi)$ (Fig. 2.1b) which we shall use throughout this thesis. $\theta$ is the polar angle of the magnetization with the $z$-axis, and $\phi$ is the corresponding azimuth angle in the $x y$-plane, so that the unit magnetic vector $\mathbf{m}=\left(m_{x}, m_{y}, m_{z}\right)$ may be expressed as

$$
\mathbf{m}=(\cos \phi \sin \theta, \sin \phi \sin \theta, \cos \theta) .
$$

\subsubsection{Exchange interactions}

\section{Isotropic Heisenberg exchange}

The isotropic exchange interaction originates from the overlap of electronic orbitals and was first proposed by Heisenberg in 1928 [85] to interpret the large molecular field observed in ferromagnets. A year later, Dirac [86] showed that the interaction 


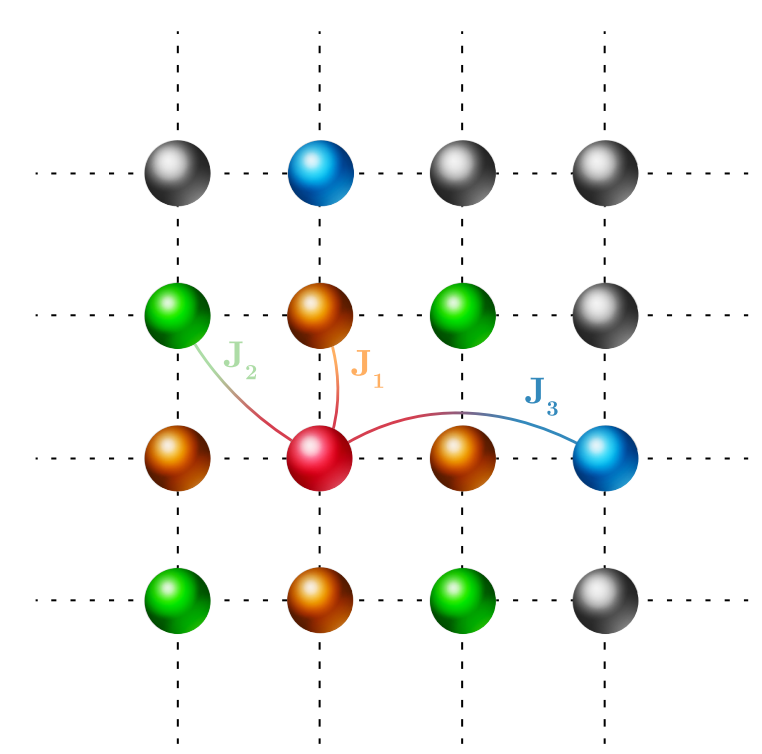

(a)

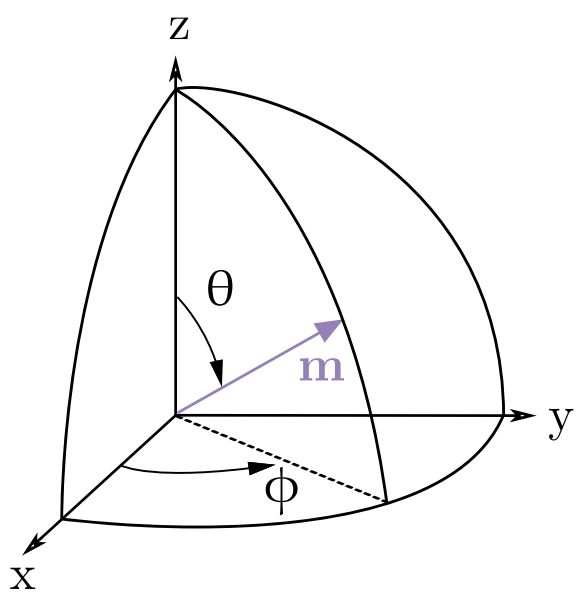

(b)

Figure 2.1: (a) A simple cubic lattice, where $J_{1}$ is the isotropic exchange coupling between nearest neighbours, $J_{2}$ is between next-nearest neighbours, and $J_{3}$ is between next-next-nearest neighbours. Unless the exchange is frustrated, we restrict ourselves to nearest-neighbour interactions. (b) The spherical coordinate system used throughout this thesis.

energy between two neighbouring sites $i, j$ can be expressed in its atomistic form as $-2 J_{i j} \mathbf{m}_{i} \cdot \mathbf{m}_{j}$, where $J_{i j}$ is the exchange integral between sites $i$ and $j$. It decreases rapidly as a function of distance, which in turn explains the short-range nature of the interaction. Isotropic exchange is referred to as ferromagnetic if it favours parallel spins, i.e., if $J_{i j}>0$, and antiferromagnetic if $J_{i j}<0$, which favours anti-parallel spins. The interaction is symmetric upon interchanging $i$ and $j$, and isotropic as it is independent of the direction of the change relative to the magnetization direction. The total isotropic exchange energy of a system of $N$ interacting spins is expressed as

$$
E_{\mathrm{ex}}=-\sum_{<i j>} J_{i j} \mathbf{m}_{i} \cdot \mathbf{m}_{j}
$$

in which the summation is done over pairs of neighbouring spins $\langle i j\rangle$, with $i, j=$ $1 \ldots N$ and $i \neq j$. For the special case where $J_{i j}$ is constant for all pairs $\langle i j\rangle$, we write $J_{i j}=J_{\mathrm{ex}}=J_{1}$ if $i$ and $j$ are nearest neighbours, $J_{i j}=J_{2}$ if they are next-nearest neighbours, etc (see Fig. 2.1a).

In the continuum limit, isotropic exchange may be expressed as

$$
\left.\mathscr{E}_{\mathrm{ex}}=\int \mathrm{d} V A\left(\left(\nabla m_{x}\right)^{2}+\left(\nabla m_{y}\right)^{2}+\left(\nabla m_{z}\right)^{2}\right)\right) \text {, }
$$


where $\nabla=\partial_{x} \mathbf{e}_{x}+\partial_{y} \mathbf{e}_{y}+\partial_{z} \mathbf{e}_{z}$ is the Nabla operator, $\mathrm{d} V$ is an elementary volume, and $A=J_{\mathrm{ex}} n / a$ is the exchange stiffness in units of energy per unit length $(\mathrm{J} / \mathrm{m})$, with $n$ the number of atoms per unit cell. In all that follows, we restrict ourselves to the simple cubic lattice shown in Fig. 2.1a, for which $n=1$.

\section{The Dzyaloshinksii-Moriya Interaction (DMI)}

The Dzyaloshinskii-Moriya interaction, or DMI, is an antisymmetric, anisotropic exchange term which arises in low symmetry crystals due to spin-orbit coupling (SOC). In the Heisenberg model, the total DMI energy of a system of $N$ interacting spins is expressed as

$$
E_{\mathrm{DM}}=-\sum_{<i j>} \mathbf{D}_{i j} \cdot\left(\mathbf{m}_{i} \times \mathbf{m}_{j}\right) .
$$

The DMI is typically a few orders of magnitude lower than isotropic exchange. In Eq. (2.6), $\mathbf{D}_{i j}$ is known as the Dzyaloshinskii vector. Its orientation depends on the symmetries of the crystal lattice and it vanishes if a centre of inversion is present between sites $i$ and $j$. It follows from Eq. (2.6) that the energy contribution associated with the DMI is minimum when neighbouring spins are oriented normal to each other, with a given sense of rotation determined by the orientation of $\mathbf{D}_{i j}$. As a consequence, the DMI tends to rotate spins in competition with the other interactions to create a variety of non-collinear magnetic configurations, such as helicoids and skyrmions. It also lifts degeneracies by favouring a particular chirality of existing spin textures, as is the case for magnetic vortices and domain walls. This interaction was first theorized by Dzyaloshinskii [12] who, using symmetry considerations, showed that a combination of low symmetry and spin-orbit coupling gives rises to an antisymmetric term in the exchange. Moriya [13] then identified a microscopic mechanism which accounts for the presence of such a term in systems with spin-orbit coupling. DMI occurs in crystals with low symmetry such as non-centrosymmetric magnetic crystals - i.e., crystals lacking inversion symmetry. One of the first observations of its effect was weak ferromagnetism in antiferromagnetic compounds such as $\alpha-\mathrm{Fe}_{2} \mathrm{O}_{3}$ and $\mathrm{CrF}_{3}$, in which a net ferromagnetic moment emerges due to a small misalignment of the sublattice magnetization caused by antisymmetric exchange [13]. It was later found in chiral bulk magnets such as MnSi, in which a helical phase was observed at low temperature [87, 88.

DMI can also be induced in centrosymmetric crystals if the symmetry is broken by an external mechanism. This effect is weak in bulk materials, but becomes important in small magnetic structures such as thin films, multilayers, nanowires and nanodots. In thin films, the broken inversion symmetry at the surface is responsible for the emergence of interfacial DMI $[14,15,34,89]$. Strong DMI is these systems is engineered by 


\section{Strong SOC material}

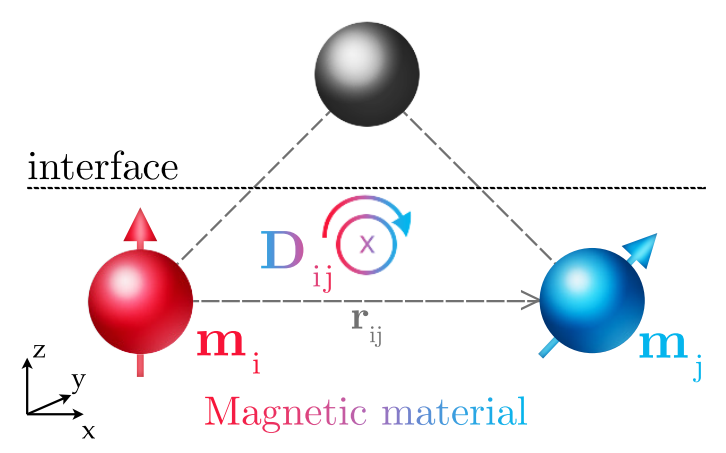

(a)

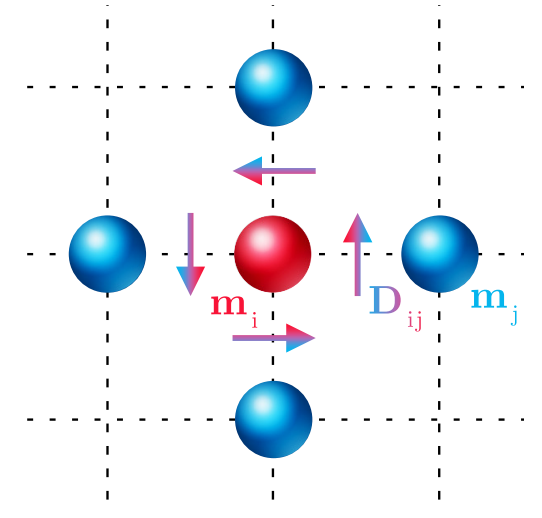

(b)

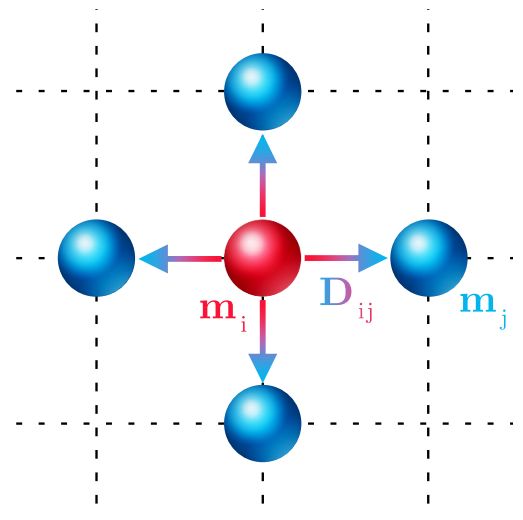

(c)

Figure 2.2: (a) DMI induced by the vertical breaking of the inversion symmetry along $z$ at an interface between a magnetic material and a material exhibiting strong spinorbit coupling. (a) and (b) show different symmetries of the DMI in the $x y$-plane on a simple square lattice. (b) In the case where the DMI is induced at an interface via breaking of the inversion symmetry along $z$, the $\mathbf{D}$-vector is orthogonal to the displacement vector between neighbouring sites. (c) In bulk materials with horizontal breaking of the inversion symmetry induced by the crystal lattice, the $\mathbf{D}$-vector is collinear to the displacement vector between neighbouring sites.

combining a thin ferromagnetic film (e.g., Fe, Co) with a non-magnetic heavy metal substrate exhibiting strong spin-orbit coupling ( $\mathrm{Pt}, \mathrm{Ir}$, etc). The orientation of the Dvector depends on the symmetries of the system. For a thin film lying in the $x y$-plane, such that the vertical inversion symmetry is broken along the $z$-axis, as is the case in Fig. 2.2a, the $\mathbf{D}$-vector is orthogonal to the displacement vector between atomic sites, and is expressed as $\mathbf{D}_{i j}=D \mathbf{e}_{z} \times \mathbf{r}_{i j}$, where $\mathbf{r}_{i j}=\mathbf{r}_{j}-\mathbf{r}_{i}$ is the in-plane displacement vector between sites $i$ and $j$ [89] (Fig. 2.2b). On the other hand, in bulk materials where the horizontal inversion symmetry is broken by the crystal lattice, the $\mathbf{D}$-vector is aligned with the displacement vector between atomic sites, and is expressed as $\mathbf{D}_{i j}=D \mathbf{r}_{i j}$ (Fig. 2.2c). 
In the continuum approximation, the DMI is described by combinations of Lifshitz invariants [89],

$$
L_{i j}^{(k)}=m_{i} \frac{\partial m_{j}}{\partial x_{k}}-m_{j} \frac{\partial m_{i}}{\partial x_{k}},
$$

where $i, j, k$ denote Cartesian indices. The interfacial form of the DMI with symmetry breaking along $z$ can be written as

$$
\mathscr{E}_{\mathrm{DM}}=\int \mathrm{d} V \mathscr{D}\left(L_{z x}^{(x)}+L_{z y}^{(y)}\right)=\int \mathrm{d} V \mathscr{D}\left(\left[m_{z} \frac{\partial m_{x}}{\partial x}-m_{x} \frac{\partial m_{z}}{\partial x}\right]+i d .(x \rightarrow y)\right) .
$$

$\mathscr{D}$ is the continuum form of the DMI coupling constant expressed in units of energy per unit area $\left(\mathrm{J} / \mathrm{m}^{2}\right)$, with $\mathscr{D}=2 D / a^{2}$ on the simple cubic lattice.

\subsubsection{Zeeman Energy}

The Zeeman energy contribution comes from the interaction of the system with an external magnetic field $\mathbf{B}=\mu_{0} \mathbf{H}$, where $\mu_{0}$ is the magnetic permeability of vacuum. It is expressed as

$$
E_{Z}=-M_{s} \sum_{i=1}^{N} \mathbf{m}_{i} \cdot \mathbf{B}
$$

This term is minimized by aligning the magnetic moments along the external field. The continuous form of Eq. (2.9) is simply

$$
\mathscr{E}_{Z}=-\mathscr{M}_{s} \int \mathrm{d} V \mathbf{m} \cdot \mathbf{B}
$$

\subsubsection{Magnetic anisotropy}

\section{Magnetocrystalline anisotropy}

In magnetic crystals, some crystallographic axes are more favourable than others for the magnetization to align along. This effect is known as the magnetocrystalline anisotropy. It originates from the interplay between spin-orbit coupling and magnetostatic interactions in the following way: the spins interact with the orbital motion by means of spin-orbit coupling, and the orbital motion in turns interacts with the crystal structure by means of the electrostatic fields and overlapping wavefunctions of neighbouring atoms [90]. We focus on systems in which the main magnetocrystalline anisotropy contribution gives rise to an easy axis, so that the associated energy takes on the form,

$$
E_{\mathrm{an}}=-K_{u} \sum_{i=1}^{N}\left(\mathbf{m}_{i} \cdot \mathbf{e}_{\mathrm{an}}\right)^{2}
$$


where $K_{u}>0$ is the anisotropy constant, and $\mathbf{e}_{\mathrm{an}}$ is known as the easy axis. In the continuum limit,

$$
\mathscr{E}_{\mathrm{an}}=-\mathscr{K}_{u} \int d V\left(\mathbf{m} \cdot \mathbf{e}_{\mathrm{an}}\right)^{2},
$$

with $\mathscr{K}_{u}=K_{u} / a^{3}$ expressed in $\mathrm{J} / \mathrm{m}^{3}$ on the simple cubic lattice. In magnetic thin films lying in the $x y$-plane with a perpendicular easy axis such that $\mathbf{e}_{\mathrm{an}}=\mathbf{e}_{z}$, the interaction energy of $\mathbf{m}_{i}$ with the easy axis can simply be expressed as $-K_{u} m_{z, i}^{2}$.

\section{Magnetostatic contribution}

Another contribution to the total anisotropy comes from dipole-dipole couplings between magnetic moments. Each magnetic moment constitutes a magnetic dipole and therefore contributes to the total dipolar field of the sample. Formally, the interaction potential that arises from the dipole-dipole coupling between two magnetic moments $\mathbf{m}_{i}$ and $\mathbf{m}_{j}$ takes the form

$$
E_{i j, \mathrm{dip}}=\frac{I_{\mathrm{dip}}}{\left|\mathbf{r}_{i j}\right|^{3}}\left(\mathbf{m}_{i} \cdot \mathbf{m}_{j}-3\left(\mathbf{m}_{i} \cdot \hat{\mathbf{r}}_{i j}\right)\left(\mathbf{m}_{j} \cdot \hat{\mathbf{r}}_{i j}\right)\right)
$$

where $\mathbf{r}_{i j}=\mathbf{r}_{j}-\mathbf{r}_{i}, \hat{\mathbf{r}}_{i j}=\mathbf{r}_{i j}|| \mathbf{r}_{i j} \mid$, and $I_{\text {dip }}$ is the strength of the dipolar coupling. The combined effect of the $N-1$ other magnetic moments on $\mathbf{m}_{i}$ constitutes the local dipolar field $\mathbf{H}_{d}^{i}=-\mu_{0}^{-1} \partial E_{\mathrm{dip}} / \partial \mathbf{M}_{i}$. In the continuum limit, the coupling of the magnetization with the dipolar - or demagnetizing - field $\mathbf{H}_{d}$ generated by the material constitutes the magnetostatic contribution to the energy. Combining $\mathbf{B}=\mu_{0}(\mathbf{H}+\mathscr{M})$ and Maxwell's equation, $\nabla \cdot \mathbf{B}=0$, it follows that $\mathbf{H}_{d}$ is the field that arises from the divergence of the magnetization:

$$
\nabla \cdot \mathbf{H}_{d}=-\nabla \cdot \mathscr{M}
$$

In ferromagnets, the interplay between magnetostatic energy and isotropic exchange is responsible for the emergence of uniformly magnetized domains with different orientations. This translates a tendency towards demagnetization, and is the reason why bulk magnets which have not been subjected to a magnetic field are usually found in a state of zero magnetization. $\mathbf{H}_{d}$ couples to the magnetization, $\mathscr{M}$, like an external field, in the form $-\frac{\mu_{0}}{2} \mathbf{H}_{d} \cdot \mathscr{M}$. In uniformly magnetized ferromagnets, the demagnetizing field may be expressed in terms of the demagnetizing tensor $\mathbf{N}$ as $\mathbf{H}_{d}=\mathbf{N} \cdot \mathscr{M}$.

In thin magnetic films, the pseudo magnetic charges on the surface are minimized if the magnetization lies in the plane of the film, so that the demagnetizing field can be approximated in the form $\mathbf{H}_{d}=-\mathscr{M}_{z} \mathbf{e}_{z}$ [91]. In the zero-thickness limit of ultrathin films, where dipolar couplings become local, this can be thought of as shape anisotropy, 
and the effective anisotropy constant becomes $\mathscr{K}_{\text {eff }}=\mathscr{K}_{u}-\mu_{0} \mathscr{M}_{s}^{2} / 2$ [92]. $\mathscr{K}_{\text {eff }}>0$ defines an easy-axis and corresponds to perpendicularly magnetized thin films, while $\mathscr{K}_{\text {eff }}<$ 0 corresponds to an easy-plane configuration. In the rest of this work, we focus on the former case, and we write $\mathscr{K}_{\text {eff }}=\mathscr{K}$, and $K=\mathscr{K} a^{3}$, to simplify the notation. In the end, in its atomistic form, the sum of the magnetocrystalline and magnetostatic contributions to the anisotropy in a thin magnetic film with perpendicular easy axis reduces to:

$$
E_{\mathrm{an}}=-K \sum_{i=1}^{N} m_{z, i}^{2}
$$

and

$$
\mathscr{E}_{\text {an }}=-\mathscr{K} \int d V m_{z}^{2}
$$

\subsubsection{Summary of atomistic and micromagnetics quantities}

In Table 2.1, we give a summary of the different physical quantities defined in this section with their corresponding symbol, unit, and the relations between the atomistic and micromagnetics quantities. We also define the following reduced dimensionless atomistic quantities that we shall use throughout this work: $j_{\alpha}=J_{\alpha} / J_{1}$ in which $(\alpha=$ $1,2,3) ; d=\left|\mathbf{D}_{i j}\right| / J_{1} ; k=K / J_{1} ; b=B M_{s} / J_{1}$. In the previous relations, $J_{\alpha}$ corresponds to the the isotropic exchange coupling constant between $\alpha$ th nearest neighbours, with $J_{\text {ex }}=J_{1}$ for first nearest neighbours. The atomistic exchange coupling constants, $J_{\alpha}$ and $D$, are given per site so that, for instance, the total isotropic exchange energy between two parallel spins is $-2 J_{\mathrm{ex}}$.

\subsection{Ferromagnetic domain walls}

The state of a ferromagnetic crystal which has not been subjected to an external magnetic field is usually that of zero magnetization. This is explained by the fact that crystals are divided into a finite number of regions of uniform magnetization, known as magnetic domains, such that, in the demagnetized state, the resulting magnetization of the crystal is zero. The domain boundaries, or domain walls, are regions usually much smaller than the domains themselves, in which the magnetization gradually rotates from one orientation to the other (Figs. 2.3a and 2.3b). They pertain to a class of solutions known as (static) topological solitons, i.e., solitonic solutions that are statically stable due to topological constraints. The formation of domains lowers the magnetostatic energy of the sample by reducing the magnetic charges on the surface, but raises the exchange energy by creating regions where the spins are not collinear. For that reason, domains are favourable if the size of the system is big enough as to ensure that 


\begin{tabular}{|c|c|c|c|c|c|c|}
\hline \multirow[t]{2}{*}{ Quantity } & \multicolumn{3}{|c|}{ Atomistic (simple cubic) } & \multicolumn{2}{|c|}{ Micromagnetics } & \multirow[t]{2}{*}{ Relation } \\
\hline & Symbol & Unit & Reduced & Symbol & Unit & \\
\hline Lattice constant & $a$ & $\mathrm{~m}$ & - & \multicolumn{2}{|c|}{-} & - \\
\hline $\begin{array}{c}\text { Saturation } \\
\text { magnetization }\end{array}$ & $M_{S}$ & A. $m^{2}$ & - & $\mathscr{M}_{s}$ & $\mathrm{~A} / \mathrm{m}$ & $\begin{array}{c}\mathscr{M}_{s}= \\
M_{s} / a^{3}\end{array}$ \\
\hline $\begin{array}{l}\text { Isotropic exchange } \\
\text { strength }\end{array}$ & $J_{\mathrm{ex}}=J_{1}$ & $\mathrm{~J}$ & 1 & A & $\mathrm{J} / \mathrm{m}$ & $A=J_{1} / a$ \\
\hline DMI strength & $D$ & $\mathrm{~J}$ & $\begin{array}{c}d= \\
D / J_{1}\end{array}$ & $\mathscr{D}$ & $\mathrm{J} / \mathrm{m}^{2}$ & $\begin{array}{c}\mathscr{D}= \\
2 D / a^{2}\end{array}$ \\
\hline Anisotropy constant & $K$ & $\mathrm{~J}$ & $k=K / J_{1}$ & $\mathscr{K}$ & $\mathrm{J} / \mathrm{m}^{3}$ & $\begin{array}{l}\mathscr{K}= \\
K / a^{3}\end{array}$ \\
\hline $\begin{array}{l}\text { Magnetic field } \\
\text { (induction) }\end{array}$ & $B$ & $\mathrm{~T}$ & $\begin{array}{c}b= \\
B M_{S} / J_{1}\end{array}$ & idem a & mistic & - \\
\hline $\begin{array}{c}\text { Magnetic } \\
\text { permeability of } \\
\text { vacuum }\end{array}$ & $\mu_{0}$ & $\mathrm{~N} / \mathrm{A}^{2}$ & - & idem a & nistic & - \\
\hline
\end{tabular}

Table 2.1: Symbols, corresponding units and relation between atomistic and micromagnetics quantities. The atomistic exchange couplings are given per site.

(a)

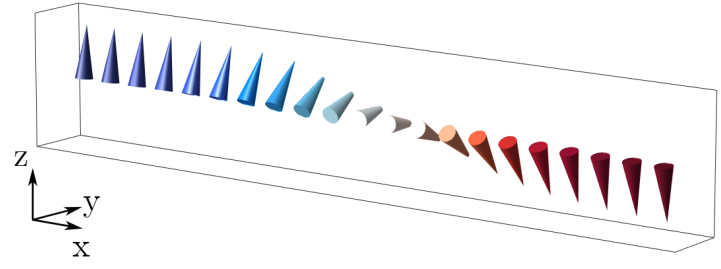

(b)
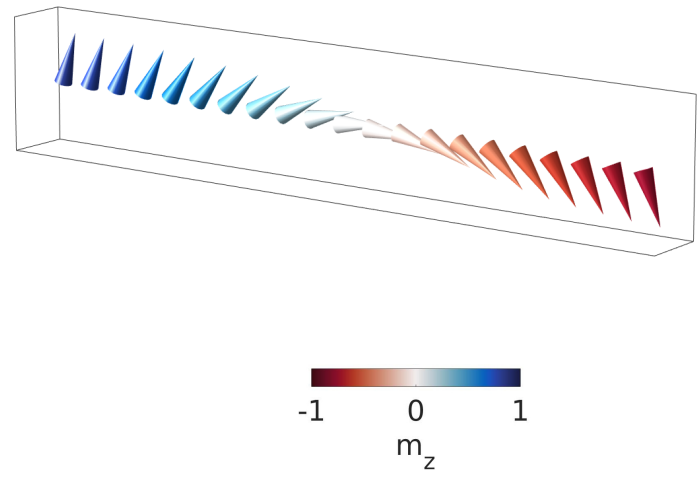

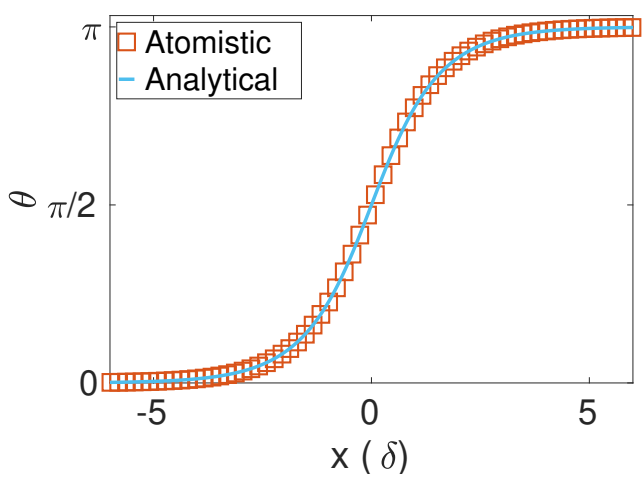

(c)

Figure 2.3: (a) Bloch $\pi$-wall in which the magnetization rotates in the plane of the wall, (b) Néel $\pi$-wall in which the magnetization rotates perpendicular to the plane of the wall, (c) analytical micromagnetic and numerical atomistic $\pi$-domain wall profiles as a function of the spatial displacement from the wall centre expressed in units of the characteristic wall width, $\delta$ (Eq. (2.31)). The micromagnetic analytical expression of $\theta(x)$ is given in Eq. (2.32), where $\theta$ is the angle of the magnetization with the easy-axis. Here, it corresponds to the polar angle with the $z$-axis, as defined in Fig. $2.1 \mathrm{~b}$ and Eq. (2.3). 
the reduction in magnetostatic energy is higher than the gain in exchange energy.

\subsubsection{Characteristic wall profile}

The width of a domain wall results from the competition between anisotropy and isotropic exchange energies. The more gradual the rotation of the magnetization, the lower the exchange energy. On the other hand, the anisotropy favours particular orientations of the magnetization along the easy axes and/or easy planes of the material. In what follows, we derive the characteristic quantities in a one-dimensional wall - i.e., wall profile, wall width, and wall energy. The derivation is based on that of Ref. [83].

We consider a one-dimensional chain of magnetic moments along the $x$-axis, with an easy-axis along the $z$ direction. We look at a $\pi$-wall separating two domains in which the magnetization goes from $+z$ to $-z$, as shown in Fig. 2.3. $\theta=\cos ^{-1} m_{z}$ is the angle between $\mathbf{m}$ and the $z$-axis (Fig. 2.1b), and we assume that the rotation occurs in a great enough number of steps for $\theta(x)$ to be considered a continuous variable. In this context, the interactions can then be written in the continuum approximation. If $f(\theta)$ is the anisotropy energy per unit volume, the total energy density per unit area of the wall is given by the sum of the exchange energy contribution, $\sigma_{\text {ex }}$, and the anisotropy energy contribution, $\sigma_{\text {an }}$,

$$
\sigma=\sigma_{\mathrm{ex}}+\sigma_{\mathrm{an}}=\int_{-\infty}^{+\infty}\left[A\left(\frac{\partial \theta}{\partial x}\right)^{2}+f(\theta)\right] d x
$$

A small change, $\delta \theta$, in $\theta$ induces the following change in $\sigma$ :

$$
\delta \sigma=\int_{-\infty}^{+\infty}\left[2 A \frac{\partial \theta}{\partial x} \frac{\partial}{\partial x} \delta \theta+\frac{\partial f(\theta)}{\partial \theta} \delta \theta\right] d x
$$

We integrate the first term by parts:

$$
\int_{-\infty}^{+\infty} 2 A \frac{\partial \theta}{\partial x} \frac{\partial}{\partial x} \delta \theta=\left[2 A \frac{\partial \theta}{\partial x} \delta \theta\right]_{-\infty}^{+\infty}-\int_{-\infty}^{+\infty} 2 A \frac{\partial^{2} \theta}{\partial x^{2}} \delta \theta d y
$$

and notice that

$$
\left[2 A \frac{\partial \theta}{\partial x} \delta \theta\right]_{-\infty}^{+\infty}=0
$$

since $\theta(x)$ is assumed constant inside the domains. The condition for the wall to be stable is obtained by minimizing the energy: $\delta \sigma=0$, that is to say,

$$
\delta \sigma=\int_{-\infty}^{+\infty}\left[\frac{\partial f(\theta)}{\partial \theta}-2 A \frac{\partial^{2} \theta}{\partial x^{2}}\right] \delta \theta d x=0
$$


Eq. (2.21) is true for any arbitrary $\delta \theta$ if

$$
\frac{\partial f(\theta)}{\partial \theta}-2 A \frac{\partial^{2} \theta}{\partial x^{2}}=0
$$

which is the Euler equation of the one-dimensional variational problem. Multiplying Eq. (2.22) by $\partial \theta / \partial x$ and integrating, we obtain an expression for the anisotropy energy:

$$
\begin{gathered}
\int \frac{\partial f(\theta)}{\partial \theta} \frac{\partial \theta}{\partial x} d x=2 A \int \frac{\partial^{2} \theta}{\partial x^{2}} \frac{\partial \theta}{\partial x} d x \\
f(\theta)=A\left(\frac{\partial \theta}{\partial x}\right)^{2},
\end{gathered}
$$

which is the same as the exchange energy, and from Eq. (2.24),

$$
d x=\sqrt{A} \frac{d \theta}{\sqrt{f(\theta)}}
$$

The energy per unit area of the wall is therefore

$$
\begin{aligned}
\sigma & =2 \int f(\theta) d x \\
& =2 \int f(\theta) \frac{\partial x}{\partial \theta} d \theta, \\
& =2 \sqrt{A} \int \sqrt{f(\theta)} d \theta,
\end{aligned}
$$

where we have used Eq. (2.25) in the last step. With an uniaxial anisotropy, $f(\theta)=$ $\mathscr{K} \sin ^{2} \theta$, and so the energy of a $\pi$-wall is

$$
\sigma_{0}=2 \sqrt{A \mathscr{K}} \int_{0}^{\pi} \sin \theta d \theta=4 \sqrt{\mathscr{K} A} .
$$

We note that with the above choice of $f(\theta)$, Eq. (2.22) becomes

$$
\sin \theta-\frac{A}{\mathscr{K}} \frac{\partial^{2} \theta}{\partial x^{2}}=0,
$$

which is the static sine-Gordon equation (Eq. (1.1)). By choosing the origin at the centre of the wall, it follows from Eq. (2.25) that the wall profile is expressed as

$$
x(\theta)=\sqrt{\frac{A}{\mathscr{K}}} \int_{\pi / 2}^{\theta} \frac{d \theta^{\prime}}{\sin \theta^{\prime}}=\sqrt{\frac{A}{\mathscr{K}}} \ln \left(\tan \frac{\theta}{2}\right) .
$$


The characteristic (asymptotic) width of the wall $\delta$ emerges from Eq. (2.29),

$$
\exp \left(\frac{x}{\sqrt{A / \mathscr{K}}}\right)=\exp \left(\frac{x}{\delta}\right)=\tan \frac{\theta}{2},
$$

where

$$
\delta=\sqrt{A / \mathscr{K}}
$$

and

$$
\theta(x)=2 \arctan \left(e^{x / \delta}\right),
$$

which we plot as a continuous blue line in Fig. 2.3c.

We distinguish between Bloch walls [93], in which the magnetization rotates in the plane of the wall (Fig. 2.3a), and Néel walls, in which the magnetization rotates perpendicular to the wall plane (Fig. 2.3b). Depending on the sample's shape and the magnetization direction inside domains, the type of wall that minimizes the magnetostatic energy is favoured against the other. In thin films magnetized out-of-plane, and in the absence of DMI, Bloch walls are more favourable as they minimize volume charges i.e., the magnetic charge density $\rho_{m}=-\nabla \cdot \mathbf{m}$ reduces to $\rho_{m}=-d m_{x} / d x=0$ in the Bloch wall, where $x$ is the propagation direction. This result, however, becomes less true in ultrathin films, since the energy difference between Bloch and Néel walls decreases with the film thickness [94]. Additionally, in the absence of chiral couplings, clockwise and counterclockwise directions of the magnetization rotation inside the wall are degenerate.

\subsubsection{Effect of chiral couplings}

In thin films with perpendicular anisotropy, the competition between the DMI and magnetostatic energy results in a progressive transition from Bloch walls at $\mathscr{D}=0$, to Néel walls with increasing $\mathscr{D}$. The DMI also selects a preferred handedness, or chirality, of the wall. The wall energy in the presence of DMI becomes $\sigma=\sigma_{0} \pm \pi \mathscr{D}[95,96]$. When the chirality of the wall is the one favoured by the DMI, $\sigma=\sigma_{0}-\pi \mathscr{D}$ and the wall is more energetically favourable than its counterpart of opposite chirality. Domain walls in PMA materials under uncompensated DMI have been named Dzyaloshinskii walls [96]. Above a certain critical value $\mathscr{D}_{c}$ of the DMI, expressed as $\mathscr{D}_{c}=4 \mathscr{K} \delta / \pi=\sigma_{0} / \pi$, the spin spiral state replaces the collinear state as the ground state of the system. 


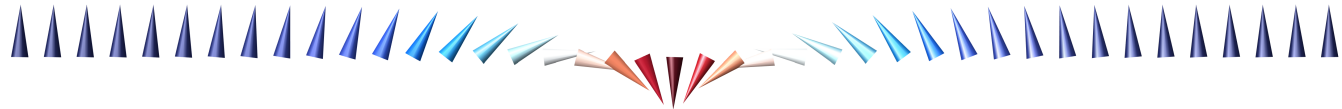

(a)

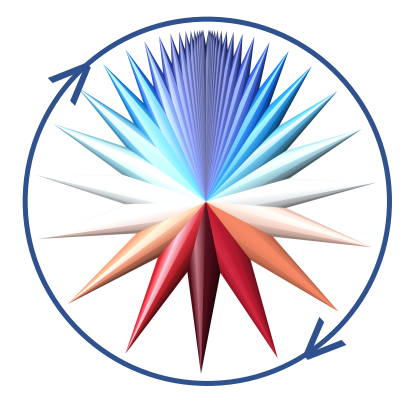

(b)

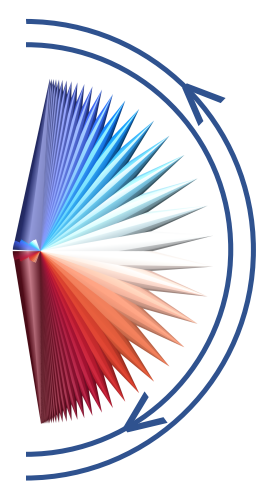

(c)

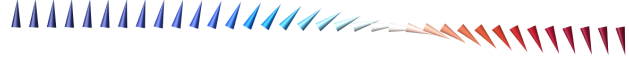

-..

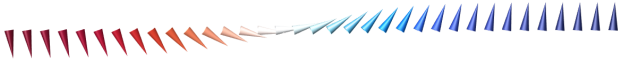

(d)

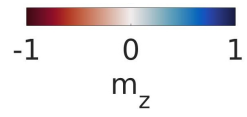

Figure 2.4: $(\mathrm{a}, \mathrm{b})$ Pair of Néel $\pi$-walls under a perpendicular applied field with fixed sense of rotation where (b) shows the stereographic projection onto the unit circle, $S^{1}$. The magnetization rotates in two-dimensional space from $\theta=0$, to $\pi$, then from $\pi$ to $2 \pi$, which results in a winding number $n=1$, and thus a topologically nontrivial spin texture with respect to the easy plane (soliton-soliton pair). In this case, the domain of down-magnetization in the middle can be reduced in size by a magnetic field applied along $+z$. (c, d) Pair of Néel $\pi$-walls at zero applied field with two different senses of rotation where (c) shows the stereographic projection onto the unit circle, $S^{1}$. The magnetization rotates in two-dimensional space from $\theta=0$, to $\pi$, then from $\pi$ to 0 , which yields a vanishing winding number, $n=0$, associated with a topologically trivial state (soliton-antisoliton pair). In this case, applying a magnetic field along $+z$ tends to destroy the walls and results in a single uniformly magnetized state along the field direction.

\subsubsection{Winding number and topology}

We now consider two $\pi$-walls, separating three magnetic domains. In the previously considered planar spin model, the spins are arranged along a one-dimensional chain and rotate strictly into the easy plane, such that $\theta(x)$ describes the magnetization. The order-parameter space is the unit circle, $S^{1}$. In this configuration where $\theta(x \rightarrow \pm \infty)=0$, the one-dimensional physical space, $\mathbb{R}^{1}$, is topologically equivalent to $S^{1}$, and we have the map $f: S^{1} \rightarrow S^{1}$, which may be classified by the homotopy class $\pi_{1}\left(S_{1}\right)=\mathbb{Z}$. Topologically distinct configurations are then defined by how many times the magnetization 
wraps the unit circle $S^{1}$. This is measured by the winding number - or degree of mapping $-\operatorname{deg} f=n$, defined as [7]

$$
n=\frac{1}{2 \pi} \int_{-\infty}^{+\infty} d x \partial_{x} \theta
$$

If the two $\pi$-walls exhibit the same sense of rotation - or chirality - as is the case in Fig. 2.4a, the magnetization rotates from $\theta=0$ to $\pi$, and then from $\pi$ to $2 \pi$, and therefore wraps around the unit circle one time. This can be visualized through the stereographic projection of the chiral wall shown in Fig. 2.4b. In this case, the winding number is $n= \pm 1$, and the structure is topologically nontrivial with respect to the easy plane. This configuration is referred to as soliton-soliton pair. In the continuum limit, it is topologically stable since, within the strict planar rotation model, there exists no continuous path in parameter-space that leads to the destruction of the wall. In particular, adding a DMI contribution to the present structure results in the selection of a specific sense of rotation - or chirality - which in turn favours topologically nontrivial pair of walls. On the other hand, in the absence of chiral couplings and if both $\pi$-walls possess an opposite sense of rotation, $\theta$ goes from 0 to $\pi$, and back to 0 , which in turn yields $n=0$. This configuration is shown in Fig. 2.4d, and Fig. 2.4c shows the corresponding stereographic projection. The achiral pair of walls is topologically trivial, as it is topologically equivalent to the collinear state, and is not robust against deformations. It corresponds to a soliton-antisoliton pair. Nevertheless, within an atomistic description, the chiral pair of walls can also be destroyed by strong enough external effects. Additionally, we can note that in real systems, anisotropies are finite, and so the strict planar model does not hold. The order parameter space is, in fact, the 2-sphere $S^{2}$, and the pair of chiral walls may be destroyed if the spins rotate along $y$ (case of "escape via the third dimension" [97]). Indeed, in a one-dimensional chain, topologically nontrivial solutions do not exist, since $\pi_{1}\left(S^{2}\right)=0$.

While the notion of topological protection applies to the continuum limit, it cannot be exactly transposed to a discretized description of space. In real systems where magnetic moments are localized at atomic sites, energy barriers are finite, and so topological protection is not strict. Topologically nontrivial states can be destroyed if the energy barrier than separates them from the uniform state is overcome by external effects and/or thermal fluctuations. 


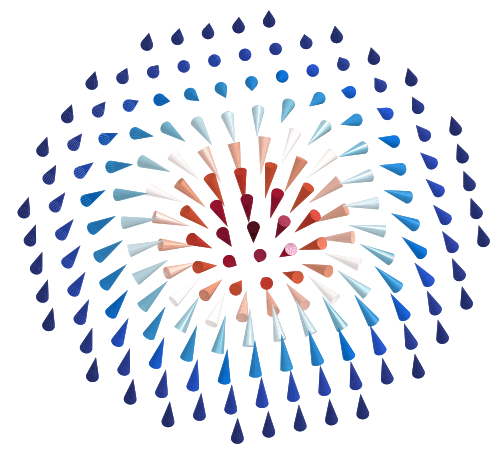

(a)

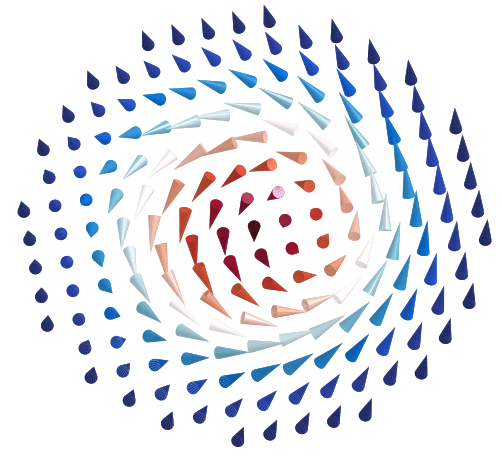

(c)

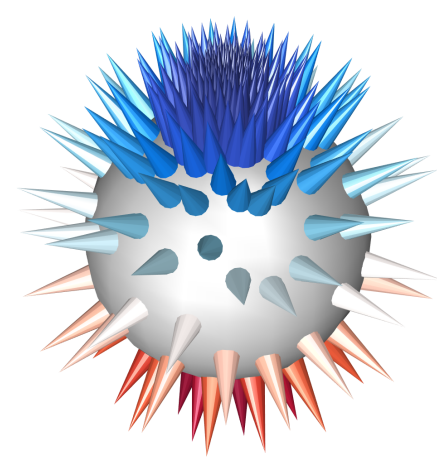

(b)

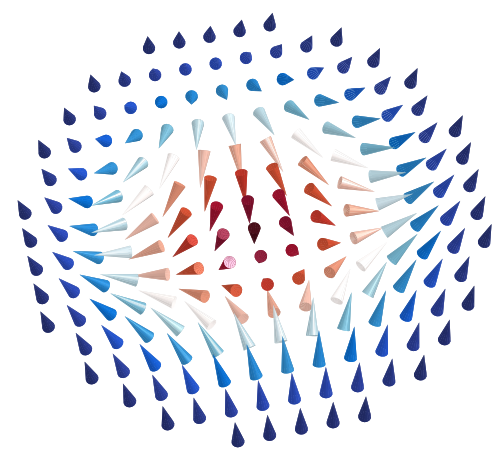

(d)

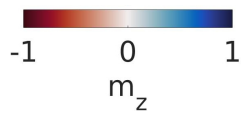

Figure 2.5: (a, b) A Néel "hedgehog" skyrmion with $\left(n, \phi_{0}\right)=(1, \pi)$ where (b) shows its stereographic projection onto the unit sphere, $S^{2}$. The magnetization along a radial cross-section resembles that of a chiral $2 \pi$ Néel wall. (c) A Bloch skyrmion with $\left(n, \phi_{0}\right)=(1, \pi / 2)$. The magnetization along a radial cross-section resembles that of a chiral $2 \pi$ Bloch wall. (d) An antiskyrmion with $\left(n, \phi_{0}\right)=(-1, \pi)$. The magnetization along a radial cross-section alternates between Bloch-like and Néel-like, depending on the value of the spatial polar angle $\varphi$ (see also Fig. 2.6).

\subsection{Magnetic skyrmions}

\subsubsection{General characteristics}

Magnetic skyrmions are topologically nontrivial, particle-like, solitonic magnetic textures which are exponentially localized in space. They typically exist in two dimensions, but can extend to three dimensions as skyrmion strings. Fig. 2.5 shows spin maps of some typical skyrmion configurations which we will describe later in this section. Originally, skyrmions were solitonic solutions derived by Skyrme [8] within his model for low energy dynamics between mesons and baryons. Skyrmions from the initial Skyrme model live on the three-dimensional space, and the term "baby skyrmions" was later introduced as solutions of the two-dimensional so-called "baby Skyrme" model [98,99]. In that sense, magnetic skyrmions are similar to baby skyr- 
mions. Within a skyrmion, the spins smoothly rotate in three dimensional space and their norm remains fixed, such that the order-parameter space is now the unit sphere, $S^{2}$. For an isolated skyrmion configuration, we assume that the magnetization tends asymptotically to a uniform value at infinity, i.e., $\mathbf{m}(\rho \rightarrow \infty)=$ cst. The physical space $\mathbb{R}^{2}$ may then be stereographically projected onto the punctured unit sphere $S^{2}$ with the missing point corresponding to $\rho \rightarrow \infty$, and we have the map $f: S^{2} \rightarrow S^{2}$, which may be classified by the homotopy class $\pi_{2}\left(S^{2}\right)=\mathbb{Z}$. [7, 100]. In Fig. 2.5b, we show the stereographic projection of the Néel skyrmion, as shown in Fig. 2.5a, onto $S^{2}$. We define the skyrmion number, or topological charge [101],

$$
N_{s}=\int \mathrm{d} \mathbf{r}^{2} \rho_{s}=\frac{1}{4 \pi} \int \mathrm{d} \mathbf{r}^{2} \mathbf{m} \cdot\left(\partial_{x} \mathbf{m} \times \partial_{y} \mathbf{m}\right),
$$

which corresponds to the degree of mapping, $N_{s}=\operatorname{deg} f$, and counts the number of times the spin configuration wraps the unit sphere, $S^{2}$. Skyrmions carry an integer topological charge, which implies that they are robust against small deformations and cannot be continuously unwound into the topologically trivial ferromagnetic state $\left(N_{s}=0\right) . \rho_{s}$, as defined by Eq. (2.34), is the local topological charge density.

We can use the polar coordinates $\rho=(\rho \cos \varphi, \rho \sin \varphi)$ to describe the spatial position, in which $\rho=\sqrt{x^{2}+x^{2}}$ is the in-plane distance from the skyrmion core, and $\varphi$ is the corresponding azimuth. The orientation of the spin vector $\mathbf{m}$ can be expressed in terms of spherical angles $(\theta, \phi)$ as given in Eq. (2.3). In the continuum limit, $\theta(\rho)$ only depends on $\rho$, and the in-plane skyrmion profile is

$$
\phi(\varphi)=n \varphi+\phi_{0}
$$

In the previous expression, $\phi_{0}$ is the helicity, and $n$ is the vorticity, or the $S^{1}$ - winding number along the domain wall delimiting the skyrmion, defined as [100]

$$
n=\frac{1}{2 \pi} \int_{0}^{2 \pi} d \varphi \partial_{\varphi} \phi
$$

Solutions that verify $n>0$ are called skyrmions, as shown in Figs. 2.5a and 2.5c, while solutions with $n<0$ are called antiskyrmions, as shown in Fig. 2.5d. In other words, when travelling around a circular contour at a fixed distance $\rho$ to the (anti)skyrmion core, the winding direction of the spins in a skyrmion matches the travelling direction along the contour. In an antiskyrmion, the spins rotate in a direction opposite to that of the travelling direction. From the symmetric ansatz $\theta(\rho)$ and $\phi(\varphi)$ (Eq. (2.35)), it follows that the skyrmion number in Eq. (2.34), which is the $S^{2}$-winding number, 
becomes

$$
N_{s}=\frac{n}{2}\left(m_{z}(0)-m_{z}(\infty)\right)
$$

Given the conditions $m_{z}(0)=-1$ and $m_{z}(\infty)=1$, we have $N_{s}=-n$. Amongst first order skyrmions $(n=1)$, we distinguish between Néel - or hedgehog - skyrmions with helicity $\phi_{0}=0$ or $\pi$, as shown in Fig. 2.5a, and Bloch skyrmions with $\phi_{0}=\pi / 2$ or $3 \pi / 2$ (Fig. 2.5c). Néel skyrmions exhibit a cycloidal magnetization texture along the radial direction, $\rho$, which is reminiscent of a chiral pair of Néel walls, such as the one discussed in Sec. 2.2.3 and shown in Fig. 2.4a, while Bloch skyrmions exhibit a helicoidal spin texture along the radial direction, which resembles that of a chiral pair of Bloch walls. In antiskyrmions $(n=-1)$, on the other hand, the spin texture along the radial direction alternates between a Néel-type and a Bloch-type pair of walls depending on the spatial angle $\varphi$. We show the detail of the spin texture of an antiskyrmion on the simple square lattice in Fig. 2.6, in which the radial directions corresponding to lattice axes $x$ and $y(\varphi=\{0, \pi / 2\})$ display clockwise- (CW) and counter-clockwise$(\mathrm{CCW})$ rotating Néel-type profiles, while along the lattice diagonals $(\varphi=\{\pi / 4,3 \pi / 4\})$, the magnetization is that of CW and CCW Bloch-type profiles. In higher-order skyrmion solutions $(|n|>1)$, in which the magnetization wraps the unit sphere more than once, the radial profile also alternates between Néel and Bloch. For instance, typical spin textures corresponding to a second-order skyrmion $(n=2)$, and a second-order antiskyrmion $(n=-2)$ are shown respectively in Figs. $2.9 \mathrm{~d}$ and $2.9 \mathrm{e}$.

Magnetic skyrmions are stabilized in ferromagnets by the introduction of a characteristic lengthscale via competing interactions. This is typically achieved in noncentrosymmetric magnets, in which chiral couplings arise in the form of DMI. That particular type of solution, commonly referred to as chiral skyrmions, were theoretically predicted and investigated in the 1990s [9-11]. In magnets with inversion symmetry, skyrmions can be stabilized via dipolar interactions (skyrmion bubbles, also referred to as bubble domains) [16-21], as well as frustrated exchange couplings [22-27]. In the rest of this section, we firstly define the topological charge on the discrete spin lattice. After that, we give details on the different categories of magnetic skyrmions according to their stabilization mechanism.

\subsubsection{Topological charge definition on the atomistic spin lattice}

In a continuum model, the topological charge is defined as its integral expression that we gave in Eq. (2.34), and corresponds to the number of times $\mathbf{m}(x, y)$ wraps the unit sphere $S^{2}$. On the discretized atomistic spin lattice, the topological charge needs to be redefined. A lattice of spins $\mathbf{m}\left(x_{i}, y_{i}\right)$ can be thought of as a net on $S^{2}$, with 


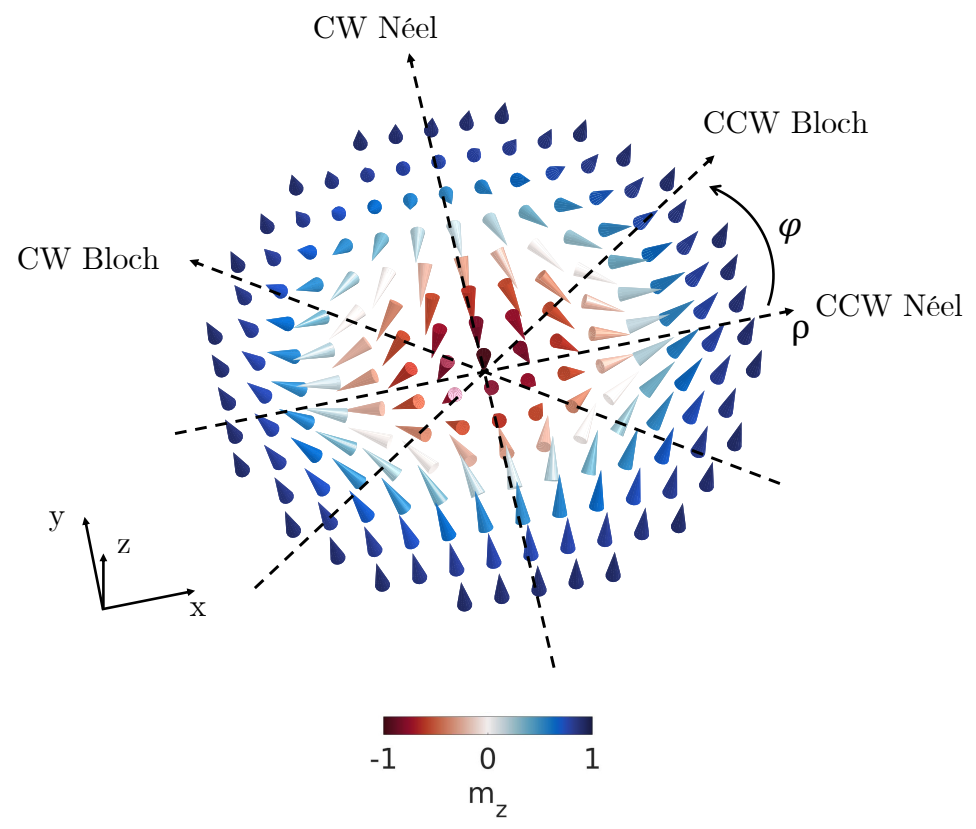

Figure 2.6: Anatomy of the antiskyrmion spin texture. The winding direction of the spins in the direction of increasing $\varphi$ is negative (i.e., clockwise $(\mathrm{CW})$ rotation of the magnetization for a counter-clockwise (CCW) rotation along the contour), yielding a negative winding number, $n=-1$. Depending on the value of $\varphi$, the spin texture along the radial direction $\rho$ alternates between Néel-type and Bloch-type with CW and CCW rotation of the spins.

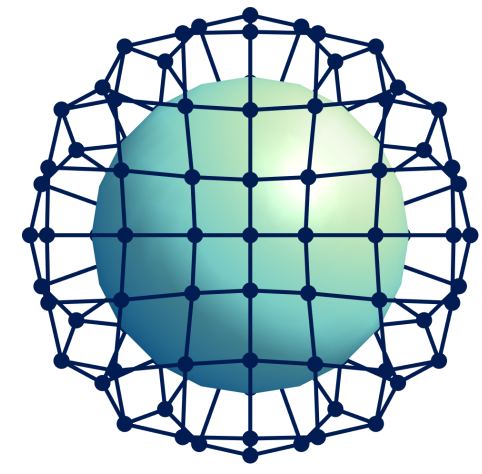

(a)
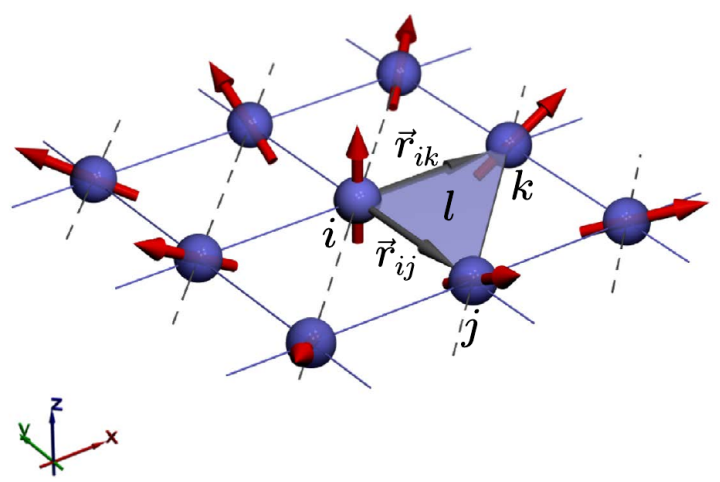

(b)

Figure 2.7: (a) Mapping of the spin configuration for an isolated skyrmion onto the unit sphere. Vertices correspond to the tip of the magnetic vectors with their origin in the centre of the sphere, and the edges represent the exchange coupling between first neighbours. The solid sphere inside the net represents the unit sphere. (b) Elementary triangles on the simple square lattice (image source: [102]). 
vertices $\mathbf{m}\left(x_{i}, y_{i}\right)=\mathbf{m}_{i}$, and rubber bands connecting $\mathbf{m}_{i}$ and $\mathbf{m}_{j}$ whenever sites $i$ and $j$ are nearest neighbours (Fig. 2.7a). In that case, the topological charge can be defined as the number of times the net winds around $S^{2}[103]$. Following the expression given in Ref. [104] whose derivation was based on Ref. [103], we give the topological charge on the discrete spin lattice,

$$
N_{s}=\frac{1}{4 \pi} \sum_{l} q_{l}
$$

with

$$
\tan \left(\frac{q_{l}}{2}\right)=\frac{\left[\mathbf{m}_{i}, \mathbf{m}_{j}, \mathbf{m}_{k}\right]}{1+\mathbf{m}_{i} \cdot \mathbf{m}_{j}+\mathbf{m}_{i} \cdot \mathbf{m}_{k}+\mathbf{m}_{j} \cdot \mathbf{m}_{k}} .
$$

The notation $\left[\mathbf{m}_{i}, \mathbf{m}_{j}, \mathbf{m}_{k}\right]=\mathbf{m}_{i} \cdot\left(\mathbf{m}_{j} \times \mathbf{m}_{k}\right)$ corresponds to the scalar triple product. The index $l$ runs over all the elementary triangles of the lattice, where $\mathbf{m}_{i}, \mathbf{m}_{j}, \mathbf{m}_{k}$ define the vertices of a triangle (Fig. 2.7b). The indices $i, j, k$ are attributed by rotating counterclockwise about the $z$-axis.

$$
Q_{l}=\frac{q_{l}}{4 \pi}
$$

is a discrete equivalent to the topological charge density $\rho_{s}$ (Eq. (2.34)) and may be interpreted as a local topological charge. The topological charge on the discrete spin lattice defined through Eqs. (2.38) and (2.39) should hold for all types of lattices.

In Fig. 2.7a, we show a "ball in a net" representation of the Néel skyrmion in Fig. 2.5a, which was also somewhat inspired by Fig. 3 in Ref. [80]. Vertices correspond to the tip of the magnetic vectors with their origin in the centre of the sphere, and the edges represent the exchange coupling between first neighbours. The solid sphere inside the net represents the unit sphere. The ferromagnetic background (spins along $+z)$ is found at the north pole of the sphere, while in this thesis, the core of a skyrmion typically points along the south pole (along $-z)$. The view is set just below the south pole, looking axially towards the $+z$ direction, so that the spin in the centre of the view corresponds to the core of the skyrmion. Annihilating a nontrivial topological texture to recover the uniformly magnetized state involves bringing the mesh back to the north pole by moving the vertices on the sphere. This can be thought of as taking a ball (solid sphere) out of a net (dark blue mesh) by deforming the rubber band net.

\subsubsection{Chiral skyrmions in non-centrosymmetric magnets}

The first theoretical prediction that the DMI can stabilize two-dimensional solitons dates back to 1989 [9]. Initially labelled "(chiral) magnetic vortices", they were later renamed "chiral magnetic skyrmions" to mark the distinction with vortices, in which the magnetization only spans over half of the unit sphere, yielding a half-integer topo- 


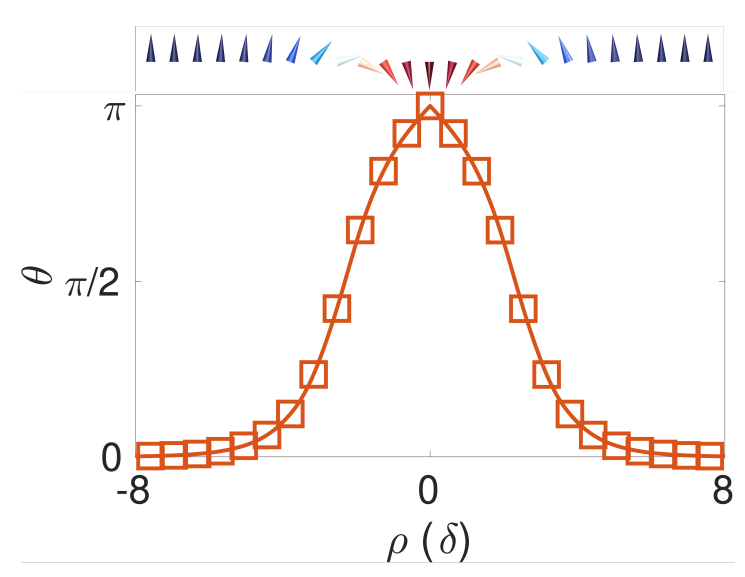

(a)

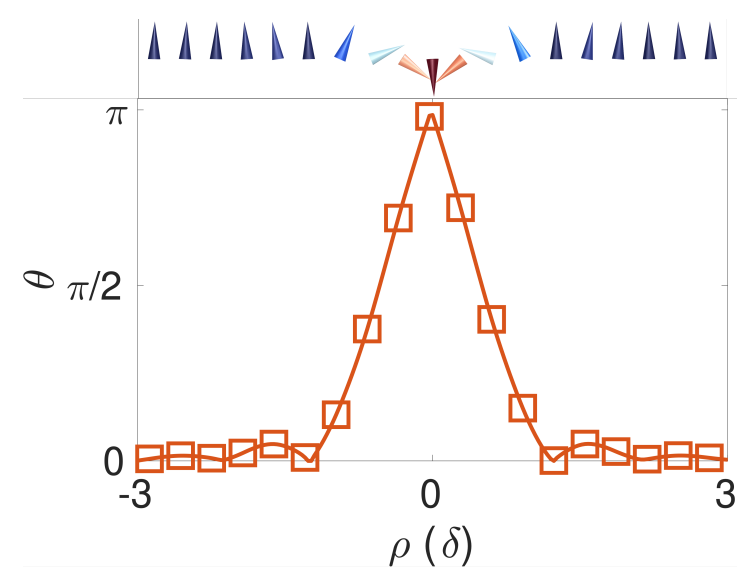

(b)

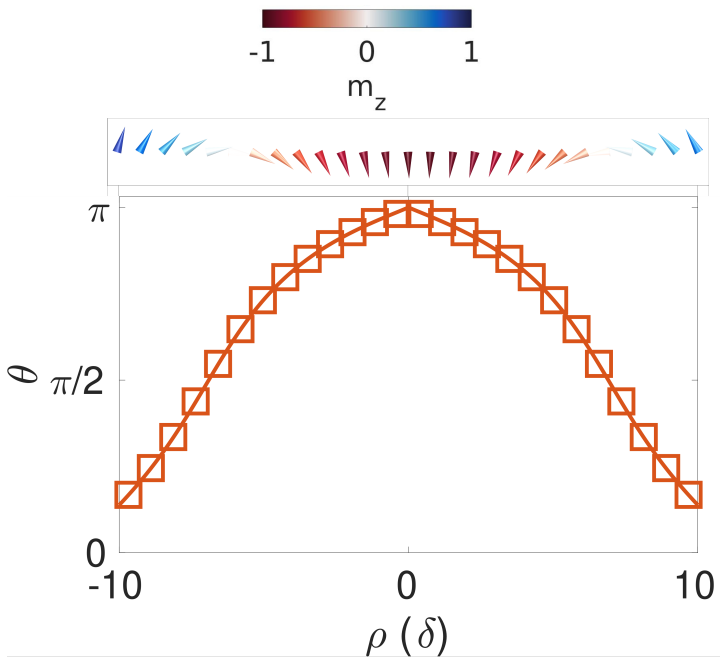

(c)

Figure 2.8: Numerical profile obtained from atomistic simulations (squares) and corresponding spline interpolation (line) for skyrmions solutions with different stabilization mechanisms, as a function of the radial spatial displacement from the skyrmion core, $\rho$, expressed in units of the characteristic domain wall width, $\delta$ (Eq. 2.31). $\theta$ is the polar angle with the easy axis, $z$. The spin maps at the top show the spin texture along a radial cross-section of the skyrmion. (a) Profile of a chiral Néel skyrmion stabilized by the DMI. The core is strongly localized, with linear variations of the polar angle, while the asymptotic profile of the tail far from the centre resembles that of a ferromagnetic domain wall. (b) Profile of a Néel skyrmion stabilized by frustrated exchange. The core is strongly localized. In the tail, the polar angle oscillates as a function of distance, indicating a reversal of helicity. (c) Profile of a Néel skyrmion bubble stabilized by dipolar couplings. The core is spatially extended and not strongly localized. 
logical charge (see Fig. 1.1b). A skyrmion lattice was first detected in the bulk of the B20 chiral magnet $\mathrm{MnSi}$ in reciprocal space, via neutron scattering [28]. Shortly after, Yu et al. reported the first real space observation of a skyrmion lattice in a thin film of $\mathrm{Fe}_{0.5} \mathrm{Co}_{0.5}$ by means of Lorentz transmission electron microscopy (TEM) [29]. These observations opened the way of potential spintronics applications of skyrmions in data storage and logic devices [38-42]. The existence of skyrmions was then confirmed in many other types of materials [30-33]. In particular, some well-studied transition-metal thin films and multilayers that give rise to a substantial interfacial DMI in which individual skyrmions were experimentally observed are $\mathrm{Ir} / \mathrm{Co} / \mathrm{Pt}$ [105], $\mathrm{Pt} / \mathrm{Co} / \mathrm{AlO}_{x}$ [106, 107], and $\mathrm{Pd} / \mathrm{Fe} / \mathrm{Ir}(111)$ [33, 43, 63, 108, 109]. In chiral magnets, in the absence of any other effect, the competition between isotropic exchange and DMI results in a ground state of spatially modulated spin spirals (SSs) with a fixed sense of rotation. Additional couplings such as anisotropies and external field can suppress the SS state in favour of the uniformly magnetized ferromagnetic (FM) state, from which isolated skyrmions can be stabilized as (meta)stable excitations. In the presence of perpendicular applied magnetic field, a thermodynamically stable skyrmion lattice phase appears at intermediate field values [11, 110].

The symmetries of the DMI favour a single class of solutions with fixed chirality and helicity. For instance, interfacial DMI favours Néel skyrmions (Fig. 2.5a), and bulk DMI favours Bloch skyrmions (Fig. 2.5c). Antiskyrmions (Figs. 2.5d and 2.6) can be stabilized by anisotropic DMI [111,112]. This has for instance been reported in tetragonal Heusler compounds, in which antiskyrmions were observed via Lorentz TEM [31]. Replacing $\mathbf{D}$ with $-\mathbf{D}$ results in skyrmion solutions of the opposite chirality. Nonetheless, the possibility to stabilize structures with an arbitrary integer topological charge in chiral magnets ("skyrmionic sacks") has recently been explored [113]. Far from the skyrmion core, the asymptotic behaviour of the spins along the radial direction $\rho$ is that of a ferromagnetic wall, $\theta(\rho) \sim \kappa e^{ \pm \rho / \delta}$, in which $\delta$ is the characteristic domain wall width (Eq. (2.31)) and $\kappa=\pi \mathscr{D} / 4 \sqrt{A \mathscr{K}}$, whereas the core is strongly localized, arrowlike shaped, with linear variation of the polar angle: $\theta(\rho) \sim \rho \mathscr{D} / A$ [114,115]. We show a typical chiral skyrmion profile along a radial cross-section in Fig. $2.8 \mathrm{a}$, where the spin texture is shown at the top. It follows that the characteristic size of chiral skyrmions is similar to that of ferromagnetic walls, while being spatially localized in all directions. Besides $\delta$, the other characteristic lengthscale in the system is $\zeta=2 A / \mathscr{D}$ [21, 107], which characterizes the period of the spatially modulated states that arise from the competition of isotropic exchange and DMI. There is no exact analytical expression for skyrmion profiles, however the double soliton ansatz of two overlapping $\pi$-walls at 
positions $\pm c$ works well $[97,108,116$ :

$$
\theta(\rho, c, \delta)=\sum_{+,-} \arcsin \left(\tanh \left(\frac{-\rho \pm c}{\delta}\right)\right),
$$

in which the sum is carried out over the two $\pi$-wall profiles with spatial displacement given respectively by $\rho+c$ and $\rho-c$.

\subsubsection{Skyrmions in frustrated magnets}

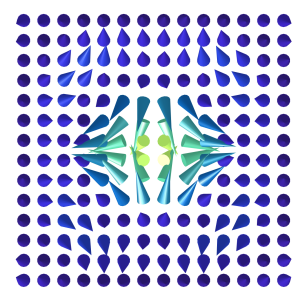

(a)

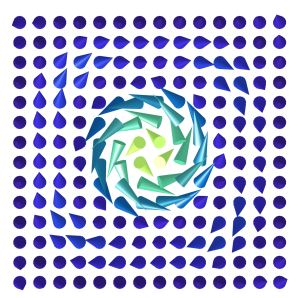

(b)

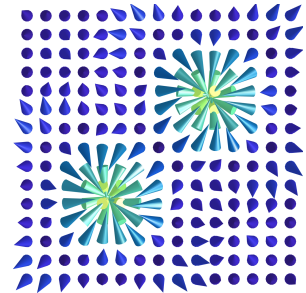

(f)

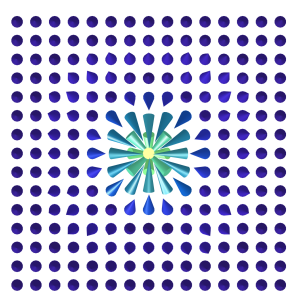

(c)

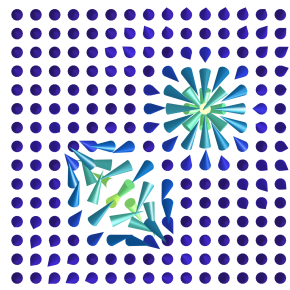

$(\mathrm{g})$

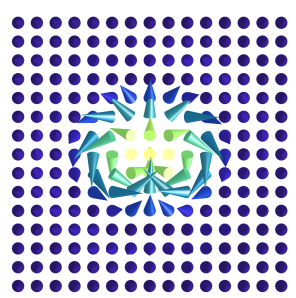

(d)

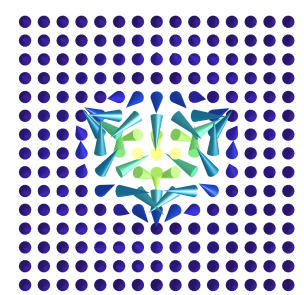

(e)

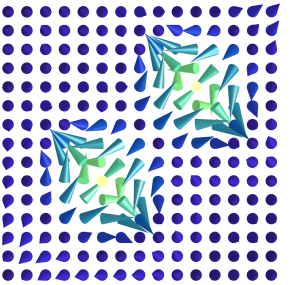

(h)

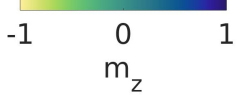

Figure 2.9: Different skyrmion solutions stabilized in a frustrated magnet with inversion symmetry: (a) antiskyrmion with $\left(n, \phi_{0}\right)=(-1,0)$, (b) skyrmion with $\left(n, \phi_{0}\right)=$ $(1, \pi / 2)$ (Bloch), (c) skyrmion with $\left(n, \phi_{0}\right)=(1, \pi)$ (Néel), (d) 2nd order skyrmion with $\left(n, \phi_{0}\right)=(2,3 \pi / 2)$, (e) 2nd order antiskyrmion with $\left(n, \phi_{0}\right)=(-2, \pi / 2)$, (f) skyrmion pair, (g) skyrmion/antiskyrmion pair, (h) antiskyrmion pair.

Frustrated isotropic exchange corresponds to the case where the coupling constant $J_{\alpha}$ changes sign depending on the shell of neighbours $\alpha=1,2,3 \ldots$, resulting in alternating ferromagnetic $\left(J_{\alpha}>0\right)$ and antiferromagnetic $\left(J_{\alpha}<0\right)$ couplings. For clarity, $\alpha=1$ corresponds to nearest neighbours, $\alpha=2$ to next-nearest neighbours, etc (see Fig. 2.1a). Exchange frustration has been shown to arise from interface effects in certain systems. $\mathrm{Pd} / \mathrm{Fe} / \mathrm{Ir}(111)$ is an experimentally well-studied system that was reported to host interface nanoscale skyrmions [33, 108]. Density functional theory (DFT) calculations have shown that for fcc stacking of the Pd layer, the isotropic exchange is ferromagnetic between first neighbours, but antiferromagnetic beyond first neighbours, leading to a 
SS ground state even without the inclusion of SOC (i.e., DMI) in the calculations [43]. More recently, a strong exchange frustration was reported in $\mathrm{Rh} / \mathrm{Fe} / \mathrm{Ir}(111)$, which was found to be a driving force behind the SS ground state [117]. Strong exchange frustration was also very recently revealed in $\mathrm{Rh} / \mathrm{Co} / \mathrm{Ir}(111)$ by spin-polarized scanning tunneling microscopy (SP-STM) measurements combined with DFT calculations [118]. In this system, skyrmions of approximately $5 \mathrm{~nm}$ in diameter were observed at $4 \mathrm{~K}$ at zero magnetic field in the virgin FM state. The exchange frustration was found to play an important role in their stabilization. For these reasons, studying skyrmion solutions stabilized by frustrated exchange is important for technological applications. In exchange-frustrated magnets, spatially modulated states can arise without the need for chiral couplings. For certain specific values of the exchange parameters, a spin-spiral ground state is realized in the absence of other interactions [22-27]. Similar to the case of chiral magnets, the SS state is suppressed by additional couplings such as external field and/or magnetic anisotropies, and the uniformly magnetized ferromagnetic state is recovered via a second-order phase transition [25]. From that state, metastable skyrmion solutions can be stabilized. In the presence of a perpendicular easy-axis anisotropy, the skyrmion lattice becomes thermodynamically stable at an intermediate applied field [25].

In comparison to chiral skyrmions stabilized by the DMI, where the type of the DMI determines the helicity and winding direction of the spins, exotic spin textures with various winding numbers and helicities can coexist in frustrated magnets, as seen in Fig. 2.9. In the absence of inversion symmetry breaking, skyrmions (Figs. 2.9b, 2.9c) and antiskyrmions (Fig. 2.9a) with opposite winding numbers are degenerate in energy. Additionally, the skyrmions possess a spin degree of freedom (where "spin" refers to the spin of the skyrmion as a particle), associated with a rotation of helicity. In Fig. $2.10 \mathrm{a}$, we show the profile of the mode corresponding to the rotation of helicity in a Néel skyrmion. In the absence of DMI, this mode is a mode of zero energy fluctuations, also known as a Goldstone mode. Goldstone modes and their implications are discussed later in Chapter 3, Bloch-type skyrmions (Fig. 2.9b), Néel-type skyrmions (Fig. 2.9c), and all intermediate helicity states are therefore, in the absence of DMI, degenerate in energy [25]. The skyrmion profile is also different from that of a chiral skyrmion. The polar angle $\theta(\rho)$ goes from $\theta(0)=\pi$ at the centre, to zero, and then oscillates around zero, indicating a reversal of helicity, as shown in Fig. 2.8b. As a consequence, the pairwise interaction potential of skyrmions is non-monotonic as a function of distance, and is found in turn positive (repulsive) and negative (binding) [22, 25], and depends on the relative helicities. It is therefore possible to stabilize a bound (anti)skyrmion/(anti)skyrmion pair (Figs. 2.9f, 2.9h, 2.9g). 


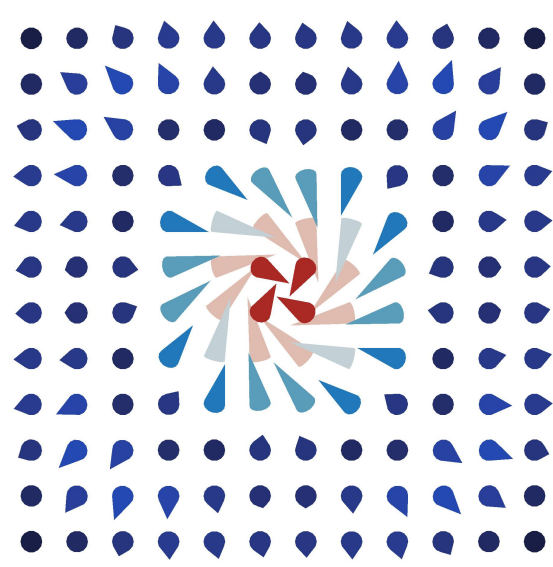

(a)

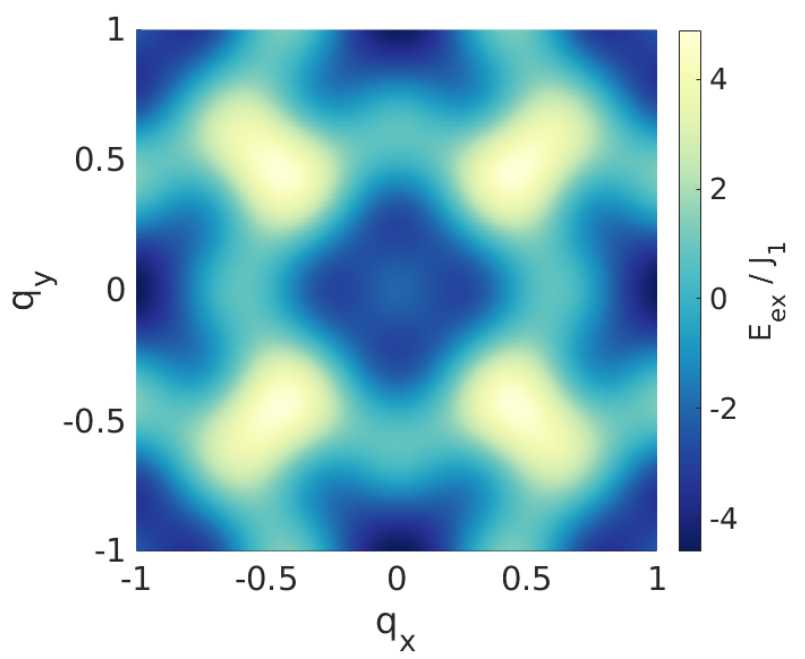

(b)

Figure 2.10: (a) Profile of the Goldstone mode associated with the rotation of helicity in the Néel skyrmion in the absence of inversion symmetry breaking $(d=0)$. At zeroDMI, Bloch and Néel skyrmions, as well as all skyrmion solutions with intermediate values of the helicity are degenerate in energy. (b) Isotropic exchange energy, $E_{\text {ex }}(\mathbf{q})$, in units of $J_{1}$ in the two-dimensional Brillouin zone of the simple square lattice for $\left(j_{2}, j_{3}\right)=(-0.35,-0.15)$. This particular choice of exchange coupling parameters gives rise to a minimum in the exchange energy around $q_{0 x} \approx q_{0 y} \approx 0.23$, which corresponds to a SS ground state.

We now focus on the frustrated $J_{1}-J_{2}-J_{3}$ magnet on the square lattice under a perpendicular applied field, and $(d, k)=(0,0)$. The upcoming derivation is based on that of Ref. [25]. The competing interactions can stabilize a state with non-zero ordering wave vector $\mathbf{q}$ as a ground state. At zero-temperature and below a critical applied field $B_{c}$, the ground state is a homogeneous spin spiral, and the orientation of spin $\mathbf{m}_{i}$ at site $\mathbf{r}_{i}$ is expressed as

$$
\mathbf{m}_{i}=\left(\sin \theta \cos \left(\mathbf{r}_{i} \cdot \mathbf{q}\right), \sin \theta \sin \left(\mathbf{r}_{i} \cdot \mathbf{q}\right), \cos \theta\right),
$$

where the canting angle $\theta$ is determined by $\cos \theta=B_{z} / B_{c}$. We express the wave vector of the two-dimensional Brillouin zone as

$$
\mathbf{q}=\frac{2 \pi}{a}\left(\begin{array}{l}
q_{x} \\
q_{y}
\end{array}\right)
$$

where $a$ is the lattice constant. Setting $\mathbf{m}_{i}=\mathbf{e}_{x}$ at position $\mathbf{r}_{i}=(0,0)$, its first nearest neighbours are located at

$$
\left\{\mathbf{r}_{j}\right\}=\{(a, 0),(-a, 0),(0, a),(0,-a)\}
$$


so that the total exchange energy between $\mathbf{m}_{i}$ and its first neighbours is

$$
E_{1}=-2 J_{1}\left(\cos \left(2 \pi q_{x}\right)+\cos \left(2 \pi q_{y}\right)\right)
$$

where we have chosen $\theta=\pi / 2$. Proceeding analogously for the second and third shells of neighbours, we obtain

$$
\begin{aligned}
E_{\mathrm{ex}}(\mathbf{q})= & -2 J_{1}\left(\cos \left(2 \pi q_{x}\right)+\cos \left(2 \pi q_{y}\right)\right) \\
& -2 J_{2}\left(\operatorname { c o s } \left(2 \sqrt{2} \pi\left(q_{x}+q_{y}\right)+\cos \left(2 \sqrt{2} \pi\left(q_{x}-q_{y}\right)\right)\right.\right. \\
& -2 J_{3}\left(\cos \left(4 \pi q_{x}\right)+\cos \left(4 \pi q_{y}\right)\right) .
\end{aligned}
$$

We then look for a set of $\left(j_{2}, j_{3}\right)$ for which there exists a non-zero wave vector $\mathbf{q}_{0}$ that minimizes $E_{\mathrm{ex}}\left(\mathbf{q}_{0}\right)$. In order to obtain skyrmions with sizes larger than the lattice parameter, we must limit the search to the large wavelength limit $q a \ll 1$, where $q=\sqrt{q_{x}^{2}+q_{y}^{2}}$. In Ref. [25], the exchange energy is expanded in small values of $q$. The spin lattice introduces a spatial anisotropy, which is minimized by tuning $\left(j_{2}, j_{3}\right)$. This yields the following reduced coupling parameters: $j_{2}=-0.35 ; j_{3}=-0.15$, with a minimum in the spin spiral energy around $q_{0 x} \approx q_{0 y} \approx 0.23$. Fig. 2.10b shows the value of $E_{\mathrm{ex}}(\mathbf{q})$ as given in Eq. (2.46) in the first Brillouin zone. The darker area around the center corresponds to the SS ground state. The value of $\mathbf{q}_{0}$ gives the period of the spin spirals and determines the equilibrium sizes of isolated skyrmion solutions.

\subsubsection{Skyrmion bubbles in dipolar magnets}

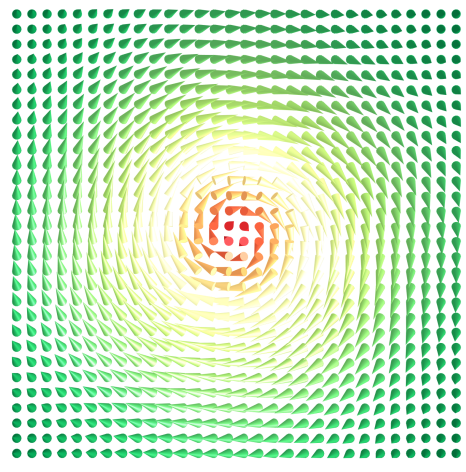

(a)

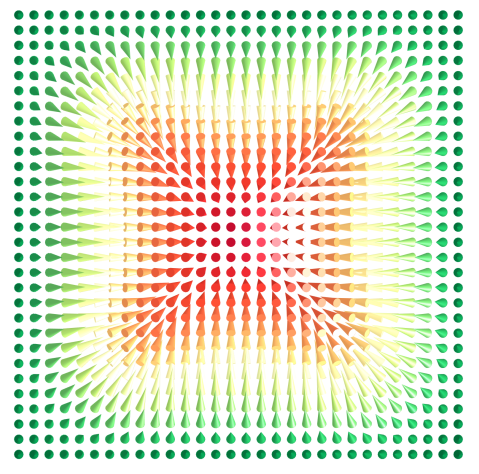

(b)

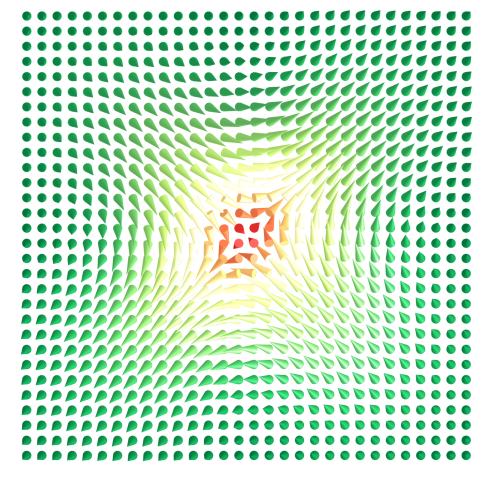

(c)

Figure 2.11: Examples of skyrmion bubbles: (a) Bloch-type bubble with $\left(n, \phi_{0}\right)=$ $(1,3 \pi / 2)$, (b) Néel-type bubble with $\left(n, \phi_{0}\right)=(1, \pi)$, (c) antiskyrmion bubble with $\left(n, \phi_{0}\right)=(-1, \pi / 2)$. 
Bubble domains, or skyrmion bubbles [91], originate from the tension of domain walls and surface depolarization as an effect of the sample's shape. In most cases, the profile consists in a spatially extended core with $\theta=\pi$, separated by a domain wall from the uniformly magnetized background where $\theta=0$. Contrary to chiral skyrmions and skyrmions stabilized by exchange frustration, the core does not exhibit strong localization, with a typically flat or quasi-flat $\theta(\rho)$ profile around the centre [114]. Although bubbles carry an integer topological charge, their typical sizes are found in the micrometer range and, unlike skyrmions, they usually cannot be reduced to nanoscale sizes. We show the spin maps of some bubble skyrmions in Fig. 2.11. In Fig. 2.8c, we plot the profile of the Néel bubble in Fig. $2.11 \mathrm{~b}$ along a radial cross-section. It shows that the core is much more spatially extended than that of the two skyrmions in Figs. $2.8 \mathrm{a}$ and $2.8 \mathrm{~b}$, with a smaller slope in the centre. Although it is not flat in this case, this seems to be due to the fact that the bubble is constrained inside the $50 \times 50$ simulated surface. The phase diagram in dipolar magnets is similar to that of chiral magnets, with the helical ground state at zero applied field, the bubble lattice at intermediate field and the uniformly magnetized state at higher field, from which metastable isolated bubbles may be stabilized [91,119]. Contrary to the DMI, the dipolar interactions do not select a single value of the helicity, so that solutions with $\phi_{0}=\pi / 2$ or $3 \pi / 2$ are degenerate (Fig. 2.11a), and so are solutions with $\phi_{0}=0$ or $\pi$ (Fig. 2.11b) [21]. Winding numbers of $n= \pm 1$ can also coexist (Fig. 2.11c).

\subsection{Summary}

In this chapter, we have seen that the main energy contributions in magnets are short-range exchange interactions and long-range dipolar interactions between magnetic moments, as well as interactions of the spins with the crystal lattice and external magnetic fields. The main contributions to the exchange interactions considered in this thesis are the isotropic Heisenberg exchange favouring collinear orientations of neighbouring spins, and the antisymmetric, anisotropic Dzyaloshinskii-Moriya interaction, which favours orthogonal orientations of neighbouring spins with a particular sense of rotation. The DMI arises from spin-orbit coupling in systems with broken inversion symmetry and is responsible for the emergence of noncollinear chiral magnetic orderings. DMI is found in the bulk of noncentrosymmetric crystals such as $\mathrm{MnSi}$, but can also be generated in small centrosymmetric magnetic structures such as thin films, by structural breaking of the inversion symmetry.

On our way to understanding magnetic skyrmions, we have looked at their onedimensional counterpart in the form of ferromagnetic walls in a chain of spins. Do- 
main walls are a form of topological solitons which arise as solutions to the static sineGordon equation in one dimension. In ferromagnets, they extend to higher dimensions between uniformly magnetized domains. Using a variational method, we derived the characteristic quantities in a domain wall, i.e., profile, width, and energy. We made the distinction between Bloch walls, in which the magnetization rotates in the plane of the wall, and Néel walls, in which the magnetization rotates perpendicular to the wall plane. We showed than a chiral pair of $\pi$-walls is topologically nontrivial with respect to the easy-plane, that is, if the spins in both walls rotate in the same direction, resulting in a soliton-soliton pair. Nevertheless, within a discretized description of space in which the spins are localized at atomic sites, this configuration can be destroyed by strong enough external effects. Therefore, in reality, topological protection does not hold. Additionally, the strict easy-plane model is not realistic because anisotropies are finite, and so the chiral pair of walls may also be destroyed if the spins rotate in the third dimension.

Lastly, we have presented some characteristics and properties of magnetic skyrmions. These topological solitons arise in a system from the introduction of a lengthscale via competing interactions. In two dimensions of space, they are localized, topologically nontrivial spin textures. If a spin spiral ground state can be realized from only interspin interactions, additional couplings such as magnetic anisotropies and external magnetic fields can then suppress the SS state to recover the uniform FM state, from which isolated skyrmions may be stabilized as metastable excitations. Under an external magnetic field, a skyrmion lattice arises at intermediate field values. Depending on the helicity of the spin texture, we distinguish between Néel and Block skyrmions. Néel skyrmions possess a magnetization profile similar to that of a chiral pair of Néel walls along any radial direction, resulting in a helicity of $\phi_{0}=0$ or $\pi$, while Bloch skyrmions possess a profile similar to a chiral pair of Bloch walls, with helicity $\phi_{0}=\pi / 2$ or $3 \pi / 2$. Amongst topologically nontrivial spin textures in two dimensions, solutions formally called skyrmions corresponds to the case where the winding direction of the spins matches the spatial travelling direction when travelling around a circular contour at a fixed distance to the skyrmion core, yielding a positive vorticity, $n>0$. In the case in which the spins rotate in the direction opposite to the travelling direction, the spin texture is an antiskyrmion with negative vorticity, $n<0$. In that case, the magnetization profile along a radial direction alternates between Néel-type and Bloch-type.

Chiral magnetic skyrmions are skyrmions stabilized by the DMI, and are therefore characterized by a given sense of rotation of the spins along a radial direction. The type of the DMI also selects a particular category of skyrmion solutions. Néel skyrmions are stabilized by interfacial DMI, which is typically found in systems such 
as $\mathrm{Ir} / \mathrm{Co} / \mathrm{Pt}, \mathrm{Pt} / \mathrm{Co} / \mathrm{AlO}_{x}$, and $\mathrm{Pd} / \mathrm{Fe} / \mathrm{Ir}(111)$. Bloch skyrmions are stabilized by bulk DMI, reported, for instance, in the bulk of $\mathrm{MnSi}$, and antiskyrmions are stabilized by anisotropic DMI, which was reported in tetragonal Heusler compounds. Chiral skyrmions possess a strongly localized core, with linear variations of the polar angle along the radial direction, while the asymptotic profile of the tail far from the centre resembles that of a ferromagnetic domain wall.

Another stabilization mechanism of skyrmions is found in frustrated Heisenberg exchange, in which case, for instance, the exchange is ferromagnetic between first neighbours, but antiferromagnetic beyond first neighbours. Skyrmions stabilized by frustrated exchange possess a helicity degree of freedom, and in the absence of DMI, skyrmions and antiskyrmions are degenerate in energy. The skyrmion core is strongly localized, while, in the tail, the polar angle oscillates as a function of distance, indicating a reversal of helicity. While the interaction potential between chiral skyrmions is only repulsive, the oscillations of the polar angle entails that the pairwise interaction potential between skyrmions in frustrated magnets alternates between repulsive and attractive.

Finally, skyrmion bubbles, or bubbles domains, can be stabilized by dipolar couplings. Although they carry a topological charge, they do not exhibit the same topological properties as skyrmions and tend to be much bigger, with a weakly localized core.

In the rest of this thesis, we will focus on ultrathin, perpendicularly magnetized films, so that, in simulations, we can restrict ourselves to two spatial dimensions, and the dipolar energy contribution may be taken as an effective anisotropy. In thin magnetic films $(\mathrm{Co}, \mathrm{Fe})$, the breaking of the structural inversion symmetry at an interface with a heavy-metal exhibiting a strong SOC (Ir, Pd) gives rise to an interfacial type of DMI, which in turns favours cycloidal magnetic textures and Néel skyrmions. This is the type of system currently most envisioned for future spintronics applications, in which isolated magnetic skyrmions are to be used as movable data bits. For such technological applications, a precise knowledge and understanding of the average lifetime of isolated skyrmions at room temperature is necessary. Hence, in what follows, we tackle the task of estimating the thermal stability of metastable magnetic skyrmions, with particular emphasis on nanoscale chiral skyrmions stabilized, in most cases, by interfacial DMI. The material parameters used in Chapters 4 and 5 of the present thesis partially correspond to $\mathrm{Pt} / \mathrm{Co} / \mathrm{AlO}_{x}$ samples, which is one of the extensively studied systems where nanoscale skyrmions have been experimentally observed. Additionally, frustrated exchange was predicted to arise at certain types of interfaces by DFT calculations, most notably in $\mathrm{Pd}_{\mathrm{fcc}} / \mathrm{Fe}, \mathrm{Rh} / \mathrm{Fe}$, and $\mathrm{Rh} / \mathrm{Co}$ bilayers on $\operatorname{Ir}(111)$. Therefore, we 
shall also study the thermal stability of skyrmions in a frustrated magnet in Chapter 6 , as it may well be relevant for applications. 


\section{Chapter 3}

\section{Langer's theory and application to magnetic spin systems}

\subsection{Introduction}

\subsubsection{Preamble: on the theory of Brownian motion}

The first account of Brownian motion is attributed to the botanist Robert Brown in 1827 [120] who observed aqueous suspensions of pollen grains under a microscope, and found that the grains were performing "rapid oscillatory motion". Throughout the nineteenth century, this motion of a so-called Brownian particle immersed in a solvent sparked many investigations and speculations regarding its origin, and the following properties emerged:

- the trajectories are highly irregular and non-differentiable;

- the motion is independent of the nature of the particle: it is a universal phenomenon of mechanical origin;

- the motion never ceases as constant energy is injected into it, and thus pertains to the realm of out-of-equilibrium dynamics;

- the smaller the particles, the higher the temperature, and the higher the viscosity of the fluid, the more active the motion.

As is well-known, it was Einstein who, in 1905, initiated the theory of Brownian motion by combining the random walk process with the Maxwell-Boltzmann distribution [121]. He attributed the cause of the motion to the thermal fluctuations of the molecules of the solvent. In a frictionless fluid, a collision with a molecule of the solvent would modify the velocity of the particle. However, in a viscous fluid, the change 
in velocity is rapidly dissipated, and the result is a change in the position of the particle. Einstein assumed that the overall result of the collisions is a kind of random walk of the particle. The interplay of the injection of energy through collision, followed by its dissipation through the fluid viscosity, is an example of what is known as the fluctuation-dissipation theorem [122].

Consider a small particle of mass $M$ immersed in a solvent at temperature $T$. The following discussions are carried out in one dimension without loss of generality, and the extension to higher dimensions is straightforward. Formally, the microscopic treatment of the system would involve the Brownian particle and all the molecules of the solvent. This is an $N$-body problem, where $N \approx 10^{23}$, which therefore cannot be solved analytically. From the equipartition theorem in one dimension, the mean energy of the particle is [48]

$$
\frac{1}{2} M\left\langle v^{2}\right\rangle=\frac{1}{2} k_{B} T
$$

where $v$ is the velocity of the particle, $k_{B}$ is Boltzmann's constant, and $T$ is the absolute temperature. Let the the molecules of the solvent be of mass $m$, with $m \ll M$, then the thermal velocity of the Brownian particle satisfies

$$
v_{B P}=\sqrt{\left\langle v^{2}\right\rangle}=\sqrt{k_{B} T / M}=v_{\text {solvent }} \sqrt{m / M} \ll v_{\text {solvent }} .
$$

If follows that the position, $x$, of the particle is a slow variable on the timescale of the solvent. Einstein introduced a "coarse-graining" which eliminates the rapid degrees of freedom of the solvent and leads to the apparent stochastic nature of the motion. The solvent is then treated as a structureless medium with only a temperature $T$. Stochasticity emerges because, if we were to consider a second system of the particle immersed in the fluid, identical to the first system in all aspects but the initial conditions of the molecules in the fluid, then the motion of the particle would be different. We consider an ensemble of such systems, known as a Gibbs ensemble. While we cannot predict the time-evolution of the (stochastic) macroscopic observables of the system, we can predict that of ensemble averages. For instance, the probability to find the position of the particle in the interval $(x, x+d x)$ is equivalent to the number of systems of the ensemble with positions found in the interval $(x, x+d x)$ divided by the number of systems in the ensemble. Since $x$ is a continuous variable, let $W(x, t)$ be a probability density, also known in the literature as a probability distribution function of the variable $x$. Its time evolution obeys a class of equations known as the Fokker-Planck equation (FPE), which, in one dimension, takes the form [48]

$$
\frac{\partial W}{\partial t}=\left[\frac{\partial}{\partial x} D^{(1)}(x)+\frac{\partial^{2}}{\partial x^{2}} D^{(2)}(x)\right] W .
$$


In Eq. $(3.3), D^{(1)}(x)$ is the drift coefficient, of deterministic origin, and $D^{(2)}(x)$ is the diffusion coefficient, of stochastic origin. The FPE represents an equation of motion for the distribution function.

In 1908, Langevin [123] postulated that the velocity $v=d x / d t=\dot{x}$ must also be a slow variable on the timescale of the solvent, and thus proposed a mechanistic description of Brownian motion. A Brownian particle in a solvent will, first of all, feel a friction force, whose expression is given by Stokes' law, $F_{c}=-\zeta v$, where $\zeta$ is the friction coefficient. Furthermore, Langevin introduced a stochastic fluctuating force $F_{f}(t)$ in the form of Gaussian white noise. That force contains all contributions from the rapid degrees of freedom eliminated by the coarse-graining. Using Newton's second law of motion and dividing by the mass $M$ of the particle, we arrive at Langevin's equation for the motion of a free Brownian particle,

$$
\dot{v}=-\gamma v+\Gamma(t)
$$

where $\dot{v}=d v / d t, \gamma=\zeta / M$, and $\Gamma(t)=F_{f}(t) / M$ is a fluctuating force per unit mass called the Langevin force. Its statistical properties are as follows. Firstly,

$$
\langle\Gamma(t)\rangle=0
$$

because on average, $\langle v(t)\rangle$ should obey the deterministic version of Eq. (3.4) obtained by setting $\Gamma(t)=0$. The symbol $\langle\ldots\rangle$ corresponds to an ensemble average. Secondly, we suppose that the collisions with different molecules of the fluid should be independent, so that if we multiply two Langevin forces at different times, $t$ and $t^{\prime}$, the average value is zero if the time difference $\left|t-t^{\prime}\right|$ is larger than the average duration of a collision, $\tau_{0}$, i.e., $\tau_{0} \ll\left|t-t^{\prime}\right|$. Furthermore, $\tau_{0}$ is usually much smaller than the relaxation time $\tau=1 / \gamma$, and so we take the limit $\tau_{0} \rightarrow 0$, which yields,

$$
\left\langle\Gamma(t) \Gamma\left(t^{\prime}\right)\right\rangle=2 \gamma k_{B} T \delta\left(t-t^{\prime}\right),
$$

where $\delta\left(t-t^{\prime}\right)$ is the Dirac distribution. Since $\Gamma(t)$ is a Gaussian process, it is completely characterized by (3.5) and (3.6). We can note that, although Einstein assumed that the trajectory is non-differentiable, Langevin could define a velocity $v$ by considering a finer timescale for the coarse-graining. If the noise $\Gamma(t)$ does not depend on $x$, it is called additive. On the other hand, if it does depend on $x$, it is called multiplicative noise, and requires an appropriate interpretation. 


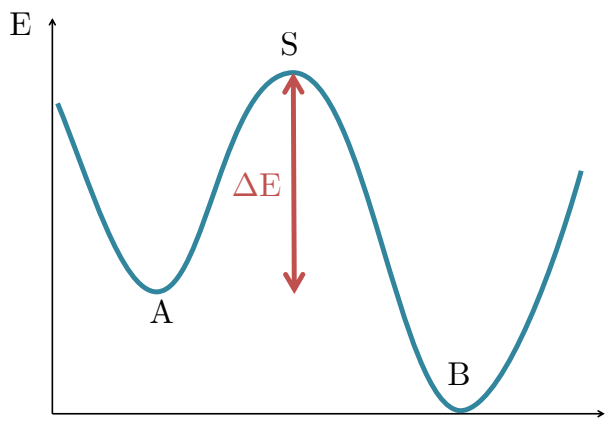

Reaction coordinate

(a)

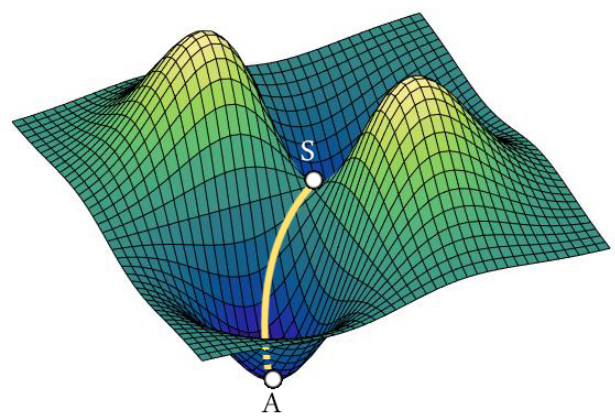

(b)

Figure 3.1: Example energy surface of a system possessing a metastable local minimum $A$ and a lower energy minimum $B$, separated by an energy barrier at $S$. The case (a) can be viewed as the one dimensional system treated by Kramers, or a projection of (b) along the reaction coordinate. The case (b) is an example energy landscape for a system possessing two degrees of freedom. In that case, the top of the barrier is a saddle point. The reaction coordinate corresponds to the path of minimum energy connecting $A, S$ and $M$. If the saddle point is of first order, it constitutes a maximum of the energy along the reaction coordinate, but a minimum along all the other degrees of freedom.

\subsubsection{Reaction rate theory}

Modern reaction rate theory initially developed independently from the theory of Brownian motion. Its origin dates back to the 1880s, when Arrhenius [50] first described the rate coefficient of a chemical reaction as

$$
k=f_{0} e^{-\Delta E / k_{B} T},
$$

where $\Delta E$ is the activation energy, and $f_{0}$ is a prefactor. This equation subsequently led to the representation of chemical reactions as an ensemble of particles situated at the bottom of a potential well $A$ (Fig. 3.1a). Over time, under the influence of thermal agitation, rare particles may escape over a barrier of height $\Delta E \gg k_{B} T$ at $S$, and never return to $A$. In the context of transition state theory (TST), the Arrhenius law is expressed as [50],

$$
k_{\mathrm{TST}}=\frac{\omega_{A}}{2 \pi} e^{-\beta \Delta E},
$$

where $\beta=1 / k_{B} T$ is Boltzmann's constant. The attempt frequency $\omega_{A}$ is the angular frequency of a particle performing oscillatory motion at the bottom of the potential well. The exponential Boltzmann factor weighs the escape from the well.

Reaction rate theory was set in the context of non-equilibrium statistical mechanics in 1940 by the work of Kramers [82]. He chose to model a chemical reaction as a 
classical particle moving in a one dimensional potential $E(x)$, in which $x$ is the reaction coordinate. The particle is coupled to its environment in the form of a heat bath which remains in perpetual thermal equilibrium at temperature $T$. The coupling to the heat bath is modelled by the Brownian motion performed by the particle [121], which represents all the remaining degrees of freedom of the total system consisting of the particle and the heat bath. Kramers assumed that a (Gibbs) ensemble of particles are initially trapped in the potential well near $A$. They receive energy from their surroundings, such that a Maxwell-Boltzmann distribution is rapidly attained in the well. That is, $W(x, t) \sim e^{-\beta E(x)}$, so that, in other words, the number of particles with energy found between $E$ and $E+d E$ is proportional to $e^{-\beta E(x)} d E$. Over time, rare particles may gain energy in excess of the barrier height and escape over the barrier at $S$ to reach the lower energy minimum $B$, never to return. The diffusion over the barrier is assumed to be a quasi-stationary process, so that the disturbance to the Maxwell-Boltzmann distribution can be neglected at all times. The rate constant corresponds to the average number of particles of the ensemble that cross the barrier per unit time. Kramers' objective was to derive a prefactor from a microscopic model of the reaction system, and insert it into the TST result. The Kramers formula for the escape from an isolated well (no returns from $B$ ) takes the form,

$$
k_{\mathrm{Kr}}=A \frac{\omega_{A}}{2 \pi} e^{-\beta \Delta E},
$$

where $A$ contains non-equilibrium effects caused by the disturbance to the MaxwellBoltzmann distribution at the barrier top, and describes deviations from the TST rate in Eq. (3.8). That model is purely classical, and yields explicit formulas for the rate for very low, and intermediate-to-high dissipative coupling to the heat bath.

In 1969, Langer [49] proposed the most complete treatment of the extension of Kramers theory to a multidimensional phase space in the intermediate-to-high damping (IHD) regime [47]. His treatment is a generalization of a 1935 calculation by Becker and Döring of the rate of condensation of a supersaturated vapour [124]. According to Langer, if the characteristic disturbance that leads to the transition appears spontaneously as a thermodynamic fluctuation, it is an intrinsic property of the system and is classified as a homogeneous disturbance. On the other hand, if it is caused by a foreign object that is not part of the system, such as the presence of defects in the wall of the container, it is classified as inhomogeneous, of lesser fundamental interest. It is the former that was treated by Langer in his statistical theory for the decay of metastable states. The extension to many degrees of freedom makes the theory applicable to magnetic spin systems, with interactions such as exchange and dipole-dipole couplings. Within a multidimensional energy surface, (meta)stable states correspond to (local) energy minima with respect to all degrees of freedom. The internal energy barrier, $\Delta E$, 
is linked to the most probable path(s) through the energy landscape, in the form of minimum energy paths (MEPs), which usually traverse a first-order saddle point (SP). First-order saddle points represent a minimum of the energy with respect to all degrees of freedom, except one - the reaction coordinate, which connects states $A, S$, and $B$. This is illustrated in Fig. $3.1 \mathrm{~b}$. If several saddle points are present in the energy landscape, and the different transition processes can be considered independent from one another, the total escape rate out of $A$ is a priori given by the sum of the escape rates over each saddle point.

The exponential Boltzmann factor in equation (3.7) shows that the decay of a metastable state takes place over time scales which are much longer than the time scales linked with the intrinsic dynamics of the system. For this reason, solely understanding the dynamics of a system is not enough in order to predict the process(es) by which it decays, and it is essential to study and understand the decay mechanisms themselves. For systems with many degrees of freedom and many-body interactions such as magnetic spin systems, this is often an arduous task, and relying on numerical schemes becomes almost unavoidable. The difficulty in computing transition rates for such a class of systems thus lies in the precise identification of the first-order saddle point(s) on the energy landscape on the one hand, and on the correct evaluation of the rate prefactor, $f_{0}$, on the other hand.

In this chapter, we firstly give the scope of Langer's theory and how it can be applied to magnetic spins systems in Sec. 3.2. In that context, we detail the calculation of the main contributions entering in the rate prefactor. We then describe the GNEB method [59] in Sec. 3.4, which enables the numerical computation of a minimum energy path on the complex multivariable energy surface, and the precise identification of the firstorder saddle point on the path. Finally, in Sec. 3.5, we give details of the numerical implementation of the different building blocks in Langer's theory on the atomistic spin lattice.

\subsection{Langer's theory in the IHD regime}

\subsubsection{Framework for the derivation of the rate expression}

Langer's theory describes the rate of decay of a metastable state under the effect of thermal fluctuations, provided certain conditions are fulfilled. It is set within the IHD regime, and the other main assumptions essentially mirror that of Kramers [82]. The barrier height must be large compared to thermal fluctuations, $\Delta E \gg k_{B} T$, so that the diffusion over the barrier is slow enough as to ensure that a Maxwell-Boltzmann 
distribution is rapidly attained and maintained in the metastable well at all times. It follows that the contribution to the flux over the saddle point will come mainly from a small region around $S[47]$. This also ensures that barrier re-crossing events are negligible, so that state $B$ is viewed as a sink of probability. According to Ref. [47], $\Delta E \geq 5 k_{B} T$ seems to be a reasonable lower bound for the energy barrier. Additionally, we postulate small amplitudes of the fluctuations about the extrema states $A$ and $S$, so that the energy surface in their vicinity can be approximated as a quasiharmonic potential. This assumption becomes more accurate at low $T$.

The Hamiltonian $E(\eta)$ is function of $2 N$ variables $\eta=\left\{\eta_{1}, \eta_{2}, \ldots \eta_{2 N}\right\}$, which could be coordinates and associated conjugate momenta of a point in phase space, or coordinates describing the orientation of magnetic vectors. $E(\eta)$ possesses two minima, $A$ and $B$, separated by a saddle point $S$, and $E\left(\eta^{A}\right)>E\left(\eta^{B}\right)$. We assume that the energy in the vicinity of the extrema states can be approximated as a Taylor series truncated to the second order term,

$$
E(\eta) \sim E^{0}(\tilde{\eta})+\frac{1}{2}(\eta-\tilde{\eta}) H_{\tilde{\eta}}(\eta-\tilde{\eta})^{T},
$$

where $\tilde{\eta}=\left(\tilde{\eta}_{1} \ldots \tilde{\eta}_{2 N}\right)$ are the coordinates of a local extremum (the local minimum $A$ or saddle point $S)$. At the extrema,

$$
\frac{\partial E}{\partial \eta}_{\tilde{\eta}}=0
$$

in which the notation ${ }_{\mid \tilde{\eta}}$ means the expression is evaluated at $\tilde{\eta}$, and

$$
H_{\tilde{\eta}}=\left(\begin{array}{ccc}
\left.\frac{\partial^{2} E}{\partial \eta_{1}^{2}}\right|_{\tilde{\eta}} & \cdots & \frac{\partial^{2} E}{\left.\partial \eta_{1} \partial \eta_{2 N}\right|_{\tilde{\eta}}} \\
\vdots & & \vdots \\
\left.\frac{\partial^{2} E}{\partial \eta_{2 N} \partial \eta_{1}}\right|_{\tilde{\eta}} & \cdots & \frac{\partial^{2} E}{\left.\partial \eta_{2 N}^{2}\right|_{\tilde{\eta}}}
\end{array}\right),
$$

is the energy Hessian evaluated at $\tilde{\eta}$, which contains the second derivatives of the energy with respect to all degrees of freedom. It is symmetric and real, and therefore Hermitian by construction, which ensures that it admits only real eigenvectors and eigenvalues. The Hessian can be diagonalized by expanding small fluctuations about the extrema in the eigenbasis,

$$
\eta_{i}-\tilde{\eta}_{i}=a_{i} \chi_{i}
$$

where $\left\{a_{i}\right\}, i=1 \ldots 2 N$ is a set of eigenbasis coordinates characterizing the amplitudes of the fluctuations, and $\left\{\chi_{i}\right\}$ is a set of orthonormal eigenvectors forming a complete 
basis in the space of configurations. They satisfy the eigenvalue equation,

$$
H \chi_{i}=\lambda_{i} \chi_{i}
$$

where the $\left\{\lambda_{i}\right\}$ are the eigenvalues of the Hessian. Because $H$ is evaluated at energy extrema, the $\left\{\lambda_{i}\right\}$ correspond to the $2 N$ curvatures of the energy landscape. From Eqs. (3.10), (3.13), and (3.14), the energy of small deviations from an extremum can be linearized as

$$
E(\eta) \sim E^{0}(\tilde{\eta})+\frac{1}{2} \sum_{i} \lambda_{i} a_{i}^{2} .
$$

In what follows, we mainly follow the notations from [47, 50, 125]. The following assumptions are made about the density of states of the system, $\rho(\eta, t)$.

1. The time evolution of of the density of states $\rho(\eta, t)$ obeys a Fokker-Plank equation,

$$
\frac{\partial \rho(\eta, t)}{\partial t}=\sum_{i=1}^{2 N} \sum_{j=1}^{2 N} \frac{\partial}{\partial \eta_{i}} M_{i j}\left[\frac{\partial E}{\partial \eta_{j}}+k_{B} T \frac{\partial}{\partial \eta_{j}}\right] \rho(\eta, t),
$$

where $\mathbf{M}=\left(M_{i j}\right)$ is the transport matrix, which we assume to be constant. $\mathbf{M}$ has a symmetric part $\mathbf{D}=\frac{1}{2}\left(\mathbf{M}+\mathbf{M}^{T}\right)$, referred to as the diffusion matrix, which characterizes the thermal fluctuations due to the heat bath, and an antisymmetric part $\mathbf{A}=\frac{1}{2}\left(\mathbf{M}-\mathbf{M}^{T}\right)$, which describes the motion in the absence of the heat bath. Eq. (3.16) is linearized about the saddle point $\eta^{S}$ by inserting (3.10) into (3.16), which yields

$$
\frac{\partial \rho}{\partial t}=\sum_{i j} \frac{\partial}{\partial \eta_{i}} M_{i j}\left[\sum_{k} H_{j k}^{S}\left(\eta_{k}-\eta_{k}^{S}\right)+k_{B} T \frac{\partial}{\partial \eta_{j}}\right] \rho(\eta),
$$

in which the $H_{j k}^{S}$ are entries in the Hessian matrix evaluated at $S$. The FPE (Eq. 3.17) can be interpreted as a continuity equation of the $2 \mathrm{~N}$-dimensional probability current density $J_{i}(\eta, t)$ as

$$
\frac{\partial \rho}{\partial t}=\sum_{i} \frac{\partial J_{i}}{\partial \eta_{i}}
$$

where

$$
J_{i}=\sum_{j=1}^{2 N} M_{i j}\left[\sum_{k} H_{j k}^{S}\left(\eta_{k}-\eta_{k}^{S}\right)+k_{B} T \frac{\partial}{\partial \eta_{j}}\right] \rho(\eta, t)
$$


Since we assumed quasi-equilibrium,

$$
\frac{\partial \rho}{\partial t}=0
$$

2. The high barrier ensures that, in the vicinity of $A$, the density of states corresponds to the Maxwell-Boltzmann distribution,

$$
\rho(\eta)=\rho_{e q}(\eta)=\frac{1}{Z} e^{-\beta E(\eta)}, \quad \quad \eta \sim \eta^{A}
$$

where

$$
Z=\int_{-\infty}^{\infty} \ldots \int_{-\infty}^{\infty} e^{-\beta E} d \eta_{1} \ldots d \eta_{2 N}
$$

is the partition function.

3. Practically no particles have reached the lower minimum $S$, so we have the sink boundary condition

$$
\rho(\eta)=0, \quad \eta \text { beyond } \eta^{S} \text {. }
$$

Although the system is assumed to be at quasi-equilibrium, there is a weak current of rare particles over the barrier, in which case the density of states is written as

$$
\rho(\eta)=\zeta(\eta) \rho_{e q}(\eta)
$$

$\zeta$ is the crossover function which characterizes the diffusion over the barrier. It is expressed as an error function of a single variable $u$ as

$$
\zeta(\eta)=\zeta(u)=\frac{1}{2 \pi k_{B} T} \int_{u}^{\infty} \exp \left(-\frac{\beta z^{2}}{2}\right) d z
$$

where $u$ is a linear combination of the $\eta_{i}$ 's,

$$
u=\sum_{i=1}^{2 N} U_{i}\left(\eta_{i}-\eta_{i}^{S}\right)
$$

with the $U_{i}$ 's chosen as constants. The rate $k$ depends on $j$, the flux of particles over the barrier, and $n_{A}$, the number of particles in the metastable well, that is,

$$
k=\frac{j}{n_{A}} .
$$

The expression for the flux of particles is calculated [47,49] by integrating the current 


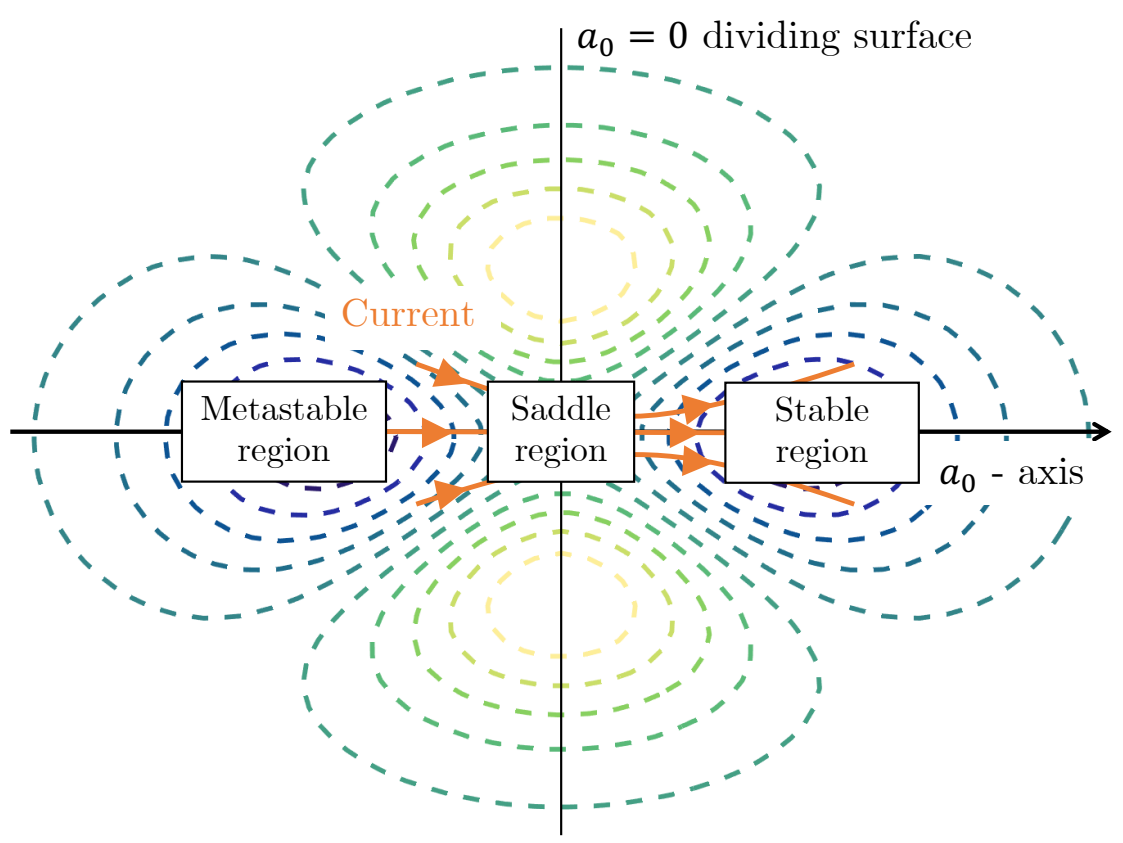

Figure 3.2: Schematic view of the energy surface where a metastable region located at $a_{0}<0$ is separated from a more stable region $\left(a_{0}>0\right)$ by a saddle. $a_{0}$ is the eigenbasis coordinate associated with the unstable mode at the top of the barrier and represents the reaction coordinate. A weak current of particles moving in the direction of increasing $a_{0}$, where each particle represents the entire system, originates from a small region around the saddle point and reaches the more stable region. The rate $k$ of the transition is given by the ratio of the current over the barrier and the number of particles in the metastable region.

density (Eqs. (3.18) and (3.17)) through the plane $u=0$ as

$$
j=\sum_{i} \int_{u=0} J_{i}(\eta) d S_{i}
$$

$u$ is, in fact, parametrized by $a_{0}$, which is the eigenbasis coordinate associated with the unstable mode at the top of the barrier, and corresponds to the reaction coordinate. By convention, the dividing surface defined by $a_{0}=0$ passes through the saddle point, while the metastable (saddle) region is found at negative (positive) values of $a_{0}$. This is represented in a schematic view of the energy landscape in Fig. 3.2. The corresponding eigenvector $\chi_{0}$ gives the direction of the current. 
The number of particles in the well, $n_{A}$, is calculated by integrating the density of states in the well (Eq. 3.21),

$$
\begin{aligned}
n_{A} & =\int \ldots \int \rho_{\mathrm{eq}} d \eta_{1} \ldots d \eta_{2 N} \\
& =Z^{-1} \int \ldots \int e^{-\beta E_{A}} d \eta_{1} \ldots d \eta_{2 N},
\end{aligned}
$$

in which the partition function, $Z$, was previously defined in Eq. (3.22). In the previous expression, $E_{A}=E\left(\eta^{A}\right)$ is subsequently linearized according to Eq. (3.15). This term, as well as (3.28), yield products of Gaussian integrals of the form,

$$
\prod_{i=1}^{2 N} \int_{-\infty}^{+\infty} \exp \left(-\frac{\beta}{2} \lambda_{i} a_{i}^{2}\right) d a_{i}=\prod_{i=1}^{2 N} \sqrt{\frac{2 \pi}{\beta \lambda_{i}}} .
$$

In the end, the total rate of escape out of state $A$ is expressed as

$$
k=\frac{\lambda_{+}}{2 \pi} \Omega_{0} e^{-\beta \Delta E}=\frac{\lambda_{+}}{2 \pi} \sqrt{\frac{\operatorname{det} H^{A}}{\left|\operatorname{det} H^{S}\right|}} e^{-\beta \Delta E},
$$

where $\Omega_{0}$ is the ratio of energy curvatures in the metastable well and at the saddle point, and $\lambda_{+}$is the dynamical contribution that takes into account the dissipative dynamics of the system at the top of the barrier, and constitutes a correction from TST rates. This results pertains to the IHD regime due to the quasiharmonic approximation of the energy that was performed in the vicinity of the extrema. At high damping, the system relaxes faster and explores less of its phase space. Low damping, on the other hand, implies that the system takes longer to relax back to the equilibrium state after a perturbation, and consequently visits a wider area of its phase space. At very low damping, the region where the density of states deviates from the Maxwell-Boltzmann distribution - i.e. the region where the system deviates from equilibrium - extends beyond the small region around the saddle point in which the potential may be replaced by a quadratic approximation, so the theory fails.

The meaning and derivation of the terms in Eq. (3.31) for magnetic spin systems are discussed in what follows. The escape rate may also be written in terms of the partition functions around the states $A$ and $S$, respectively $Z_{A}$ and $Z_{S}$ as [126]

$$
k=\frac{\lambda+Z_{S}}{2 \pi} \frac{Z_{A}}{Z_{A}}
$$




\subsubsection{Ratio of energy curvatures: interpretation and the case of Gold- stone modes}

When evaluated at an extremum, the $\left\{\lambda_{i}\right\}$ correspond to the $2 N$ curvatures of the energy surface in normal mode space. A positive (negative) curvature corresponds to a mode of stable (unstable) fluctuations. A zero-curvature corresponds to a Goldstone mode of zero energy fluctuation, and is associated with a continuous unbroken global symmetry [49, 52]. In the case of a first-order saddle point, all curvatures at $A$ and $S$ are either positive or zero-curvatures, aside from a single negative curvature at the top of the barrier. This unstable mode is the one that will eventually allow the system overcome the barrier and reach the lower energy minimum.

In the absence of Goldstone modes, the factor $\Omega_{0}$ in Eq. (3.31) is obtained from the square root of the product of the curvatures of the energy at states $A$ and $S$,

$$
\Omega_{0}=\sqrt{\frac{\operatorname{det} H^{A}}{\left|\operatorname{det} H^{S}\right|}}=\sqrt{\frac{\prod_{i} \lambda_{i}^{A}}{\prod_{j}\left|\lambda_{j}^{S}\right|}} .
$$

\section{The case of zero-curvatures}

If the Hessian contains one or several zero-eigenvalue(s) at $A$ and/or $S$, the contribution of the zero-mode(s) to the prefactor needs to be handled separately. The zeroeigenvalue is removed from the determinant of the Hessian, and instead of a Gaussian integral (Eq. (3.30)), we get a contribution of the type

$$
\int d a_{l}
$$

where $a_{l}$ is the eigenbasis coordinate associated with the zero-eigenvalue $\lambda_{l}$, and the integration is performed over the domain of $a_{l}$. If this domain is finite, we get a finite contribution to the prefactor. If we assume we have $k$ zero-eigenvalues in the Hessian, we get,

$$
v=\int d a_{l_{1}} \int d a_{l_{2}} \ldots \int d a_{l_{k}},
$$

where $v$ is the volume of the $k$-dimensional subspace associated with the variables $\left\{a_{l_{j}}\right\}$, $j=1 \ldots k$, that correspond to zero-eigenvalues. Additionally, a factor $(2 \pi / \beta)^{-1 / 2}$ appears for each Goldstone mode at $S$, and $(2 \pi / \beta)^{1 / 2}$ appears for each Goldstone mode at $A$. This is because the $(2 \pi / \beta)^{1 / 2}$ factors in Eq. $(3.30)$ no longer cancel out if the number of Gaussian integrals entering in the final expression of $\Omega_{0}$ differs between $A$ and $S$. In other words, if the number of Goldstone modes is non-zero and different at $A$ and $S$, the attempt frequency obtains a temperature-dependence. Hence, in the more general 
case where there are $k_{A}$ zero-curvatures at $\mathrm{A}$ and $k_{S}$ zero-curvatures at $\mathrm{S}, \mathrm{Eq} .(3.33)$ becomes

$$
\Omega_{0}=\left(\frac{2 \pi}{\beta}\right)^{\left(k_{A}-k_{S}\right) / 2} \sqrt{\frac{\operatorname{det}^{\prime} H^{A}}{\left|\operatorname{det}^{\prime} H^{S}\right|}} \frac{v_{S}}{v_{A}},
$$

where $\operatorname{det}^{\prime}$ is a determinant calculated after removing the zero-eigenvalues, and $v_{A}, v_{S}$ are the volumes associated with the Goldstone modes at $A$ and $S$.

Langer's theory treatments of rotational and translational Goldstone modes in magnetic spin systems were previously carried out in works by Braun [52] and Loxley [126]. We can also mention the more recent Refs. [63, 109] where it was done numerically in the context of skyrmion lifetimes. Although the authors used a different version of reaction rate theory, namely Harmonic TST (HTST) [127], the extension of HTST to include Goldstone modes is the same as that of Langer's theory. Eq. (3.36) should also come with some form of criterion for when an eigenmode should be treated as a Goldstone mode. Numerically - and experimentally - an energy curvature of exactly zero will in principle not exist. An intuitive idea for such a criterion is that a curvature will appear flat to the system if it is small compared to the amplitude of thermal fluctuations. For translational Goldstone modes, the limit [109,128]

$$
\frac{1}{2} \lambda_{\mathrm{tr}} v^{2} \lesssim k_{B} T
$$

where $\lambda_{\mathrm{tr}} \approx 0$ is an eigenvalue associated with a translation mode, was proposed.

\section{Change in entropy}

To interpret the physical meaning behind $\Omega_{0}$, we examine Eq. (3.33). The product of curvatures evaluated at an extremum in the energy surface, $\left(\prod_{i} \lambda_{i}^{A, S}\right)^{-1}$, can be seen as a measure of the total volume of configuration space $(\eta$-space) accessible to thermal fluctuations in that particular state. The ratio of eigenvalues thus corresponds to the change in that volume induced by the transition from $A$ to $S$. In other words, it characterizes the relative volume of the saddle point region. $\Omega_{0} \ll 1$ is interpreted as a large volume of the metastable well, and/or a narrow saddle region in $\eta$-space (eg: Fig. 3.1b). As a consequence, the probability that the system will visit the saddle region is low. These considerations bring us to the notion of entropy, which measures the number of micro-realisations which exist for a given macrostate, and is also commonly interpreted as a measure of disorder. As entropy is normally defined for a stable equilibrium state, we define the change in configurational entropy $\Delta S$ with respect to stable 
fluctuations only. If the saddle point is of first order, this corresponds to [126]

$$
e^{\Delta S / k_{B}} \equiv \sqrt{\frac{\beta}{2 \pi}} \sqrt{\frac{\prod_{i} \lambda_{i}^{A}}{\prod_{j}^{\prime} \lambda_{j}^{S}}},
$$

where $\prod^{\prime}$ is defined for positive curvatures, and an additional $(\beta / 2 \pi)^{1 / 2}$ factor is needed to keep the dimension consistent. The total activation energy corresponds to the change in Helmholtz free energy, and takes into account the change in internal energy, $\Delta E$, and the change in entropy, $\Delta S$, such that $\Delta F=\Delta E-T \Delta S$. It follows that Eq. (3.33) can be expressed as

$$
\Omega_{0}=\sqrt{\frac{2 \pi}{\beta\left|\lambda_{1}^{S}\right|}} e^{\Delta S / k_{B}},
$$

in which $\lambda_{1}^{S}$ is the negative curvature at $S$. The factor $\Omega_{0}$ is thus a measure of the number of available configurations, and gives the entropic contribution to the prefactor. More generally, if the saddle point is of order $k$, i.e., if $k$ unstable modes exist at $S$, Eqs. (3.38) and (3.39) may be generalized as

$$
e^{\Delta S / k_{B}} \equiv\left(\frac{\beta}{2 \pi}\right)^{k / 2} \sqrt{\frac{\prod_{i} \lambda_{i}^{A}}{\prod_{j}^{\prime} \lambda_{j}^{S}}},
$$

and

$$
\Omega_{0}=\left(\frac{2 \pi}{\beta}\right)^{k / 2} \sqrt{\prod_{j}^{\prime \prime}\left|\lambda_{j}^{S}\right|^{-1}} e^{\Delta S / k_{B}},
$$

in which $\prod^{\prime \prime}$ is a product over negative curvatures.

\subsection{Application to magnetic spin systems}

In what follows, we consider a system of $N$ magnetic spins on a lattice, for which the change in their magnitude is generally much faster than the change in their orientation. This separation of timescales justifies the assumption that the amplitudes of the moments remain constant. The system can thus be described in terms of orientations of the moments alone, and the energy surface reduces to a $2 \mathrm{~N}$-dimensional landscape. Below, we provide details of the derivation of the different contributions to the rate prefactor of Langer's theory, in the context of its application to a system of magnetic spins. The restriction to the IHD regime means that the scope of the theory is limited to cases where the precessional dynamics can be neglected, in the sense that it does not significantly impact the transition path, and the time scale of the transition is set by the dissipation rate. 
One requirement of Kramers' method - and subsequently Langer's theory - is that the system may be modelled by the theory of Brownian motion. For magnetic systems, the environment acts a heat bath of constant temperature $T$. The magnetic vectors interact with the fast degrees of freedom of the environment in the form of phonons, conducting electrons, nuclear spins, etc. [68], leading to fluctuations in their orientation. The interactions of the spins with the environment may be modelled by a fast, fluctuating, stochastic magnetic field. The equation that governs the dynamics of the magnetic vectors, namely the Landau-Lifshitz-Gilbert equation (Eq. (3.46)), may be cast into a Langevin equation with multiplicative noise by including this term. This will be explored in more details in Chapter 5 .

In the past, Langer's theory was successfully applied to the analytical treatment of incoherent magnetization reversals by Braun in nanowires [52], and by Loxley and Stamps in soft/hard nanowires [53]. The first numerical implementations of the theory for magnetic spin systems are more recent and were performed in conjunction with the finite element method [62] for magnetization reversal problems in graded media grains.

\subsubsection{Construction of the energy Hessian in spherical coordinates}

The total energy of the system may be expressed in terms of spherical coordinates on the unit sphere $E(\boldsymbol{\theta}, \boldsymbol{\phi})$, where $\boldsymbol{\theta}=\left(\theta_{1} \ldots \theta_{N}\right)$ is the polar angle with respect to the Cartesian $z$-axis and $\phi=\left(\phi_{1} \ldots \phi_{N}\right)$ is the corresponding azimuth in the $x y$-plane (see Sec. 2.1). In principle, it is necessary to define canonically conjugate variables (p, $\mathbf{q})$ [47, 49,62], such that

$$
\begin{aligned}
& \mathbf{p}=\cos \theta \\
& \mathbf{q}=\boldsymbol{\phi}
\end{aligned}
$$

According to Langer's initial definitions, the total energy is a function of $N$ coordinates and $N$ canonically conjugate momenta [49]. However, even if the energy is only a function of the coordinates, the equipartition theorem holds [125]. The use of variables such as the ones defined in Eq. (3.42) ensures the Jacobian

$$
J_{i}=\operatorname{det} \frac{\partial\left(m_{i x}, m_{i y}, m_{i z}\right)}{\partial\left(p_{i}, q_{i}, 1\right)}=d p_{i} d q_{i}
$$

is not a function of $\left(\theta_{i}, \phi_{i}\right)$ [62]. In this thesis however, the Hessian matrix is constructed in spherical coordinates. This choice is simply a matter of convenience, as analytical expressions of the energy derivatives become heavy when using the change 
of variable $p=\cos \theta$. The choice of spherical coordinates requires corrections in the Hessian to take into account the spherical Jacobian, which we give in what follows. We define the spherical Hessian as

$$
H=\left(\begin{array}{ll}
H_{\theta \theta} & H_{\theta \phi} \\
H_{\phi \theta} & H_{\phi \phi}
\end{array}\right)
$$

in which

$$
\begin{aligned}
H_{\theta_{i} \theta_{j}} & =\frac{\partial^{2} E}{\partial \theta_{i} \partial \theta_{j}}, \\
H_{\theta_{i} \phi_{j}} & =\frac{1}{\sin \theta_{j}} \frac{\partial^{2} E}{\partial \theta_{i} \partial \phi_{j}}, \\
H_{\phi_{i} \theta_{j}} & =\frac{1}{\sin \theta_{i}} \frac{\partial^{2} E}{\partial \phi_{i} \partial \theta_{j}}, \\
H_{\phi_{i} \phi_{j}} & =\frac{1}{\sin \theta_{i} \sin \theta_{j}} \frac{\partial^{2} E}{\partial \phi_{i} \partial \phi_{j}} .
\end{aligned}
$$

Even though the total Hessian remains symmetric, it is necessary to remain cautious with the introduction of a DMI contribution as it makes the $H_{\theta \phi}$ submatrix non-symmetric. Therefore in general, $H_{\phi \theta}=H_{\theta \phi}^{T} \neq H_{\theta \phi}$, contrary to the way it was treated in Ref. [47].

\subsubsection{Determination of the dynamical prefactor $\lambda_{+}$}

The dynamical prefactor takes into account the dynamics of the system at the saddle point and is derived from the set deterministic Landau-Lifshitz-Gilbert (LLG) equations associated with each $\operatorname{spin} \mathbf{m}_{i}, i=1 \ldots N$ [47],

$$
\frac{d \mathbf{m}_{i}}{d t}=-\left[g^{\prime} \mathbf{m}_{i} \times \frac{\partial E}{\partial \mathbf{m}_{i}}+h^{\prime}\left(\mathbf{m}_{i} \times \frac{\partial E}{\partial \mathbf{m}_{i}}\right) \times \mathbf{m}_{i}\right],
$$

where

$$
g^{\prime}=\frac{\gamma_{e}}{\left(1+\alpha^{2}\right) M_{s}}
$$

corresponds to the gyromagnetic ratio $\gamma_{e}$ modified by a dimensionless damping factor $\alpha=\eta \gamma_{e} M_{s}$, in which $M_{s}$ is the saturation magnetization and $\eta$ is a damping parameter characterizing the coupling to the heat bath, and

$$
h^{\prime}=\alpha g^{\prime}
$$

It follows that the first term on the RHS of Eq. (3.46) is the Larmor equation describing the precession of the magnetization vector $\mathbf{m}_{i}$, and the second term is an alignment term whose effect is measured by $h^{\prime}$. The following derivation was adapted to spherical 
coordinates from [125]. We begin by reformulating Eq. (3.46) within the local spherical basis $\left(\mathbf{e}_{r}, \mathbf{e}_{\theta}, \mathbf{e}_{\phi}\right)$ in the following way:

$$
\begin{aligned}
\frac{d \mathbf{m}_{i}}{d t}=\frac{d \mathbf{e}_{r}}{d t} & =\frac{\partial \mathbf{e}_{r}}{\partial r_{i}} \dot{r}_{i}+\frac{\partial \mathbf{e}_{r}}{\partial \theta_{i}} \dot{\theta}_{i}+\frac{\partial \mathbf{e}_{r}}{\partial \phi_{i}} \dot{\phi}_{i}, \\
& =\dot{\theta}_{i} \mathbf{e}_{\theta}+\sin \theta_{i} \dot{\phi}_{i} \mathbf{e}_{\phi}, \\
\mathbf{m}_{i} \times \frac{\partial E}{\partial \mathbf{m}_{i}} & =\mathbf{e}_{r} \times\left(\frac{\partial E}{\partial \theta_{i}} \mathbf{e}_{\theta}+\frac{1}{\sin \theta_{i}} \frac{\partial E}{\partial \phi_{i}} \mathbf{e}_{\phi}\right), \\
= & \frac{\partial E}{\partial \theta_{i}} \mathbf{e}_{\phi}-\frac{1}{\sin \theta_{i}} \frac{\partial E}{\partial \phi_{i}} \mathbf{e}_{\theta}, \\
\mathbf{m}_{i} \times \frac{\partial E}{\partial \mathbf{m}_{i}} \times \mathbf{m}_{i} & =\left(\frac{\partial E}{\partial \theta_{i}} \mathbf{e}_{\phi}-\frac{1}{\sin \theta_{i}} \frac{\partial E}{\partial \phi_{i}} \mathbf{e}_{\theta}\right) \times \mathbf{e}_{r}, \\
& =\frac{\partial E}{\partial \theta_{i}} \mathbf{e}_{\theta}+\frac{1}{\sin \theta_{i}} \frac{\partial E}{\partial \phi_{i}} \mathbf{e}_{\phi} .
\end{aligned}
$$

By inserting Eqs. (3.49), (3.50), and (3.51) into Eq. (3.46), and equating the terms along $\mathbf{e}_{\theta}$ and $\mathbf{e}_{\phi}$ respectively, we obtain the following set of differential equations:

$$
\begin{aligned}
\frac{d \theta_{i}}{d t} & =\frac{g^{\prime}}{\sin \theta_{i}} \frac{\partial E}{\partial \phi_{i}}-h^{\prime} \frac{\partial E}{\partial \theta_{i}}, \\
\frac{d \phi_{i}}{d t} & =\frac{-g^{\prime}}{\sin \theta_{i}} \frac{\partial E}{\partial \theta_{i}}-\frac{h^{\prime}}{\sin ^{2} \theta_{i}} \frac{\partial E}{\partial \phi_{i}} .
\end{aligned}
$$

The next step consists in approximating the energy close to the saddle point $(\tilde{\theta}, \tilde{\phi})=\left(\tilde{\theta_{1}}, \ldots \tilde{\theta_{N}}, \tilde{\phi_{1}}, \ldots \tilde{\phi_{N}}\right)$ as a Taylor series truncated to the second order term,

$$
\begin{aligned}
E\left(\theta_{1}, \ldots \theta_{N}, \phi_{1}, \ldots \phi_{N}\right)= & E^{S}\left(\tilde{\theta_{1}}, \ldots \tilde{\theta_{N}}, \tilde{\phi_{1}}, \ldots \tilde{\phi_{N}}\right) \\
& +\frac{1}{2} \sum_{i, j}\left\{H_{\theta \theta_{i j}}^{S}\left(\theta_{i}-\tilde{\theta}_{i}\right)\left(\theta_{j}-\tilde{\theta}_{j}\right)+H_{\phi \phi_{i j}}^{S}\left(\phi_{i}-\tilde{\phi}_{i}\right)\left(\phi_{j}-\tilde{\phi}_{j}\right)\right. \\
& \left.+H_{\theta \phi_{i j}}^{S}\left(\theta_{i}-\tilde{\theta}_{i}\right)\left(\phi_{j}-\tilde{\phi}_{j}\right)+H_{\phi \theta_{i j}}^{S}\left(\phi_{i}-\tilde{\phi}_{i}\right)\left(\theta_{j}-\tilde{\theta}_{j}\right)\right\},
\end{aligned}
$$

where $E^{S}$ is the energy at $S$ and $H_{\theta \theta}^{S}, H_{\phi \phi}^{S}, H_{\theta \phi}^{S}, H_{\phi \theta}^{S}$ are the submatrices in the spherical Hessian defined in Eq. (3.45) and evaluated at $S$. Taking the derivative of Eq. (3.53) in $\theta_{i}$ and $\phi_{i}$ then yields

$$
\begin{aligned}
\frac{\partial E}{\partial \theta_{i}} & =\sum_{j}\left(H_{\theta \theta_{i j}}^{S}\left(\theta_{j}-\tilde{\theta}_{j}\right)+\frac{1}{2}\left(H_{\theta \phi_{i j}}^{S}+H_{\phi \theta_{j i}}^{S}\right)\left(\phi_{j}-\tilde{\phi}_{j}\right)\right), \\
\frac{\partial E}{\partial \phi_{i}} & =\sum_{j}\left(H_{\phi \phi_{i j}}^{S}\left(\phi_{j}-\tilde{\phi}_{j}\right)+\frac{1}{2}\left(H_{\theta \phi_{j i}}^{S}+H_{\phi \theta_{i j}}^{S}\right)\left(\theta_{j}-\tilde{\theta}_{j}\right)\right) .
\end{aligned}
$$


By noting that $H_{\phi \theta_{j i}}=H_{\theta \phi_{i j}}$ and $H_{\theta \phi_{j i}}=H_{\phi \theta_{i j}}$, the above equations reduce to

$$
\begin{aligned}
\frac{\partial E}{\partial \theta_{i}} & =\sum_{j}\left(H_{\theta \theta_{i j}}^{S}\left(\theta_{j}-\tilde{\theta}_{j}\right)+H_{\theta \phi_{i j}}^{S}\left(\phi_{j}-\tilde{\phi}_{j}\right)\right), \\
\frac{\partial E}{\partial \phi_{i}} & =\sum_{j}\left(H_{\phi \phi_{i j}}^{S}\left(\phi_{j}-\tilde{\phi}_{j}\right)+H_{\phi \theta_{i j}}^{S}\left(\theta_{j}-\tilde{\theta}_{j}\right)\right) .
\end{aligned}
$$

Finally, by inserting (3.55) into (3.52) evaluated at $S$, and defining small deviations from the saddle point as $\boldsymbol{\Theta}=\boldsymbol{\theta}-\tilde{\boldsymbol{\theta}}$ and $\boldsymbol{\Phi}=\boldsymbol{\phi}-\tilde{\boldsymbol{\phi}}$, we obtain the following system of equations linearized about the saddle point, which we write in matrix form,

$$
\left(\begin{array}{c}
\dot{\boldsymbol{\Theta}} \\
\dot{\boldsymbol{\Phi}}
\end{array}\right)=\mathcal{T}\left(\begin{array}{l}
\boldsymbol{\Theta} \\
\boldsymbol{\Phi}
\end{array}\right),
$$

in which the dot notation is a time derivative and

$$
\mathcal{T}=\left(\begin{array}{ll}
\mathcal{T}_{\theta \theta} & \mathcal{T}_{\theta \phi} \\
\mathcal{T}_{\phi \theta} & \mathcal{T}_{\phi \phi}
\end{array}\right)
$$

is the transition matrix of LLG, where

$$
\begin{aligned}
& \mathcal{T}_{\theta_{i} \theta_{j}}=g^{\prime} H_{\phi_{i} \theta_{j}}^{S}-h^{\prime} H_{\theta_{i} \theta_{j}}^{S}, \\
& \mathcal{T}_{\theta_{i} \phi_{j}}=\sin \tilde{\theta}_{j}\left(g^{\prime} H_{\phi_{i} \phi_{j}}^{S}-h^{\prime} H_{\theta_{i} \phi_{j}}^{S}\right), \\
& \mathcal{T}_{\phi_{i} \theta_{j}}=-\frac{1}{\sin \tilde{\theta}_{i}}\left(g^{\prime} H_{\theta_{i} \theta_{j}}^{S}+h^{\prime} H_{\phi_{i} \theta_{j}}^{S}\right), \\
& \mathcal{T}_{\phi_{i} \phi_{j}}=-\frac{\sin \tilde{\theta}_{j}}{\sin \tilde{\theta}_{i}}\left(g^{\prime} H_{\theta_{i} \phi_{j}}^{S}+h^{\prime} H_{\phi_{i} \phi_{j}}^{S}\right),
\end{aligned}
$$

define its submatrices. Similarly to the Hessian, the transition matrix is a $2 \mathrm{~N} \times 2 \mathrm{~N}$ matrix, but the number of positive eigenvalues it possesses equates to the order of the saddle point. The diagonalized transition matrix contains the eigenfrequencies of the system at the saddle point. In the most common configuration where the saddle point is of first order, the single positive eigenvalue of $\mathcal{T}$ is taken as the dynamical contribution to the attempt frequency, $\lambda_{+}$, and is interpreted as the growth rate the dynamically unstable deviation at the saddle [47]. In the case of a higher-order saddle point, it is not clear whether Langer's theory still applies. In [125], it was proposed that if several unstable modes are present at $S, \lambda_{+}$should be chosen as the biggest -i.e., most positive - eigenvalue of $\mathcal{T}$, thus giving the rate of the fastest growing instability. We can note that the transition matrix is not symmetric and can in principle admit complex eigenvalues and eigenvectors. Although we have only encountered real eigenvalues in 
this thesis, one example of a complex $\lambda_{+}$can be found in Ref. [125] in the case of the antiferromagnetic reversal of two ferromagnetic particles. In that case, $\lambda_{+}$should be taken as the largest real part amongst the eigenvalues. Rewriting Eq. (3.57) in the eigenbasis yields

$$
\left(\begin{array}{c}
\dot{\boldsymbol{\Theta}}^{\prime} \\
\dot{\boldsymbol{\Phi}}^{\prime}
\end{array}\right)=\mathcal{T}^{\prime}\left(\begin{array}{c}
\boldsymbol{\Theta}^{\prime} \\
\boldsymbol{\Phi}^{\prime}
\end{array}\right),
$$

where $\left(\boldsymbol{\Theta}^{\prime}, \boldsymbol{\Phi}^{\prime}\right)$ are the eigenbasis coordinates and $\mathcal{T}^{\prime}$ is the diagonalized transition matrix. In particular, the unstable mode evolves as

$$
\left(\Theta_{+}^{\prime}(t), \Phi_{+}^{\prime}(t)\right)=e^{\lambda_{+} t}\left(\Theta_{+}^{\prime}(t=0), \Phi_{+}^{\prime}(t=0)\right)
$$

If $\lambda_{+}$is real, the instability grows exponentially. On the other hand, if $\lambda_{+}$is a complex number, $\lambda_{+}=\sigma+i \omega$ with $\sigma, \omega \in \mathbb{R}, i^{2}=-1$, and $\sigma>0$, so

$$
\left(\Theta_{+}^{\prime}(t), \Phi_{+}^{\prime}(t)\right)=e^{\sigma t} e^{i \omega t}\left(\Theta_{+}^{\prime}(t=0), \Phi_{+}^{\prime}(t=0)\right)
$$

in which $e^{i \omega t}$ is a phasor. In that case, the time evolution of the instability is a sinusoid inside a growing exponential envelope. In all cases, the timescale of the transition is set by the damping and the characteristic precession time, $M_{s} /\left(J_{1} \gamma_{e}\right)$.

\subsection{The geodesic nudged elastic band method}

The nudged elastic band method (NEB, or NEBM) [55, 56] is a numerical scheme designed to compute paths of minimum energy through the (often complex) multidimensional energy landscape of a system connecting two energy minima. The NEB method has been most commonly used for transitions involving atomic rearrangements. The method consists in specifying a discrete representation of an initial path between the two minima corresponding to the initial and final states of the system for the transition of interest, and iteratively bringing the path to the nearest minimum energy path (MEP). Each discretization point along the path corresponds to a state of the complete system in the space of configurations, and is referred to as an image. Adjacent images are connected with springs that allow control over the resolution of the path and prevent the images from sliding down towards energy minima. A considerable advantage of the NEB method over similar methods of finding a MEP is that it does not require an initial guess of the reaction coordinate. Instead, it is naturally found as the path relaxes to a $\mathrm{MEP}$, at which point the tangent to the path provides a good estimate of the reaction coordinate. A $\mathrm{MEP}$ is a path of highest statistical weight and therefore corresponds to a particular mechanism of the considered transition. As was touched upon in the previous section, there can be one or several $\operatorname{MEP}(s)$, each of them associ- 


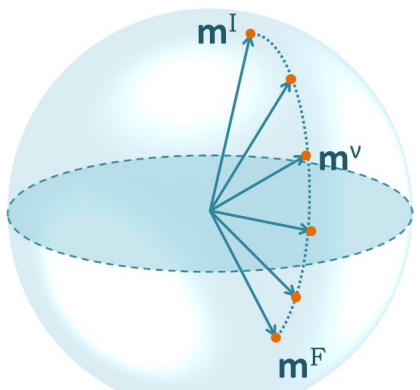

(a)

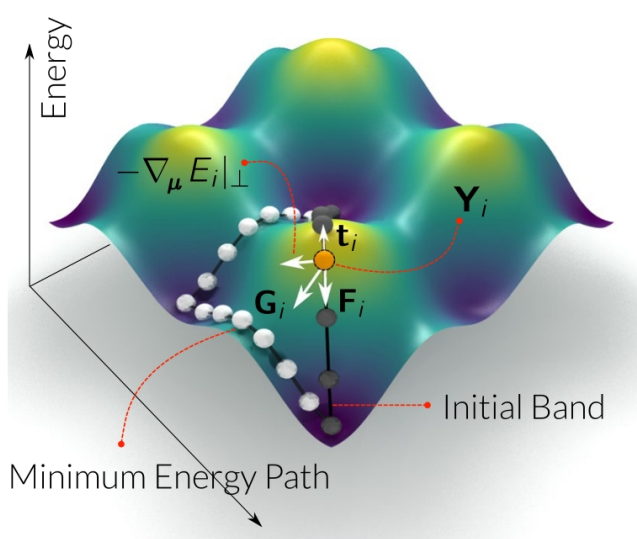

(b)

Figure 3.3: (a) Illustration of an elastic band in the GNEB method for a single spin system $\mathbf{m}$. The unit sphere $S^{2}$ corresponds to the space of configurations. The path between the initial state $\mathbf{m}^{I}$ and the final state $\mathbf{m}^{F}$ is discretized into a series of images $\left\{\mathbf{m}^{v}\right\}, v=1 \ldots Q$, marked as orange dots. (b) Illustration of the NEB method on a twodimensional energy landscape. The elastic band is a chain of images represented by small spheres. The initial path is shown in black. After minimization, the path relaxes to the MEP shown in white, which passes over two first-order saddle points separated by a local minimum (image source: [67]).

ated with a specific mechanism and transition rate. In particular, the NEB method can be combined with a scheme that allows a precise identification of the first-order saddle point along the path, such as the climbing image (CI) NEB, known as CI-NEB [58], and thus constitutes an important building block for a numerical implementation of reaction rate theory.

The geodesic NEB method (GNEB) is a variant of the NEB method adapted to magnetic systems which was developed by Bessarab et al. [59]. It solves potential convergence problems of the NEB method when applied to magnetic spin systems, by taking into account the curved geometry of the space of configurations. Its key features lie in the use of geodesics to calculate distances between images, and in the projection of the tangent to the path and of the force acting on the images onto the space tangent to the space of configurations. In what follows, we expose the principles of this method and provide details of its implementation.

\subsubsection{Projections onto the tangent space to the manifold}

The rest of this section is based on [59]. As we have assumed that the amplitude of the magnetic vectors remains fixed, each magnetic vector $\mathbf{m}_{i}$ thus lives on the unit sphere associated with site $i, S_{i}^{2}$, with its orientation specified by two coordinates (Fig. 3.3a). For instance, one may choose the spherical angles $(\theta, \phi)$. The configuration space 
of a system of $N$ spins is the $2 N$-dimensional Riemannian manifold $\mathscr{R}$ obtained from a direct product of the $N$ two-dimensional unit spheres $S_{i}^{2}$,

$$
\mathscr{R}=\prod_{i=1}^{N} S_{i}^{2} .
$$

In the GNEB scheme, the local tangent to path, as well as the total force acting on each image, are projected onto the tangent space to the manifold. We define the tangent space to the unit sphere, $S_{i}^{2}$, at $\mathbf{m}_{i}$, as the span of all vectors $\mathbf{x}$ normal to $\mathbf{m}_{i}$, that is,

$$
\mathcal{T}_{\mathbf{m}_{i}} S_{i}^{2}=\left\{\mathbf{x}: \mathbf{x} \cdot \mathbf{m}_{i}=0\right\}
$$

The tangent space to the $2 N$-dimensional Riemannian manifold $\mathscr{R}$ is simply defined as the product of the tangent spaces to each sphere,

$$
\mathcal{T}_{\mathbf{m}_{i}} \mathscr{R}=\prod_{i=1}^{N} \mathcal{T}_{\mathbf{m}_{i}} S_{i}^{2} .
$$

The projection of a three-dimensional Cartesian vector $\mathbf{a}_{i}$ onto $\mathcal{T}_{\mathbf{m}_{i}} \mathscr{R}$ is carried out by substracting its component along $\mathbf{m}_{i}$,

$$
\mathbf{a}_{i, \mathcal{T}}=\mathbf{a}_{i}-\left(\mathbf{a}_{i} \cdot \mathbf{m}_{i}\right) \mathbf{m}_{i}
$$

In this section, we choose the following convention: while all vectors remain in bold, local three dimensional vectors a will be represented by a lowercase letter, while $3 \mathrm{~N}$ dimensional vectors are written as the corresponding uppercase letter as $\mathbf{A}=\left(\mathbf{a}_{1}, \mathbf{a}_{2}, \ldots \mathbf{a}_{N}\right)$. We can now define the projection of a $3 N$-dimensional vector $\mathbf{A}$ onto the tangent space to the $\mathscr{R}$-manifold as

$$
\mathcal{P}_{\mathcal{T}} \mathbf{A}=\left(\mathbf{a}_{1, \mathcal{T}}, \mathbf{a}_{2, \mathcal{T}}, \ldots \mathbf{a}_{N, \mathcal{T}}\right)
$$

\subsubsection{The GNEB method}

We construct a chain of $Q$ images, $\left[\mathbf{M}^{1}, \mathbf{M}^{2} \ldots \mathbf{M}^{Q}\right]$ (Fig. 3.3a), where each image $\mathbf{M}^{v}=\left(\mathbf{m}_{1}^{v}, \mathbf{m}_{2}^{v} \ldots \mathbf{m}_{N}^{v}\right)$, with $v=1 \ldots Q$, is a $3 N$-dimensional Cartesian vector. The chain of images constitutes the elastic band. The first and last images, $\mathbf{M}^{1}=\mathbf{M}^{I}$ and $\mathbf{M}^{Q}=$ $\mathbf{M}^{F}$, are chosen as energy minima corresponding to the initial and final states of the transition, and remain fixed. The remaining images are iteratively brought to a MEP through minimization of the total GNEB force acting on them (Fig. 3.3b). That force is the sum of the negative energy gradient perpendicular to the path, i.e., the true force, and a spring force acting along the path. This requires an estimation of the tangent to 
the path at each image.

The tangent to the path at image $v, \boldsymbol{\tau}^{v}$, is evaluated as detailed in Sec. 3.4.3, and projected onto the tangent space according to Eq. (3.66),

$$
\boldsymbol{\tau}_{\mathcal{T}}^{v}=\frac{\mathcal{P}_{\mathcal{T}} \tau^{v}}{\left|\mathcal{P}_{\mathcal{T}} \tau^{v}\right|}
$$

The true force acting on image $v$ originates from the transverse (perpendicular) component of the negative gradient, and is obtained by subtracting the component of the gradient along the tangent to the path,

$$
\nabla E\left(\mathbf{M}^{v}\right)_{\perp}=\nabla E\left(\mathbf{M}^{v}\right)-\left(\nabla E\left(\mathbf{M}^{v} \cdot \boldsymbol{\tau}_{\mathcal{T}}^{v}\right) \boldsymbol{\tau}_{\mathcal{T}}^{v}\right.
$$

The spring force, on the other hand, only has a component along the path tangent and its magnitude depends on the geodesic distances between adjacent images, that is,

$$
\mathbf{F}_{S \|}^{v}=\kappa^{v}\left[L\left(\mathbf{M}^{v+1}, \mathbf{M}^{v}\right)-L\left(\mathbf{M}^{v}, \mathbf{M}^{v-1}\right)\right] \boldsymbol{\tau}_{\mathcal{T}}^{v}
$$

In the previous expression, $\kappa^{v}$ is the spring constant at image $v$, which can be chosen to be constant at all images, or it may be adjusted to gain resolution in critical areas, typically around a saddle point. We give details on the variable spring scheme [58] later in this section. $L\left(\mathbf{M}^{v}, \mathbf{M}^{\mu}\right)$ is the geodesic distance between two images $v$ and $\mu$ on the $\mathscr{R}$-manifold. It is defined as

$$
L\left(\mathbf{M}^{v}, \mathbf{M}^{\mu}\right)=\sqrt{\left(l_{1}^{v, \mu}\right)^{2}+\left(l_{2}^{v, \mu}\right)^{2}+\ldots+\left(l_{N}^{v, \mu}\right)^{2}},
$$

where the $l_{i}^{v, \mu}$ is the local geodesic distance between $v$ and $\mu$ on the unit sphere $S_{i}^{2}$, which we evaluate as

$$
l_{i}^{\nu, \mu}=2 \arcsin \sqrt{\frac{1-\mathbf{m}^{\nu} \cdot \mathbf{m}^{\mu}}{2}} .
$$

This projection of the true force and the spring force using the tangent to the path is referred to as "nudging". Finally, the total GNEB force acting on image $v$ needs to be projected onto the tangent space. In the end, the force applied to the images is expressed as

$$
\mathbf{F}_{\mathrm{GNEB}}^{v}=\mathcal{P}_{\mathcal{T}}\left(-\nabla E\left(\mathbf{M}^{v}\right)_{\perp}+\mathbf{F}_{S \|}^{v}\right)
$$

The projection of the tangent to the path onto the tangent space to the curved manifold allows a decoupling of the spring force and the true force, so that they do not interfere with each other. If this procedure is not carried out, the control over the distribution of images is lost and the scheme becomes sensitive to the value of the spring constant [59]. 
The path will usually converge to the MEP closest to the initial path. If several transition mechanisms exist, several runs of the GNEB scheme with different initializations of the path must to be carried out, until all possible MEPs are identified. An example run of the GNEB scheme is shown in Fig. 3.4 for a single spin reversal with a hard-axis along $z$. The path is initialized out of plane with some random noise added to the initial distribution of the images. The scheme iteratively brings the path to the $x y$-plane and uniformly redistributes the images.

\subsubsection{Evaluation of the tangent to the path}

An appropriate definition of the tangent to the path at image $v, \tau^{v}$, is one key aspect to the stability of the method. We used the definition from [59],

$$
\boldsymbol{\tau}^{v}= \begin{cases}\boldsymbol{\tau}_{+}^{v}, & \text { if } E^{v+1}>E^{v}>E^{v-1} \\ \boldsymbol{\tau}_{-}^{v}, & \text { if } E^{v+1}<E^{v}<E^{v-1}\end{cases}
$$

where $\boldsymbol{\tau}_{+}^{v}=\mathbf{M}^{v+1}-\mathbf{M}^{v}$ and $\boldsymbol{\tau}_{-}^{v}=\mathbf{M}^{v}-\mathbf{M}^{v-1}$, and $E^{v}=E\left(\mathbf{M}^{v}\right)$. If the image is close to a maximum or a minimum in the energy,

$$
\boldsymbol{\tau}^{v}= \begin{cases}\boldsymbol{\tau}_{+}^{v} \Delta E_{\max }^{v}+\boldsymbol{\tau}_{-}^{v} \Delta E_{\min }^{v} & \text { if } E^{v+1}>E^{v-1}, \\ \boldsymbol{\tau}_{+}^{v} \Delta E_{\min }^{v}+\tau_{-}^{v} \Delta E_{\max }^{v} & \text { if } E^{v+1}<E^{v-1}\end{cases}
$$

where $\Delta E_{\max }^{v}=\max \left(\left|E^{v+1}-E^{v}\right|,\left|E^{v-1}-E^{v}\right|\right)$ and $\Delta E_{\min }^{v}=\min \left(\left|E^{v+1}-E^{v}\right|,\left|E^{v-1}-E^{v}\right|\right)$. Eq. (3.74) provides a smooth transition between the forward and backward definitions, $\boldsymbol{\tau}_{+}^{v}$ and $\boldsymbol{\tau}_{-}^{v}$. The above definition of the tangent to the path according to Eqs. (3.73) and (3.74) enhances the stability of the method by preventing the formation of kinks in the path [129].

\subsubsection{Initialization of the path along geodesics}

The initialization of the GNEB scheme requires an initial path between the initial states to be generated. If a good guess cannot be made, a solution is to evenly distribute the images along the geodesic path connecting the first and last image. We have followed the procedure described in [59], which makes use of Rodrigues' rotation formula to generate the orientation of the local magnetic vector $\mathbf{m}_{i}^{v}$ at image $v$ as

$$
\mathbf{m}_{i}^{v}=\mathbf{m}_{i}^{F} \cos \omega_{i}^{v}+\left(\mathbf{k}_{i} \times \mathbf{m}_{i}^{I}\right) \sin \omega_{i}^{v},
$$


where $\omega_{i}^{v}$ is the rotation angle and $\mathbf{k}_{i}$ is the rotation vector. The angle of rotation $\omega_{i}^{v}$ is a fraction of the total angle $\omega_{i}$ between $\mathbf{m}_{i}^{I}$ and $\mathbf{m}_{i}^{F}$,

$$
\omega_{i}^{v}=\frac{(v-1) \omega_{i}}{Q-1}
$$

and $\omega_{i}=\arccos \left(\mathbf{m}_{i}^{I} \cdot \mathbf{m}_{i}^{F}\right)$. Finally, the normalized axis of rotation is defined by

$$
\mathbf{k}_{i}=\frac{\mathbf{m}_{i}^{I} \times \mathbf{m}_{i}^{F}}{\left|\mathbf{m}_{i}^{I} \times \mathbf{m}_{i}^{F}\right|}
$$

\subsubsection{Search for the first-order saddle point: the CI-GNEB scheme}

If one is only looking to estimate the energy barrier, this can in principle be done by interpolating the energy along the relaxed path. However, the use of Langer's theory to calculate a rate prefactor, like any modern form of reaction rate theory, requires a precise identification of the saddle point along the MEP. This can be achieved by a climbing image (CI) scheme [58]. After the regular GNEB scheme has relaxed down to a certain tolerance on the GNEB force, the highest energy image becomes a climbing image. The spring force on this particular image is dropped, and instead the CI is made to move uphill in energy along the reaction coordinate, which is defined by the tangent to the path, and downhill in energy along all other degrees of freedom. For that purpose, the force acting on the CI takes the form,

$$
\mathbf{F}_{G N E B}^{v_{C I}}=\mathcal{P}_{\mathcal{T}}\left(-\nabla E\left(\mathbf{M}^{v_{C I}}\right)+2\left(\nabla E\left(\mathbf{M}^{v_{C I}}\right) \cdot \tau_{\mathcal{T}}^{v_{C I}}\right) \tau_{\mathcal{T}}^{v_{C I}}\right)
$$

By minimizing the force acting on it, the CI will, in most cases, relax to the highest energy first-order saddle point on the path. If an energy interpolation reveals the existence of multiple saddle points along the path, two or more climbing images may be specified. During the CI-GNEB procedure, the rest of the images still need to be displaced according to Eq. (3.72). Like previously, the procedure ends once the force on all images is relaxed below a set tolerance. We can verify that we have indeed found a first-order saddle point by checking that the Hessian evaluated at that point possesses only a single negative eigenvalue.

\subsubsection{Variable spring constants}

The spring force in Eq. (3.69) that controls the distribution of images along the path may be adjusted as to increase the resolution of the path in certain areas of interest. Typically, a better resolution around the saddle point will increase the accuracy of the path tangent and provide a better estimate of the reaction coordinate for the climbing 

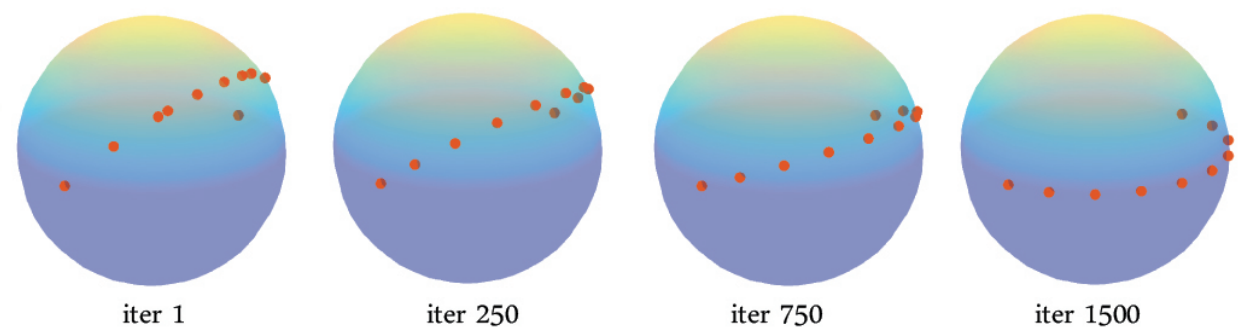

Figure 3.4: Illustration of the GNEB scheme for a single spin $\mathbf{m}$ undergoing a magnetization reversal from $m_{x}=-1$ to $m_{x}=1$ with easy-axis anisotropy along $\mathbf{e}_{x}$, and hard-axis anisotropy along $\mathbf{e}_{z}$. The initialization is done out-of-plane with a random noise added to the distribution of the images and the scheme iteratively brings the images in-plane and redistributes them uniformly along the path.

image to follow. Because the true force and the spring force are decoupled, the use of different springs between different pairs of images does not affect the convergence to the MEP, as long as the number of images is sufficient. We have used the scheme proposed in [58] which results in lower energy images being connected by weaker springs, as follows,

$$
\kappa^{v}= \begin{cases}\kappa_{\max }-\Delta \kappa\left(\frac{E_{\max }-E_{\max }^{v}}{E_{\max }-E^{\mathrm{ref}}}\right) & \text { if } E_{\max }^{v}>E^{\mathrm{ref}}, \\ \kappa_{\max }-\Delta \kappa & \text { if } E_{\max }^{v} \leq E^{\mathrm{ref}} .\end{cases}
$$

$\kappa^{v}$ is the spring constant associated with spring $v$ (where $v=2 \ldots Q$ ) connecting images $v-1$ and $v, E_{\max }^{v}=\max \left(E^{v-1}, E^{v}\right)$ is the highest energy of the two images connected by spring $v$, and $E_{\max }$ is the maximum value of $E^{v}$ amongst all images. $E^{\text {ref }}$ is a reference value for the energy, which we set as the highest energy of the initial and final states, $E^{\text {ref }}=\max \left(E^{I}, E^{F}\right)$ as suggested in [58]. This results in a maximum value of the spring constant of $\kappa_{\max }$ between highest energy images, and a minimum value of $\kappa_{\max }-\Delta \kappa$ for images with energy $E^{\text {ref }}$ or lower.

We have found that in certain cases, possibly when two MEPs exist close to each other, using variable springs might even lead to a different MEP with a different saddle point, compared to the same GNEB run with constant springs. This occurs, for instance, when relaxing the paths to collapse of chiral skyrmions. While the regular GNEB scheme with constant springs along the elastic band relaxes the path where the skyrmion core remains centred on a lattice site (mechanism $n^{0} 1$ ), the addition of variable springs when the path is initialized as geodesics according to Sec. 3.4.4 leads to a separate mechanism being relaxed, in which the skyrmion core shifts to an interstitial position (mechanism $\mathrm{n}^{\circ} 2$ ). These two mechanisms will be described in more details in Sec. 4.3 . 


\subsection{Implementation of Langer's theory on an atomistic spin lattice}

\subsubsection{Atomistic simulations and search for local minima}

The following methods were implemented by the author as an atomistic code in $\mathrm{C}++$ language over the course of this thesis. The calculations were carried out in Cartesian coordinates, with the exception of the rate prefactor. All quantities are dimensionless, in units of the isotropic exchange coupling constant between first neighbours, $J_{1}=J_{\text {ex }}$, as defined in Sec. 2.1.4. The atomistic Hamiltonian on the two-dimensional square lattice is calculated from Eq. (2.1). The reduced local effective field acting on spin $\mathbf{m}_{i}$ at site $i$ is

$$
\mathbf{b}_{\text {eff }}^{i}=-\frac{1}{J_{1}} \frac{\partial E}{\partial \mathbf{m}_{i}} .
$$

We relax local energy minima by the simple iterative energy minimization procedure described in Sec. 11.2 of Ref [130]. The system is initialized close to a local energy minimum of interest. For instance, we initialize a central square of reversed spin on a ferromagnetic background in order to relax a skyrmion. After that, a normalized effective field at spin $i$,

$$
\hat{\mathbf{b}}_{\text {eff }}^{i}=\mathbf{b}_{\text {eff }}^{i} /\left|\mathbf{b}_{\text {eff }}^{i}\right|,
$$

is calculated according to Eq. (3.80), and $\mathbf{m}_{i}$ is aligned onto the local field by simply setting $\mathbf{m}_{i}=\hat{\mathbf{b}}_{\text {eff. }}^{i}$ We then move on to another spin and proceed analogously. The iterations end when the largest component of $\mathbf{m}_{i} \times \hat{\mathbf{b}}_{\text {eff }}^{i}$ falls below a set tolerance $\epsilon$. In general we set $\epsilon \in\left[10^{-8}-10^{-15}\right]$. This method converges to the minimum that is closest to the initialization state, and is therefore well adapted to the search for metastable states. While it may fail for very simple cases (eg: a single macrospin), it works well for $N$-spin systems $(N>1)$ and yields the same results as the integration of the LLG equation (Eq. (3.46)) at zero temperature.

\subsubsection{Implementation of GNEB}

\section{Overview of the GNEB algorithm}

We give an overview of the GNEB and CI-GNEB algorithm:

- The first and last images, $\mathbf{M}^{I}$ and $\mathbf{M}^{F}$, are initialized as energy minima. These are usually previously generated via the method described in Sec. 3.5.1, or they may also be relaxed through integration of the LLG equation (3.46), or via the GNEB scheme [59]. 
- An initial path $\left[\mathbf{M}^{2}, \mathbf{M}^{3} \ldots \mathbf{M}^{Q-1}\right]$ between $\mathbf{M}^{I}$ and $\mathbf{M}^{F}$ is generated, either along geodesics as described in Sec. 3.4.4, or it is manually implemented as part of an initial guess.

- The GNEB scheme begins and runs until all Cartesian components of the local force on each spin at each image falls below a set tolerance $F_{\text {tol }}$.

- The highest energy image is selected as the climbing image and the CI-GNEB scheme runs until the force falls below the set tolerance.

- The energy between the images is interpolated by a piecewise cubic interpolation, as described in [59].

\section{Choice of solver}

Although one could choose to integrate a precessionless version of the LLG equation at each timestep, in our case the force minimization during the (CI)-GNEB procedure was done as suggested in [59], by simply integrating Newton's equations for the motion of a fictitious point-mass on the unit sphere (Fig. 3.4), where the total force is the GNEB force, i.e.,

$$
\mathbf{F}_{\mathrm{GNEB}}^{v}=m \Gamma^{v}
$$

In the previous expression, $m$ is an arbitrary mass that controls the inertia of the images, which we set to $m=1$, and $\Gamma^{v}=d^{2} \mathbf{M}^{v} / d t^{2}=\left(\gamma_{1}^{v}, \gamma_{2}^{v} \ldots \gamma_{N}^{v}\right)$ is the acceleration of image $v$. The value of the spring constant $\kappa_{\max }$ is not critical in GNEB [59], but large values tend to make the path longer in certain cases, so we set $\kappa_{\max }=1$ and $\Delta \kappa=0.1$. Depending on the problem at hand, we typically use $Q=8-15$ images. The tolerance on each Cartesian component of the GNEB force at each image and each spin is set around $F_{\text {tol }}=10^{-10}-10^{-12}$. Although Ref. [59] recommends the use of spherical coordinates so that the constraints on the magnetic vectors are automatically fulfilled, in practice, because the spherical coordinate system has singularities at the poles of the sphere (i.e., the azimuth $\phi$ is not defined for $\theta=0$ or $\pi$ ), a special treatment is required in these areas. Therefore, we use Cartesian coordinates instead, and re-normalize the magnetic vectors at each iteration. We use the velocity Verlet integrator [131], whose global error is of order 2 , with an integration step $\Delta t=0.01$.

The convergence is aided by the velocity projection optimization (VPO) procedure [59]. It consists in damping the velocity at each time-step by keeping only its component along the force. That is, unless the velocity is pointing in a direction opposite to that of the force, in which case it is set to zero. A similar method was used for NEB 
calculations in unconstrained Euclidian space [56]. We define the velocity at image $v$,

$$
\mathbf{V}^{v}=\frac{d \mathbf{M}^{v}}{d t}=\left(\mathbf{v}_{1}^{v}, \mathbf{v}_{2}^{v} \ldots \mathbf{v}_{N}^{v}\right)
$$

Let

$$
\mathscr{F}=\left[\mathrm{F}_{\mathrm{GNEB}}^{1}, \mathrm{~F}_{\mathrm{GNEB}}^{2} \ldots \mathrm{F}_{\mathrm{GNEB}}^{\mathrm{Q}}\right]
$$

be a $3 N \times Q$-dimensional vector of the force, and

$$
\mathscr{V}=\left[\mathbf{V}^{1}, \mathbf{V}^{2} \ldots \mathbf{V}^{Q}\right]
$$

a $3 N \times Q$-dimensional vector of the velocity. For convenience, we define $a=\mathscr{V} \cdot \mathscr{F} / Q$ and $b=\mathscr{F} \cdot \mathscr{F} / Q$ as vector projections in $3 N \times Q$-dimensional space. At each timestep, the velocity is revised according to [59, 132],

$$
\mathscr{V}= \begin{cases}a \mathscr{F} / b & \text { if } a>0, \\ 0 & \text { if } a \leq 0,\end{cases}
$$

and used in the next iteration. As a result, if the force keeps pointing in the same direction, the system accelerates and approaches the minimum faster. Then, if the system overshoots the minimum, the velocity is set to zero.

\section{Force minimization algorithm}

The aim of the GNEB method is to relax the initial path onto a MEP by minimizing the GNEB force acting on the images. One proceeds as follows. First, the scheme is initialized as described below. At each image $v=1 \ldots Q$ :

- the energy $E^{v}=E\left(\mathbf{M}^{v}\right)$ is calculated;

- the tangent to the path is calculated as described in Sec. 3.4.3, and projected onto the tangent space according to Eq. (3.67);

- the total force $\mathrm{F}_{\mathrm{GNEB}}^{v}$ is calculated and projected onto the tangent space according to Eq. (3.72);

- the acceleration is initialized as $\Gamma^{v}=\mathrm{F}_{\mathrm{GNEB}}^{v} / m$;

- the velocity $\mathbf{V}^{v}$ is set to zero.

This completes the initialization. After that, the following procedure is iterated until the set tolerance on the components of the GNEB force has been reached: 
- the position of each image $\mathbf{M}^{v}$ is updated according to

$$
\mathbf{M}^{v}(t+d t)=\mathbf{M}^{v}(t)+\mathbf{V}^{v}(t) d t+\frac{1}{2} \Gamma^{v}(t) d t^{2},
$$

and renormalized;

- then, at each image:

- the new energy $E^{v}(t+d t)=E\left(\mathbf{M}^{v}(t+d t)\right)$ is calculated;

- the new projected tangent to the path $\boldsymbol{\tau}_{\mathcal{T}}^{v}(t+d t)$ is calculated;

- the new projected GNEB force $\mathbf{F}_{\mathrm{GNEB}}^{v}(t+d t)$ is calculated and its local components are compared to the set tolerance;

- the acceleration is updated as $\Gamma^{v}(t+d t)=\mathbf{F}_{\mathrm{GNEB}}^{v}(t+d t) / m$;

- the velocity is updated according to

$$
\mathbf{V}^{v}(t+d t)=\mathbf{V}^{v}(t)+\frac{1}{2}\left(\Gamma^{v}(t+d t)+\Gamma^{v}(t)\right) d t
$$

- the velocity $\mathbf{V}^{v}(t+d t)$ is revised at each image according to the VPO procedure.

\subsubsection{Computation of the rate prefactor}

Once the CI-GNEB scheme has relaxed to a low tolerance, we have in principle identified the configuration of the (highest) first-order saddle point $S$ along the reaction coordinate to high accuracy. This gives us a straightforward value for the internal energy barrier as $\Delta E=E_{S}-E_{A}$. One can now compute the pre-exponential factor in Langer's theory, as given in Eq. (3.31). Since the space of configurations is $2 \mathrm{~N}$-dimensional, we found the use of spherical coordinates to be a better choice at this point in the computation. We thus begin by converting the positions of states $A$ and $S$ into spherical angles $(\theta, \phi)$. If at least one spin $\mathbf{m}_{i}$ lies in the vicinity of the poles of the sphere, i.e., $\left|\sin \theta_{i}\right|<\varepsilon$, where $\varepsilon$ is chosen as $\varepsilon \approx 10^{-8}$, the whole system is rotated about the $y$-axis by an arbitrary angle, until there are no spins close to the poles. This includes a rotation of the external magnetic field, the anisotropy axis, and the Dzyaloshinskii vector, as well as all the magnetic vectors.

After that, we can construct the submatrices in the Hessian matrix evaluated at $A$ and $S$, as previously defined in Eqs. (3.44) and (3.45). In order to keep numerical noise to a minimum, these were implemented with analytical expressions for the second derivatives of the different terms of the Hamiltonian. The Hessian is then diagonalized using the eigensystems functions for real symmetric matrices from the GNU 
scientific library [133]. The library uses the symmetric bidiagonalization and QR reduction method. The $2 N$ eigenvalues $\left\{\lambda_{i}\right\}$ are then sorted by increasing value, and we also store the corresponding eigenvectors $\left\{\chi_{i}\right\}$. It is a good idea to check that there is indeed only one negative eigenvalue at $S$, a sign that we have found a first-order saddle point.

Next, the determination of $\lambda_{+}$is carried out. We use the submatrices of the Hessian to construct the transition matrix $\mathcal{T}$, as defined in Eqs. (3.57) and (3.58). To extract $\lambda_{+}$, we can take advantage of the fact that, as long as longe-range dipolar couplings are not included in the Hamiltonian, $\mathcal{T}$ is a sparse matrix. For that purpose, we use the Spectra library [134] built on top of the open source linear algebra library Eigen [135].

Finally, one has to test for possible zero-eigenvalue(s) in the Hessian. If some eigenvalues are close to 0 , their contribution to the prefactor needs to be handled separately, as described in Sec. 3.2.2. Otherwise, the ratio of eigenvalues can be calculated, and we obtain the rate prefactor of the transition.

\subsubsection{Numerical tests}

\section{One dimensional domain wall profile}

We first of all relaxed a one dimensional domain wall, and checked the profile against the theoretical expression (Eq. 2.32). Both the atomistic and theoretical profiles are plotted in Fig. 3.5a and show excellent agreement.

\section{Skyrmion profile}

The relaxed skyrmion profile was compared with the output of the micromagnetic simulation program MuMax3 [136]. We give the following reduced parameters, as defined in Sec. 2.1.4 $(d, k, b)=(0.36,0.4,0.0)$, which allow the existence of small isolated skyrmions at zero applied field [102]. We simulated a square lattice of $50 \times 50$ spins with open (non periodic) boundary conditions. The profile is plotted in Fig. $3.5 \mathrm{~b}$ and agrees well with the output of MuMax3. Here, we used a continuous definition of the skyrmion radius, as given in Ref. [102], which is the intersection of the largest gradient of the interpolated skyrmion profile with the $\theta=0$ axis. The largest gradient line is shown in blue in Fig. $3.5 \mathrm{~b}$ and intersects the horizontal axis at $r_{\mathrm{sk}}=5.83 a$.

\section{Test for the Hessian implementation}

A straightforward way to test for the correct analytical implementation of the Hessian is to compare it with a numerical implementation using finite differences. Let $d \theta$ 


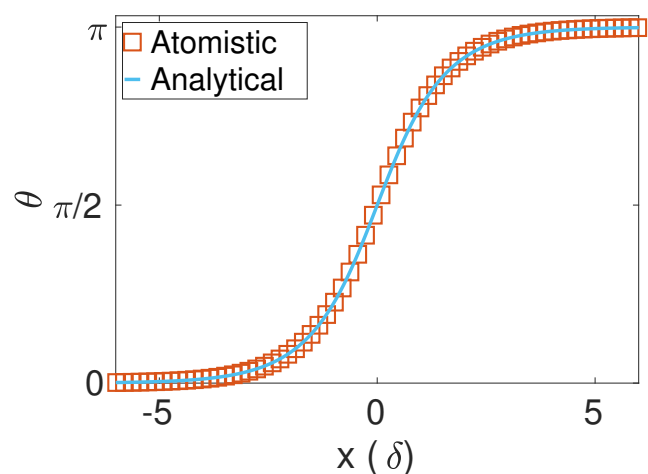

(a)

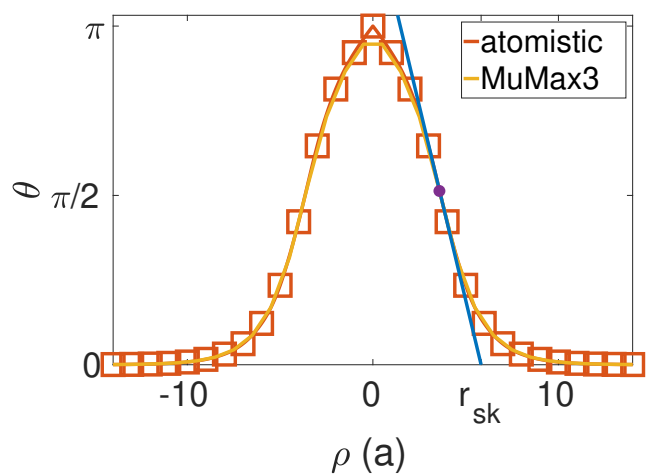

(b)

Figure 3.5: (a) Analytical micromagnetic and numerical atomistic $\pi$-domain wall profiles as a function of the spatial displacement from the wall centre, expressed in units of the characteristic wall length $\delta$. The analytical profile was previously derived in Sec. 2.2.1 and its expression was given in Eq. (2.32). (b) Comparison of the relaxed skyrmion profiles at zero temperature and reduced parameters $(d, k, b)=(0.36,0.4,0.0)$, as computed by our atomistic implementation and the micromagnetic program $\mathrm{Mu}-$ Max3. The skyrmion radius is given by the intersection of the largest gradient line of the interpolated profile (shown in blue) with the horizontal $\theta=0$ axis.

and $d \phi$ be small angular variations about a given state $(\theta, \phi)$, with energy $E(\theta, \phi)=E_{0}$. We set, for instance, $d \theta=d \phi=0.001$. We give the expressions for the submatrices in the Hessian, as defined in Eq. (3.45), using finite differences,

$$
\begin{gathered}
H_{\theta_{i} \theta_{j}} \approx \begin{cases}\frac{E\left(\theta_{i}+d \theta, \theta_{j}+d \theta\right)-E\left(\theta_{i}+d \theta, \theta_{j}-d \theta\right)-E\left(\theta_{i}-d \theta, \theta_{j}+d \theta\right)+E\left(\theta_{i}-d \theta, \theta_{j}-d \theta\right)}{4 d \theta^{2}} & \text { if } i \neq j, \\
\frac{E\left(\theta_{i}+d \theta\right)-2 E_{0}+E\left(\theta_{i}-d \theta\right)}{d \theta^{2}} & \text { if } i=j,\end{cases} \\
H_{\theta_{i} \phi_{j}} \approx \frac{E\left(\theta_{i}+d \theta, \phi_{j}+d \phi\right)-E\left(\theta_{i}+d \theta, \phi_{j}-d \phi\right)-E\left(\theta_{i}-d \theta, \phi_{j}+d \phi\right)+E\left(\theta_{i}-d \theta, \phi_{j}-d \phi\right)}{4 d \theta \sin \theta_{j} d \phi}, \\
H_{\phi_{i} \theta_{j}} \approx \frac{E\left(\theta_{j}+d \theta, \phi_{i}+d \phi\right)-E\left(\theta_{j}+d \theta, \phi_{i}-d \phi\right)-E\left(\theta_{j}-d \theta, \phi_{i}+d \phi\right)+E\left(\theta_{j}-d \theta, \phi_{i}-d \phi\right)}{4 d \theta \sin \theta_{i} d \phi} \\
H_{\phi_{i} \phi_{j}} \approx\left\{\begin{array}{l}
\frac{E\left(\phi_{i}+d \phi\right)-2 E_{0}+E\left(\phi_{i}-d \phi\right)}{d \phi^{2} \sin \theta_{i} \sin \theta_{j}} \\
4 d \phi^{2} \sin \theta_{i} \sin \theta_{j}
\end{array}\right. \\
\begin{array}{ll}
\frac{E\left(\phi_{i}+d \phi, \phi_{j}+d \phi\right)-E\left(\phi_{i}+d \phi, \phi_{j}-d \phi\right)-E\left(\phi_{j}-d \phi, \phi_{j}+d \phi\right)+E\left(\phi_{i}-d \phi, \phi_{j}-d \phi\right)}{2} \\
\text { if } i \neq j,
\end{array}
\end{gathered}
$$


Note that notations of the type $E\left(\theta_{i}+d \theta, \phi_{j}+d \phi\right)$ are a compact way to write

$$
E\left(\theta_{1}, \theta_{2}, \ldots, \theta_{i}+d \theta, \ldots, \theta_{N}, \phi_{1}, \phi_{2}, \ldots, \phi_{j}+d \phi, \ldots, \phi_{N}\right)
$$

The above expressions are not sufficiently accurate to compute a prefactor, and one should in principle go to higher orders, as is for instance the case in Ref. [62]. However, they are sufficient to provide a good mean of verification for the analytical Hessian implementation.

\section{Magnetization reversal in a perpendicular applied field}

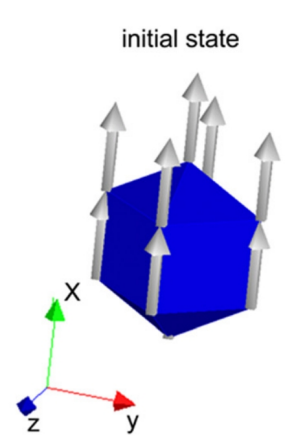

(a)

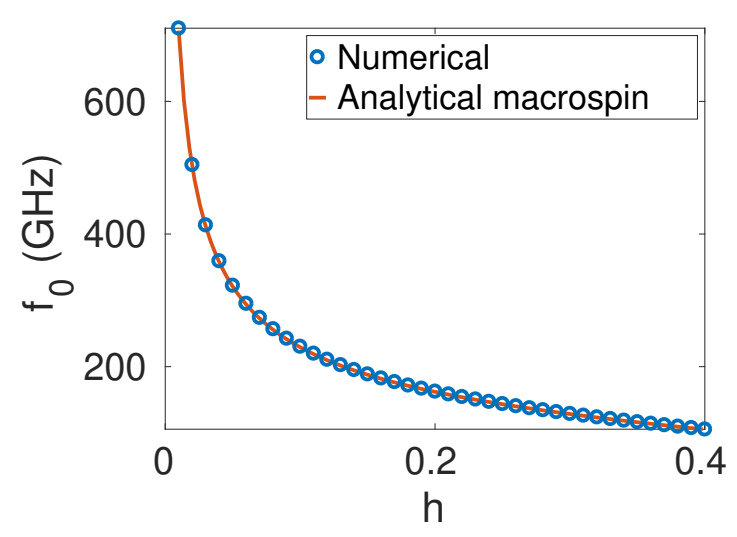

(b)

Figure 3.6: (a) Initial state and saddle point of the magnetization reversal in a perpendicular field applied along $y$ (image source: [62]). (b) Attempt frequency of coherent magnetization reversal for different values of the reduced perpendicular applied field $h$. The analytical expression is derived for a single macrospin using Langer's theory. The numerical expression is calculated for a lattice of $4 \times 4$ spins.

Next, the complete implementation of Langer's theory was tested against a simple analytical case, which we reproduced from Ref. [62]. We looked at magnetization reversal of a small magnetic square in two dimensions. The Hamiltonian contains an easy-axis anisotropy term and an external magnetic field perpendicular to the easy axis. Analytically, the system can be approximated by a single macrospin, as long as the size of the system remains small, so that the contribution from collective modes leads to a negligible error in the attempt frequency. We write the Hamiltonian of the single macrospin in spherical coordinates,

$$
E=-K\left(\sin ^{2} \theta \cos ^{2} \phi+2 h \sin \theta \sin \phi\right)
$$

where

$$
h=\frac{B M_{s}}{2 K}
$$


is the reduced perpendicular field. Note that we are using atomistic quantities as defined in Sec. 2.1.4, in contrast to Refs. [62, 137] where the quantities are micromagnetic. The degeneracy in the angle $\theta$ is lifted by the perpendicular field, so in this case $\theta=\pi / 2=\theta_{A}=\theta_{S}=\theta_{B}$. The potential is symmetric, with two minima of the energy at $\phi_{A}=\arcsin (h)$ and $\phi_{B}=\pi-\arcsin (h)$. The saddle point is found at $\phi_{S}=\pi / 2$ (Fig. 3.6a). By applying Langer's theory, the attempt frequency for the magnetization reversal was derived in Ref. [137] and is expressed as

$$
f_{0}=\frac{1}{2 \pi} \lambda_{+} \Omega_{0}=\frac{1}{2 \pi}\left[\frac{\gamma_{e} K}{M_{s}\left(1+\alpha^{2}\right)}\left(\alpha(1-2 h)+\sqrt{\alpha^{2}-4 h(h-1)}\right)\right] \sqrt{1+\frac{1}{h}} .
$$

In the previous expression, the gyromagnetic factor for the free electron, $\gamma_{e}$, and the damping factor, $\alpha$, are as defined in Sec. 3.3.2. We used the following parameters: $N=4 \times 4, a=0.1 \mathrm{~nm}, \alpha=0.5, \mathscr{K}=1 \mathrm{MJ} / \mathrm{m}^{3}, \mathscr{M}_{s}=0.5 \mu_{0}^{-1} \mathrm{~A} / \mathrm{m}$, and $\gamma_{e}=1.76 \times 10^{11}$ $\operatorname{rad} . \mathrm{T}^{-1} \cdot \mathrm{s}^{-1}$, where the anisotropy constant and saturation magnetization were chosen to match that of Ref. [62] and correspond to a small single-domain magnetic cube. We varied the reduced field $h$ in $[0.01,0.4]$ and computed an attempt frequency $f_{0}$ for each value of $h$. We obtained the graph in Fig. 3.6b, which shows good agreement with the analytical formula. This calculation was repeated with the perpendicular field applied along $z$, in order to validate the treatment of the poles, and yielded the same result.

\section{Skyrmion collapse}

We used the same parameters as given above for testing the skyrmion profile. The energy barrier for the isotropic collapse of the skyrmion was compared to one obtained with the implementations from Refs. [138] and [132]. The same barrier of $\Delta E=2.83$ $J_{\text {ex }}$ was found in all cases with good accuracy. The process of skyrmion collapse will be studied in more details in Chapter 4 .

\subsection{Summary}

In this chapter, we presented the extension of Kramers' method to a multidimensional phase space in the intermediate-to-high damping regime, as originally derived by Langer. Langer's theory provides an analytical expression of the attempt frequency for the rate of decay of a metastable state within the framework of reaction rate theory. The two main contributions to the attempt frequency are an entropic contribution, $\Omega_{0}$, which characterizes the change of phase space volume available to thermal fluctuations upon reaching the transition state, and a dynamical contribution, $\lambda_{+}$, which corresponds to the rate of growth of the (fastest growing) instability at the barrier top. 
We showed how the theory applies to magnetic spin systems and we derived an expression for the transition matrix of the LLG equation, from which $\lambda_{+}$is extracted as its largest positive eigenvalue.

Next, the principles behind the GNEB method were exposed. The GNEB method is a numerical scheme to relax paths of minimum energy between two energy minima on an energy surface. It is a version of the NEB method adapted to magnetic spin systems, in the sense that the curved manifold is taken into account by the use of geodesics to calculate distances, and by the projection of the tangent to the path and of the GNEB force onto the tangent space to the manifold. A minimum energy path constitutes a path of highest statistical weight, and therefore corresponds to a specific transition mechanism. Using a climbing image scheme, it is possible to precisely identify the highest first-order saddle point along the path. Once the saddle point is known, the internal energy barrier is known, and Langer's theory can be a applied in order to calculate a rate prefactor.

Finally, we gave some details on the numerical implementation of GNEB and Langer's theory into an atomistic code. The rate calculation was successfully tested against the analytical expression of the rate of reversal of a single macrospin under a perpendicular magnetic field within the IHD regime. The methods presented in this chapter will be used in the rest of this thesis to compute annihilation rates of metastable magnetic skyrmions. 


\section{Chapter 4}

\section{Thermal stability of chiral magnetic skyrmions from Langer's theory}

\subsection{Introduction}

In what follows, we apply Langer's theory adapted to magnetic spin systems to compute annihilation rates of chiral metastable magnetic skyrmions within the atomistic framework. The computation is carried out in two main steps. Firstly, the GNEB method is used to compute a minimum energy path through the energy landscape, and the saddle point is identified with high accuracy. Secondly, a rate prefactor is calculated according to Eq. (3.31). The results presented in this chapter are based on original publications [1] and [3].

The chapter is organized as follows. In Sec. 4.2 , we present the system under study and give the material parameters. In Sec. 4.3, we show paths to annihilation and corresponding energy profiles of isolated skyrmions via collapse and escape processes. After that, we compute transition rates associated with each mechanism in Sec. 4.4, and we consider the role of local eigenmodes and the entropic contribution to the prefactor. Finally, the results are discussed in Sec. 4.5 .

\subsection{Simulated system}

We simulate a two-dimensional system representing a thin magnetic film. The atomistic Hamiltonian is as defined in Sec. 2.1. The total simulated domain contains $50 \times 50$ spins, or $80 \times 80$ spins in the configuration involving two skyrmions, and we keep open (non-periodic) boundary conditions. The exchange interactions are limited to first nearest-neighbours. As we explained in Sec. 2.1.1, Néel-type skyrmions are stabilized by interfacial DMI, and Bloch-type skyrmions are stabilized by bulk-type DMI. 
As for antiskyrmions, they are favoured by modifying the interfacial DMI such that $\mathbf{D}_{i j}=-D \mathbf{r}_{i j} \times \mathbf{e}_{z}$ when $\mathbf{r}_{i j}=\mathbf{e}_{y}$ [139]. We use an isotropic exchange constant of $J_{\mathrm{ex}}=$ $1.6 \times 10^{-20} \mathrm{~J}(\sim 100 \mathrm{meV})$, which corresponds to an exchange stiffness of $A=16 \mathrm{pJ} / \mathrm{m}$ with lattice constant $a=1 \mathrm{~nm}$, and the saturation magnetization is set to $\mathscr{M}_{s}=1.1 \times 10^{6}$ A.m ${ }^{-1}$. These values correspond to typical $\mathrm{Pt} / \mathrm{Co}(0.6 \mathrm{~nm}) / \mathrm{AlO}_{\mathrm{x}}$ samples, in which interfacial DMI arises from the symmetry breaking at the interface [96]. The reduced parameters are then adjusted in order to stabilize small isolated metastable skyrmions at zero-field, with a diameter of approximately ten lattice sites, and are the following: $(b, d, k)=(0,0.36,0.4)[102]$. In what follows, we keep the external magnetic field to zero unless stated otherwise. The damping term in the LLG equation (Eq. (3.46)) is set to $\alpha=0.5$, which corresponds to commonly found values for ultrathin magnetic films with DMI [96], while pertaining to the IHD regime of Langer's theory. The gyromagnetic ratio is that of the free electron, $\gamma_{e}=1.76 \times 10^{11} \mathrm{rad} . \mathrm{s}^{-1} \cdot \mathrm{T}^{-1}$. The GNEB scheme is used on $Q=10$ or 15 images of the system for single-skyrmion mechanisms, and $Q=6$ images for the two-skyrmion mechanism.

\subsection{Paths to annihilation}

\subsubsection{Collapse mechanisms}

\section{Isolated skyrmion}

We first study the case of the collapse of a single isolated skyrmion. At zero-field, the core of the skyrmion coincides with a lattice site (image (im.) 1 in Fig. 4.1a). In that context, we report two separate mechanisms for the collapse of the Néel skyrmion. We show the spin profiles at selected images in Figs. $4.1 \mathrm{a}$ and $4.1 \mathrm{~b}$, and the corresponding energy profiles in Figs. $4.2 \mathrm{a}$ and $4.2 \mathrm{~b}$.

In the first case, which we refer to as mechanism $n^{0} 1$, the skyrmion progressively shrinks in size without breaking the cubic symmetry, while its core remains centred on a lattice site (Fig. 4.1a). This is accompanied by a slow increase in energy (Fig. 4.2a). At the saddle point (im. 8 in Fig. 4.1a, and inset in the middle in Fig. 4.2a), which we refer to as $S_{1}$, the core spin still points along $-z$, while its first neighbours are aligned in-plane. Once the core begins to reverse, the cubic symmetry is broken: the remaining spins flip, the energy drops dramatically and the system reaches the ferromagnetic ground state. The corresponding energy barrier is $\Delta E_{\mathrm{col}, 1}=2.83 \mathrm{Jex}_{\mathrm{ex}}$. This corresponds to about $10 k_{B} T_{300}$ with the present value of $J_{\text {ex }}$, where the notation $T_{300}$ is used to indicate $T=300 \mathrm{~K}$.

Alternatively, if mechanism $\mathrm{n}^{\circ} 2$ is realized, the skyrmion core may shift to an interstitial position in the centre of a square unit cell and, from there, the skyrmion 

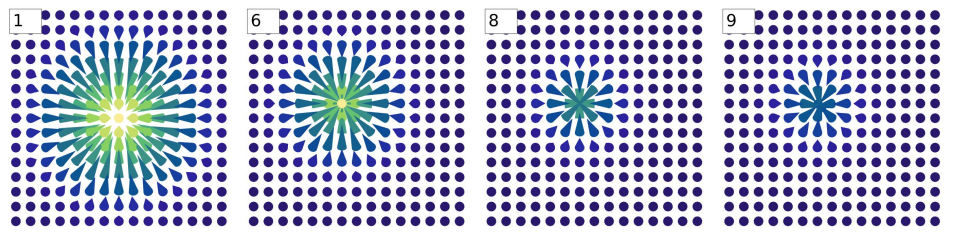

(a)

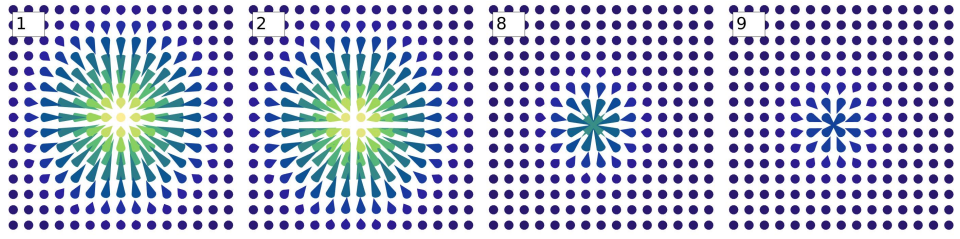

(b)
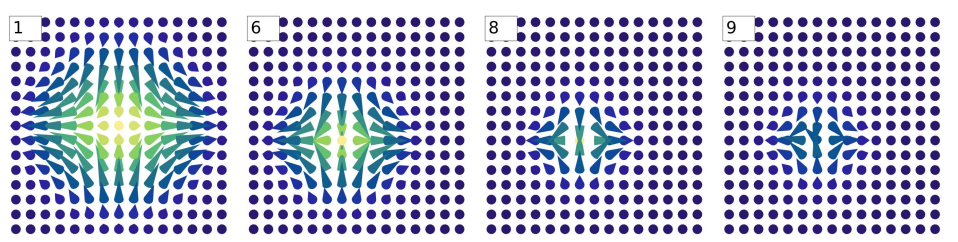

(c)

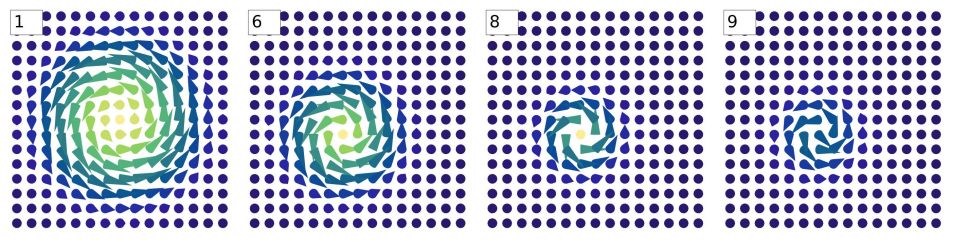

(d)

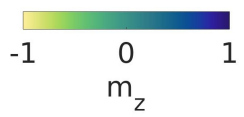

Figure 4.1: Spin maps of the isotropic collapse mechanisms. The index in the top lefthand corner corresponds to the image index of the GNEB method. We show $(a, b)$ for a Néel skyrmion, (a) collapse mechanism $\mathrm{n}^{0} 1$, and (b) collapse mechanism $\mathrm{n}^{0} 2$, followed by $(\mathrm{c}, \mathrm{d})$ mechanism $\mathrm{n}^{o} 1$ for (c) an antiskyrmion and, (d) a Bloch skyrmion.

uniformly shrinks in size (Fig. 4.1b). The saddle point configuration, which we call $S_{2}$, corresponds to the four central spins pointing towards the centre of the unit cell (im. 8 in Fig. $4.1 \mathrm{~b}$, and inset in Fig $4.2 \mathrm{~b}$ ). The energy profile is similar to that of the first mechanism. This particular path to collapse was first reported in Ref. [59]. In the present case, however, the translational modes at the SP become unstable, resulting in a third-order saddle point. For that reason, while the GNEB scheme is able to relax the path down to a low tolerance, the CI-GNEB scheme tends to shift it back to the first mechanism. We still manage to relax the path down to a tolerance on the GNEB force of $F_{\text {tol }}=9 \times 10^{-7}$. The influence of the set tolerance on the energy barrier and the attempt frequency is briefly discussed in Appendix A. The corresponding energy barrier is similar to that of the first mechanism: $\Delta E_{\mathrm{col}, 2}=2.90 \mathrm{Jex}_{\mathrm{ex}}$. Since the translational modes at the saddle point are not Goldstone modes [1,63], $S_{1}$ and $S_{2}$ should indeed be treated 


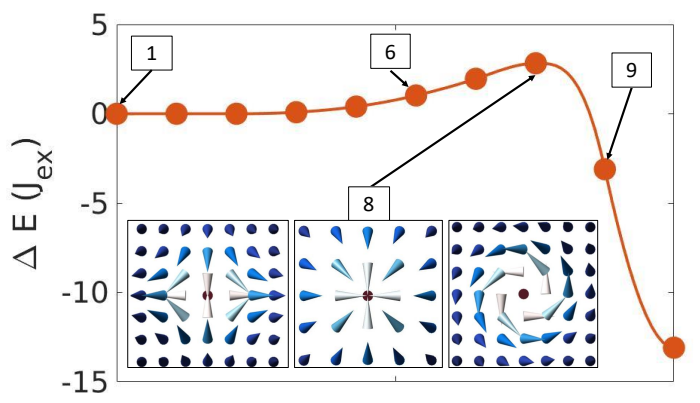

(a)

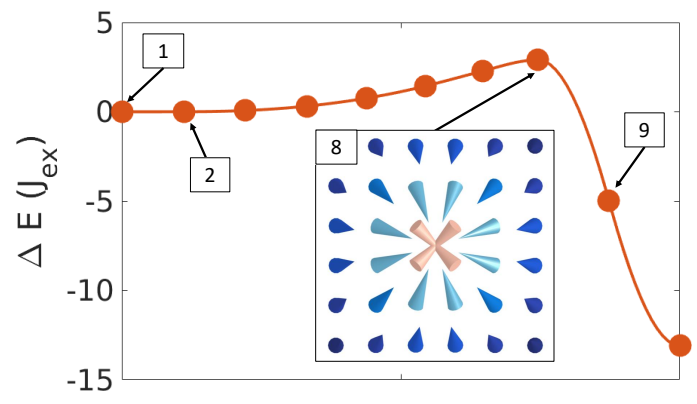

(b)

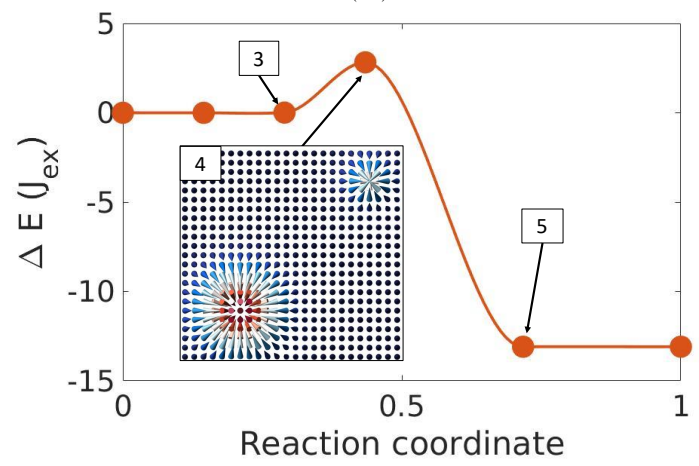

(d)

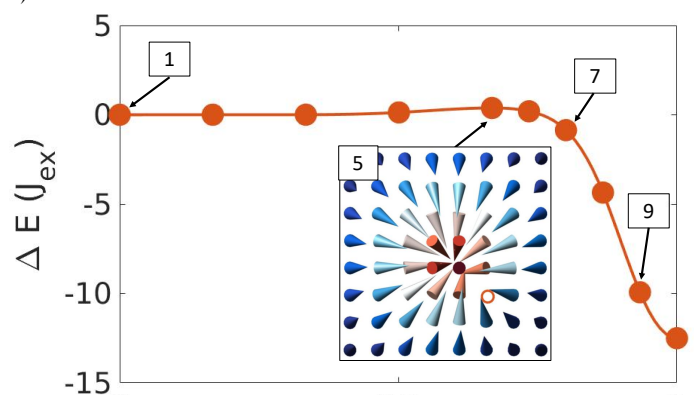

(c)

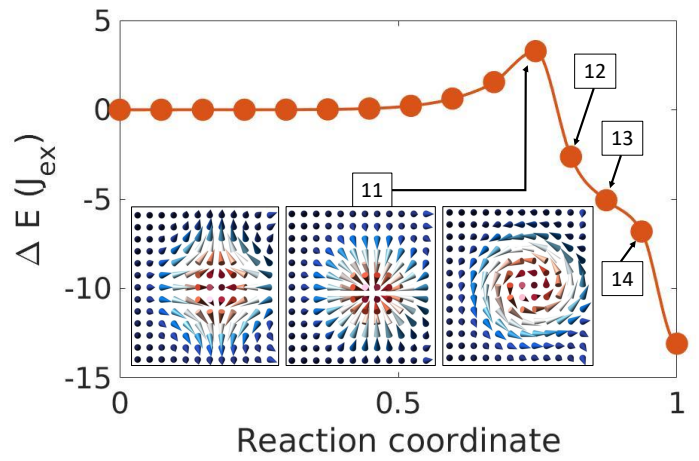

(e)

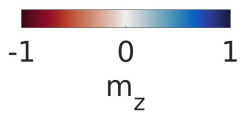

Figure 4.2: Interpolated energy profiles along the normalized reaction coordinate. Each dot corresponds to an image of the system on the energy surface. The insets show a closeup of the spin configuration at the saddle point. We show $(a, b)$ collapse mechanisms (a) $n^{o} 1$ and (b) $n^{0} 2$ of a single skyrmion, (c) collapse in the presence of a single non-magnetic defect, (d) collapse in the presence of another skyrmion and, (e) escape through a flat boundary. In (a) and (e), the inset respectively shows the saddle point configurations of the antiskyrmion, the Néel skyrmion, and the Bloch skyrmion.

as separate states, and they are therefore associated with different activation rates. $S_{2}$ is found above $S_{1}$ on the energy surface, by advancing along the eigenbasis coordinate associated with a translation mode.

Lastly, the collapse of an antiskyrmion and of a Bloch skyrmion, following a similar path to that of mechanism $\mathrm{n}^{\circ} 1$, are shown in Fig. $4.1 \mathrm{c}$ and $4.1 \mathrm{~d}$, respectively. They both exhibit a very similar behaviour to that of the Néel skyrmion, with a breaking of the 


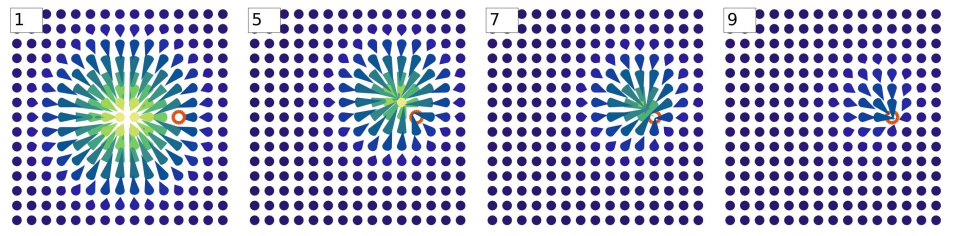

(a)

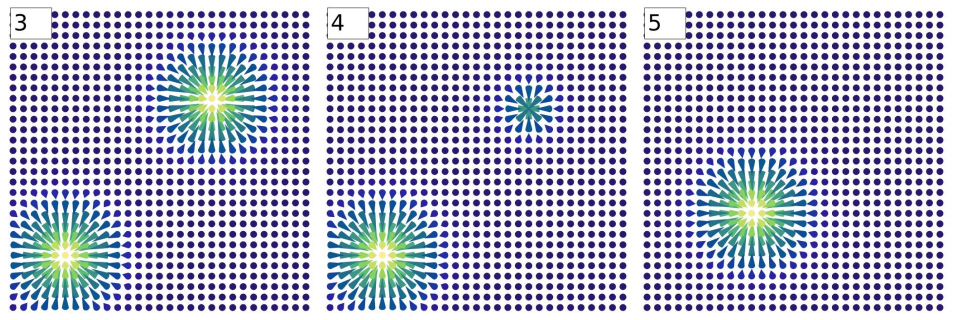

(b)

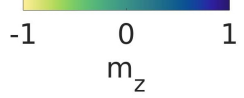

Figure 4.3: Spin maps of the collapse mechanisms of a Néel skyrmion in the presence of (a) a non-magnetic defect, where the position of the defect is indicated by a red circle, and (f) a second skyrmion. The conventions are the same as in Fig. 4.1.

symmetry past the saddle point corresponding to the flipping of the spin at the core. The energy profile along the path and the activation energy are the same for all three types of skyrmions. We did not calculate the collapse of the Bloch skyrmion and of the antiskyrmion via mechanism $n^{\circ} 2$, but we believe it would also be similar to that of the Néel skyrmion.

\section{Effect of a non-magnetic defect}

The effect of a single non-magnetic defect on the collapse was studied. This is important, because real systems are not perfect, and it has been experimentally observed that atomic defects are preferred sites for nucleation, pinning, and annihilation of skyrmions 33, 140]. In $\mathrm{Pd} / \mathrm{Fe} / \operatorname{Ir}(111)$, the interaction of skyrmions with atomic-scale defects was studied via SP-STM [140]. Native inlayer defects were found in the Pd layer and were assumed to be single Fe atoms due to intermixing. The defects were reported to be slightly off-centre compared to the pinned skyrmion. This was shown to be the case in bigger skyrmions either due to reduced spin stiffness or reduced DMI at the defect position, or due to reduced anisotropy for any skyrmion size. Here, we chose to follow Ref. [141] by simulating a non-magnetic defect. This is done by setting the anisotropy to zero at a given site, as well as all exchange interactions with the neighbouring sites. Im. 1 in Fig. 4.3 a shows that the skyrmion relaxes such that the defect sits where the spins lie in-plane, which reproduces experimental observations [140]. The simulated skyrmion is small, so the off-centre pinning is likely due to a reduction 
in the anisotropy energy. We show spin maps at selected images in Fig. 4.3a, and the corresponding energy profile in Fig. 4.2c. As the core moves towards the defect - which takes place along the diagonal of the square lattice - the skyrmion shrinks in size. This costs little energy compared to the isotropic collapse mechanism, as seen on the energy profile of Fig. 4.2c. The saddle point is im. 5 on the path and corresponds to a larger skyrmion than in the defect-free configuration, but the skyrmion is deformed and rendered asymmetric by the defect. Consistent with results presented in Ref. [141], the presence of the defect significantly lowers both annihilation and nucleation barriers, and we obtain $\Delta E_{\mathrm{def}}=0.38 \mathrm{~J}_{\mathrm{ex}}$, which is one order of magnitude lower than $\Delta E_{\mathrm{col}, 1,2}$.

\section{Effect of a second skyrmion}

We consider what happens when two skyrmions get close to each other. We show spin maps at selected images in Fig. 4.3b, and the corresponding energy profile in Fig. $4.2 \mathrm{~d}$. When the skyrmion cores are initially aligned along the lattice directions $x$ or $y$, we observe that they rotate in order to approach each other along the lattice diagonal. Presumably, this is more energetically favourable due to the choice of first-neighbour interactions on the simple square lattice. Consequently, we simply initialize the skyrmions diagonally from each other. Note that this may be less true in real systems with corrections in the exchange to include interactions beyond the first neighbours, and in systems where the skyrmions are big compared to the lattice parameter and decouple from the lattice. Additionally, interface skyrmions tend to be observed in systems with a hexagonal lattice, for instance $\mathrm{Pd} / \mathrm{Fe}$ and $\mathrm{Rh} / \mathrm{Co}$ on $\operatorname{Ir}(111)$ [33, 118], in which these considerations do not apply. We set the transition path for a merging of the two skyrmions into one, as observed experimentally in the case of the decay of a skyrmion lattice into the helical state [46]. However, the search for a first-order SP consistently results in a switch in mechanism, and the collapse of one of the skyrmions is relaxed instead of the merging (Fig. 4.3b). This behaviour hints at the fact that the merging mechanism involves a higher-order SP, which may therefore involve a higher barrier and be less favourable than the collapse. It was confirmed by a mode following method [142] that, with our present set of parameters, the merging mechanism is indeed associated with a higher order SP. However, for other parameters, merging may in fact traverse a first-order SP [142].

The mechanism is as follows. The skyrmions initially approach one another, which at first costs almost no energy (im. [1-3] in Fig. 4.2d), until a critical distance is reached at which the collapse of the upper skyrmion is initiated. The saddle point is the same as that of collapse mechanism $\mathrm{n}^{0} 1$ for the upper skyrmion, while the other one remains stable (im. 4 in Fig. 4.3b, and inset in Fig. 4.2d). At finite temperature, isolated skyrmions perform a diffusive Brownian motion on the lattice under the effect of thermal 
fluctuations [143]. Although the interaction potential between chiral skyrmions is repulsive at all distances [25], some fluctuations may bring them close to each other. In that case, it is interesting to study how the collapse rate is affected. The activation energy for collapse remains the same: $\Delta E_{2 \mathrm{sk}}=2.82 \mathrm{~J}$. We shall see how the attempt frequency is modified in Sec. 4.4 .

\subsubsection{Escape through a boundary}

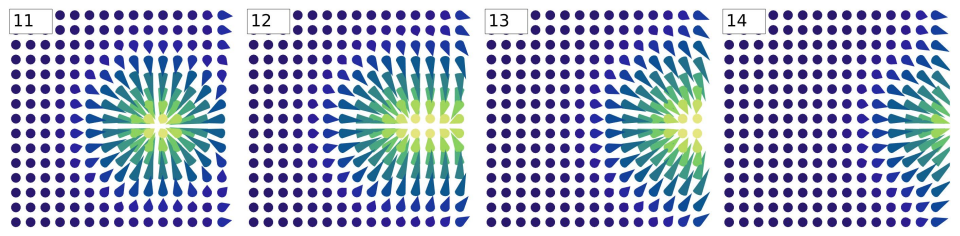

(a)

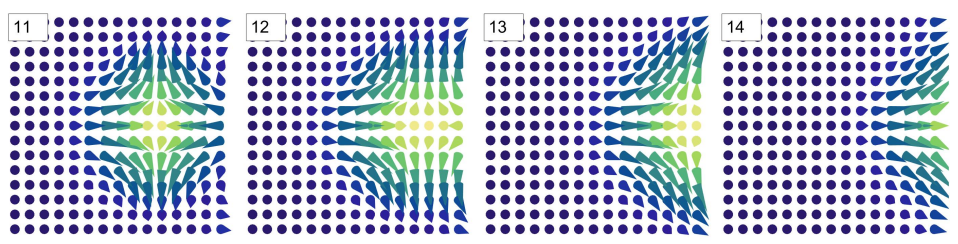

(b)
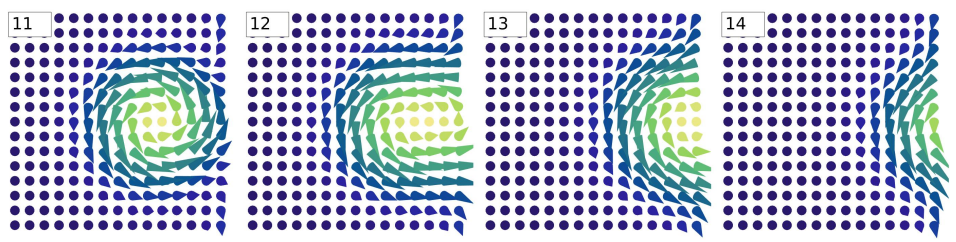

(c)
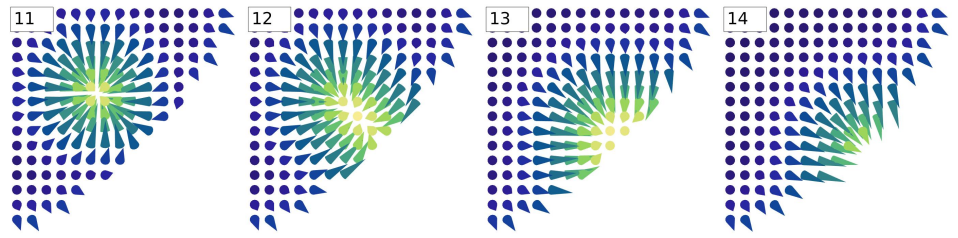

(d)

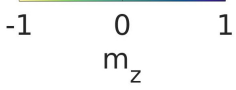

Figure 4.4: Spin maps of the escape mechanisms. The conventions are the same as in Fig. 4.1. On all the subfigures, the saddle point is im. 11, which is the state where the skyrmion sits tangent to the boundary. We show (a) a Néel skyrmion, (b) an antiskyrmion, (c) a Bloch skyrmion, and (d) a Néel skyrmion escaping through a curved boundary. 


\section{Flat boundary}

Escape through a boundary is another possible path in finite-sized systems. We show spin maps at selected images in Figs. $4.4 \mathrm{a}, 4.4 \mathrm{~b}$ and $4.4 \mathrm{c}$ respectively for a Néel skyrmion, an antiskyrmion and a Bloch skyrmion, and the corresponding energy profile in shown in Fig. 4.2e. Because of DMI, the spins are tilted at the boundary with the same chirality as that of the skyrmion. For the same reason that chiral skyrmions repel each other, the boundary is also repulsive to the skyrmion [36, 107], such that the total energy increases as the skyrmion leaves the centre of the lattice and moves towards an edge (ims. [1-11] in Fig. 4.2e). For the Néel skyrmion, the antiskyrmion, and the Bloch skyrmion, the saddle point corresponds to a position where the skyrmion sits adjacent to the boundary (im. 11 in Fig. $4.4 \mathrm{a}, 4.4 \mathrm{~b}$ and $4.4 \mathrm{c}$, respectively, and insets in Fig. 4.2e), as was also shown in Ref. [141]. Past the saddle point, the skyrmion deforms and elongates as it comes in contact with the edge and begins to disappear. This is accompanied by a large decrease in the energy. Im. 13 in Fig. $4.4 \mathrm{a}, 4.4 \mathrm{~b}, 4.4 \mathrm{c}$ and $4.2 \mathrm{e}$ corresponds to a half-skyrmion - or meron - sitting on the edge. In the vicinity of this point, the decrease in energy appears to slow down, before speeding up again as the rest of the remaining skyrmion disappears. The activation energy obtained for this mechanism is the highest one of the three processes studied here, although they are all of a similar magnitude of 2-3 $J_{\mathrm{ex}}: \Delta E_{\mathrm{esc}}=3.28 J_{\mathrm{ex}}$, once again for all three types of skyrmions. While it should be noted that in the present configuration, this mechanism possesses four equivalent realisations (one at each side of the square), we shall not include a factor of four in the attempt frequency, as the escape rate through through one edge is potentially more relevant that the total escape rate out of a square sample.

\section{Curved boundary}

Fig. 4.4d shows the escape of a Néel skyrmion through a curved boundary. For that purpose, a disc-shaped system is obtained from the square system by converting outer spins into non-magnetic sites. The nature of the discrete lattice means that the boundary exhibits a staircase effect, and so the spin lattice as a whole only exhibits a cubic-type of symmetry, as opposed to a radial symmetry. Note that the boundary is considered curved if the curvature is perceived at the scale of the skyrmion, which is the case here. The escape process is similar to that of the flat boundary configuration, but the activation energy is found to be slightly higher: $\Delta E_{\text {curv }}=3.60 J_{\text {ex }}$. The energy profile is similar to that of Fig. $4.2 \mathrm{e}$, and so we do not show it. 


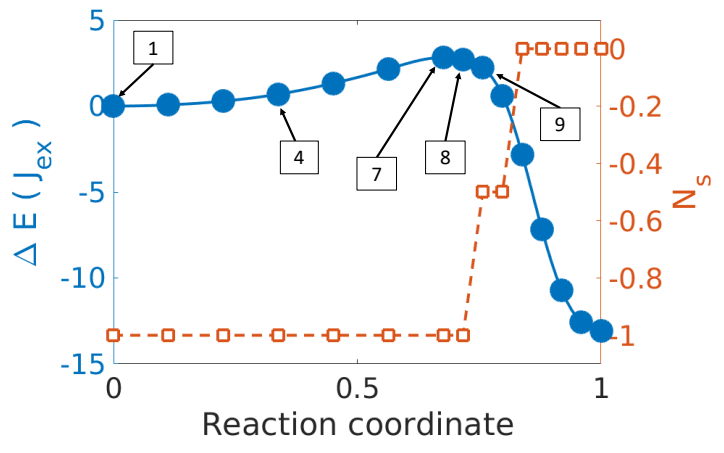

(a)

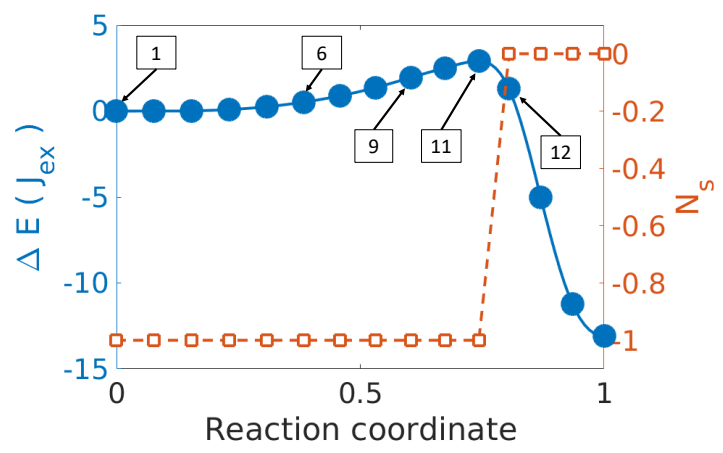

(b)

Figure 4.5: Interpolated energy profile and evolution of the topological charge $N_{s}$ along the reaction coordinate for collapse mechanisms (a) $\mathrm{n}^{o} 1$ and (b) $\mathrm{n}^{o} 2 . N_{s}$ is calculated according to Eq. (2.38).
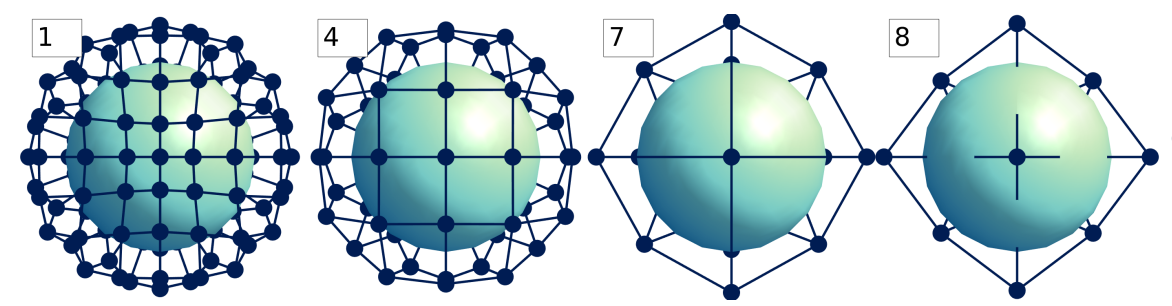

(a)
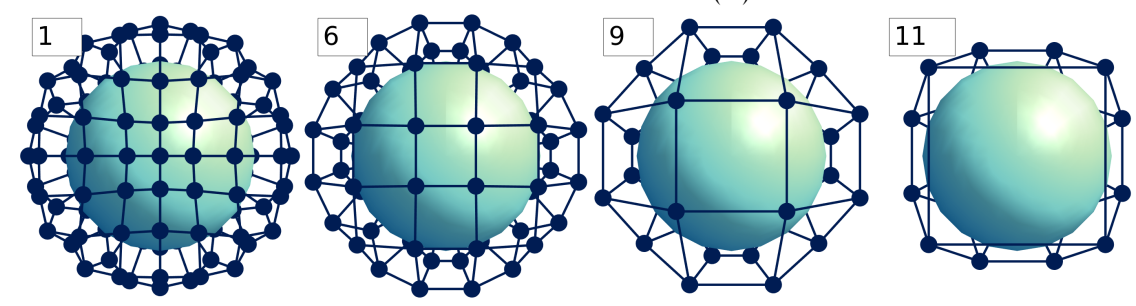

(b)
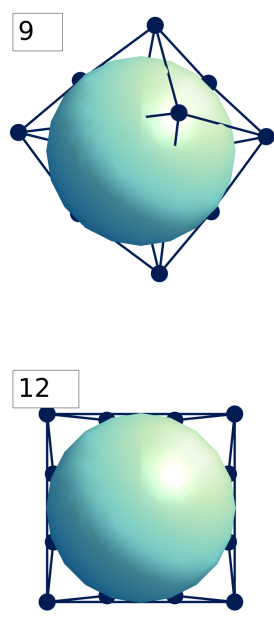

Figure 4.6: "Ball in a net" representation of the spin configuration at selected images along the path for collapse mechanisms (a) $n^{o} 1$ and $(b) n^{o} 2$. The image index is given in the top left-hand corner. The topological charge on the discrete spin lattice is defined as the number of times the magnetization net wraps around the unit sphere, so this representation shows the evolution of the topological charge. 


\subsubsection{Paths to collapse and evolution of the topological charge}

We compare the evolution of the topological charge $N_{s}$ along the path for collapse mechanisms $\mathrm{n}^{0} 1$ and 2. To do so, we compute a value of $N_{s}$ at each image using the definition from Eqs. (2.38) and (2.39). We plot the result in Fig. 4.5 as well as the energy profiles. We now use $Q=15$ images to improve the definition of the path in the area where the topological charge drops. In Fig. 4.6, we use the "ball in a net" type of representation from Sec. 2.3.2 to show the spin configuration at different images along the MEP. As previously described in Sec. 2.3.2, vertices correspond to the tip of the magnetic vectors with their origin in the centre of the sphere, and the edges represent the exchange coupling between first neighbours. The ferromagnetic background $($ spins along $+z)$ is found at the north pole of the sphere, while the core of a skyrmion typically points along the south pole (along $-z)$. The view is set just below the south pole, looking axially towards the $+z$ direction, such that the core of the skyrmion is at the centre of the view. Since we defined the topological charge on the discrete lattice as the number of times the magnetization "net" wraps around the unit sphere, this type of representation provides an intuitive way to visualise the evolution of the topological charge.

For mechanism $\mathrm{n}^{0} 1$, we find that the topological charge is conserved past the saddle point, which is im. 7 (Figs. $4.5 \mathrm{a}$ and $4.6 \mathrm{a}$ ), and until im. 8. At ims. 9 and 10, the core spin starts to flip, and we find a half-integer topological charge: $N_{s}=-0.5$. After that, the topological charge vanishes and the configuration becomes topologically trivial.

On the other hand, during the second mechanism, the topological charge drops from $N_{s}=-1$ to 0 at im. 12, which is the image directly following the one at the saddle point (im. 11 in Figs. $4.5 \mathrm{~b}$ and 4.6b). However, a half-integer value may exist as a punctual state for this mechanism (punctual in the sense that this state would have zero-length along the reaction coordinate) and would correspond to the case where the four spins in the centre point exactly in-plane.

Therefore, the two collapse mechanisms provide an example of two different ways through which an integer topological charge may be annihilated without using the edges.

\subsection{Transition rates from Langer's theory}

\subsubsection{Overview}

Once the saddle point is accurately identified along a path, the corresponding rate prefactor can be calculated according to Langer's theory (Eq. (3.31)). All terms entering 
(a) Collapse

\begin{tabular}{cccccccc}
\hline \hline Mechanism & $\Delta E\left(J_{\text {ex }}\right)$ & $\Omega_{0, \text { int }}$ & $\begin{array}{c}\Omega_{0, \text { tot }} \\
\left(\times 10^{-5}\right)\end{array}$ & $\begin{array}{c}\frac{\Omega_{0, \text { int }}}{\Omega_{0, \text { tot }}} \\
\left(\begin{array}{cccc}\lambda_{+} \\
(\mathrm{GHz})\end{array}\right.\end{array}$ & $\begin{array}{c}f_{0} \\
(\mathrm{MHz})\end{array}$ & $\begin{array}{c}k_{300} \\
(\mathrm{kHz})\end{array}$ \\
\hline single sk. 1 & 2.83 & 0.0015 & 3.51 & 43 & 1200.47 & 6.70 & 0.12 \\
single sk. 2 & 2.90 & 0.00048 & 1.05 & 46 & 2124.23 & 3.54 & 0.05 \\
defect & 0.38 & 0.0214 & 116 & 18 & 145.41 & 26.90 & 6190.40 \\
two sk. & 2.82 & 0.0009 & 2.32 & 42 & 1200.23 & 4.43 & 0.08 \\
\hline \hline
\end{tabular}

(b) Escape

\begin{tabular}{cccccccc}
\hline \hline Mechanism & $\begin{array}{c}\Delta E \\
\left(J_{\text {ex }}\right)\end{array}$ & $\Omega_{0, \text { int }}$ & $\begin{array}{c}\Omega_{0, \text { tot }} \\
\left(\times 10^{-3}\right)\end{array}$ & $\begin{array}{c}\Omega_{0, \text { int }} \\
\Omega_{0, \text { tot }}\end{array}$ & $\begin{array}{c}\lambda_{+} \\
(\mathrm{GHz})\end{array}$ & $\begin{array}{c}f_{0} \\
(\mathrm{MHz})\end{array}$ & $\begin{array}{c}k_{300} \\
(\mathrm{kHz})\end{array}$ \\
\hline flat bound. & 3.28 & 0.0349 & 12.4 & 2.8 & 522.94 & 1036.2 & 3.25 \\
curved bound. & 3.60 & 0.0198 & 4.48 & 4.4 & 501.45 & 357.2 & 0.32 \\
\hline \hline
\end{tabular}

Table 4.1: Terms of the rate prefactor and total annihilation rate at $T=300 \mathrm{~K}$ for (a) collapse processes and (b) escape processes. From left to right, we give the internal energy barrier, $\Delta E$, in units of the isotropic exchange constant, $J_{\mathrm{ex}}$, the entropic contribution to the prefactor, $\Omega_{0}=\Omega_{0, \text { tot }}$, the dynamical contribution to the prefactor, $\lambda_{+}$, the Arrhenius prefactor, $f_{0}$, and the total rate of annihilation at room temperature, $k_{300}=k(\mathrm{~T}=300 \mathrm{~K}) . \Omega_{0 \text {,int }}$ gives the contribution of internal modes to the prefactor and $\Omega_{0 \text {,tot }}$ gives the total contribution of all modes. Their ratio shows that the main contribution comes from the internal modes. $f_{0}$ and $k_{300}$ are calculated using $\Omega_{0}=\Omega_{0 \text {,tot }}$. The entropic contribution, $\Omega_{0}$, is found to be much smaller than one, which is a stabilizing contribution. This is the reason why the obtained attempt frequencies are typically found in the $\mathrm{MHz}$ regime, which is uncharacteristically low for magnetic structures.

in the calculation of activation rates are summarized in Table 4.1 for each mechanism. In Fig. 4.7, we show the evolution of the average lifetime of the skyrmion, $\tau=k^{-1}$, associated with each annihilation mechanism as a function of temperature. We find that the collapse on a non-magnetic defect yields by far the shortest lifetime. At low temperature, collapse mechanisms are found more probable than escape, but this trend reverses at higher and room temperature, where the longest lifetimes are associated with collapse. In the absence of defects, the average lifetimes at room temperature $(300 \mathrm{~K})$ are found between $0.3 \mathrm{~ms}$, which is associated with the escape through a flat boundary, and $21 \mathrm{~ms}$, which corresponds to collapse mechanisms $\mathrm{n}^{\circ} 2$. Collapse mechanism $\mathrm{n}^{o} 1$ and escape through a flat boundary were studied for the Néel and Bloch skyrmions, as well as the antiskyrmion, and yield the same results. In the cases of escape through a curved boundary, collapse mechanism $\mathrm{n}^{\circ} 2$, and collapse on a defect, we studied only the Néel skyrmion, but it seems reasonable to assume the following results also hold for other types of skyrmion solutions. A possible issue of non-negligeable numerical rounding errors affecting the accuracy of the ratio of eigenvalues was previously men- 


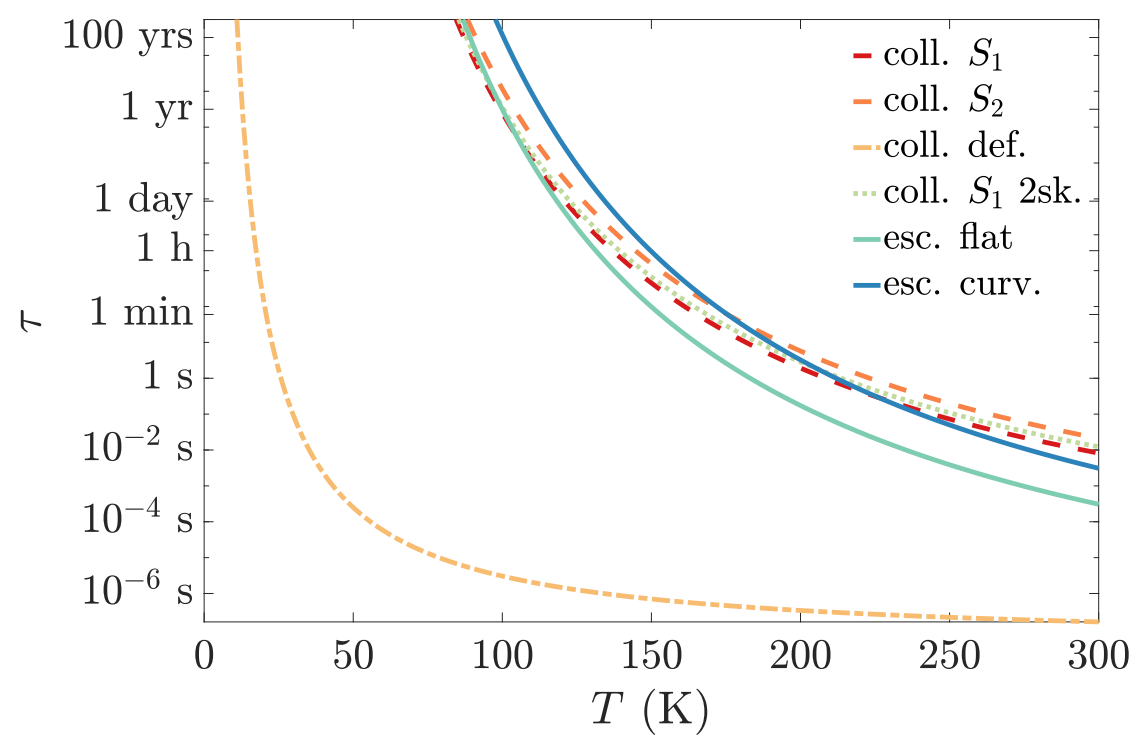

Figure 4.7: Average lifetime of a skyrmion as a function of temperature with respect to all considered mechanisms calculated via Langer's theory as given in Eq. (3.31). The energy barriers and the detail of all the different contributions to the prefactor are given in Table 4.1. Here, the collapse on a non-magnetic defect yields by far the shortest lifetime. At low temperature, collapse mechanisms are found more probable than escape, but this trend reverses at room temperature, where the longest lifetimes are associated with skyrmion collapse. In the absence of defects, the average lifetimes at room temperature $(300 \mathrm{~K})$ are found between $0.3 \mathrm{~ms}$ (escape through a flat boundary) and $21 \mathrm{~ms}$ (collapse mechanisms $\mathrm{n}^{\circ} 2$ ).

tioned in [62]. In Table A.1 of Appendix A, we gather results of simulations performed for different lattice sizes. We find that, as long as the skyrmion is not constrained by the boundary, the size of the lattice does not significantly affect the computed attempt frequencies, and therefore there seems to be no significant error originating from numerical noise in our results.

\subsubsection{The thermal role of internal eigenmodes}

\section{Mode classification}

For each mechanism considered here, the curvatures of the energy surface at $A$ and $S$ are ordered by increasing amplitude with corresponding index $i$, and plotted in Fig. 4.8 for all $i$ and in Figs. 4.9 ( a $-\mathrm{c}, \mathrm{j}-1)$ for the first 15 or 25 values of $i$. Figs. 4.9(d$\mathrm{f}, \mathrm{m}-\mathrm{o})$ show the ratio of individual curvatures, $\lambda_{i}^{A} /\left|\lambda_{i}^{S}\right|$, plotted in semilog scale. The value of

$$
\Omega_{0, i}=\sqrt{\prod_{j=1}^{i} \frac{\lambda_{j}^{A}}{\left|\lambda_{j}^{S}\right|}}
$$



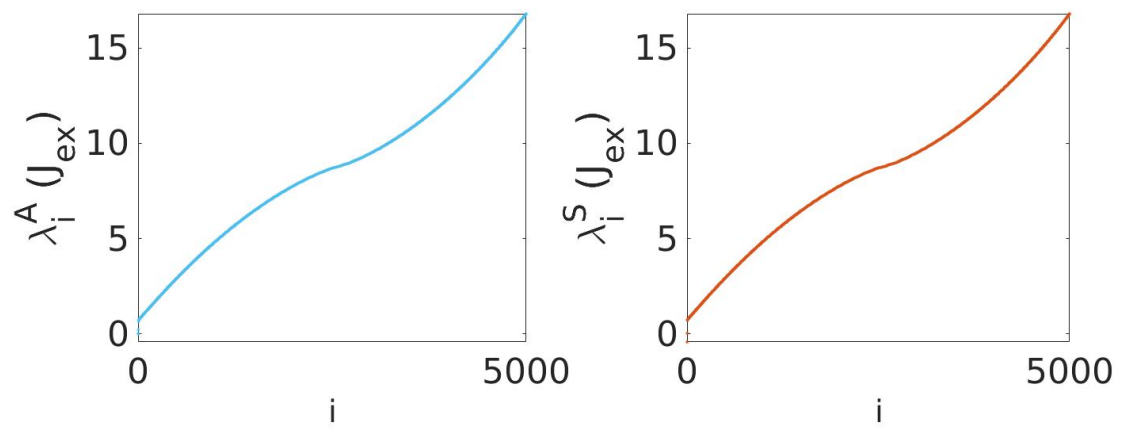

Figure 4.8: All energy curvatures at $A$ (in blue) and $S$ (in red) ordered by increasing amplitudes for the case of the collapse of a single skyrmion via mechanism $\mathrm{n}^{0} 1$. The other mechanisms exhibit the same profiles which we interpret as the dispersion of spin-wave excitations, with the exception of the first few eigenvalues. These are found below the main curve and are shown in Figs. $4.9(\mathrm{a}-\mathrm{c}, \mathrm{j}-\mathrm{l})$

is plotted in a similar fashion in Figs. $4.9(\mathrm{~g}-\mathrm{i}, \mathrm{p}-\mathrm{r})$, where all the curvatures are shown in the inset. We note that for all mechanisms, the ratio of curvatures only shows significant variations for small $i$ and weak variations for $i \gg 1$. Consequently, the value of $\Omega_{0, i}$ shows a strong $i$-dependence at small $i$ and a weak dependence for larger $i$. More specifically, for all collapse mechanisms, $\Omega_{0, i}$ shows a strong $i$-dependence for small $i$, a medium dependence for intermediate $i$ and a weak dependence for large $i$. In the case of escape mechanisms, the $i$-dependence decreases at low and intermediate indices, and goes up again in the domain of highest curvatures.

From Eq. (3.13), the relative amplitudes of small fluctuations about the extrema for each mode $i$ are given by the components of the corresponding eigenvector, $\chi_{i}$. This allows us to plot the spatial profiles for the $\theta$-eigenmodes at $A$ and $S$ in Figs. 4.10 and 4.11. The $\phi$-profiles exhibit similar behaviour and do not provide any further information for the following analysis. In recent years, the eigenmodes of skyrmions have been extensively investigated [144-154]. Localized modes were reported to exist below the magnon continuum, with excitations such as translational motion, uniform breathing, elliptic and triangular distortions, etc. Classifications based on the azimuthal number $m$ were proposed, which is linked to the number of nodes $2|m|$ encountered when going around the skyrmion centre in the direction of increasing azimuth angle $\varphi$. In this work, we use the $i$-index of the ordered eigenfrequencies (Fig. 4.9) to classify the modes. While some relation to spin waves calculated in previous work could be made, our classification scheme is useful because it allows comparison of eigenvalues and eigenfunctions of the fluctuations around both the metastable state and the saddle point. 

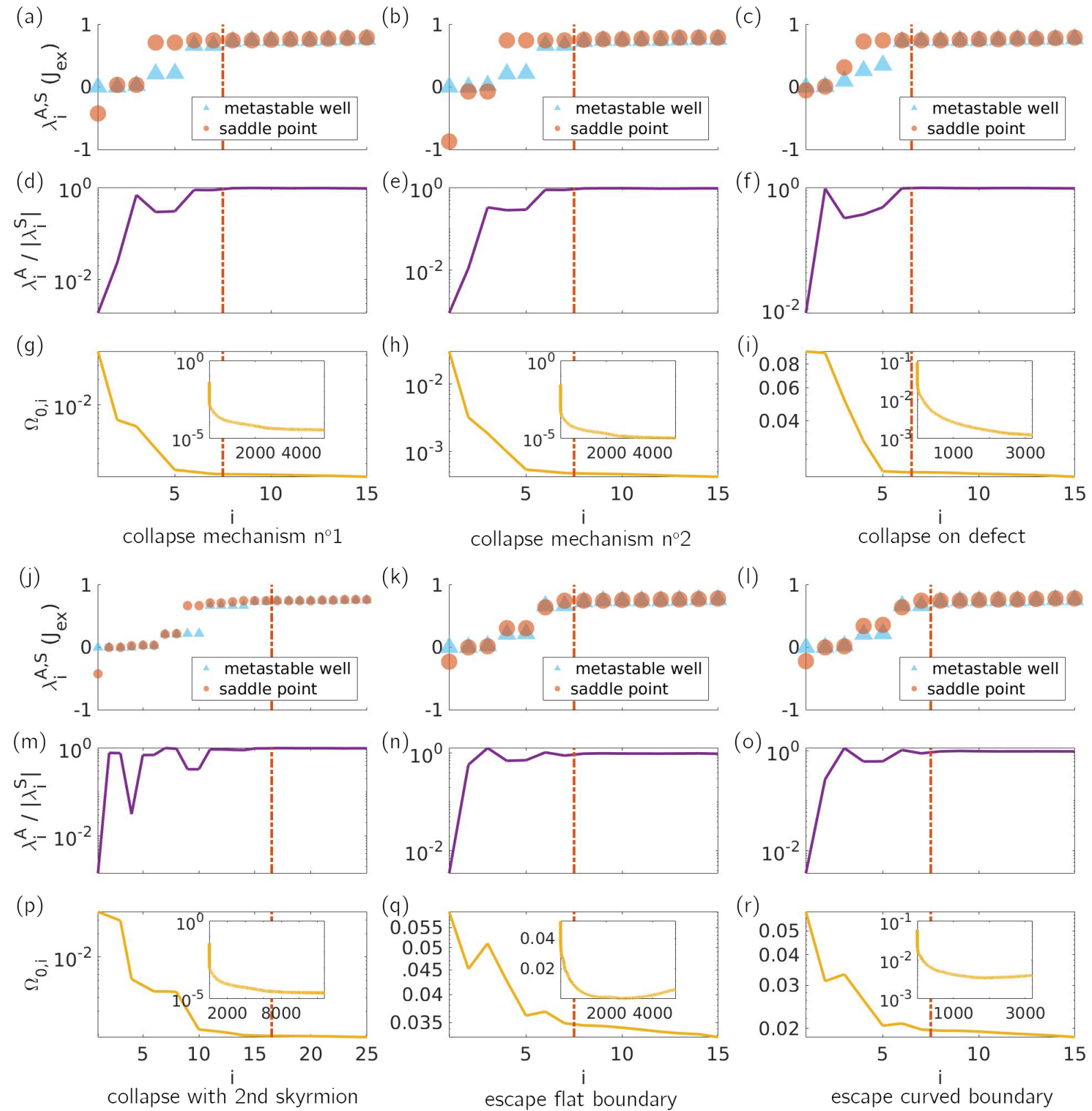

Figure 4.9: Detail of the computation of the entropic contribution to the prefactor, $\Omega_{0}$, for different annihilation mechanisms. The mechanism in question in indicated at the bottom of each set of three graphs. $(a-c, j-1)$ We show the first 15 or 25 eigenvalues of the Hessian matrix of the energy evaluated at $A$ (in blue) and $S$ (in red). The eigenvalues are in unit of the isotropic exchange constant $J_{\mathrm{ex}}$ and ordered by increasing amplitudes, where the index $i$ is attributed accordingly. A negative eigenvalue corresponds to an unstable mode of fluctuations. The red dotted line marks the separation between localized and collective eigenmodes. $(\mathrm{d}-\mathrm{f}, \mathrm{m}-\mathrm{o})$ Ratio of individual eigenvalues in semilog scales. The ratio of eigenvalues associated with local modes (left-hand side of the red line) is typically different from one, while for collective modes (righthand side of the red line) it is close to one. $\left(\mathrm{g}-\mathrm{i}, \mathrm{p}-\mathrm{r}\right.$ ) Cumulative $\Omega_{0, i}$ as defined in Eq. (4.1) in semilog scale, where the inset shows its value for all $i=1 \ldots 2 N$. The value of $\Omega_{0}$ used in the prefactor is $\Omega_{0,2 N}$. The local modes contribute the most to $\Omega_{0}$, and $\Omega_{0} \ll 1$, which is a stabilizing contribution to the attempt frequency. 


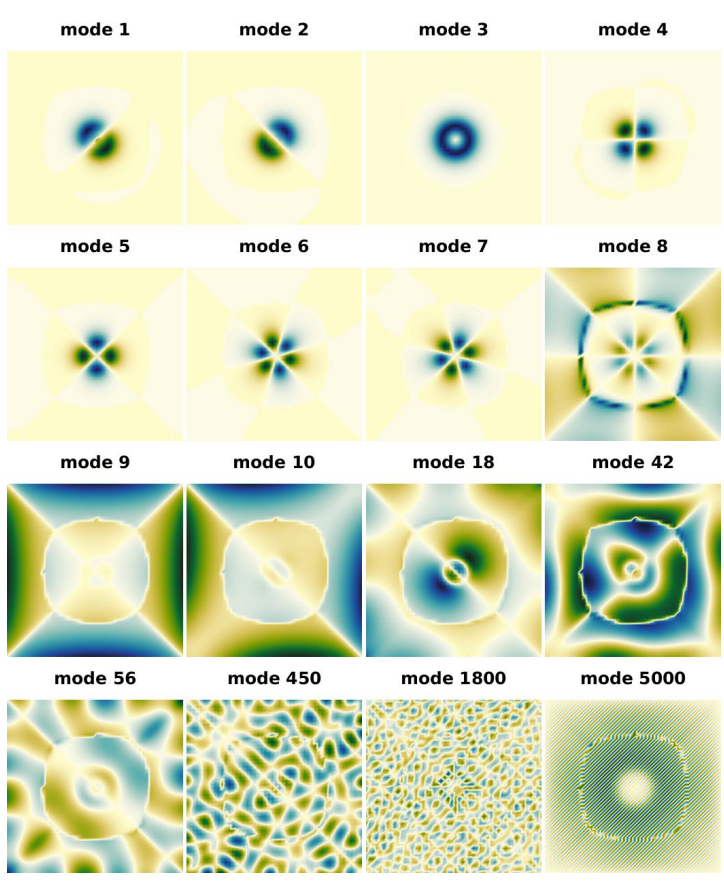

(a)

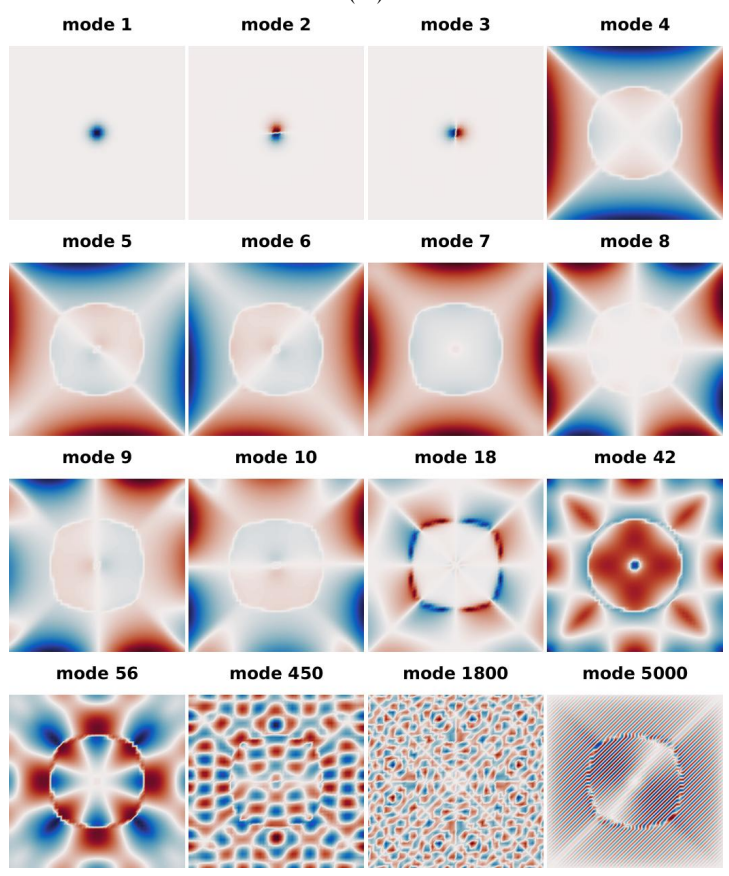

(c)

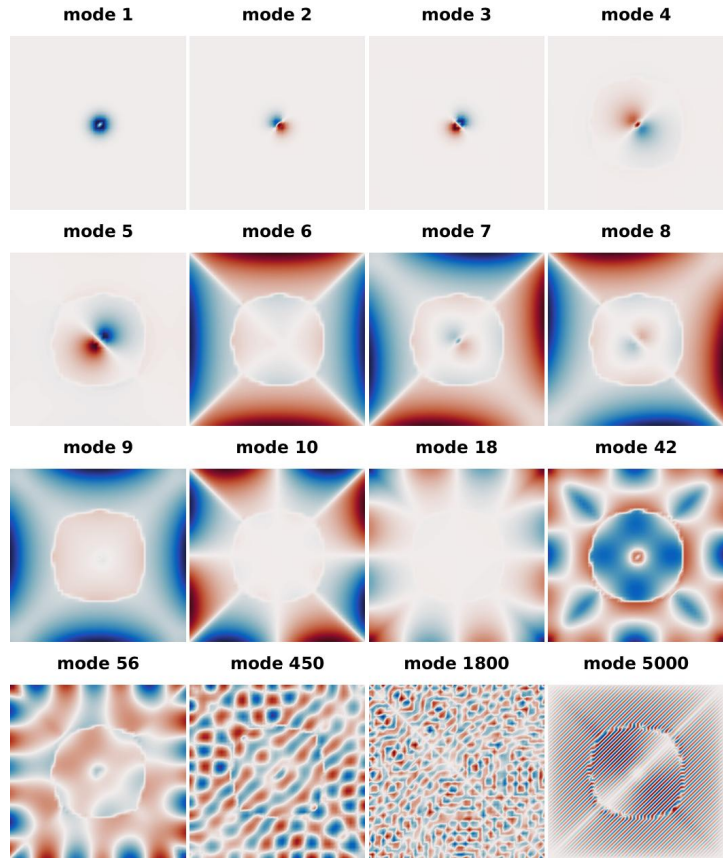

(b)

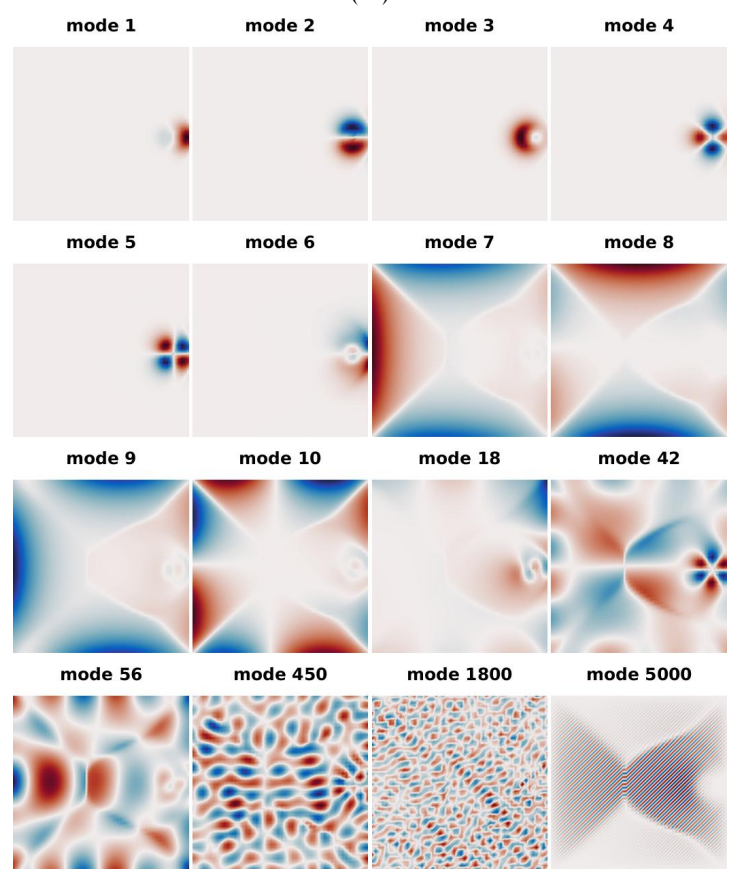

(d)

Figure 4.10: Eigenmodes associated to the $\theta$ variable for a single skyrmion in a simulated system of $50 \times 50$ spins. The blue and green colour scheme is associated with metastable states and the blue and red one with saddle points. Negative amplitudes are plotted in blue and positive ones in green/red. The range of the colour map is adjusted on each plot so that zero-amplitude fluctuations coincide with white. Modes are designated via the $i$-index of the corresponding ordered eigenfrequencies of Fig. 4.9. We designate by $i_{\text {col }}$ (short for "collective") the index of the first non-local mode. We show (a) the metastable single-skyrmion state with $i_{c o l}=8$, (b) the saddle point of collapse mechanism $\mathrm{n}^{0} 1$ with $i_{c o l}=6$, (c) the saddle point of collapse mechanism $\mathrm{n}^{o} 2$ with $i_{c o l}=4$, and $(\mathrm{d})$ the saddle point of the escape mechanism with $i_{c o l}=7$. 


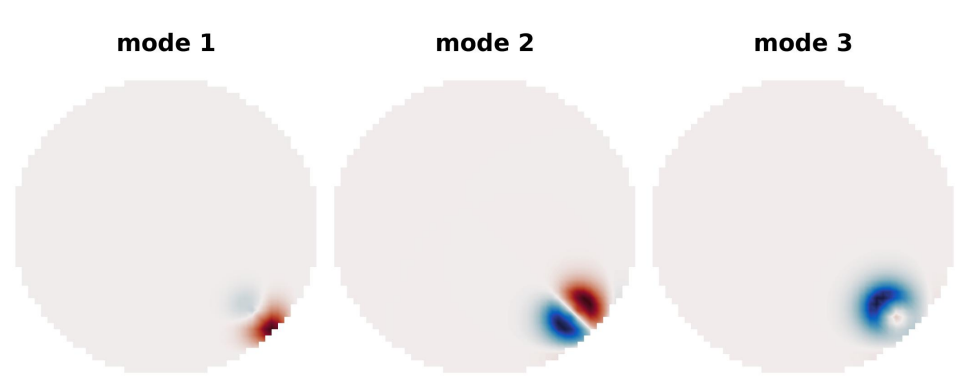

(a)

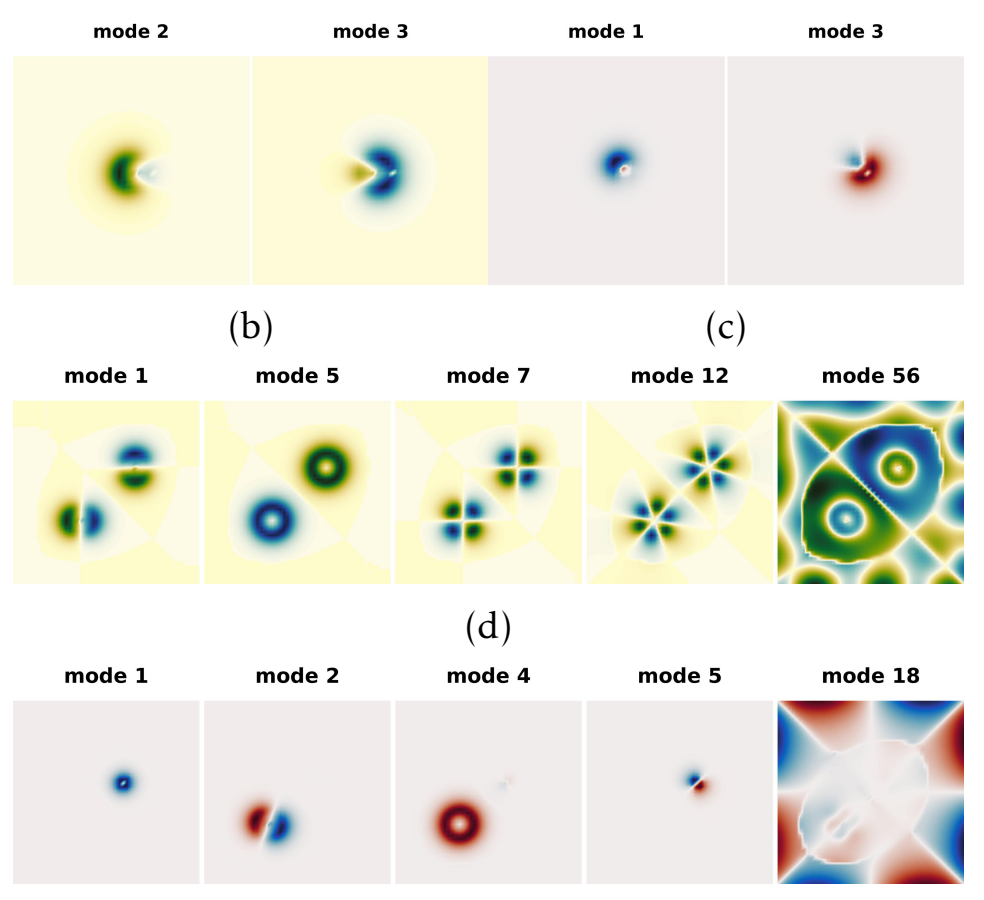

(e)

Figure 4.11: Eigenmodes associated to the $\theta$ variable. The colour code is the same as in Fig. 4.10. We show (a) some modified internal modes for the escape through a curved boundary at $S,(\mathrm{~b}, \mathrm{c})$ some modified internal modes for the collapse on a defect at $A$ and $S$, and $(\mathrm{d}, \mathrm{e})$ some internal and collective modes of two coupled skyrmions on a $50 \times 50$ lattice at $A$ and $S$.

\section{Local and collective modes}

In Figs. 4.10 and 4.11 , and in agreement with previous studies, the first clear observation is that for all cases, the lowest energy eigenmodes are localized, internal skyrmion modes. The rest of the modes are collective modes extended to the entire lattice and can be thought of as amplitudes corresponding to spin-wave (SW) excitations.

Firstly, from Fig. 4.10a, the first two modes of the metastable skyrmion state are translational modes $(m= \pm 1)$. These are not zero-modes because the lattice breaks the translational invariance in this case, however the corresponding eigenvalues are close to zero (see Fig. 4.9a, where the eigenvalues at $A$ are shown as blue triangles). Mode 3 is the uniform breathing mode $(m=0)$ and is a low energy mode slightly above modes 
1 and 2 in Fig. $4.9 \mathrm{a}$. The next two modes directly above them correspond to elliptic distortions of the skyrmion shape $(m= \pm 2)$ and the final two local modes are triangular distortions $(m= \pm 3)$. Therefore, there are seven local states of the metastable well. The subsequent modes are part of the magnon continuum.

At the saddle points $S_{1}$ and $S_{2}$ of the collapse mechanisms (Figs. $4.10 \mathrm{~b}$ and $4.10 \mathrm{c}$ ), the breathing mode becomes unstable. At $S_{2}$, the translational modes are also unstable. In total, five local states exist at $S_{1}$, and three at $S_{2}$.

At the escape saddle point (Fig. 4.10d), the unstable skyrmion exhibits similar modes to that of its metastable counterpart, but they appear distorted by the presence of the edge. Six local modes are found. The unstable mode (mode 1) is a mode of translation towards the boundary, and is the mode that enables the escape of the skyrmion. The edge lifts the degeneracy of the translational modes: mode 2 is a mode of translation parallel to the boundary, and the associated eigenvalue is close to zero (see Fig. $4.9 \mathrm{k}$, where the eigenvalues at $S$ are shown as red circles) as this displacement costs little energy. Whether the boundary is flat or curved (Fig. 4.11a), the same number of internal modes exist at $A$ and $S$, and they appear similar. There are, of course, fewer collective modes associated with the circular sample, since there are fewer magnetic sites and therefore fewer degrees of freedom in that case.

Next, Figs. $4.11 \mathrm{~b}$ and $4.11 \mathrm{c}$ display examples of how the presence of a defect modifies some of the internal modes. In particular, the unstable breathing mode at the saddle point is significantly affected by the defect (Fig. 4.11c). The defect also lowers the total number of internal modes, as six internal modes survive at $A$, and four at $S$.

Lastly, in the system of two metastable coupled skyrmions, which we show in Fig. $4.11 \mathrm{~d}$, the internal modes are the same as that of a single skyrmion and both skyrmions are excited simultaneously, which results in a total of 16 internal modes. This is no longer the case at the transition state (Fig. 4.11e) where all the amplitude is localized to only one of the two skyrmions in each of the internal modes. Note that Figs. 4.11d and $4.11 \mathrm{e}$ show modes on the $50 \times 50$ lattice, whereas calculations were carried out on the $80 \times 80$ lattice to prevent the skyrmions from being constrainted by the edge.

\section{Contribution of the modes to the prefactor}

The internal modes that we have described above correspond, in fact, to the modes found below the magnon continuum in Figs. $4.9(\mathrm{a}-\mathrm{c}, \mathrm{j}-\mathrm{l})$. The separation between localized and collective modes is shown by a dashed line in Fig. 4.9, and also coincides with the transition between strong and low $i$-dependence of $\Omega_{0, i}$ (Figs. 4.9 (g $-\mathrm{i}, \mathrm{p}$ $-r)$ ). The contribution of the internal modes to the prefactor is given in Table 4.1 by $\Omega_{0 \text {,int }}$, while the complete contribution of all the modes corresponds to $\Omega_{0 \text {,tot }}$. For the collapse mechanisms, the values differ by a factor of $\approx 40$ between them, or 18 when 
a defect is present. In the case of the escape processes, the ratio is a factor of three (flat boundary), or four (curved boundary). In other words, the relative contribution of internal modes varies depending on the annihilation process, and is found to be higher for collapse processes compared to escape processes. It is reduced if a defect is present, and increases if the boundary is curved. Hence, the relative contribution of internal and collective modes is strongly linked to the nature of the annihilation and the geometry of the transition state. Most importantly, internal modes play the most significant role in the thermally activated annihilation of a skyrmion. Each of the collective spin-wave modes brings a weak contribution, but because there are many more collective modes than there are internal modes, their contribution to the attempt frequency should not be neglected.

\section{Broken symmetries}

Finally, we note that the eigenmodes at the saddle point tend to display broken symmetries compared to the metastable states. In the single metastable skyrmion case (Fig. 4.10a), the internal modes in particular possess symmetries of types two-fold, four-fold, six-fold, and radial. At the saddle point of the collapse (Fig. 4.10b), the four-fold and six-fold symmetries are absent. As for the saddle configuration at the boundary (Fig. $4.10 \mathrm{~d}$ and 4.11a), symmetries are broken by the edge. Lastly, in the case of the two skyrmions, the symmetry breaking at the SP in Fig. $4.11 \mathrm{e}$ is striking, since each internal mode involves only one skyrmion, in contrast to the metastable eigenmodes in Fig. $4.11 \mathrm{~d}$, which involve both skyrmions.

\subsubsection{Entropic contribution to skyrmion stability}

\section{Variation of the rate between different paths to annihilation}

From the results shown in Table 4.1 and Fig. 4.7, a striking observation is that the most probable mechanisms do not necessarily correspond to the lowest energy barriers. For instance, for the small skyrmions considered here, escape mechanisms involve a higher barrier than collapse mechanisms, yet, at room temperature, escape is more probable. Therefore, activation energies alone do not allow the lifetime of skyrmions to be properly estimated. Characteristic times at the transition state were found to lie in the $\mathrm{GHz}-\mathrm{THz}$ regime, which remains in the range of typically assumed values for estimates of the prefactor in magnetic spin systems [64-67]. However, a large difference is observed here due to the contribution of the ratio of curvatures, $\Omega_{0}$, which significantly lowers the attempt frequency. As was explained in Sec. 3.2.2, $\Omega_{0}$ characterizes the entropic contribution to the prefactor. By inverting Eq. (3.39), we can plot the change in configurational entropy, $\Delta S=S_{S}-S_{A}$, as a function of temperature. This is shown 


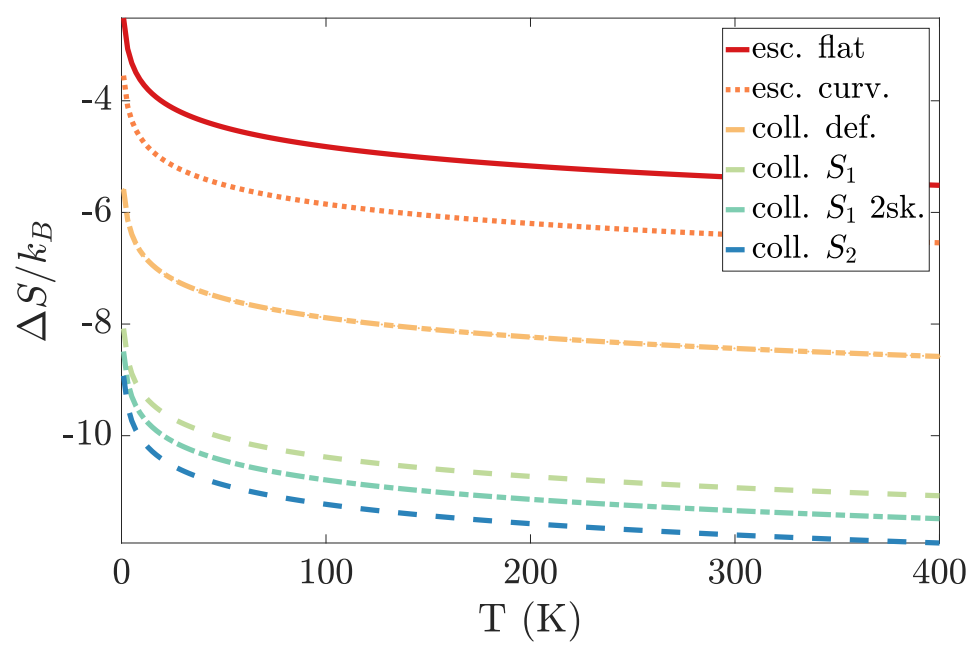

Figure 4.12: Calculated change in configurational entropy induced when the system goes to the saddle point $\Delta S / k_{B}=\left(S_{S}-S_{A}\right) / k_{B}$ as defined in Eq. (3.38) over a broad range of temperatures for all mechanisms. The highest entropic barrier corresponds to the most negative $\Delta S$, i.e. the collapse involving the saddle point $S_{2}$.

in Fig. 4.12 for all previously considered mechanisms. In all cases, we find $\Delta S<0$ : the configurational entropy of the metastable state is higher than that of the saddle point. This result implies that the number of micro-realisations of the metastable skyrmion state is higher than that of the transition state. A potential source of stability of individual skyrmions might therefore lie in lowered attempt frequencies due to entropic narrowing in the saddle point region, rather than in high internal energy barriers. The smaller reduction in entropy between $A$ and $S$ in the boundary annihilation may be explained by the fact that the transition state is a full skyrmion, which remains somewhat similar to the metastable skyrmion state. From Eq. (3.39), $\Omega_{0}(i)$ plotted in Figs. 4.9 $(\mathrm{g}-\mathrm{i}, \mathrm{p}-\mathrm{r})$ in semilogarithmic scale behaves as $\Delta S(i)$. Hence, the change in entropy is primarily associated with the internal modes of a skyrmion.

The results in Table 4.1 and Fig. 4.7 show that at room-temperature, escape processes tend to be more probable than collapse, with the exception of the collapse on a defect. This occurs because, even though the energy barriers for collapse are lower, the attempt frequencies are also lower. While, at low temperature, the rate is dominated by the internal energy barrier, at higher temperature entropic effects contained in the attempt frequency are more important. If the attempt frequencies for all mechanisms were of a similar order of magnitude of a few gigahertz, collapse processes would remain the most probable mechanisms at all temperatures, and the average lifetime associated with collapse in the absence of defects would span between about 50 to $70 \mu$ s. In reality, it lies between 8 and $21 \mathrm{~ms}$, which differs from the predicted result by three orders of magnitude.

The escape through a curved boundary was found to be less likely than through a 
flat boundary, due to both the internal energy barrier and the entropic barrier being increased at the curved boundary. We also stress that the skyrmions we have considered so far only span over a few lattice sites and are stabilized at zero-field. For different stabilization processes involving an external field and lower perpendicular anisotropy, escape processes may be found more favourable also in terms of the internal energy barrier [141]. Interestingly, the collapse in the presence of another skyrmion exhibits the same internal energy barrier as in the case of a single skyrmion, but a higher entropic barrier. The presence of a second skyrmion thus results in a lowered attempt frequency for collapse: $f_{0}^{\text {col }}=4.43 \mathrm{MHz}$ for the collapse via mechanism $\mathrm{n}^{\circ} 1$ when a second skyrmion is present, as opposed to $f_{0}^{\mathrm{col}}=6.70 \mathrm{MHz}$ for a single isolated skyrmion. This may be explained by the fact that the two skyrmion modes are coupled in the metastable state, and so 16 internal modes exist in total, which results in a larger configurational entropy in the metastable well, and consequently a lowered attempt frequency for collapse compared to the single skyrmion case. Finally, the presence of a non-magnetic defect significantly affects all terms of the transition rate. Firstly, as seen in Fig, 4.2c, it lowers the internal energy barrier by almost one order of magnitude, rendering it practically flat. It also decreases the entropic contribution to the prefactor by two orders of magnitude, that is, the entropic barrier is lowered (see also Fig. 4.12). Thirdly, the rate of growth of the unstable mode at the saddle point is decreased by one order of magnitude. This effect could, in theory, be stabilizing, but we find it is negligible against the significantly lower value of the total activation energy. It can also be noted that the internal energy barrier for the nucleation is lowered as well. This is consistent with experimental observations that skyrmions tend to nucleate and annihilate near defects [140].

\section{Variation of the collapse rate as a function of applied field}

We now consider the effect of an external magnetic field applied along the $+z$ direction, so that it points opposite to the core of the skyrmion and therefore has a destabilizing effect. We focus on the collapse processes of a single skyrmion. For reduced field values $b_{z} \in[0,0.05]$, we relax the corresponding collapse mechanisms $\mathrm{n}^{0} 1$ and 2 . With our present set of parameters, this corresponds to a field variation from 0 to about 730 $\mathrm{mT}$. The relaxed metastable skyrmion core may either be centred on a site (State $A$ in Fig. 4.13a), or at an interstitial position (State $A$ in Fig. 4.13b). This is determined by its equilibrium size and its commensurability with the underlying lattice. If, for a given field value, we attempt to relax the skyrmion at a different position on the lattice - i.e., centred on a site if it initially relaxed at an interstitial point, and vice versa - it will relax back to its initial position. Therefore, there seems to exist only one type of stable skyrmion state $A$ per field value, although if we apply periodic boundary conditions, 


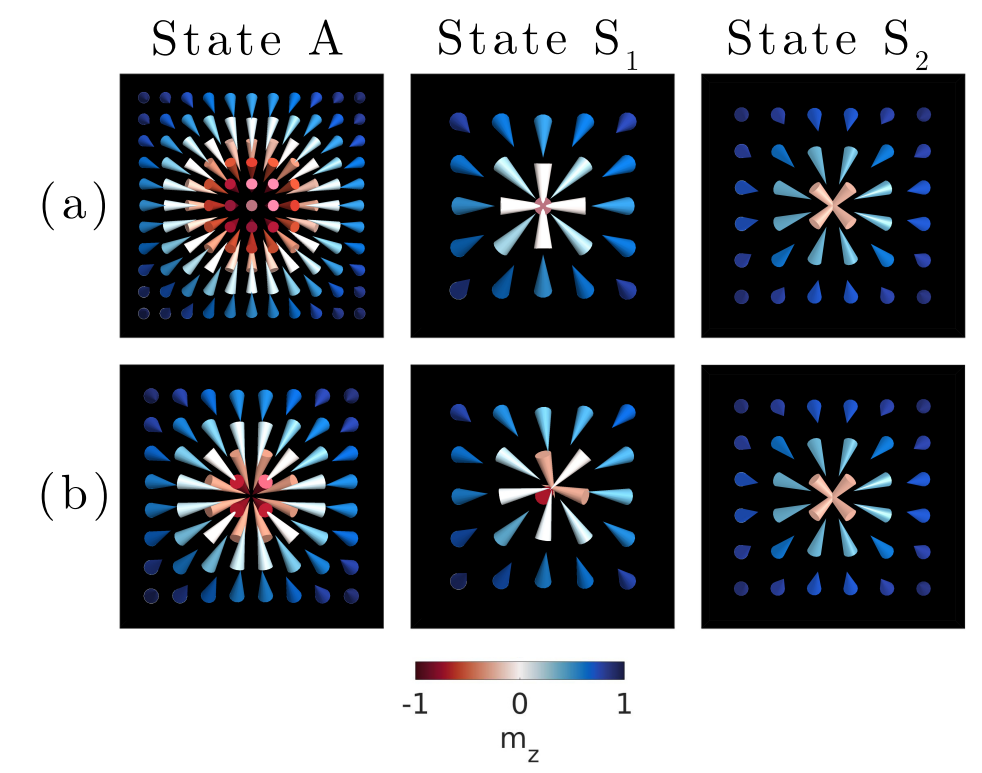

Figure 4.13: Spin maps (zoomed) of the metastable state $A$ and saddle points $S_{1}$ and $S_{2}$ of the skyrmion collapse for (a) $b_{z}=0$, and (b) $b_{z}=0.05$. $S_{1}$ corresponds to the skyrmion core centred on a lattice site, while $S_{2}$ corresponds to the core located at an interstitial point.

the skyrmion may of course be indistinguishably located at either of the $N$ possible sites. If the metastable skyrmion is pinned at an interstitial point, the realisation of mechanism $n^{o} 1$ requires the core to firstly shift onto a lattice site. For all field values, $S_{2}$ is found to be of order three. With this in mind, the rate associated with mechanism $\mathrm{n}^{\circ} 2$ will contain some degree of error (see Table A.3), as we are not able to relax the CIGNEB force below a certain tolerance. That tolerance varies depending on the applied field strength $\left(9 \times 10^{-7}<F_{\text {tol }}<6 \times 10^{-5}\right)$.

As we increase the field, the stable skyrmion size decreases - in agreement with theoretical and experimental studies [108, 155, 156] - and so does the internal energy barrier for both collapse mechanisms, which we show in Fig. 4.14a. The energy barriers associated with $S_{1}$ and $S_{2}$ are almost identical and decrease smoothly with the applied field. Note that the barrier at $S_{2}$ is slightly higher than at $S_{1}$.

In Fig. $4.14 \mathrm{~b}$, we plot the change in entropy, $\Delta S_{1,2}=S_{S_{1,2}}-S_{A}$, induced by respectively reaching $S_{1}$ and $S_{2}$, as defined in Eq. (3.38) for a first-order saddle point such as $S_{1}$, and more generally in Eq. (3.40) for a $k$ th-order saddle point. This latter case is relevant for $S_{2}$, for which $k=3 . \Delta S_{1,2}$ is found to increase as the field increases. Since $\Delta S_{1,2}<0$ for all field values, the entropic contribution remains stabilizing, as it lowers the attempt frequency, but this stabilizing contribution decreases at higher field values. We also find that $\Delta S_{2}<\Delta S_{1}<0$, so $S_{S_{2}}<S_{S_{1}}$ : from an entropic perspective, $S_{1}$ is more likely to be visited by the system.

In Fig. $4.14 \mathrm{c}$, we show the value of the dynamical contribution to the prefactor at 
saddle points $S_{1}$ and $S_{2}$, namely $\lambda_{+, 1}$ and $\lambda_{+, 2}$, as a function of the applied field. We find that they decrease weakly when the field increases. $\lambda_{+, 2}$ is slightly larger than $\lambda_{+, 1}$ and decreases more than $\lambda_{+, 1}$. For both of them, we consistently obtain a value in the range of a few terahertz, with little variation as a function of the applied field, i.e., $0.8<\lambda_{+, 1}<1.2 \mathrm{THz}$ and $1.15<\lambda_{+, 2}<2.12 \mathrm{THz}$. Following the discussion in Sec. 3.3.2, since $S_{2}$ is a third-order saddle point, $\lambda_{+, 2}$ is chosen as the rate of growth of the fastest growing instability [125].

If we assume that collapse mechanisms $\mathrm{n}^{o} 1$ and 2 are independent, the total rate of collapse of the skyrmion can be expressed as

$$
k_{\mathrm{tot}}^{\mathrm{col}}(T)=k_{1}(T)+k_{2}(T)=f_{01} e^{-\beta \Delta E_{1}}+f_{02} e^{-\beta \Delta E_{2}},
$$

where $f_{01}$ and $f_{02}$ are the attempt frequencies respectively associated with mechanisms $\mathrm{n}^{o} 1$ and 2 . We should note that since the difference in internal energy between $S_{1}$ and $S_{2}$ is quite small (around $0.08 J_{\text {ex }}$ at zero field), the mechansisms are probably not completely decoupled. In Fig. 4.14d, we plot the total collapse rate calculated with Eq. (4.2), as well as the individual rates $k_{1}$ and $k_{2}$. For each field value, we set the value of $T$ such that $\beta \Delta E_{1}=10$. We find that $k_{1}$ is much larger than $k_{2}$, so that $k_{1}+k_{2} \approx k_{1}$, despite the fact that the internal energy barriers are almost identical. In the higher field region $\left(0.04 \leq b_{z} \leq 0.05\right)$, the configuration at $S_{1}$ loses cubic symmetry (i.e., invariance under $\pi / 2$-rotations of the system about the $z$-axis) as seen in Fig. $4.13 \mathrm{~b}$, and possesses four equivalent realisations. They can be visualized by picturing rigid $\pi / 2$-rotations of the system. However, the non-symmetric state $S_{2}$ corresponds to a small deviation $\delta$ from the symmetric state. If the amplitude of the thermal fluctuations exceeds $\delta$, then the four states cannot be resolved at finite temperature and, on average, should correspond to a single state. For this reason, we do not include a factor of four in the rate. This choice will be further confirmed in Chapter 5.

Lastly, since the energy barriers involved in both mechanisms are very similar (Fig. 4.14a), we can recover an approximate total attempt frequency for the collapse as $f_{0, \text { tot }}^{\text {col }} \approx f_{01}+f_{02}$, where the energy barrier is approximated as $\Delta E_{1} \approx \Delta E_{2} \approx \Delta \bar{E}$, with

$$
\Delta \bar{E}=\frac{\Delta E_{1}+\Delta E_{2}}{2}
$$

The attempt frequencies $f_{01}, f_{02}$, and $f_{0 \text {,tot }}^{\text {col }}$ are plotted in Fig. $4.14 \mathrm{e}$ as a function of the reduced field. $f_{0 \text {,tot }}^{\text {col }}$ spans over three orders of magnitude, that is, $10.2 \mathrm{MHz} \leq f_{0 \text {,tot }}^{\text {col }} \leq$ 23.24 GHz. In particular, 6.7 $\mathrm{MHz} \leq f_{01} \leq 22.8 \mathrm{GHz}$, and $3.5 \mathrm{MHz} \leq f_{02} \leq 2.68 \mathrm{GHz}$. This shows a strong dependence of the attempt frequency on the applied magnetic field. The dynamical contribution is bound in the THz region and does not explain why the attempt frequency extends from the $\mathrm{MHz}$ to the $\mathrm{GHz}$ regime. This effect has also 
been discussed in Refs. [46, 63, 157] and once again finds its origin in the strong entropic contribution to the prefactor, as shown in Fig. $4.14 \mathrm{~b}$. Additionally, we typically obtain $f_{0,2} \ll f_{0,1}$, which in turns explains why the total rate $k_{\text {tot }}^{\text {col }}$ is dominated by $k_{1}$ even though $\Delta E_{1} \approx \Delta E_{2}$. Once again, since $\lambda_{+, 1}<\lambda_{+, 2}$, this is a direct consequence of the different entropic contributions, more specifically $\Delta S_{2}<\Delta S_{1}<0$. We note that, since the skyrmions are not decoupled from the lattice, we observe strong lattice effects that result in non-monotonic variations of the entropic contribution (Fig. 4.14b), which are in turn found in the variations of the attempt frequency (Fig. 4.14e) and the total collapse rate (Fig. 4.14d). 


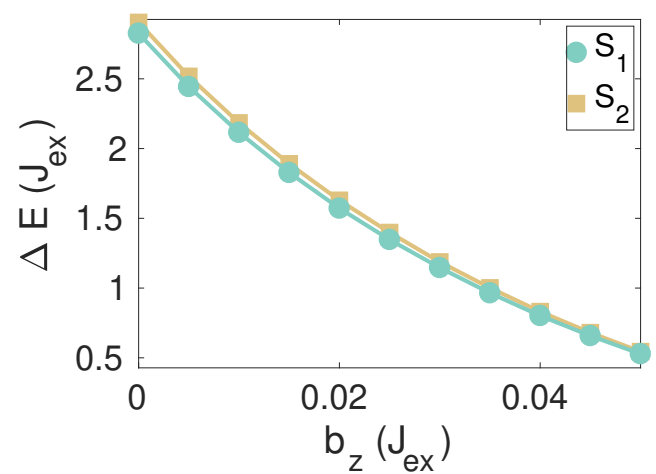

(a)

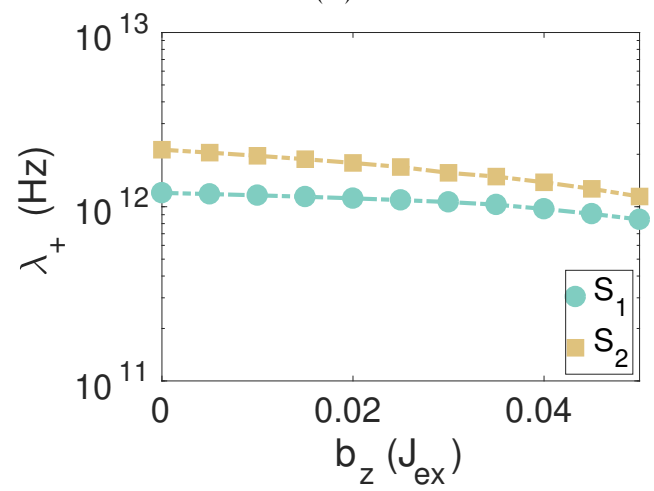

(c)

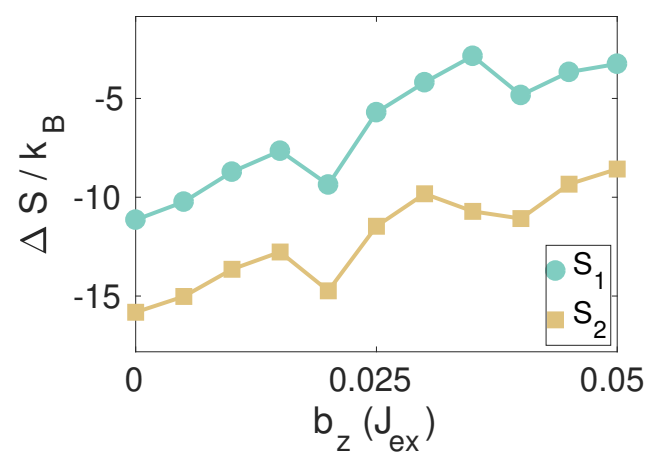

(b)

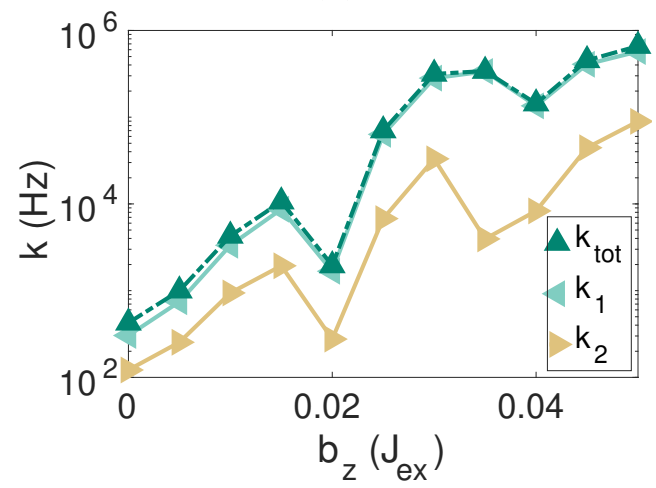

(d)

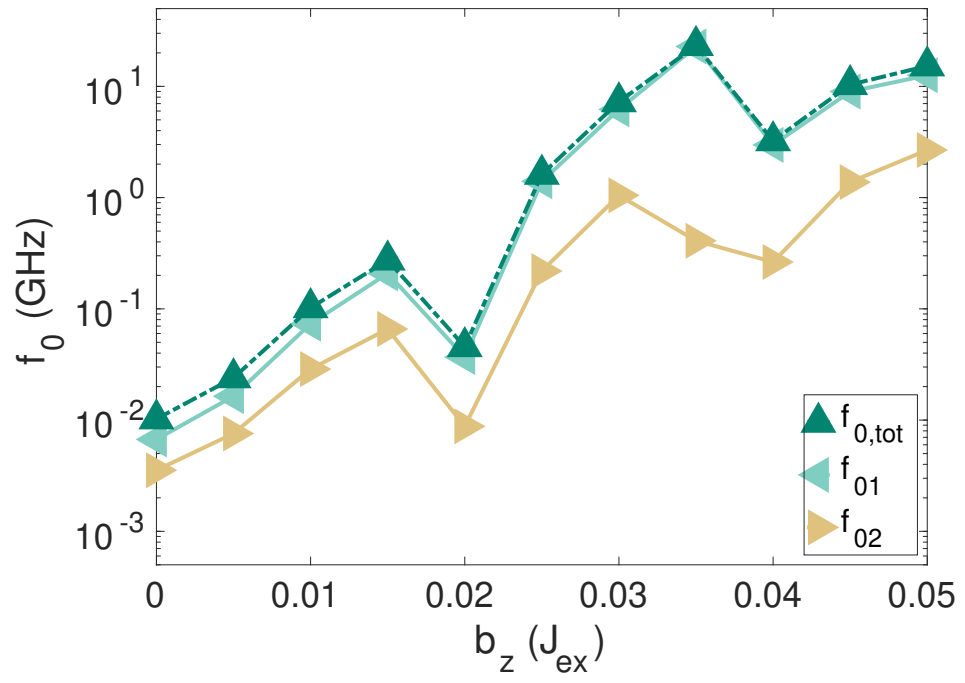

(e)

Figure 4.14: As a function of the reduced destabilizing field $b_{z}$ and for collapse mechanisms $\mathrm{n}^{\circ} 1$ and 2 , we show the following quantities. (a) Internal energy barriers. They both decrease smoothly with increasing field. We find $\Delta E_{1} \lesssim \Delta E_{2}$. (b) Change in configurational entropy when the system goes from states $A$ to $S_{1,2}$ at $T=300 \mathrm{~K}$. We find $\Delta S_{2}<\Delta S_{1}<0$. Since the change is negative in both cases, the entropy decreases at the $\mathrm{SP}$, which is a stabilizing effect. The non-monotonic variations were attributed to lattice effects. (c) Dynamical contribution to the prefactor. For both mechanisms, it decreases only weakly with increasing field, with $\lambda_{+, 1} \lesssim \lambda_{+, 2} \approx \mathrm{THz}$. (d) Total rate of collapse at a temperature such that $\beta \Delta E_{1}=10$ calculated from Eq. (4.2), where $k_{2}(T) \ll k_{1}(T)$ despite the internal energy barriers being almost identical. (e) Attempt frequency for the collapse. Since $\Delta S_{2}<\Delta S_{1}<0$, we have $f_{0,2} \ll f_{0,1}$, which in turns explain why $k_{2}(T) \ll k_{1}(T)$. 


\subsection{Discussion}

In this chapter, we applied Langer's theory to compute annihilation rates of metastable chiral magnetic skyrmions with respect to collapse and escape processes. Two separate mechanisms were identified for the collapse of a single skyrmion. They involve different saddle points, and so each of them is associated with its own rate. We identified the thermally significant modes as the skyrmion's internal modes, while the other modes pertain to collective fluctuations that can be interpreted as spin-wave excitations, which contribute weakly. By changing the underlying symmetry of the DMI, we were able to check that the present results hold for not only Néel skyrmions, but also Bloch skyrmions and antiskyrmions. We found that collapse on a non-magnetic defect yields the shortest lifetimes, with $\tau \approx 0.1 \mu$ s at room-temperature. In the absence of defects, collapse mechanisms were found more probable than escape at low temperature, but this trend reverses at room temperature, at which escape processes are more probable. The largest lifetime at $300 \mathrm{~K}$ was found to be $21 \mathrm{~ms}$, and is associated with collapse mechanism $\mathrm{n}^{0} 2$. The presence of a second skyrmion was shown to decrease the probability of collapse. Finally, escape through a flat boundary was found more probable than through a curved boundary.

We used a simple Heisenberg model limited to first-neighbour exchange interactions and no dipole-dipole couplings, but which should nevertheless capture the essential physics behind skyrmion annihilations in ultrathin films. Similar stability studies where the exchange was parametrized by DFT calculations and went beyond the first nearest-neighbour approximation reached very similar conclusions concerning the importance of the prefactor [63, 109]. In systems were dipole-dipole interactions were found to play an important role in the skyrmions' stability, it was also demonstrated that an effective anisotropy is enough to reproduce similar energy barriers [158]. While this is valid in two-dimensional systems, it may however not be the case in three dimensions. Moreover, the effect of dipolar interactions on the attempt frequency has not been studied, since dipolar couplings yield non-sparse matrices which are difficult to diagonalize in large systems.

The important result of this chapter is the strong entropic contribution to skyrmion stability. In the configurations studied here, the configurational entropy decreases between the metastable state and the saddle point, which results in lowered attempt frequencies. This therefore constitutes a stabilizing effect, which we have referred to as entropic narrowing in the saddle region. The strong entropic contribution is primarily associated with the skyrmion's internal modes, and is more pronounced for collapse mechanisms. The aforementioned effect has two main consequences.

i. When considering different types of processes, the rates of annihilation, $k(T)$, are 
not necessarily ordered like the internal energy barriers. A mechanism with a higher energy barrier might turn out to be less probable because it possesses a low attempt frequency. One example is collapse and escape mechanisms at zerofield. In the present case, collapse processes have a lower energy barrier than escape, but also a much lower attempt frequency. As a consequence, escape processes are more probable than collapse at room temperature. Another example is the case of collapse mechanisms $\mathrm{n}^{0} 1$ and 2 . They have almost the same internal energy barriers, but the attempt frequency associated with mechanism $\mathrm{n}^{\circ} 2$ is much lower, so the total rate is dominated by mechanism $\mathrm{n}^{0} 1$.

ii. The attempt frequency, and therefore the rate, displays a strong dependence on external parameters, such as external magnetic field. This was also discussed in Refs. [46, 63, 157].

For these reasons, internal energy barriers are not enough to correctly predict the lifetime of skyrmions, and it is essential to also evaluate a rate prefactor.

In principle, the total rate of annihilation of an isolated skyrmion in a finite-size system is given by the sum of the rates due to collapse and escape through a boundary, $k^{\text {tot }}=k^{\mathrm{col}}+k^{\mathrm{esc}}$. However, the escape rates we compute here through Langer's theory seem to be instantaneous rates. Since they do not depend on the size of the system (see Table A.1), they do not take into account the time it takes for the skyrmion to diffuse to the boundary. In Ref. [63], the skyrmions are decoupled from the lattice in the metastable state, which results in the emergence of translational Goldstone modes, and a system-size dependence of the rate. This is not satisfactory either, since the breaking of the translational invariance would also result in the loss of system-size dependence. The escape rate we compute therefore corresponds to the probability that a skyrmion which is found close to the boundary, and which cannot diffuse back inside the sample, will escape. The Brownian motion performed by an isolated skyrmion under thermal fluctuations was shown to be diffusive, i.e., with a linear time-dependence of the mean square displacement [143],

$$
\left\langle\Delta \mathbf{R}^{2}\right\rangle=\left\langle(\mathbf{R}(t)-\mathbf{R}(t=0))^{2}\right\rangle \sim \mathcal{D}_{\mathrm{sk}} t
$$

where $\mathbf{R}(t)$ is the position of the skyrmion at time $t$, and $\mathcal{D}_{\text {sk }}$ is the diffusion coefficient of the skyrmion as a particle. The diffusion time $\Delta t_{\text {diff }}$ to reach the boundary at a distance $R_{b}$ from the initial skyrmion position should in principle behave as $\Delta t_{\text {diff }} \sim$ $R_{b}^{2} / \mathcal{D}_{\text {sk }}$, so the full probability that the skyrmion may escape through the boundary should also depend on $T, R_{b}^{2}$, etc, without the need for translational invariance. 
Lastly, the results in this chapter were obtained by applying Langer's theory to the problem of skyrmion lifetime. As we have seen in Chapter 3, this theory is based on many assumptions concerning the damping regime, the energy landscape, and the thermodynamical state of the system. Moreover, whenever we are faced with several mechanisms for a single process, as is the case of the skyrmion collapse, we can only assume the mechanisms are independent in order for the rates to add up (e.g., Eq. (4.2)), which may not hold. We are also faced with the existence of higher-order saddle points, and the question of whether they should contribute to the total rate. An alternative approach is therefore required in order to validate the present results. This will be explored in the next chapter. 


\section{Chapter 5}

\section{Skyrmion collapse rate computation via forward flux sampling and comparison with Langer's theory}

\subsection{Introduction}

Generally speaking, the problem of annihilation rates of skyrmions pertains to the realm of rare events. That is, in a direct Langevin dynamics simulation, few - if any annihilation events are observed. That is because the small timesteps [68] required to properly resolve the precessional dynamics of magnetic spin systems entails that direct simulations are, in practice, limited to a few hundred nanoseconds. Since skyrmions are required to be stable on the scale of ten years at room temperature for data storage applications, a better method than brute force simulations is required. In this chapter, we demonstrate the use of a path sampling method for the simulation of rare events, namely the forward flux sampling method, to compute collapse rates of magnetic skyrmions. We compare the obtained results with the ones calculated in Chapter 4 from the application of Langer's theory. The results presented in this chapter are based on an original publication [3].

The chapter is organized as follows. In Sec. 5.2, we begin by exposing the principles of Langevin spin dynamics and its implementation. In Sec. 5.3, we present the forward flux sampling method, which we then apply in Sec. 5.4 to compute collapse rates of isolated skyrmions. The results are compared with the ones previously obtained in Chapter 4 from the application of Langer's theory and are, lastly, discussed in Sec. 5.5 . 


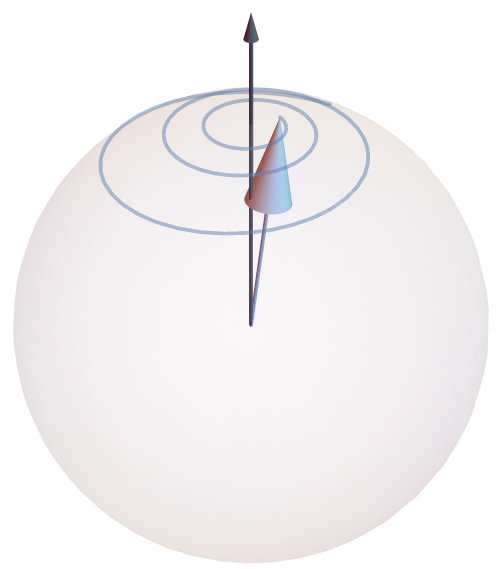

(a)

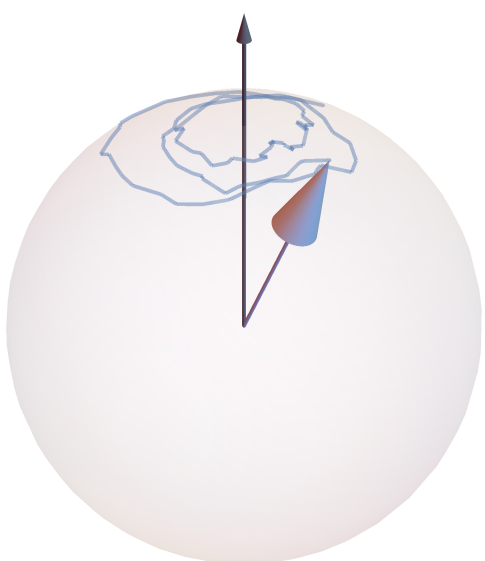

(b)

Figure 5.1: Damped precession of a single magnetic spin (coloured arrow) about the effective magnetic field (dark arrow) (a) at zero temperature (deterministic LLG), (b) at finite temperature (stochastic LLG). In stochastic LLG, the coupling to the environment is modelled by a stochastic fluctuating magnetic field in the form of white noise.

\subsection{Langevin dynamics}

\subsubsection{Principle}

A prerequisite for the implementation of the forward flux sampling method is the ability to properly solve the Brownian dynamics of the magnetic spin system at finite temperature $T$. At zero temperature, the dynamics of each spin $\mathbf{m}_{i}$ is governed by the LLG equation (Eq. (3.46) and Fig. 5.1a), which, at finite temperature, may take the form of a Langevin equation (Eq. (3.4)) through the introduction of a stochastic magnetic field, as given in Ref. [159]

$$
\frac{\mathrm{d} \mathbf{m}_{i}}{\mathrm{~d} \bar{t}}=\frac{1}{\alpha} \mathbf{m}_{i} \times\left[\mathbf{b}_{\mathrm{eff}}^{i}+\mathbf{b}_{\mathrm{fl}}^{i}(\bar{t})\right]-\mathbf{m}_{i} \times\left(\mathbf{m}_{i} \times\left[\mathbf{b}_{\mathrm{eff}}^{i}+\mathbf{b}_{\mathrm{fl}}^{i}(\bar{t})\right]\right) .
$$

Eq. (5.1) is the dimensionless stochastic LLG equation. As in previous chapters, the quantities are in units of the isotropic exchange coupling constant between first nearestneighbours, $J_{1}=J_{\mathrm{ex}}$, such that in the above expression, $\mathbf{b}_{\mathrm{eff}}^{i}$ is the reduced local effective field, as defined in Eq. (3.80), and $\mathbf{b}_{\mathrm{fl}}^{i}$ is a stochastic fluctuating field in the form of white noise, which accounts for fluctuations of the orientation of $\mathbf{m}_{i}$ caused by interactions with microscopic degrees of freedom of the environment such as phonons, conducting electrons, nuclear spins, etc. It is assumed to be Gaussian distributed, with the following statistical properties, in agreement with the fluctuation-dissipation theorem,

$$
\left\langle b_{l}^{i}(\bar{t})\right\rangle=0, \quad\left\langle b_{l}^{i}(\bar{t}) b_{m}^{j}\left(\bar{t}^{\prime}\right)\right\rangle=2 \mathcal{D} \delta_{l m} \delta_{i j} \delta\left(\bar{t}-\bar{t}^{\prime}\right),
$$


where $l, m$ are Cartesian indices, $i, j$ are site indices, and

$$
\mathcal{D}=\frac{\alpha^{2}}{1+\alpha^{2}} \frac{k_{B} T}{J_{1}}
$$

is the reduced diffusion coefficient. $\langle\ldots\rangle$ denotes an average over different realizations of the fluctuating field (see Sec. 3.1.1). The Dirac distribution, $\delta\left(\bar{t}-\bar{t}^{\prime}\right)$, translates the fact that, above a certain temperature, the autocorrelation times of $\mathbf{b}_{\mathrm{fl}}^{i}$ is much shorter than the rotational response of the system. The Kronecker symbol $\delta_{l m}$ comes from the assumption that the different components of $\mathbf{b}_{\mathrm{fl}}^{i}$ are uncorrelated, while $\delta_{i j}$ means that the fluctuating fields at different sites are uncorrelated. The reduced time $\bar{t}$ is linked to physical time $t$ via

$$
t=\frac{M_{s}}{\alpha \gamma_{e} J_{1}} \bar{t} .
$$

In Fig. 5.1b, we show an example of the precession of a single spin about the deterministic effective field obtained by solving Eq. (5.1) at finite temperature. The inclusion of the stochastic fluctuating field results in a stochastic trajectory. As the stochastic field enters in a multiplicative way, an interpretation rule is needed in order to properly define it. This is because the noise has zero correlation time (Eq. (5.2)), so $\mathbf{m}_{i}$ will jump at every time the noise is realized, and so it is not clear which value of $\mathbf{m}_{i}$ should enter on the RHS of Eq. (5.1). Since the white noise is an idealisation of physical noise with short autocorrelation time, the Stratonovich interpretation is the better choice in this case [120]. The Dirac $\delta(t)$ may be approximated by a function $\delta_{\epsilon}(t)$ of very small width $\epsilon$, as [48]

$$
\delta_{\epsilon}(t)= \begin{cases}\frac{1}{\epsilon} & \text { if }-\frac{\epsilon}{2}<t<\frac{\epsilon}{2}, \\ 0 & \text { otherwise. }\end{cases}
$$

One then takes the limit $\epsilon \rightarrow 0$ in the final result.

\subsubsection{Numerical implementation}

\section{The stochastic Heun scheme}

For convenience, we drop the $i$-index associated with magnetic sites, as well as the overbar on the reduced time in this section. The system of stochastic dimensionless LLG equations (Eq. (5.1)) at each spin is cast into a system of Langevin equations (Eq. (3.4)) along each Cartesian axis $l, m, n=\{1,2,3\}$ as [68]

$$
\frac{\mathrm{d} m_{l}}{\mathrm{~d} t}=A_{l}(\boldsymbol{M}, t)+\sum_{n} B_{l n}(\boldsymbol{M}, t) b_{\mathrm{fl}, n}(t),
$$


in which $\boldsymbol{M}=\left[\mathbf{m}_{1}, \ldots \mathbf{m}_{N}\right]$. In the previous expression,

$$
A_{l}=\sum_{n} B_{l n} b_{\mathrm{eff}, n}
$$

is the deterministic right-hand side of Eq. (5.1), and

$$
B_{l n}=\frac{1}{\alpha} \sum_{m} \epsilon_{l m n} m_{m}+\left(\delta_{l n}-m_{l} m_{n}\right),
$$

where $\epsilon_{l m n}$ is the Levi-Civita symbol. We use the stochastic generalization of the deterministic Heun integrator [68], where the value of $m_{l}$ at time $t+d t$ is given by

$$
\begin{aligned}
m_{l}(t+d t)=m_{l}(t) & +\frac{1}{2}\left[A_{l}(\tilde{\boldsymbol{M}}, t+d t)+A_{l}(\boldsymbol{M}, t)\right] d t \\
& +\frac{1}{2} \sum_{n}\left[B_{l n}(\tilde{\boldsymbol{M}}, t+d t)+B_{l n}(\boldsymbol{M}, t)\right] \Delta W_{n}(t) .
\end{aligned}
$$

In the above expression, $d t$ is the integration time step, and $\tilde{\boldsymbol{M}}=\left[\tilde{\mathbf{m}}_{1}, \ldots, \tilde{\mathbf{m}}_{N}\right]$ are the Euler-type supporting values, with each Cartesian component $\tilde{m}_{l, i}=\tilde{m}_{l}$ of $\tilde{\mathbf{m}}_{i}$ expressed as

$$
\tilde{m}_{l}=m_{l}(t)+A_{l}(\boldsymbol{M}, t) d t+\sum_{n} B_{l n}(\boldsymbol{M}, t) \Delta W_{n} .
$$

Lastly,

$$
\Delta W_{n}=\int_{t}^{t+d t} \mathrm{~d} t^{\prime} b_{\mathrm{fl}, n}\left(t^{\prime}\right)
$$

are Gaussian distributed random numbers of first and second moments

$$
\left\langle\Delta W_{n}\right\rangle=0, \quad\left\langle\Delta W_{l} \Delta W_{m}\right\rangle=2 \mathcal{D} d t \delta_{l m},
$$

where $\mathcal{D}$ is defined by Eq. (5.3). The scheme converges to the solution of (5.6) when interpreted in the sense of Stratonovich.

\section{Implementation}

The stochastic Heun integrator for Eq. (5.1) was implemented by the author in $\mathrm{C}++$ following Ref. [68]. At each time step, the Cartesian components of the stochastic field are drawn from a normal distribution defined by Eq. (5.12) using the Mersenne Twister 19937 pseudo-random number generator (MT19937) [160]. 
Chapter 5. Skyrmion collapse rate computation via forward flux sampling and comparison with Langer's theory

\begin{tabular}{ccc}
\hline \hline Temperature $(\mathrm{K})$ & $\left\langle m_{z}\right\rangle_{t, \text { th }}$ & $\left\langle\left|m_{z}\right|\right\rangle_{t, \text { num }}$ \\
\hline 10 & 0.98979 & 0.98978 \\
50 & 0.94268 & 0.94282 \\
200 & 0.71976 & 0.71686 \\
\hline \hline
\end{tabular}

Table 5.1: Theoretical and numerical time average of the component of the magnetization parallel to the anisotropy axis for different temperatures at thermal equilibrium.

\subsubsection{Numerical tests}

\section{Single macrospin}

Based on the tests carried out in Ref. [161], we simulate a single rigid magnetic moment in the presence of an easy-axis anisotropy along $z$, and the following system parameters taken from Ref. $[161]: \mathscr{M}_{s}=1.3 \mathrm{MA} / \mathrm{m} ; \mathscr{K}=6.9 \mathrm{MJ} / \mathrm{m}^{3} ; \alpha=0.1 ; a=1 \mathrm{~nm}$. The reduced time step was set to $\mathrm{d} \bar{t}=0.001$, which corresponds to a physical time step of $\mathrm{d} t=4.55 \times 10^{-15} \mathrm{~s}$.

The first test concerns the correct resolution of the deterministic precessional dynamics of the single-spin system. We initialize the simulation with a tilt angle $\theta=\pi / 4$ with respect to the $z$-axis, and we let the magnetization relax along the anisotropy axis at $T=0 \mathrm{~K}$. The resulting dynamics is shown in Fig. 5.1a. In Fig. 5.2a we plot the time evolution of the Cartesian components of the single spin. The corresponding theoretical precession period is [161]

$$
\mathcal{T}_{\text {th }}=\frac{2 \pi}{\gamma_{e} B_{\text {eff }}}=3.32 \mathrm{ps} .
$$

We measure the time interval between five maxima of $m_{y}$ in Fig. $5.2 \mathrm{a}$ and obtain $\mathcal{T}_{\text {num }}=$ 3.39 ps, which shows good agreement with the theoretical value.

The second test aims to validate the implementation of the stochastic fluctuating field. The theoretical time average for the $z$-component of the magnetization at thermal equilibrium, $\left\langle m_{z}\right\rangle_{t, \text { th }}$, is given in Table 5.1 [161]. We compute $\left\langle m_{z}\right\rangle_{t, \text { num }}$ over a time period $\Delta t=10^{8} d t$, starting from $t_{0}=10^{6} d t$ in order to allow the system to thermalize. Magnetization reversal events may occur, which are not taken into account in the theoretical average. We thus compute $\left\langle\left|m_{z}\right|\right\rangle_{t \text {,num }}$ to reduce the error due to reversals. However, at $T=200 \mathrm{~K}$, reversals are rather frequent, and the numerical average is thus slightly under-evaluated. The results are gathered in the last column of Table 5.1 and plotted in Fig. 5.2b, where the theoretical value is shown as dotted lines. We obtain a good agreement with the theory. 


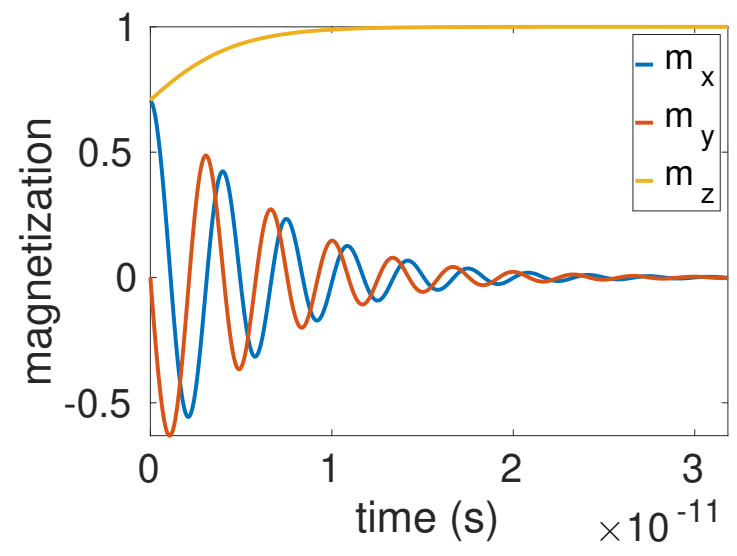

(a)

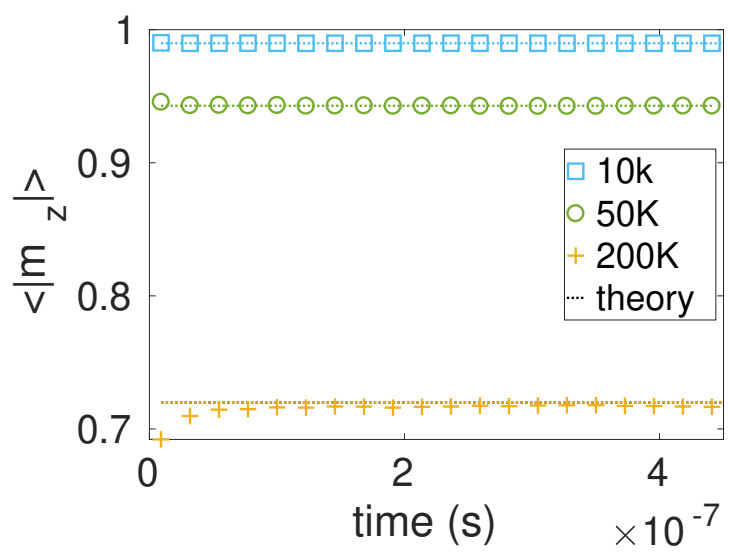

(b)

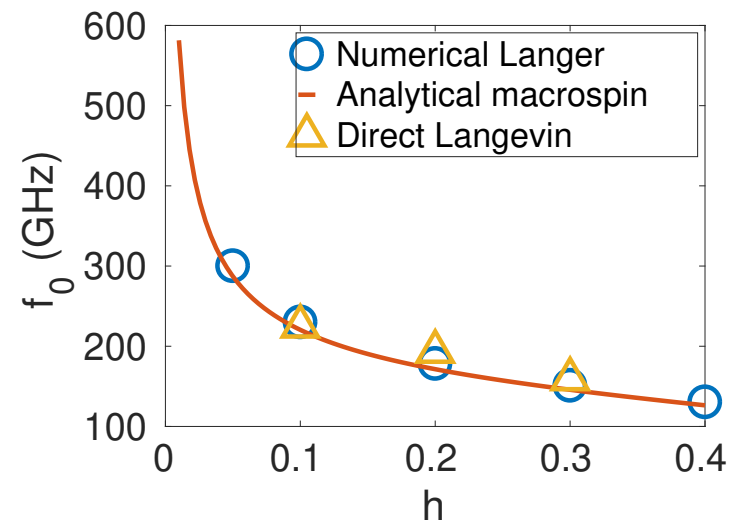

(c)

Figure 5.2: (a) Precessional relaxation of the magnetization for the single rigid magnetic moment at $T=0 \mathrm{~K}$, (b) numerical and theoretical time average value of $\left|m_{z}\right|$ calculated after thermalization for different temperatures, (c) attempt frequency of coherent magnetization reversal in a perpendicular applied field, comparison between the analytical result from Langer's theory for a single macrospin (Eq. (3.94)), and, for $N=7 \times 7$ spins, Langer's theory implementation and direct Langevin simulations at a temperature which verifies $\beta \Delta E=5$.

\section{Magnetization reversal in a perpendicular applied field}

Finally, we go back to the case of coherent magnetization reversal under a perpendicular applied field treated in Sec. 3.5.4. We now aim to compare the attempt frequency obtained from direct Langevin simulations with previous results from Langer's theory. For three arbitrary values of the reduced field $h$, as defined in Eq. (3.93), we set the temperature $T$ such that $\beta \Delta E=5$, where $\Delta E$ is the activation barrier for the reversal. The system parameters are the same as in Sec. 3.5.4, but we now simulate a square system of $N=7 \times 7$ spins. In that case, the numerical result of Langer's theory begins to deviate slightly from the theoretical macrospin case, due to the contribution of collective modes. This can be seen in Fig. 5.2c, where the values of $f_{0}$ computed with the numerical implementation of Langer's theory (shown as blue circles) do not match exactly with the theoretical formula (shown in red). The system is initialized in state $A$ 
with $\phi_{A, i}=\arcsin (h) \forall i$. We launch 400 Langevin simulations and compute an average waiting time for the reversal - i.e., the average time it takes for the system to reach state $B$, which corresponds to $\phi_{B, i}=\pi-\arcsin (h)$. We consider a reversal has happened if $[78]$

$$
\left|\mathbf{M}(t)-\mathbf{M}_{B}\right|<\lambda_{B}
$$

where $\mathbf{M}_{B}=\left[\mathbf{m}_{B, 1}, \ldots \mathbf{m}_{B, N}\right]$, the vertical bars denote the ( $N$-dimensional) Euclidian norm, and $\lambda_{B}$ defines the boundary of region $B . \lambda_{B}$ must be chosen such that it does not impact the value of the reversal rate $k_{A B}$. According to [78], $0<\lambda_{B} \leq 0.5$ is within acceptable values, so we set $\lambda_{B}=0.5$. For higher values of $\lambda_{B}$, U-turn events might be counted as reversals, that is, events where the magnetization precesses around $\mathbf{M}_{B}$ with a small angle, but ends up returning to $A$, in which case the reversal rate gets over-estimated. The attempt frequency $f_{0}$ is linked to the average waiting time $\tau_{A B}$ via

$$
f_{0}=\tau_{A B}^{-1} e^{\beta \Delta E} .
$$

The results of Langevin simulations are shown in Fig. $5.2 \mathrm{c}$ as yellow triangles and agree well with Langer's theory.

\subsection{The forward flux sampling method}

Forward flux sampling (FFS) is a path sampling method for the simulation of rare events in systems with stochastic dynamics. It was initially developed by Allen et al. [74, 75] to simulate rare switching events in biochemical networks. It has, since then, been applied to a wide range of rare event problems [77]. In particular, it was used by Vogler et al. [78] to obtain switching rates in magnetic microstructures. In that context, FFS was shown to be significantly more efficient than direct Langevin simulations, while enabling an exploration of phase space free from prior assumptions. The method generates trajectories between two (meta)stable states $A$ and $B$ in a ratchet-like manner, which makes it well adapted for the simulation of rare to very rare events. Compared to other path sampling methods, it does not require prior knowledge of the phase space probability distribution function, which makes it suitable for both equilibrium and non-equilibrium systems - that is, systems that do not obey detailed balance. For equilibrium systems, detailed balance entails that the stationary phase space probability distribution is given by the Boltzmann distribution (Eq. (3.21)), and the dynamics is time-reversible. In non-equilibrium systems, on the other hand, the probability distribution function is not known a priori. Lastly, FFS requires the rate constant $k_{A B}$ to be time-invariant, which is verified if the transition occurs between two time-invariant steady states $A$ and $B$, and is itself fast compared to $k_{A B}^{-1}$. Following the initial work of 


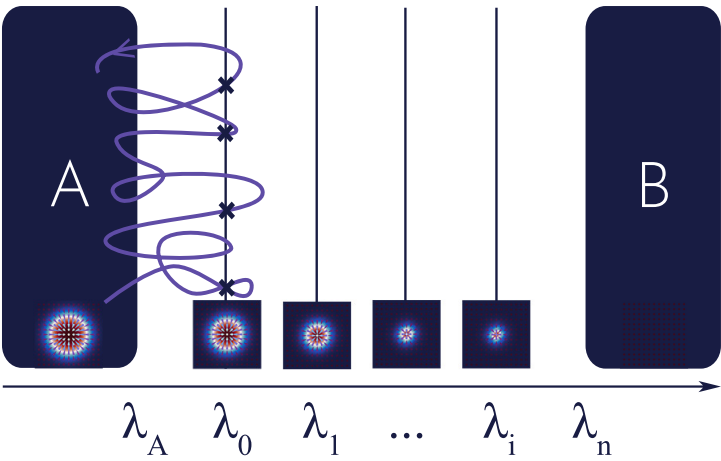

(a)

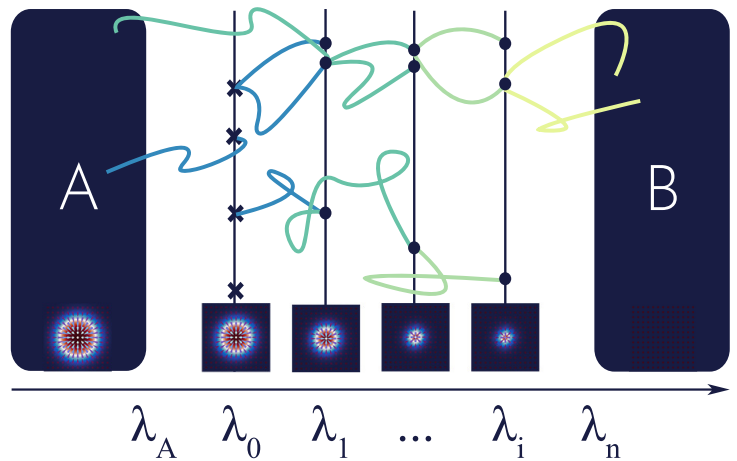

(b)

Figure 5.3: Illustration of the FFS method where interfaces correspond to the isotropic collapse of a skyrmion. (a) During the first simulation starting from state $A$, trajectories (in purple) that exit region $A$ and cross $\lambda_{0}$ are stored (marked as X's). (b) Langevin trial runs are performed at subsequent interfaces. The trajectories that successfully cross the next interface before returning to $A$ are stored (marked as dots) and serve as starting points for the firing of new trial runs at the next interface. For the skyrmion collapse, we chose the order parameter defining the interfaces between states $A$ (metastable skyrmion) and $B$ (ferromagnetic state) as the monotonically decreasing size of the skyrmion.

Allen et al. [74,75], many variants of FFS were developed [77]. In this work, we stick to the original algorithm, known as Direct FFS (DFFS), which we simply refer to as FFS.

\subsubsection{Principle}

The FFS method employs a set of $n(+1)$ non-intersecting interfaces in phase space to sample the transition path ensemble and compute a transition rate. The interfaces $\left\{\lambda_{A}, \lambda_{0}, \ldots \lambda_{n}=\lambda_{B}\right\}$ are defined as isosurfaces of a monotonically varying order parameter, $\lambda(x)=\lambda_{i}$, such that $\lambda_{A}<\lambda_{0}<\ldots<\lambda_{B}$, as illustrated in Fig. 5.3. State $A$ is defined as $\lambda(x)<\lambda_{A}$, and state $B$ as $\lambda(x)>\lambda_{B}$. The other interfaces $\left\{\lambda_{i}\right\}, i=0 \ldots n-1$ must satisfy $\lambda_{i}<\lambda_{i+1} \forall i$. Any trajectory going from $A$ to $B$ must cross all the interfaces at least once. The boundaries of regions $A$ and $B$, i.e., $\lambda_{A}$ and $\lambda_{B}$, require appropriate definitions to ensure that the final rate is independent of the way they are defined, and only the efficiency of the method is affected.

The rate of transition from $A$ to $B$ within the FFS method is expressed as [74]

$$
k_{A B}=\Phi_{A, n}=\Phi_{A, 0} P\left(\lambda_{n} \mid \lambda_{0}\right) .
$$

Eq. (5.16) translates the fact that $\Phi_{A, n}$, which is the flux of trajectories leaving state $A$ and reaching interface $\lambda_{n}=\lambda_{B}$, is given by the product of $\Phi_{A, 0}-$ the flux of trajectories leaving $A$ and crossing the first interface $\lambda_{0}$, and the probability $P\left(\lambda_{n} \mid \lambda_{0}\right)$ that a trajec- 
tory that crosses $\lambda_{0}$ coming from $A$ will subsequently cross $\lambda_{n}$ before returning to $A$. The flux $\Phi_{A, 0}$ can be computed easily, since trajectories coming from $A$ will frequently cross $\lambda_{0}$. On the other hand, the probability $P_{B}=P\left(\lambda_{n} \mid \lambda_{0}\right)$ will typically be small for rare events. It is made easier to calculate by decomposing it into a product of flux through each interface, that is

$$
P_{B}=P\left(\lambda_{n} \mid \lambda_{0}\right)=\prod_{i=0}^{n-1} P\left(\lambda_{i+1} \mid \lambda_{i}\right) .
$$

The conditional probabilities $P\left(\lambda_{i+1} \mid \lambda_{i}\right)$ correspond to the probability that a trajectory coming from $A$ that crossed interface $\lambda_{i}$ for the first time will cross interface $\lambda_{i+1}$ before returning to $A$. Eq. (5.17) does not involve a Markovian approximation, since $P\left(\lambda_{i+1} \mid \lambda_{i}\right)$ depends on the history of trajectories that reached $\lambda_{i}$. Eqs. (5.16) and (5.17) correspond to an "effective positive flux" formulation of the rate constant. One can note that state $B$ is not required to be a (meta)stable state, and FFS may be used to predict the probability of arbitrary rare fluctuations starting from a stable state $A[162]$. The terms in Eq. (5.16) are computed by firing Langevin trial runs at successive interfaces. The protocol is illustrated in Fig. 5.3 and detailed below.

First, a single Langevin simulation is started in state $A$. This initial simulation is used to generate a collection of $N_{0}$ configurations corresponding to crossings of $\lambda_{0}$, as well as to compute a value of $\Phi_{A, 0}$. Following an equilibration period, each time the system successfully exits region $A$ and crosses the next interface $\lambda_{0}$, its configuration is stored. This is illustrated in Fig. 5.3a, where the system trajectory is shown in purple, and stored configurations are marked as X's. The simulation ends after $N_{0}$ crossing configurations have been stored at $\lambda_{0}$, and the flux of trajectories out of state $A$ in Eq. (5.16) is obtained as $\Phi_{A, 0}=N_{0} / \Delta t_{\text {sim }}$, in which $\Delta t_{\text {sim }}$ is the total simulated time. Note that $\Delta t_{\text {sim }}$ should not include the time the system may have spent in the $B$-basin.

After that, a configuration stored at $\lambda_{0}$ is selected at random, and used as a starting point for a new simulation. That new simulation ends when the trajectory either crosses interface $\lambda_{1}$, in which case the crossing configuration is stored, or the system returns to $A$. This is shown in Fig. 5.3b, where trajectories starting from $\lambda_{0}$ are shown in blue. If they cross the next interface, the stored configuration is marked as a dot. To obtain a flux through interface $\lambda_{1}$, this procedure is repeated $M_{0}$ times. If $N_{0}^{s}$ trajectories successfully crossed $\lambda_{1}$, we can compute $P\left(\lambda_{1} \mid \lambda_{0}\right)=N_{0}^{s} / M_{0}$, where $M_{0}$ corresponds to the number of trial runs started at interface $\lambda_{0}$. One then proceeds analogously at subsequent interfaces, until $\lambda_{n}$ is reached. This results in the system being driven in a ratchet-like manner from the initial to the final state, without imposing any bias on the microscopic dynamics. This is achieved by "capitalizing" on fluctuations in the 
direction of the order parameter. The FFS run terminates successfully if at least one trajectory reached state $B$, otherwise the interface definitions need to be improved. A collection of transition paths may be reconstructed a posteriori from the connectivity history of all the trial runs. Note that the obtained transition paths are branched, since many paths may start from a single configuration at $\lambda_{0}$.

\subsubsection{Error estimation and optimization}

\section{Error estimation}

The FFS method involves many adjustable parameters: the number and position of the interfaces, $\left\{\lambda_{i}\right\}$, the definition of the order parameter, $x$, the number of trial runs fired at each interface, $\left\{M_{i}\right\}$, and the number of configurations stored at the first interface, $N_{0}$. The computed rate constant should, in principle, not depend on these parameters, and only affect the efficiency of the method [77]. In order to estimate the efficiency and the error of a given FFS run, a relative variance of the rate, $\mathcal{V}$, was derived in Ref. [76]. $\mathcal{V}$ is defined as the variance $V\left[k_{A B}\right]$ in the estimated value of the rate constant, $k_{A B}$, per starting point at $\lambda_{0}$, divided by the square of the expectation value of the rate:

$$
\mathcal{V}=N_{0} \frac{V\left[k_{A B}\right]}{k_{A B}^{2}} \approx N_{0} \frac{\Phi_{A, 0}^{2} V\left[P_{B}\right]}{\left(\Phi_{A, 0} P_{B}\right)^{2}}=N_{0} \frac{V\left[P_{B}\right]}{P_{B}^{2}} .
$$

In the previous expression, we have used the general formula

$$
V[a x]=a^{2} V[x],
$$

where $a$ is constant. In what follows, let $p_{i} \equiv P\left(\lambda_{i+1} \mid \lambda_{i}\right), q_{i} \equiv 1-p_{i}$, and $k_{i} \equiv M_{i} / N_{0}$. To obtain an expression for the variance of $P_{B}$, we use the following propagation formula:

$$
V\left[f\left\{x_{i}\right\}\right]=\sum_{i=1}^{n}\left(\frac{\partial f}{\partial x_{i}}\right)^{2} V\left[x_{i}\right]
$$

where $f\left\{x_{i}\right\}$ is a function of $n$ uncorrelated variables $\left(x_{1}, x_{2}, \ldots x_{n}\right)$, and the partial derivatives are evaluated with all variables at their mean values. We identify

$$
f\left\{p_{i}\right\}=\prod_{i=0}^{n-1} p_{i}=P_{B},
$$

and, by noting that

$$
\frac{\partial \prod_{j=0}^{n-1} p_{j}}{\partial p_{i}}=\frac{P_{B}}{p_{i}}
$$


we obtain

$$
V\left[P_{B}\right]=V\left[\prod_{i=0}^{n-1} p_{i}\right]=P_{B}^{2} \sum_{i=0}^{n-1} \frac{V\left[p_{i}\right]}{p_{i}^{2}} .
$$

If we launch $M_{i}$ trial runs at interface $i$, and $N_{i}^{s}$ are successful in reaching the next interface, then, by using Eq. (5.19) once more,

$$
V\left[p_{i}\right]=V\left[\frac{N_{i}^{s}}{M_{i}}\right]=\frac{V\left[N_{i}^{s}\right]}{M_{i}^{2}} .
$$

According to [76], $N_{i}^{s}$ is binomially distributed, and so $V\left[N_{i}^{s}\right]=M_{i} p_{i} q_{i}$. By inserting the previous expression and Eq. (5.24) into (5.23), we obtain

$$
V\left[P_{B}\right]=\frac{P_{B}^{2}}{N_{0}} \sum_{i=0}^{n-1} \frac{q_{i}}{p_{i} k_{i}},
$$

and from Eq. (5.18),

$$
\mathcal{V}=\sum_{i=0}^{n-1} \frac{q_{i}}{p_{i} k_{i}} .
$$

Eq. (5.26) holds as long as $M_{i}$ is large enough that there is always at least one trial run that reaches the next interface. $\mathcal{V}$ is assumed to arise only from the trial runs for the computation of $P_{B}$ (Eq. (5.17)), and not from the computation of the initial flux $\Phi_{A, 0}$. This is justified for a large enough flux out of region $A$ and a long enough simulation time in the $A$ basin. In practice, we must chose $N_{0}$ so that the value of $\Phi_{A, 0}$, and subsequent value of $k_{A B}$, do not strongly depend on it. Finally, we note that Eq. (5.26) was obtained by assuming that the number of successful trial runs at different interfaces are uncorrelated. In other words, the probability $p_{i}$ of a successful trial run at $\lambda_{i}$ does not affect the probability $p_{i+1}$ of a successful trial run at $\lambda_{i+1}$. In reality, this assumption may not hold for highly correlated systems, and/or if the spacing between interfaces is small.

Statistical errors on the obtained transition rates can be extracted from the relative variance (Eq. (5.26)), or by repeating several FFS runs. In the former case, the error on $k_{A B}$ may be estimated as [76, 77],

$$
\sigma_{k}=k_{A B} \sqrt{\frac{\mathcal{V}}{N_{0}}} .
$$


The statistical error on the attempt frequency $f_{0}$ is easily derived from Eq. (5.27), and we obtain

$$
\sigma_{f_{0}}=f_{0} \sqrt{\frac{\mathcal{V}}{N_{0}}} .
$$

Note that [78] shows that the standard deviation of the attempt frequency obtained by repeating five separate FFS simulations tends to be much bigger than the one computed with Eq. (5.28), as least in the case where $N_{0}=100$ was used. Apart from estimating a standard deviation, the relative variance is also useful to evaluate the computational efficiency of the method, and compare it with that of "brute force" direct Langevin simulations [76].

\section{Optimization}

Some iterative procedures were designed by Borrero and Escobedo [163 to optimize the parameters of FFS. The principle consists in minimizing the variance $V\left[P_{B}\right]$ under the constraint that the total transition probability $P_{B}$ remains constant. Varying Eq. (5.25) with a Lagrange multiplier yields the condition [163]

$$
M_{i} p_{i}=\mathrm{cst}
$$

that is to say, a constant flux of partial trajectories through all interfaces is the optimal configuration to minimize the variance of the rate. From there, two main approaches are possible:

- optimizing the number of trial runs $\left\{M_{i}\right\}$ while keeping the interfaces fixed;

- optimizing the interface placement $\left\{\lambda_{i}\right\}$ at fixed $\left\{M_{i}\right\}$.

In this work, we have maintained a constant number of trial runs per interface, $M_{i}=M_{0}$ $\forall i$, but, when judicious, we optimized the placement of interfaces. The principle is explained in what follows.

After choosing a fixed value of $M_{i}$ through all interfaces, the condition (5.29) implies that

$$
p_{i}=\mathrm{cst}=\left(P_{B}\right)^{\frac{1}{n}} .
$$

The value of the transition probabilities $\left\{p_{i}\right\}$ can be adjusted by changing the placement of the interfaces $\left\{\lambda_{i}\right\}$. To do so, an interpolation function is needed in order to establish a correspondence between the $\left\{p_{i}\right\}$ and the $\left\{\lambda_{i}\right\}$. A possible choice of such a function is $[163$

$$
f\left(\lambda_{i}\right)=\frac{\sum_{j=0}^{i-1} \ln p_{j}}{\sum_{j=0}^{n-1} \ln p_{j}},
$$


which reduces to

$$
f\left(\lambda_{i}\right)=\frac{i}{n}
$$

when $\left\{p_{i}\right\}=$ cst. The optimization procedure is as follows:

- perform a run of FFS with a non-optimized set of interfaces $\left\{\lambda_{i}\right\}$ and compute $\left\{f\left(\lambda_{i}\right)\right\}$ from Eq. (5.31);

- compute the optimum values of $\left\{f\left(\lambda_{i}\right)\right\}$ according to Eq. (5.32);

- invert the interpolation function to determine the new set of optimized $\left\{\lambda_{i}\right\}$;

- iterate the procedure if necessary.

\subsubsection{Numerical implementation}

\section{Implementation of parallel trial runs}

Although a linear implementation of FFS is straightforward, the method is more efficient when many trial runs at a given interface $\lambda_{i}$ can be performed simultaneously. This requires the simulation to wait for all trial runs started at interface $\lambda_{i}$ to terminate, in order to launch the next series of trial runs at interface $\lambda_{i+1}$.

All the code was written by the author, unless stated otherwise. The core code was implemented in $\mathrm{C}++$ and performs a given trial run $k$, where $k=1 \ldots M_{i}$, at interface $\lambda_{i}$, until the system trajectory either returns to $A$ or crosses $\lambda_{i+1}$. In the latter case, the configuration is stored in a file. The next layer is managed by a Bash script. The script stores the indices and the number $N_{i}^{s}$ of crossing configurations saved from the trial runs fired at interface $\lambda_{i-1}$, and selects one at random to launch a trial run at interface $\lambda_{i}$. If $\lambda_{i}=\lambda_{0}$, it simply selects a random configuration in $\left[1, N_{0}\right]$. The FFS simulations were performed across several nodes - typically 10 nodes, with 16 tasks per node and two tasks per core, for a total of 320 simultaneous tasks - on the Vienna Scientific Cluster (VSC). Running parallel trial runs on multiple nodes requires the use of MPI (Message Parsing Interface) to allow the trial runs to wait for each other before moving on to the next interface. An MPI for Python script initially written by Dr. Christoph Vogler was modified by the author and used in that effect. The Python script calls the Bash script with some given parameters and manages the global FFS run over all interfaces and all trial runs on the VSC.

\section{Numerical test}

Once more, we test the implementation against the rate of coherent magnetization reversal in a perpendicular field (Fig. 3.6a). We reproduce the low damping case of 
Ref. [78], for which we use the same parameters describing a single-macrospin particle with $\alpha=0.002, \mathscr{K}=3 \mathrm{MJ} / \mathrm{m}^{3}, a=1 \mathrm{~nm}, N=1, \mathscr{M}_{s}=0.5 \mu_{0}^{-1} \mathrm{~A} / \mathrm{m}$. Region $A$ and $B$ are defined according to Eq. (5.14), with $\lambda_{A, B}=0.45$. The other interfaces are defined as hyperplanes in the space of configurations in the following way. We use the collection of images along the MEP, $\left[\mathbf{M}^{1} \ldots \mathbf{M}^{Q}\right]$, previously found with the GNEB scheme. Note that we may skip the images that lie past the saddle point, since the flux of trajectories starting from the saddle point that subsequently reach state $B$ is high. Therefore, if the climbing image is at the saddle point, we can restrict ourselves to $\left[\mathbf{M}^{1} \ldots \mathbf{M}^{v_{C I}}, \mathbf{M}^{Q}\right]$, where $\mathbf{M}^{Q}=\mathbf{M}^{B}$. At each spin $i$, and each image $v$, we define the local vector to the previous configuration,

$$
\mathbf{n}_{i}^{v}=\mathbf{m}_{i}^{v}-\mathbf{m}_{i}^{v-1} .
$$

Interface $v$ is defined as the hyperplane normal to $\mathbf{N}^{v}=\left[\mathbf{n}_{1}^{v} \ldots \mathbf{n}_{N}^{v}\right]$ that passes through $\mathbf{M}^{v}$. At each time step, we compute the dot product

$$
\mathbf{N}^{v} \cdot\left[\mathbf{M}(t)-\mathbf{M}^{v}\right] .
$$

We consider that interface $v$ has been crossed if the dot product in Eq. (5.3) has changed its sign. Note that, for the problem of magnetization reversal, one may also use Euclidian norm definitions (Eq. (5.14)) for all interfaces [79]. We use eight interfaces, $N_{0}=100$, and $M_{i}=M_{0}=3840$ at all interfaces. The temperature in the Langevin trial runs is chosen such that $\beta \Delta E=14$. For a reduced field $h=0.1$ (Eq. (3.93)), we obtain an initial flux of $\Phi_{A, 0}=1.06 \mathrm{GHz}$, and an attempt frequency of $f_{0}=147.5 \mathrm{GHz}$, with a relative variance $\mathcal{V}=0.48$. This matches the result from Ref. [78] and the analytical formula derived in [164].

\subsection{Application to skyrmion collapse and comparison with Langer's theory}

\subsubsection{Interface definition}

We aim to compare the collapse rates of an isolated skyrmion previously calculated via Langer's theory, with the rates computed via FFS simulations. A natural choice of interfaces for the skyrmion collapse is the decreasing size of the skyrmion, where state $A$ is the equilibrium skyrmion size, and state $B$ - corresponding to the ferromagnetic state - is associated to a zero-size. Arbitrarily, we consider that magnetic sites $\mathbf{m}_{i}$ that satisfy $m_{z, i} \leq 0$ are part of the skyrmion, and we define the order-parameter $x$ as the 
(integer) number of sites inside the skyrmion,

$$
x=\sum_{\substack{i=1 \\ \mathbf{m}_{z, i} \leq 0}}^{N} 1 .
$$

The interfaces $\lambda_{i}=\lambda(x)$ are isosurfaces of $x$ (Fig. 5.3). We set $\lambda_{A}=\lambda\left(x_{A}\right)$, where $x_{A}$ is the number of sites in the metastable skyrmion, and $\lambda_{n}=\lambda_{B}=\lambda(x=0)$. From there, we may choose, for instance, the first interface to be $\lambda_{0}=\lambda\left(x_{A}-3\right)$. This ensures that the first simulation out of the $A$ basin will span over a long enough simulated time. The rest of the interfaces may be evenly spaced out between $\lambda_{0}$ and $\lambda_{B}$. However, the efficiency of FFS is increased if the flux of partial trajectories remains as constant as possible through all interfaces. Therefore, we usually saturate the interfaces (one interface every integer value of $x$ ) in the region preceeding the saddle point, since the flux typically tends to drop in that region. The rest of the interfaces can be more spaced out. After a first run of FFS has terminated, it can be helpful to run the interface optimization procedure described in Sec 5.3.2. However, this scheme is designed for a continuous order parameter, and is not as effective if the order parameter only takes integer values. Although we did not implement it, a good solution in this case would be to also optimize the number of trial runs per interface $\left\{M_{i}\right\}$ while maintaining a constant set of $\left\{\lambda_{i}\right\}$. Lastly, for higher field values, at which the metastable skyrmion is small, we simply saturate the interfaces between $\lambda_{0}$ and $\lambda_{B}$.

The number of stored configurations at $\lambda_{0}$ was chosen to be $N_{0}=1000$, or 1500 for $b_{z}=0$. Since the trajectories are branched, we have found that the exhaustiveness of this initial sampling is crucial. If not enough crossing configurations are sampled, the fluxes of trajectories through subsequent interfaces are also not accurate. As for the number of trial runs per interface, it is chosen constant and equal to $M_{0}$ at all interfaces, and is iteratively increased until the variance (Eq. (5.26)) verifies $\mathcal{V} \lesssim 1$. In some cases, the variance was decreased also by increasing the number of interfaces ( $p_{i}$ increases). In the end, the number of interfaces spans from 34 at zero-field, to 14 for $b_{z}=0.05$. For each value of the reduced field at $\beta \Delta E_{1}=10$, Table 5.2 shows the corresponding number of interfaces, as well as the values of the absolute temperature of the trial runs, the number of trial runs performed at each interface, $M_{0}$, the number of configurations stored at the first interface, $N_{0}$, and the relative variance of the rate, $\mathcal{V}$.

\subsubsection{Results}

For each value of the reduced field, we compute a total collapse rate $k_{A B}$ through forward flux sampling, and an attempt frequency is recovered by using the value of $\Delta \bar{E}$ 


\begin{tabular}{c|cccccc}
\hline \hline$b_{z}\left(J_{\text {ex }}\right)$ & 0.000 & 0.005 & 0.010 & 0.015 & 0.020 & 0.025 \\
\hline Number of interfaces & 34 & 32 & 24 & 22 & 20 & 17 \\
$\Delta E_{1} / 10 k_{B}(\mathrm{~K})$ & 327.5 & 283.2 & 245.1 & 210.0 & 182.1 & 156.1 \\
$N_{0}$ & 1500 & 1000 & 1000 & 1000 & 1000 & 1000 \\
$M_{0}$ & 34560 & 27520 & 37760 & 38080 & 39680 & 25920 \\
$\mathcal{V}$ & 1.01 & 0.86 & 0.76 & 0.70 & 0.58 & 1.00 \\
\hline \hline
\end{tabular}

\begin{tabular}{c|ccccc}
\hline \hline$b_{z}\left(J_{\text {ex }}\right)$ & 0.030 & 0.035 & 0.040 & 0.045 & 0.050 \\
\hline Number of interfaces & 17 & 17 & 14 & 14 & 14 \\
$\Delta E_{1} / 10 k_{B}(\mathrm{~K})$ & 132.8 & 111.8 & 92.9 & 76.2 & 61.2 \\
$N_{0}$ & 1000 & 1000 & 1000 & 1000 & 1000 \\
$M_{0}$ & 39680 & 39680 & 39680 & 39680 & 39680 \\
$\mathcal{V}$ & 0.66 & 0.73 & 0.73 & 0.84 & 0.85 \\
\hline \hline
\end{tabular}

Table 5.2: For the FFS runs at $\beta \Delta E_{1}=10$ and for each value of the reduced field $b_{z}$, we give: the number of interfaces, the absolute temperature, the number of configurations stored at the first interface, $N_{0}$, the number of trial runs per interface, $M_{0}$, and the relative variance of the rate, $\mathcal{V}$.

\begin{tabular}{c|cccccccccccc}
\hline \hline$i$ & 1 & 2 & 3 & 4 & 5 & 6 & 7 & 8 & 9 & 10 & 11 & 12 \\
$x$ & 26 & 23 & 20 & 19 & 18 & 17 & 16 & 15 & 14 & 13 & 12 & 11 \\
$p_{i-1}$ & 0.18 & 0.27 & 0.35 & 0.70 & 0.68 & 0.67 & 0.65 & 0.63 & 0.59 & 0.57 & 0.54 & 0.52 \\
\hline \hline
\end{tabular}

\begin{tabular}{c|ccccccccccc}
\hline \hline$i$ & 13 & 14 & 15 & 16 & 17 & 18 & 19 & 20 & 21 & 22 & 23 \\
$x$ & 10 & 9 & 8 & 7 & 6 & 5 & 4 & 3 & 2 & 1 & 0 \\
$p_{i-1}$ & 0.49 & 0.48 & 0.43 & 0.39 & 0.39 & 0.37 & 0.40 & 0.28 & 0.53 & 0.77 & 0.99 \\
\hline \hline
\end{tabular}

Table 5.3: For $b_{z}=0.010$ and $\beta \Delta E_{1}=10$, we give the interface index, $i$, the number of sites inside the skyrmion, $x$, and the flux $p_{i-1}=P\left(\lambda_{i} \mid \lambda_{i-1}\right)$ through $\lambda_{i}$. 


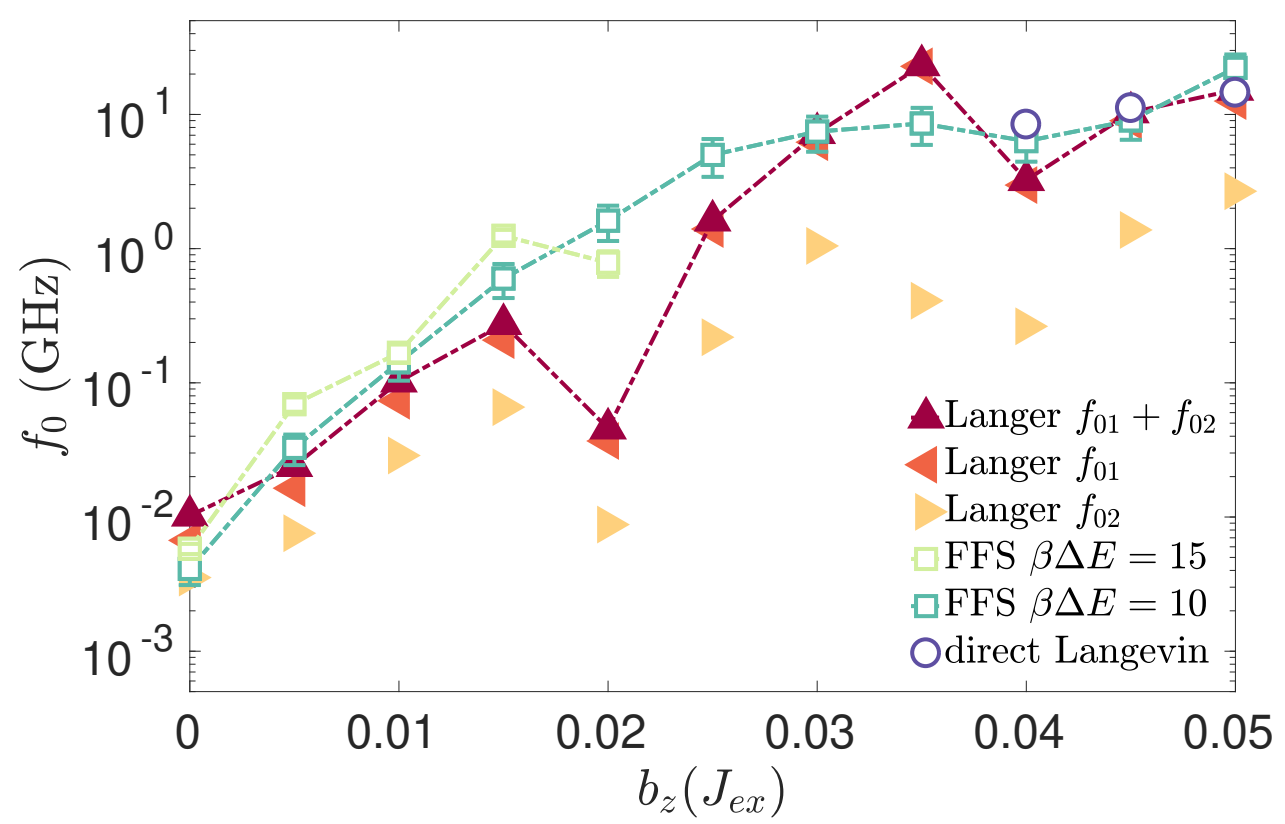

(a)

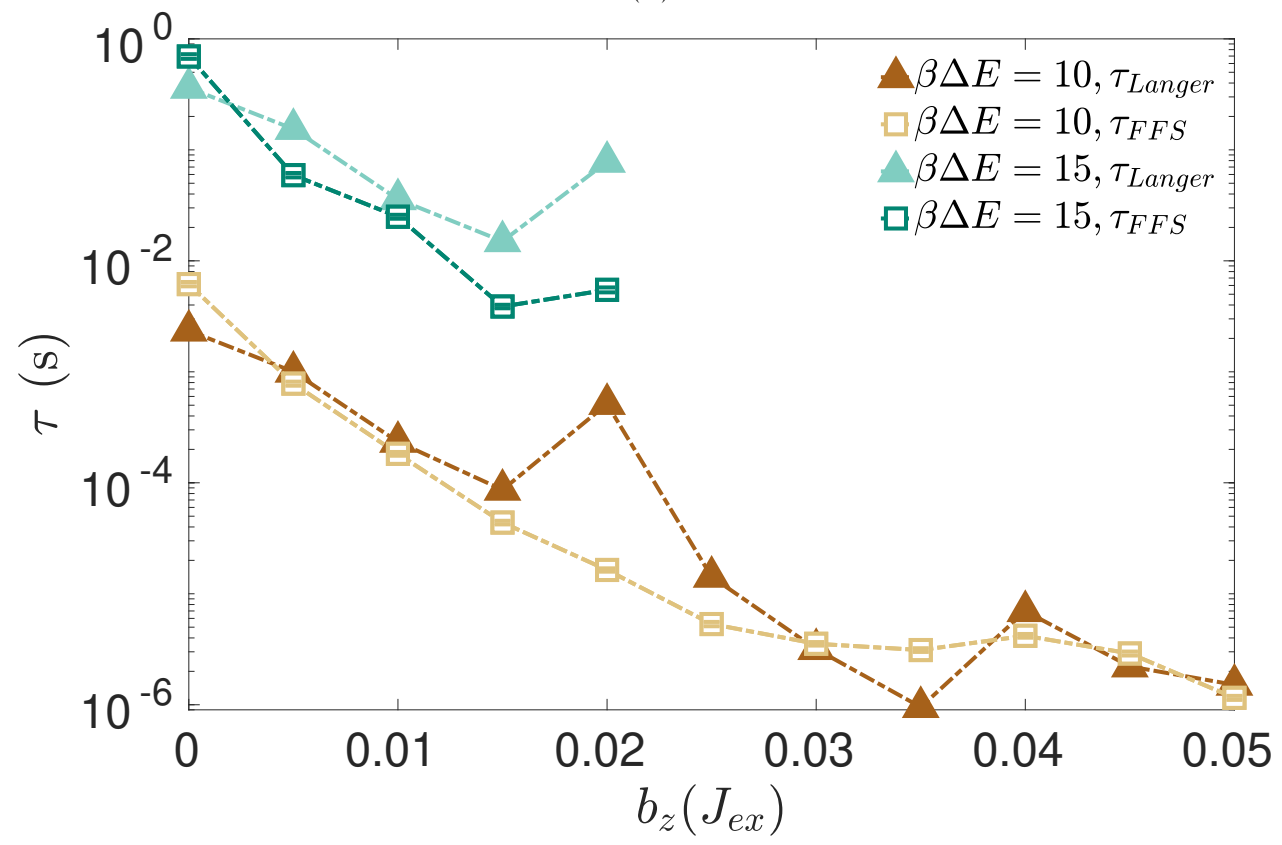

(b)

Figure 5.4: We show the following quantities as a function of the reduced destabilizing applied field $b_{z}$ : (a) Attempt frequency $f_{0}$ for the skyrmion collapse obtained via Langer's theory calculations, FFS simulations, and direct Langevin simulations at 300 $\mathrm{K}$, (b) skyrmion lifetime against collapse, $\tau=k_{A B}^{-1}$, obtained via Langer's theory and FFS simulations. The results from Langer's theory are the ones presented in Chapter 4 . The errorbars on FFS calculations are respectively given by Eqs. (a) (5.37) for the attempt frequency and (b) (5.27) for the average lifetime. Note that whenever the errorbars are smaller than the size of the square marker, they are represented as two unconnected parallel lines. 
(a)
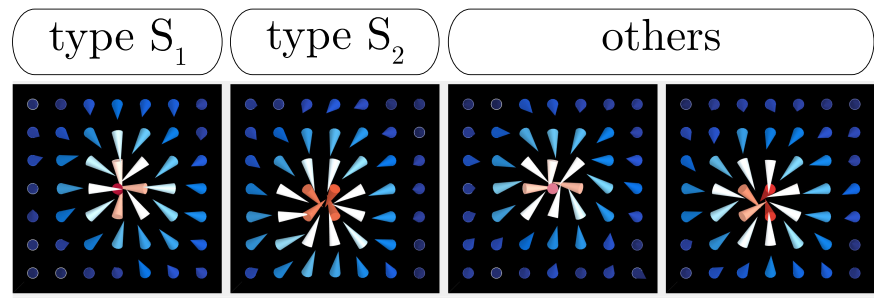

(b)
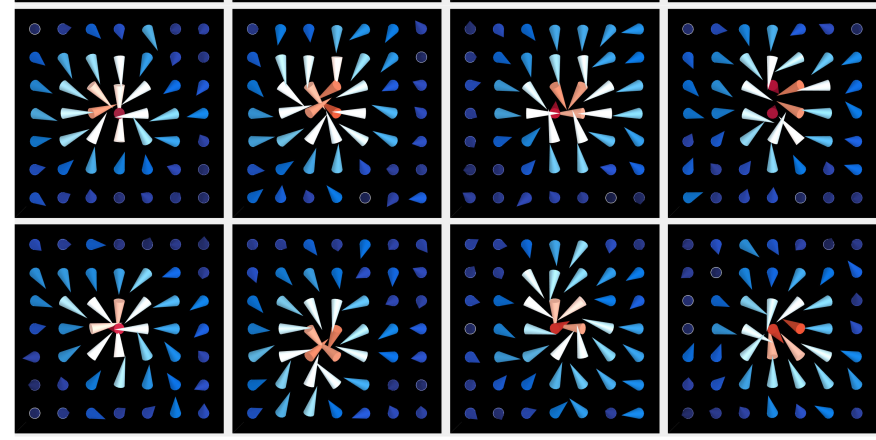

(c)
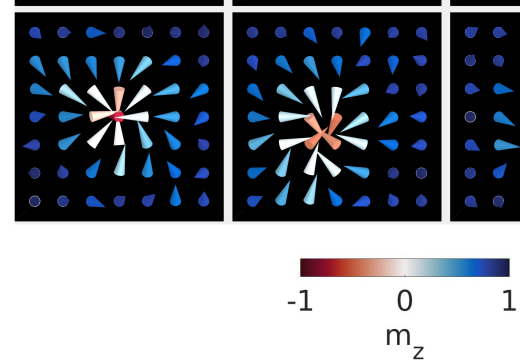

Figure 5.5: Examples of (zoomed) spin configurations from FFS stored at the interface(s) at which the order-parameter equates that of a saddle point (a) at interface $\lambda_{9}=\lambda(x=4)$, for $b_{z}=0.05$ and $T=61 \mathrm{~K}\left(\beta \Delta E_{1}=10\right),(\mathrm{b}, \mathrm{c})$ for $b_{z}=0$ and $T=218$ $\mathrm{K}\left(\beta \Delta E_{1}=15\right)$, at interface (b) $\lambda_{28}=\lambda(x=5)$, and (c) $\lambda_{29}=\lambda(x=4)$.

(Eq. (4.3)). Throughout each FFS run, we maintain a temperature $T$ such that $\beta \Delta E_{1}=$ 10 or 15 , so that Langer's theory holds [47]. For $b_{z}=0.010$, we get $\Phi_{A, 0}=104.39$ $\mathrm{GHz}$ with $\lambda_{0}=\lambda(x=29)$. For this particular field value, FFS matches really well with Langer's theory, and so we give in Table 5.3 the values of the order parameter $x$, and the corresponding flux $p_{i-1}$ through each interface $\lambda_{i}$, in the case where $\beta \Delta E_{1}=10$. The results from both FFS and Langer's theory are shown in Fig. 5.4a for the attempt frequency $f_{0}$, and the skyrmion lifetime in an infinite system, $\tau_{A B}=k_{A B}^{-1}$, is plotted in Fig. 5.4b. The standard deviation on the lifetime is derived from Eq. (5.27) and is expressed as

$$
\sigma_{\tau}=\frac{1}{k_{A B}} \sqrt{\frac{\mathcal{V}}{N_{0}}} .
$$

For the attempt frequency, the formula is modified to account for the error on the internal energy barrier in the following way:

$$
\sigma_{f_{0}}=f_{0}\left(\sqrt{\frac{\mathcal{V}}{N_{0}}}+\beta \sigma_{\Delta E}\right),
$$

where $\sigma_{\Delta E}$ is the standard deviation associated with $\Delta \bar{E}$. At higher applied field values, where the attempt frequency is found within the $1-10 \mathrm{GHz}$ range, we also compute a collapse rate via direct Langevin simulations at $300 \mathrm{~K}$. In that case, we proceed simi- 
larly to the FFS method, by counting the number of sites $x$ inside the skyrmion at each time step, as defined in Eq. (5.35). A collapse is recorded when $x=0$. We obtain an average lifetime out of 400 collapses, and once again we use the value of $\Delta \bar{E}$ to recover an attempt frequency.

Overall, we obtain a good agreement between Langer's predictions and FFS. The three data points obtained from direct Langevin simulations match the result of FFS. The prefactors obtained at two different temperatures in the FFS runs agree well, which validates Langer's result that, in the present case, the attempt frequency does not exhibit any significant temperature dependence. Over the range of applied field strengths studied here, FFS confirms the prediction that the prefactor $f_{0}$ varies by four orders of magnitude, which is the result of a strong entropic contribution.

In Fig. 5.5, we plot some examples of stored configurations at interfaces at which the order-parameter is that of a saddle point. We report both $S_{1}$ and $S_{2}$ types of configurations, as well as some other configurations that don't clearly pertain to either category. This occurs for two reasons. Firstly, the crossing configurations correspond to an order parameter which is either equal to, or smaller than that of the saddle point $\left(x \leq x_{\mathrm{SP}}\right)$. Even in cases where $x=x_{\mathrm{SP}}$, this does not imply that the configuration is indeed a saddle point. Secondly, the saddle point is the lowest energy configuration at the barrier top. Under the effect of thermal fluctuations, the system does not usually cross the barrier exactly at the SP, but deviates from it by a more or less small amount. Note that Langer's theory is in principle valid only for small deviations.

\subsection{Discussion}

In this chapter, we have compared the rate of collapse of an isolated skyrmion computed via Langer's theory and forward flux sampling simulations. Overall, both methods agree well, thus, in general, the choice of either method should yield the correct order of magnitude for the skyrmion lifetime. Therefore, we can confirm the result previously obtained from Langer's theory that the stability of skyrmions is subject to a strong entropic contribution, and that internal energy barriers are not enough to properly predict their lifetime.

For some values of the applied field (most notably, $b_{z}=0.02$ ), the attempt frequency predicted by Langer shows a discrepancy of one or two orders of magnitude with the FFS result. In general, since the application of Langer's theory is bound by many hypotheses concerning the thermal fluctuations, the energy landscape, and the lack of 
coupling between different mechanisms, the result of FFS should be more trustworthy. On the other hand, FFS is less computationally efficient and less systematic in its application, because the interfaces need to be adjusted to reflect different sets of parameters. The computational cost of both Langer and FFS will obviously increase with the system size, and if non-local dipolar couplings are included, but the cost of FFS will in principle increase faster. For different types of processes, such as escape processes, the nature of the order parameter should be modified accordingly.

As far as we are aware, the results in this chapter constitute the first comparison between forward flux sampling and Langer's theory in a complex, nontrivial magnetic system that cannot easily be treated analytically (i.e., beyond the macrospin approximation). In particular, in addition to the path over the first-order saddle point, $S_{1}$, we included the contribution of a path involving a third-order saddle point, $S_{2}$, to Langer's result in Chapter 4. Multidimensional formulations of reaction rate theory are typically found for a transition path involving a first-order saddle point, and only Ref. ?? proposed an extension of Langer's theory to include higher-order saddle points. Nevertheless, the activation energies associated with $S_{2}$ are almost the same as the ones associated with $S_{1}$, and configurations similar to $S_{2}$ are observed in FFS, so we could not justify neglecting it a priori. Only through the computation of the attempt frequencies in Chapter 4 did it transpire that the mechanism involving $S_{2}$ should not contribute as much as the one involving $S_{1}$, because the attempt frequencies for mechanism $\mathrm{n}^{0} 2$ tend to be much lower (see Fig. 5.4a). This is because the configurational entropy at $S_{2}$ is lower than at $S_{1}$, which implies that $S_{2}$ is less likely to be visited by the system. This result seems to be confirmed by FFS simulations, since the obtained attempt frequencies tends to match Langer's prediction. This is further proof of the importance of the prefactor, because, even though $\Delta E_{1} \approx \Delta E_{2}, k_{1}(T) \gg k_{2}(T)$ (see Fig. 4.14d). We should also note that the energy barriers between $S_{1}$ and $S_{2}$ are likely to be quite low, therefore, in reality, both mechanisms are probably coupled in some way.

Finally, at zero-field and $T=200 \mathrm{~K}$, the skyrmion's average lifetime against collapse approaches one second, which may be encouraging for applications. In multilayers, where the layers are stacked along the $z$-direction, the energy barriers should increase if the spin texture is homogeneous along $z$, thus enhancing stability [105]. Additionally, in $\mathrm{Pd} / \mathrm{Fe}$ bilayers on $\operatorname{Ir}(111)$, frustrated exchange has also been shown to increase the height of the activation barriers against collapse [165]. In the next chapter, we will look at the paths to annihilation of skyrmions under frustrated exchange couplings. 


\section{Chapter 6}

\section{Paths to annihilation of first- and second-order (anti)skyrmions under frustrated exchange}

\subsection{Introduction}

As we have seen in Sec. 2.3.4, skyrmionic solutions can exist in centrosymmetric systems when, for instance, a characteristic lengthscale is introduced via exchange frustration. Systems with frustrated exchange are particularly interesting, as they allow many different kinds of topological defects to coexist. Frustrated exchange was shown to arise from interface effects in $\mathrm{Pd} / \mathrm{Fe} / \mathrm{Ir}(111)$ for fcc stackings of the Pd layer by DFT calculations. This system was also shown to host nanoscale interface skyrmions stabilized primarily by the DMI [33, 43]. The ab initio calculations in Ref. [43] were in good agreement with the different phases of the system reported from SP-STM experiments [33]. More recently, a strong exchange frustration was observed in $\mathrm{Rh} / \mathrm{Fe} / \mathrm{Ir}(111)$, which was found to be a driving force behind the SS ground state [117]. Lastly, during the writing phase of this thesis, strong exchange frustration was reported in $\mathrm{Rh} / \mathrm{Co} / \operatorname{Ir}(111)$ by SP-STM measurements and DFT calculations [118]. In this system, skyrmions of approximately $5 \mathrm{~nm}$ in diameter were observed at zero magnetic field at low temperature in the virgin FM state. The exchange frustration was found to play an important role in their stabilization. These results demonstrate the importance of understanding the effect of exchange frustration on skyrmion stability. The frustrated $J_{1}-J_{2}$ system on the hexagonal lattice was extensively investigated in Ref. [22] and, in general, the frustrated hexagonal lattice is the most commonly studied [23, 26, 43, 139, 165]. Although skyrmions decouple from the lattice in the long wavelength limit, we can note that this is typically not true for saddle point configurations of collapse processes [1, 63]. Collapse mechanisms of skyrmions and antiskyrmions in chiral thin films with frustrated 
exchange were previously investigated [63, 165], and appear similar to the isotropictype of collapse calculated in non-frustrated chiral systems [1, 59, 67, 141, 158, 166]. In $\mathrm{Pd} / \mathrm{Fe} / \mathrm{Ir}(111)$, the energy barriers of isolated skyrmions with respect to isotropic collapse were found to be enhanced by the exchange frustration, compared to a Hamiltonian limited to first nearest-neighbours [165]. A third annihilation mechanism involving the injection of a singularity with opposite topological charge was reported in [67], but the path appears to go over a higher order saddle point. Additionally, this mechanism seems to emerge for larger skyrmion sizes. Lastly, asymmetric paths to collapse similar to the ones we present in this chapter were very recently reported via GNEB calculations for first-order skyrmion solutions on the frustrated hexagonal lattice [118,167].

In this chapter, we look at paths to annihilation of first- and second-order skyrmions and antiskyrmions on the frustrated $J_{1}-J_{2}-J_{3}$ square lattice previously studied by Lin et al. [25], which we presented in Section 2.3.4. We relax skyrmionic solutions spanning over only a few sites in diameter that, once again, do not exhibit translational invariance on the lattice, and instead experience pinning at particular lattice points. By using the GNEB method, we compute a new type of asymmetric path to collapse. A weak interfacial DMI is added to break the invariance with respect to the rotation of helicity (i.e. the "spin" degree of freedom, where "spin" refers to the spin of the skyrmion as a particle [25]), as well as the degeneracy between skyrmions and antiskyrmions. The results presented in this chapter were initially published in Ref. [2].

The chapter is organized as follows. In Sec. 6.2, we give regions of existence of metastable first- and second-order skyrmions and antiskyrmions in parameter space. In Sec 6.3, we present different mechanisms by which they annihilate, which we then validate by means of direct Langevin simulations in Sec. 6.4. We subsequently compute attempt frequencies associated with the different mechanisms by applying Langer's theory in Sec 6.5, and the average lifetimes of the stuctures are discussed. Finally, the results are discussed in Sec. 6.6.

\subsection{Coexistence of skyrmion and antiskyrmion solutions}

The system we study is similar to the one described in Chapter 4, but the isotropic exchange is now extended to the third shell of neighbours, with the following reduced coupling parameters: $\left(j_{1}, j_{2}, j_{3}\right)=(1,-0.35,-0.15)[25]$. The reduced perpendicular uniaxial anisotropy constant is set to $k=0.1$ for the rest of this chapter. We introduce a weak DMI to the system in order to break the invariance with respect to the rota- 


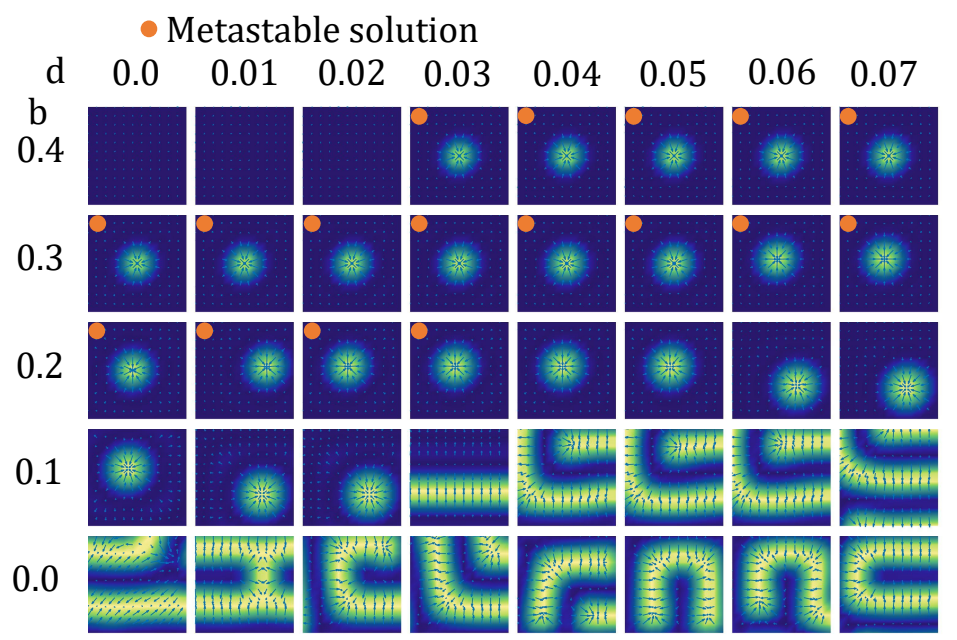

(a)

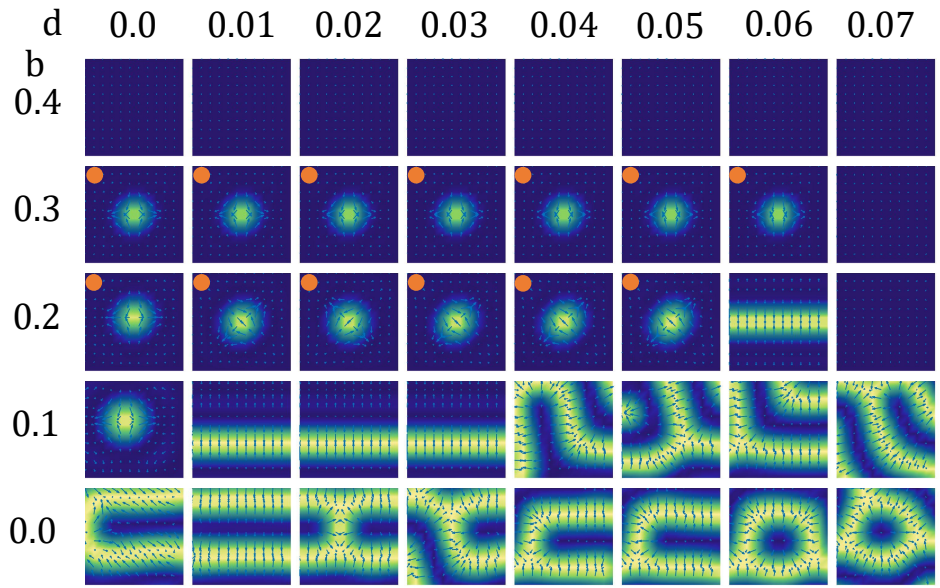

(b)

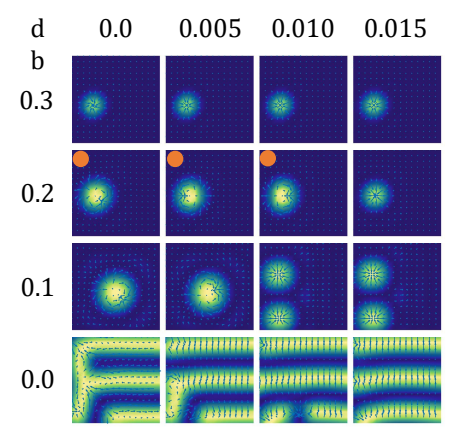

(c)

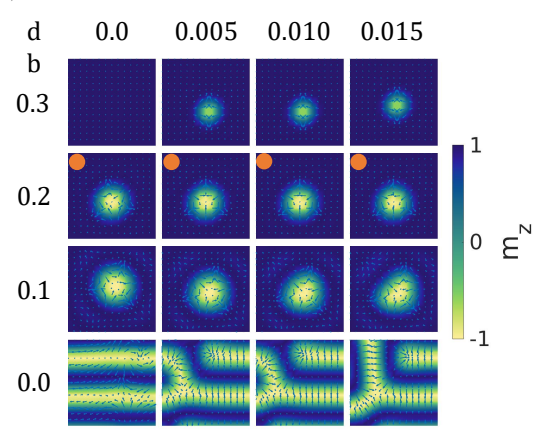

(d)

Figure 6.1: For $k=0.1$ and different values of the reduced DMI constant $d$ and the reduced applied field $b$, existence of (a) an isolated skyrmion solution, (b) an isolated antiskyrmion solution, (c) an isolated second-order skyrmion solution, (d) an isolated second-order antiskyrmion solution. Only metastable solutions of interest corresponding to excitations of the ferromagnetic ground state are marked with an orange dot. The spin maps are zoomed in to show the topological defects. 
tion of helicity, as well as the degeneracy between skyrmions and antiskyrmions. We look for a set of parameters that allow metastable skyrmion and antiskyrmion solutions to co-exist. To do so, we initialize the system close to a(n) (anti)skyrmion state and run the iterative energy minimization procedure described in Section 3.5.1. If a(n) (anti)skyrmion solution exists, it is relaxed. We vary the perpendicular destabilizing applied field $b$ in $[0,0.4]$, and the DMI coupling constant $d$ in $[0,0.07]$. We obtain the diagrams of Fig. 6.1 for skyrmions (Fig. 6.1a) and antiskyrmions (Fig. 6.1b).

At low field or high DMI, a spiral state is relaxed. Above a critical applied field, the spin-spiral state becomes the fully polarized ferromagnetic state via a second-order phase transition [25]. Within the FM phase, metastable skyrmion solutions can be stabilized. In the presence of a perpendicular easy-axis anisotropy, the skyrmion lattice becomes thermodynamically stable at intermediate applied field [25]. In this region, skyrmions are more favourable than the FM state. They are metastable in the sense that they are local energy solutions, but not the ground state. At finite temperature, the isolated skyrmion state is rapidly destroyed by the nucleation of many topological defects in its vicinity. By contrast, the metastable solutions we are interested in are isolated skyrmionic defects as excitations of the ferromagnetic ground state, which we mark as orange dots in Fig. 6.1. Since the symmetry of the DMI favours Néel skyrmions, degeneracies are lifted, and antiskyrmions become progressively less stable than their skyrmion counterparts as the DMI increases. They also relax diagonally on the square lattice, such that their Bloch-type axes, which we showed in Fig. 2.6, are along the first nearest-neighbour axes, which are the only ones that are coupled by the DMI. This is due to the fact that one of the Néel-type axes of the antiskyrmion exhibits the opposite chirality to the one favoured by the DMI, as was illustrated in Fig. 2.6 and discussed in Sec. 2.3. This introduces frustration in the orientation of the antiskyrmion solutions, which is not present in first-order skyrmion solutions with cubic symmetry.

Lastly, Figs. 6.1c and 6.1d show the range of existence of second-order skyrmion and antiskyrmion solutions (see Figs. $2.9 \mathrm{~d}$ and $2.9 \mathrm{e}$ ), respectively. They exist as metastable solutions at low $d$ in the skyrmion lattice phase, where they are more favourable than the FM state. By increasing the DMI at constant applied field, a bound skyrmion pair is relaxed instead of the second-order skyrmion, but the second-order antiskyrmion solution persists. Metastable solutions are found within a small window of the ferromagnetic phase at sufficiently low field and DMI. We find second-order skyrmions and antiskyrmions to be quasi-degenerate in energy whenever they both exist, with small differences most likely caused by the underlying lattice. However, the range of existence of the antiskyrmion appears to be wider. 


\subsection{Paths to annihilation}

Transition mechanisms correspond to minimum energy paths on the $2 \mathrm{~N}$-dimensional energy surface. Once more, we compute them via CI-GNEB [59] calculations with a climbing image scheme [58]to precisely identify the first-order saddle point on the path. In the GNEB method, successive states of the system along the reaction coordinate are referred to as images. In what follows, we present the annihilation mechanisms of a skyrmion (Fig. 6.2), an antiskyrmion (Fig. 6.4), and a second-order skyrmion (Fig. 6.6). For each mechanism, we plot the spin maps at selected images and the corresponding topological charge density, $\rho_{s}$. Note that the topological charge density was obtained by interpolating the skyrmion profile and using the continuous definition of Eq. (2.34). However, the discrete form of $\rho_{s}$, which we gave in Eq. (2.39), is the more correct definition that should be used on the atomistic spin lattice. The total energy along the path is plotted in Figs. 6.3, 6.5, and 6.7 for the skyrmion, the antiskyrmion, and the second-order skyrmion, respectively, in units of the isotropic exchange coupling constant between first neighbours, $J_{1}$, as well as the total topological charge, $N_{s}$, at each image, which was calculated according to the discrete definition in Eq. (2.38).

\subsubsection{Skyrmion}

The first set of parameters we examine is $(b, d)=(0.2,0.03)$. It is a region of parameter space where both metastable skyrmion and antiskyrmion solutions exist. In this context, we observe an annihilation mechanism which is different from the usually reported isotropic type of collapse. We show spin maps at selected images along the path in Fig. 6.2a, and the corresponding energy profile is shown in red in Fig. 6.3a, where the evolution of the topological charge is shown in Fig. 6.3b. The mechanism is as follows. One half of the skyrmion unfolds, and a half-antiskyrmion, or antimeron, is nucleated in its place (ims. [1-6]). The injection of the opposite topological charge in the system constitutes the first-order saddle point on the transition path (im. 6 in Fig. 6.2a and inset on the right in Fig. 6.3a), and, as shown in Fig. 6.3b, also coincides with the loss of the topological charge. This state possesses four different realizations, equivalent to rigid $\pi / 2$-rotations of the whole system. The remaining meron and antimeron then naturally annihilate (ims. [7-10]). We found that the isotropic type of collapse also exists in this system, but it involves a higher order saddle point.

The second set of parameters is $(b, d)=(0.3,0.07)$. In the space of parameters, it is situated just above the limit at which antiskyrmion solutions no longer exist. We show spin maps at selected images along the path in Fig. 6.2b, and the corresponding energy profile is shown in pale blue in Fig. $6.3 \mathrm{a}$, where the evolution of the topological charge 


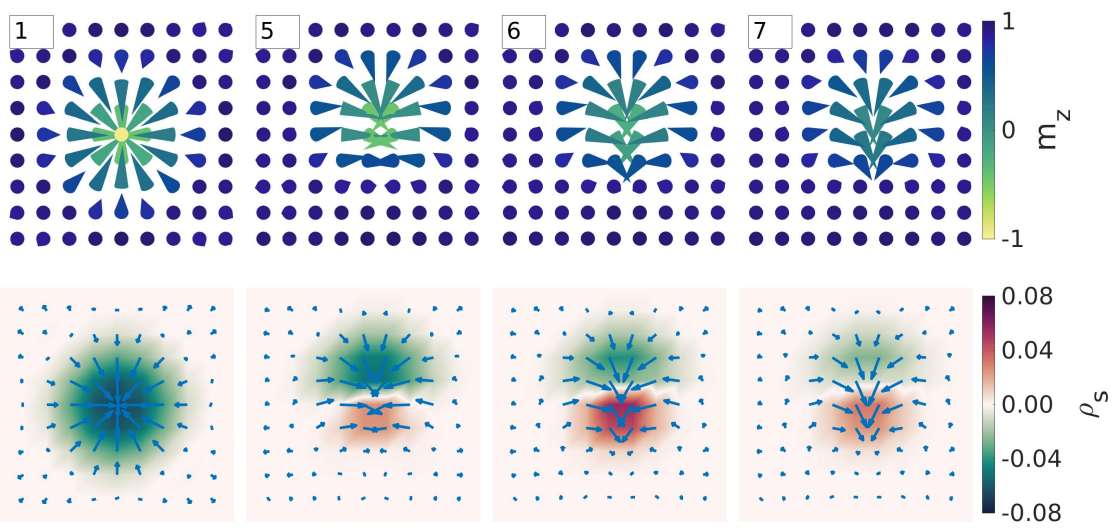

(a)

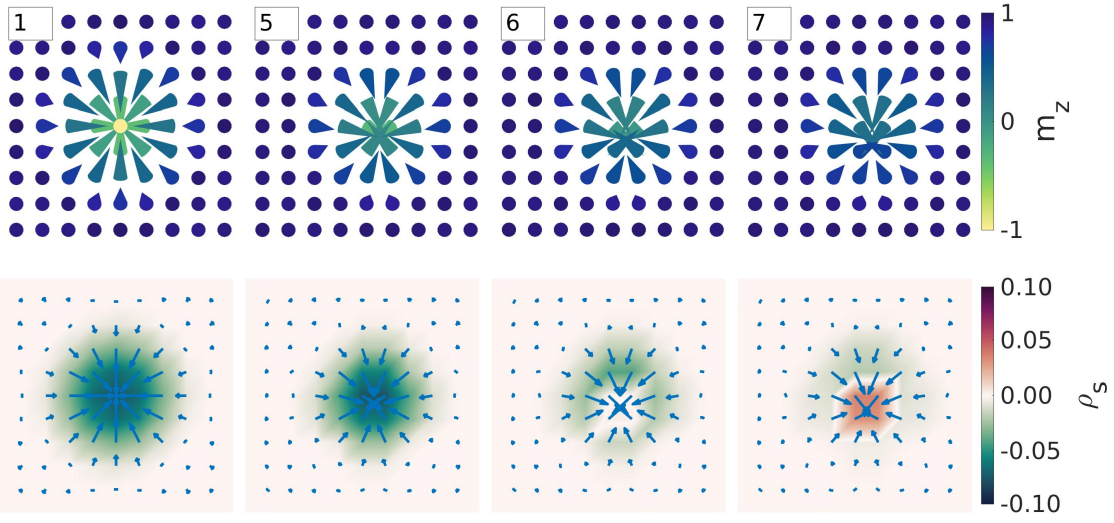

(b)

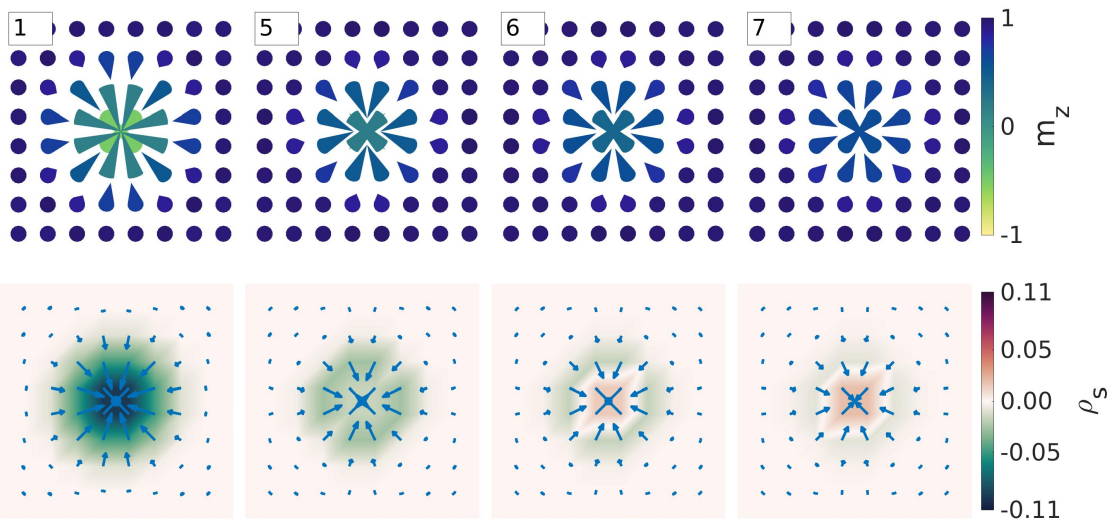

(c)

Figure 6.2: Spin maps (zoomed) and corresponding topological charge density along the transition path for a skyrmion annihilation. The parameters are (a) $(b, d)=$ $(0.2,0.03)$ (metastable antiskyrmion solutions exist), (b) $(b, d)=(0.3,0.07)$ (close to the existence of antiskyrmion solutions), (c) $(b, d)=(0.7,0.2)$ (antiskyrmions solutions do not exist). The image index is given in the top left-hand corner. When antiskyrmion solutions exist, the skyrmion annihilates via the nucleation of a half-antiskyrmion, or antimeron. The meron-antimeron state constitues the saddle point (im. 6). When antiskyrmion solutions do not exist, the isotropic collapse is recovered. 


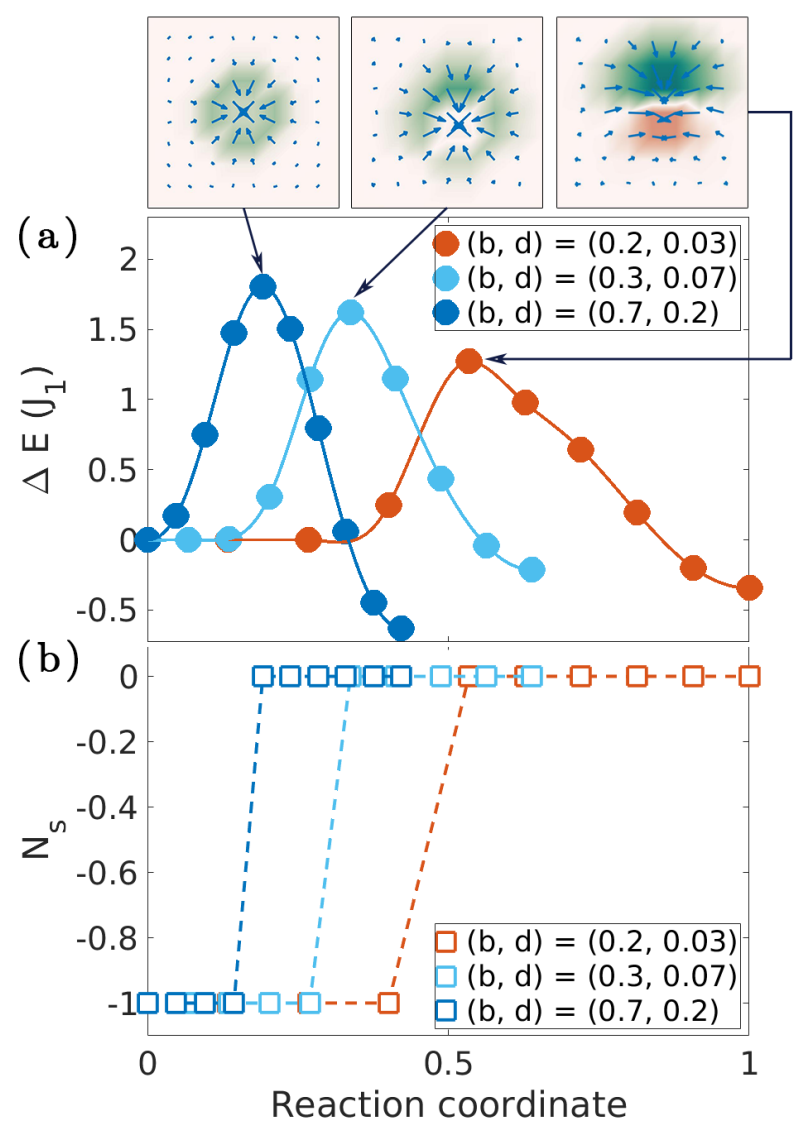

Figure 6.3: For the skyrmion annihilation at different values of the reduced applied field $b$ and reduced DMI constant $d$, we show the evolution along the transition path of (a) the internal energy barrier in units of $J_{1}$, (b) the topological charge. The reaction coordinate is normalized by the largest path length. The insets show the spin configuration and the topological charge density at the saddle point. In all three cases, the SP has a vanishing topological charge.

is shown in Fig. 6.3 b. In this case, the skyrmion undergoes a regular collapse involving a first-order saddle point (im. 6 in Fig. 6.2b and inset in the centre in Fig. 6.3a), but the core at the SP displays a weak asymmetry in a way that is reminiscent of the mechanism in Fig. 6.2a. For this reason, the saddle point is different from the saddle point configurations $S_{1}$ and $S_{2}$ that we reported in Chapter 4 in the case of the isotropic collapse of a chiral skyrmion. Since we are close to the region of antiskyrmions' metastability, this mechanism can be considered as intermediate between antimeron nucleation and isotropic collapse.

Finally, we select a region of parameter space with high DMI, far from the metastability region of antiskyrmions: $(b, d)=(0.7,0.2)$. We show spin maps at selected images along the path in Fig. 6.2c, and the corresponding energy profile is shown in dark blue in Fig. 6.3a, where the evolution of the topological charge is shown in Fig. 6.3b. As expected, we obtain a perfectly isotropic collapse similar to the ones reported in Chapter 
4, and without the injection of an opposite topological charge. In all three cases, the topological charge seems to fall to zero at the saddle point, although a higher resolution in the SP region could potentially reveal a state with a half-integer charge preceeding the SP.

Similar findings were reported very recently in Ref. [118]. In this work, the authors observed 5-nm skyrmions at zero magnetic field in the virgin FM phase of Rh/Co on $\operatorname{Ir}(111)$ via spin polarized STM. DFT calculations revealed a strong exchange frustration in this system. Subsequent atomistic GNEB simulations with a Hamiltonian parametrized by DFT showed that at zero field, the preferred collapse mechanism is similar to the one we reported here at low field and DMI in Fig. 6.2a, where the barrier results mainly from the strong isotropic exchange coupling between first nearestneighbours, $J_{1}$, more than from the DMI. At a higher applied field of $1 \mathrm{~T}$, the isotropic collapse was recovered, in agreement with the mechanisms that we presented in Figs. $6.2 \mathrm{~b}$ and $6.2 \mathrm{c}$ at higher field and DMI. In that case, isotropic collapse was preferred as it lowers the anisotropy and the Zeeman energy contributions. The existence of antiskyrmions in the system was not reported.

\subsubsection{Antiskyrmion}

We consider again the first set of parameters, $(b, d)=(0.2,0.03)$, which allows metastable antiskyrmions to coexist with skyrmions. We look for paths to annihilation of an antiskyrmion, and we relax two different mechanisms passing through a firstorder saddle point, for which we show spin maps at selected images in Figs. 6.4a and 6.4b, where the corresponding energy profiles are shown in Figs. 6.5a and 6.5b. In both cases, the annihilation process begins with the rotation of the helicity of the antiskyrmion, such that its Néel-type axes are aligned along the first-neighbour axes (ims. [1-7]). This configuration constitutes an energy maximum as it is unfavoured by the DMI, and the antiskyrmion can reach this state either as a full antiskyrmion with full integer topological charge ( $\mathrm{SP}_{2}$ at im. 7 in Figs. $6.4 \mathrm{~b}$ and $\left.6.5 \mathrm{~b}\right)$, or the spins may begin to unwind, which leads to a half-integer topological charge ( $\mathrm{SP}_{1}$ at im. 7 in Figs. 6.4a and 6.5a). Past the saddle point, similarly to the annihilation mechanism of its skyrmion counterpart, the topological charge drops to zero as a meron is nucleated (im. 8) and annihilates with the remaining antimeron (ims. [9-10]). $\mathrm{SP}_{1}$ exists in four possible realizations $\left(\pi / 2\right.$-rotations), while $\mathrm{SP}_{2}$ exists in two ( $\pi$-rotation). Therefore, even though the barrier associated with $\mathrm{SP}_{2}$ appears very small, it is necessary to compute an attempt frequency before drawing any conclusion on whether or not the mechanism involving $\mathrm{SP}_{1}$ should contribute to the total rate. It is interesting to note that the fact that the antiskyrmion displays frustration in its helicity suffices to distinguish between 


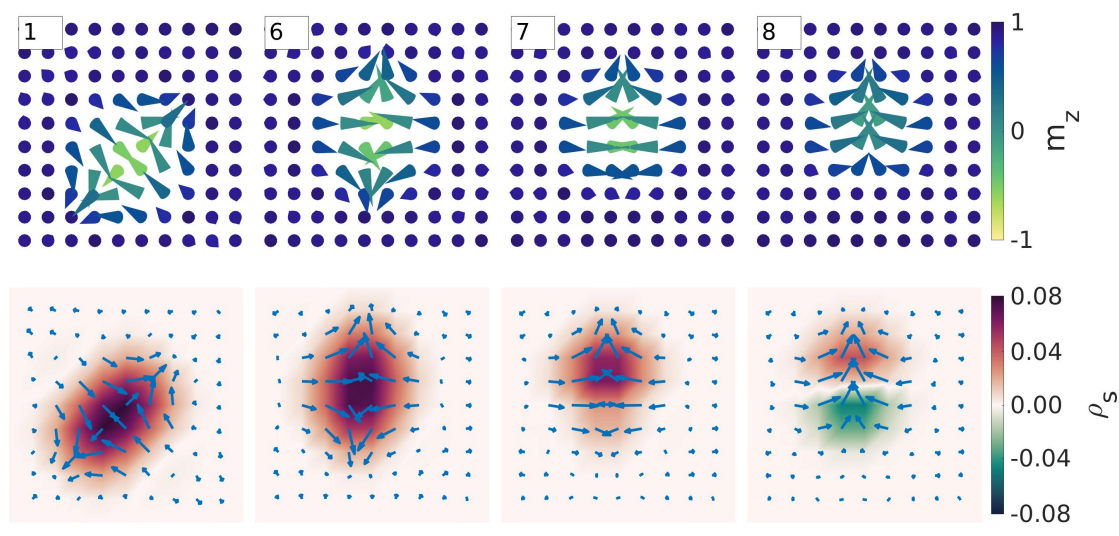

(a)

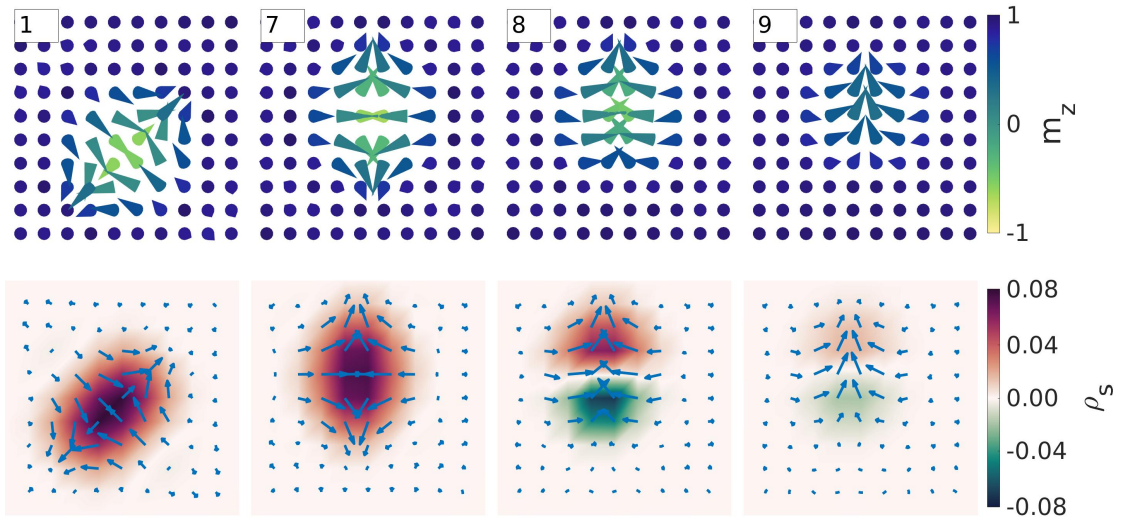

(b)

Figure 6.4: Spin maps (zoomed) and corresponding topological charge density along the transition path for an antiskyrmion annihilation with $(b, d)=(0.2,0.03)$ where (a) shows the path over $\mathrm{SP}_{1}$ and (b) shows the path over $\mathrm{SP}_{2}$. The image index is given in the top left-hand corner. The antiskyrmion annihilates via the nucleation of a half skyrmion, or meron. The saddle point is im. 7 in both cases and preceeds the meron nucleation, which occurs at im. 8. 


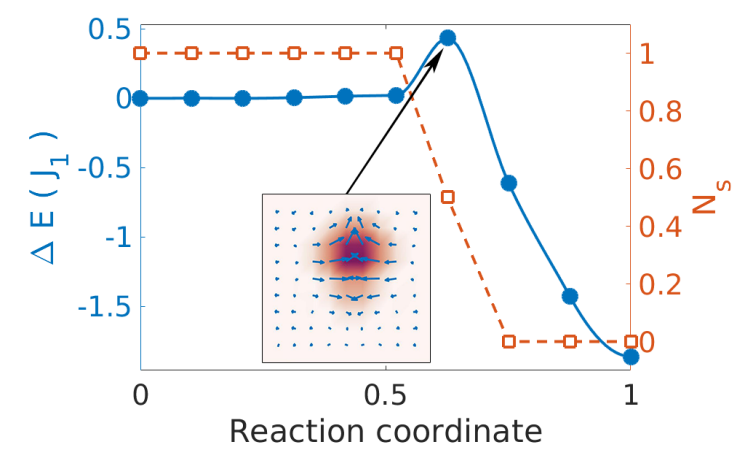

(a)

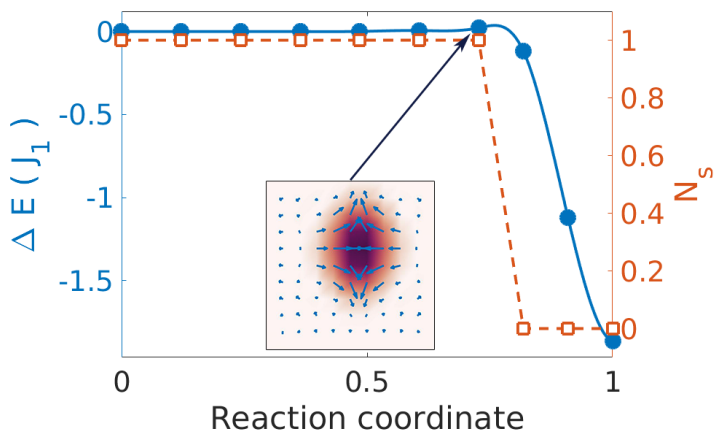

(b)

Figure 6.5: Internal energy barrier in units of $J_{1}$ (filled blue dots) and topological charge $N_{s}$ (unfilled red squares) along the transition path for an antiskyrmion annihilation with $(b, d)=(0.2,0.03)$ for $(\mathrm{a})$ the path over $\mathrm{SP}_{1}$, (b) the path over $\mathrm{SP}_{2}$. The inset shows the spin configuration and the topological charge density at the saddle point. $\mathrm{SP}_{1}$ carries a $1 / 2$ topological charge, while $\mathrm{SP}_{2}$ carries a full integer charge.

a metastable solution and a saddle point. In other words, the saddle point $\mathrm{SP}_{2}$ corresponds to the same configuration as the metastable antiskyrmion state, but with a $\pi / 4$-rotation of helicity.

\subsubsection{Second-order skyrmion}

We select parameters that allow both the first-and second-order skyrmions to be metastable: $(b, d)=(0.14,0.005)$. We initially set the final state of the GNEB calculation to a Néel skyrmion, and we obtain the mechanism in Fig. 6.6a, with the corresponding energy and topological charge profiles shown in Fig. 6.7a. The second-order skyrmion first undergoes a rotation of helicity (ims. [1-3]), and reaches the saddle point (im. 3). The nucleation of an antimeron along the Néel axis follows at image 4 , at which point the energy drops, and the topological charge goes from $N_{s}=-2$ to $N_{s}=-1+\frac{1}{2}-\frac{1}{2}=$ -1 . The antimeron subsequently annihilates with part of the second-order skyrmion, leaving a first-order Néel skyrmion in its place (ims. [4-8]).

Another route to annihilation for the second-order skyrmion corresponds to its division into a bound skyrmion pair, as shown in Fig. 6.6b. The corresponding energy and topological charge profiles are shown in Fig. 6.7b. The bound skyrmion pair is a metastable solution, since the interaction potential of skyrmions in frustrated magnets is non-monotonic as a function of distance, and is found in turn positive (repulsive) and negative (binding) [22, 25]. This behaviour is not present in chiral magnets, in which the interaction between skyrmions is always repulsive. This mechanism does not involve any significant change in the topological charge of the total system, but instead results in a redistribution of the topological charge density. The saddle point in this 


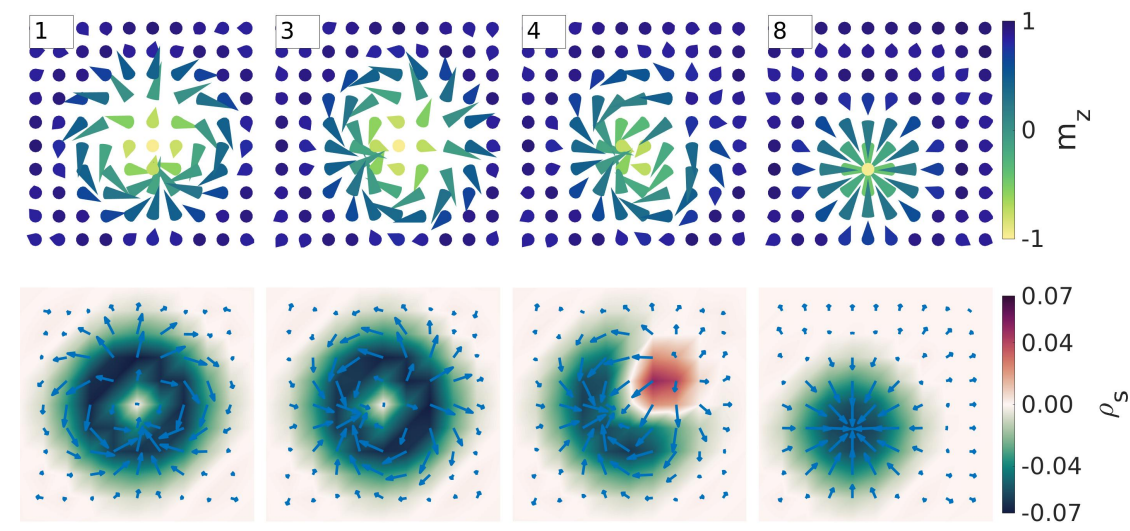

(a)

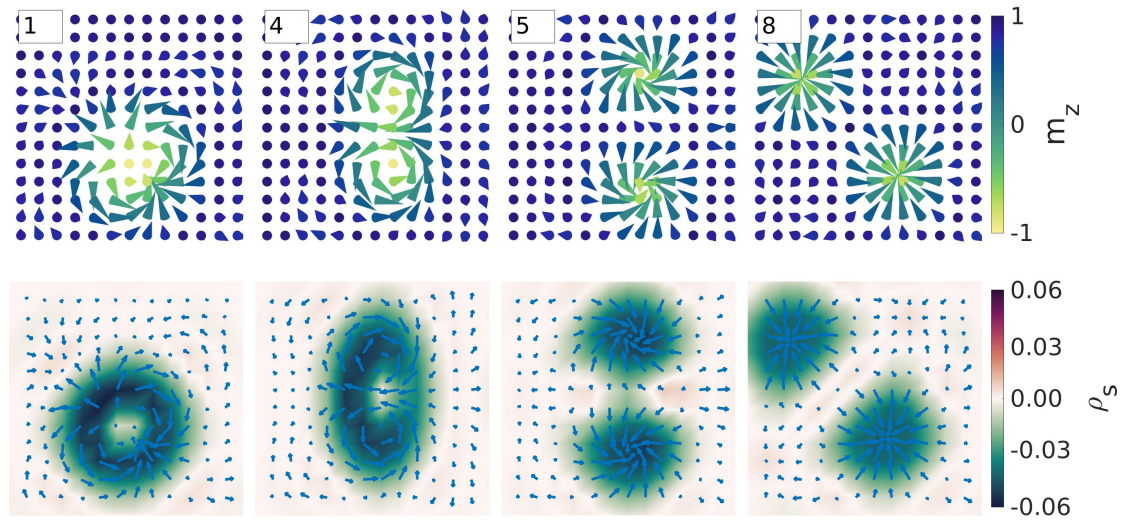

(b)

Figure 6.6: Spin maps (zoomed) and corresponding topological charge density along the transition path for (a) the decay of a second-order skyrmion into a first-order skyrmion via antimeron nucleation, with $(b, d)=(0.14,0.005)$, (b) the division of a secondorder skyrmion into a bound skyrmion pair, with $(b, d)=(0.1,0.005)$. The image index is given in the top left-hand corner. In (a), the SP is im. 3 and preceeds the antimeron nucleation. In (b), the SP is im. 4 and preceeds the separation of the two skyrmions.

case is the state preceeding the separation of the two skyrmions (im. 4). Both of the above mechanisms possess four realizations, corresponding to $\pi / 4$ rigid rotations of the system. As for the fate of the skyrmion pair, the binding potential is quite shallow compared to the activation barrier for annihilation (from the results of Ref. [25], we can estimate the unbinding barrier to be $\left.\sim 10^{-4} J_{1}\right)$. Therefore, the skyrmions will, in most cases, separate before annihilating individually.

Similar processes were reported in Ref. [27] from dynamics simulations, in which the second-order skyrmion division was triggered by both a driving current at zero temperature, and thermal fluctuations at finite temperature. The finite temperature simulations also showed the decay of the second-order skyrmion into a first-order skyrmion. 


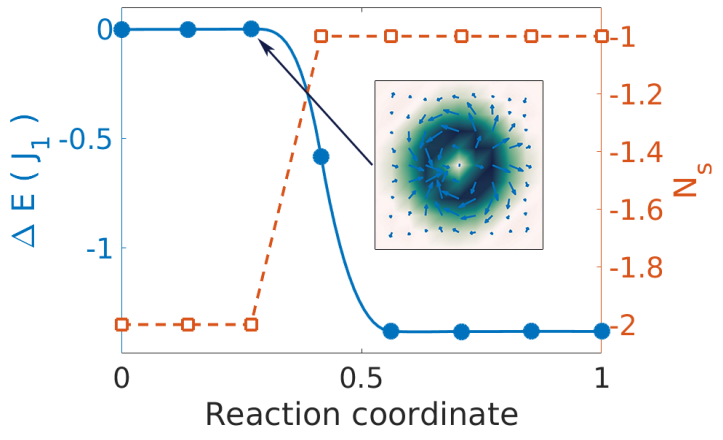

(a)

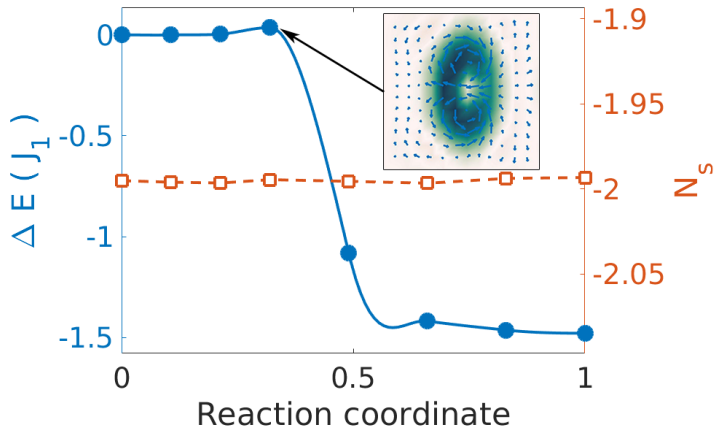

(b)

Figure 6.7: Internal energy barrier in units of $J_{1}$ (filled blue dots) and topological charge $N_{s}$ (unfilled red squares) along the transition path for (a) the decay of a second-order skyrmion into a first-order skyrmion via antimeron nucleation, with $(b, d)=(0.14,0.005)$, (b) the division of a second-order skyrmion into a bound skyrmion pair, with $(b, d)=(0.1,0.005)$. The inset shows the spin configuration and the topological charge density at the saddle point.

\subsection{4 (Anti)meron nucleation and evolution of the topological charge}

In order to visualize the evolution of the topological charge, we show the "ball in a net" representations of some annihilation mechanisms presented above in Fig. 6.8. As previously described in Sec. 2.3.2, vertices correspond to the tip of the magnetic vectors with their origin in the centre of the sphere, and the edges represent the exchange coupling between first neighbours. The ferromagnetic background (spins along $+z$ ) is found at the north pole of the sphere, while the core of a skyrmion typically points along the south pole (along $-z$ ). The view is set just below the south pole, looking axially towards the $+z$ direction, so that the core of the skyrmion is found at the centre of the view.

We show a skyrmion annihilation via antimeron nucleation (Fig. 6.8a) and isotropic collapse (Fig. 6.2b), an antiskyrmion annihilation via meron nucleation (Fig. 6.4b, which is the path that traverses $\mathrm{SP}_{1}$ ), and the decay of a second-order skyrmion via antimeron nucleation (Fig. 6.8d). Annihilations that occur via the injection of the opposite topological charge (Figs. 6.8a and 6.8c) involve a rearrangement of the spin-mesh in a way that allows the entire net to be removed along a single direction. By contrast, an isotropic collapse consists in progressively spreading the mesh open by an equal amount along all directions (Fig. 6.8b). Lastly, a second-order skyrmion corresponds to a spin configuration in which the magnetization wraps the unit sphere twice, therefore, in Fig. 6.8d, it looks like the ball is inside a double net. The decay into a first-order skyrmion via the injection of an antimeron consists in the removal of one of the nets, again along a given direction, so that only a single net remains. 


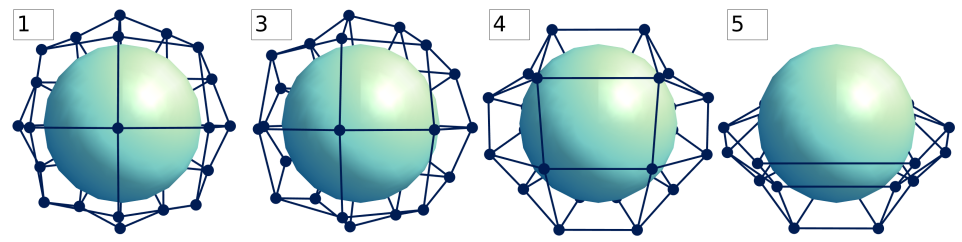

(a)
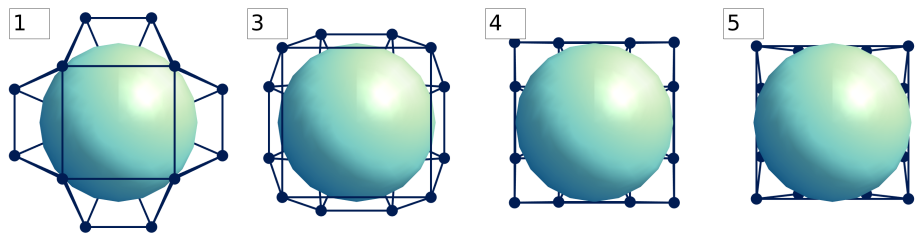

(b)
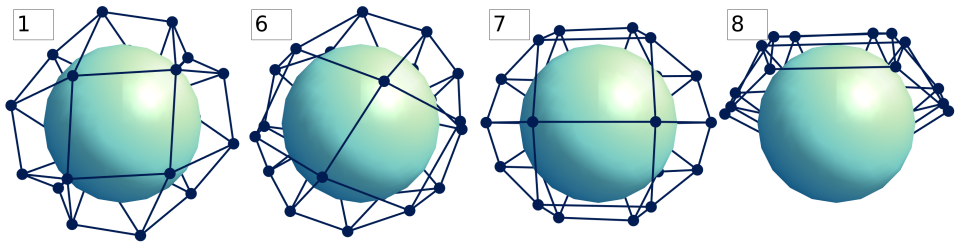

(c)
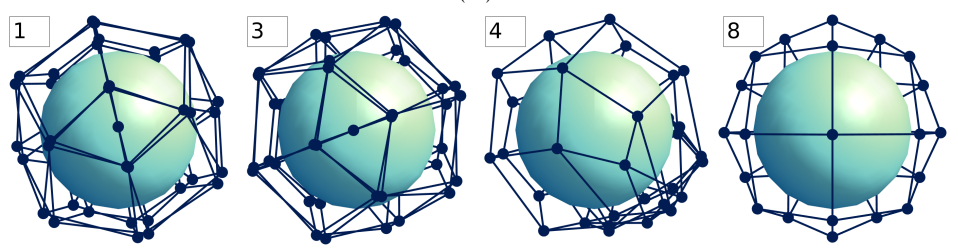

(d)

Figure 6.8: "Ball in a net" representation of the spin configuration at selected images along the path to annihilation. The image index is given in the top left-hand corner. We show (a) a skyrmion annihilation via antimeron nucleation (Fig. 6.2a), (b) a skyrmion annihilation via isotropic collapse (Fig. 6.2c), (c) an antiskyrmion annihilation via meron nucleation (Fig. 6.4a), (d) a second-order skyrmion decay into a first-order skyrmion (Fig. 6.6a).

\subsection{Langevin simulations}

In order to validate the previous paths calculated with GNEB, we perform direct Langevin simulations at low temperature. Details on the method are given in Sec. 5.2 . The values of the parameters are the same as in Chapters 4 and $5 J_{1}=1.6 \times 10^{-20} \mathrm{~J}$, $\mathscr{M}_{s}=1.1 \times 10^{6} \mathrm{~A} . \mathrm{m}^{-1}, \alpha=0.5$, and $\gamma_{e}=1.76 \times 10^{11} \mathrm{rad} . \mathrm{s}^{-1} \cdot \mathrm{T}^{-1}$. We record annihilation mechanisms for which we show snapshots of the spin configuration in Fig. 6.10.

For the antiskyrmion, at $T=80 \mathrm{~K}$ and $(b, d)=(0.2,0.03)$, which we show in Fig. 6.10 a, we observe the meron-nucleation type of annihilation that we reported in Fig. 6.4. Since we have short lifetimes within the nanosecond time scale, we can estimate the antiskyrmion's stability directly from Langevin simulations. We use the topological 


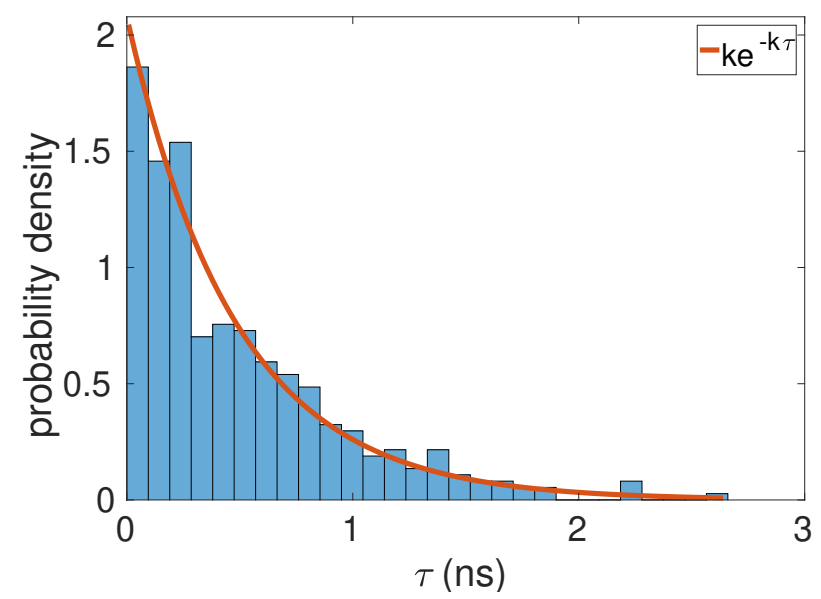

Figure 6.9: Probability density distribution of the lifetimes of the antiskyrmion computed over 390 direct Langevin simulations at $T=80 \mathrm{~K}$. The red curve is the theoretical PDF obtained with $k=k_{\text {Langer }}$ given in Eq. (6.1).

charge of the system to track the collapse of the antiskyrmion, that is, a collapse is recorded when $N_{s}=0$. We record 390 collapses and obtain an average lifetime $\tau_{\text {Lgv }}(80$ $\mathrm{K})=0.49 \mathrm{~ns}$, with a standard deviation of the order of the mean lifetime, $\sigma=0.46$ $\mathrm{ns}$. The fact that the standard deviation is approximately the same as the mean value is coherent with the properties of an exponentially distributed variable such as $\tau$. Fig. 6.9 shows the probability density distribution of the computed lifetimes, which matches the probability density function (PDF) $f(k, \tau)=k e^{-k \tau}$ plotted in red, where we have used $k=k_{\text {Langer }}$ given in Eq. (6.1).

As for the second-order skyrmion and antiskyrmion, as they overall exhibit lower activation energies, we perform simulations at $T=50 \mathrm{~K}$, and $(b, d)=(0.14,0.005)$. The division of the second-order skyrmion into a bound skyrmion pair seems to occur along at least two different paths that we show in Figs. 6.10b and 6.10 c, in which the latter corresponds to the MEP in Fig. 6.6b. In Fig. 6.10b, the division occurs through excitation of one half of the second-order skyrmion, which eventually leads to the separation of the two halves. On the other hand, in Fig. 6.10k, we witness a more symmetric kind of division. For the same set of parameters, we also observe the decay into a single skyrmion shown in Fig. 6.10d, which corresponds to the path in Fig. 6.6a. Finally, in Fig. 6.10e, we show the division of a second-order antiskyrmion into a pair of firstorder antiskyrmions. Since the pairwise interaction potential between antiskyrmions depends on their relative helicities in addition to their distance [25], this mechanism is difficult to relax with GNEB due to the overall complexity of the energy landscape, so we only observed this particular mechanism via Langevin simulations. 


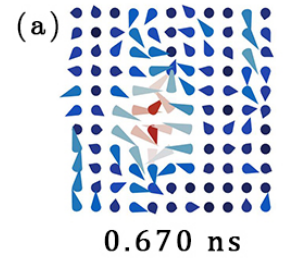

(b)

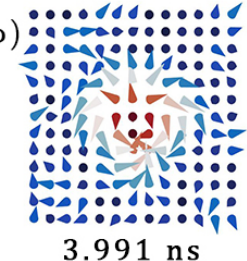

(c)

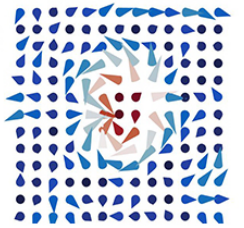

$1.612 \mathrm{~ns}$

(d)

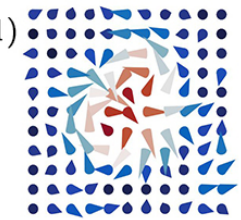

$0.999 \mathrm{~ns}$

(e)

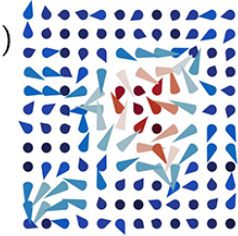

$2.967 \mathrm{~ns}$

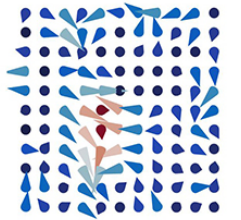

$0.672 \mathrm{~ns}$

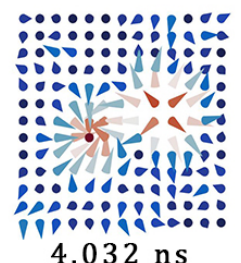

$4.032 \mathrm{~ns}$

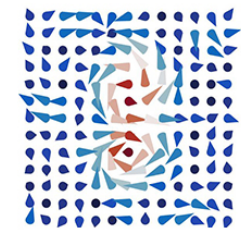

$1.623 \mathrm{~ns}$

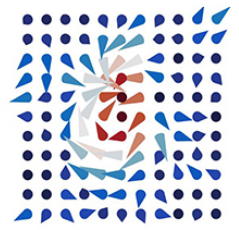

$1.003 \mathrm{~ns}$

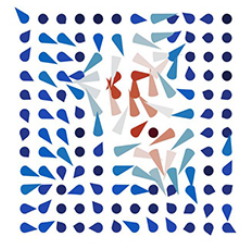

$2.981 \mathrm{~ns}$

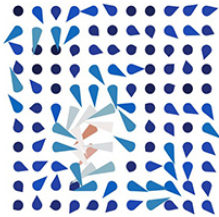

$0.675 \mathrm{~ns}$

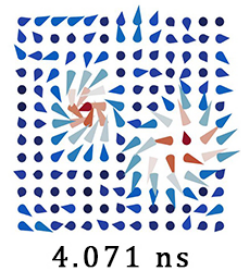

$4.071 \mathrm{~ns}$

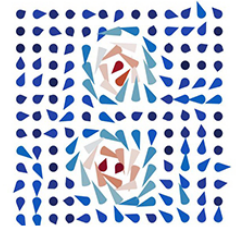

$1.638 \mathrm{~ns}$

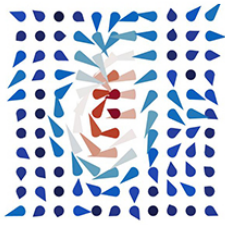

$1.004 \mathrm{~ns}$

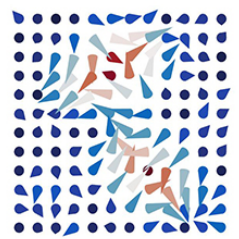

$3.021 \mathrm{~ns}$

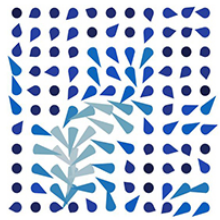

$0.677 \mathrm{~ns}$

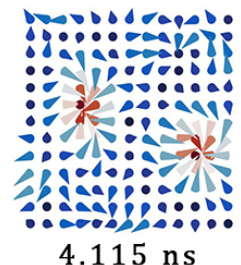

$4.115 \mathrm{~ns}$

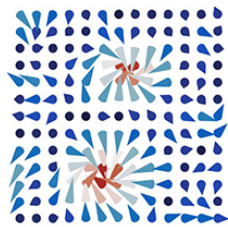

$1.644 \mathrm{~ns}$

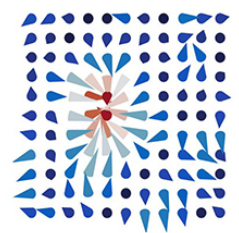

$1.011 \mathrm{~ns}$

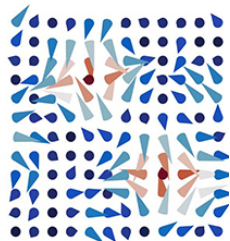

$3.049 \mathrm{~ns}$

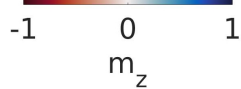

Figure 6.10: Langevin simulation snapshots (zoomed) of (a) an antiskyrmion annihilation, $(b, c)$ the division of a second-order skyrmion into a bound skyrmion pair, $(d)$ the decay of a second-order skyrmion into a first-order skyrmion, (e) the division of a second-order antiskyrmion into a bound antiskyrmion pair. The antiskyrmion annihilation is shown at $T=80 \mathrm{~K},(b, d)=(0.2,0.03)$, while the other simulations are carried out at $T=50 \mathrm{~K},(b, d)=(0.14,0.005)$.

\subsection{Thermal stability}

We use Langer's theory to compute a complete rate for each of the mechanism presented above. The results are gathered in Table 6.1. To account for all equivalent realizations of a given mechanism (rigid rotations of the spin configurations at the saddle point), the prefactor $f_{0}$ is multiplied by a factor two or four when it is appropriate, 


\begin{tabular}{|c|c|c|c|c|}
\hline Mechanism & $b\left(J_{1}\right)$ & $d\left(J_{1}\right)$ & $\Delta E\left(J_{1}\right)$ & $f_{0}(\mathrm{GHz})$ \\
\hline & & Skyrmion & & \\
\hline Antimeron nucleation & 0.2 & 0.03 & 1.27 & $109.9 \times 4$ \\
\hline Isotropic collapse & 0.3 & 0.07 & 1.62 & 3639.5 \\
\hline \multirow[t]{2}{*}{ Isotropic collapse } & 0.7 & 0.2 & 1.80 & 1247.5 \\
\hline & & Antiskyrmion & & \\
\hline Meron nucleation $\mathrm{SP}_{1}$ & 0.2 & 0.03 & 0.43 & $10.6 \times 4$ \\
\hline \multirow[t]{2}{*}{ Meron nucleation $\mathrm{SP}_{2}$} & 0.2 & 0.03 & 0.022 & $1.4 \times 2$ \\
\hline & & $\begin{array}{c}\text { 2nd. order } \\
\text { sk. }\end{array}$ & & \\
\hline Antimeron nucleation & 0.14 & 0.005 & 0.0011 & - \\
\hline Division & 0.14 & 0.005 & 0.062 & - \\
\hline
\end{tabular}

Table 6.1: Internal energy barrier, $\Delta E$, and rate prefactor, $f_{0}$, for the annihilation of a skyrmion, an antiskyrmion, and a second-order skyrmion with different values of the reduced applied field $b$ and reduced DMI $d$.

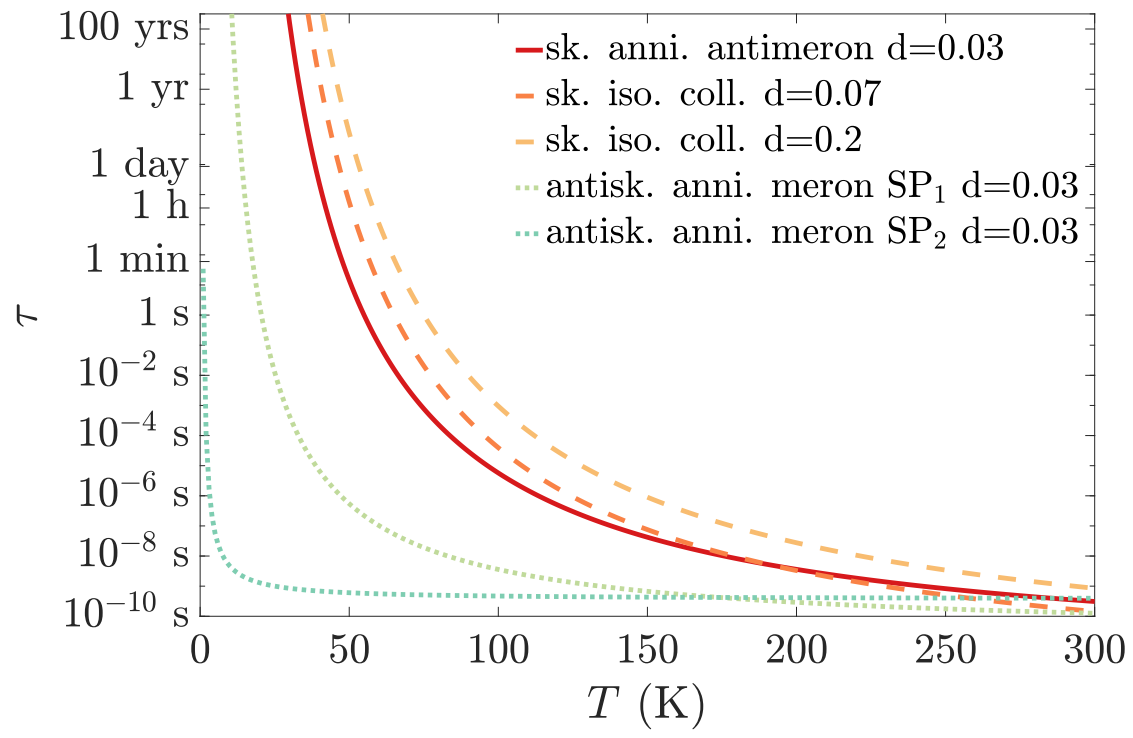

Figure 6.11: Average lifetime of a skyrmion and an antiskyrmion under frustrated exchange as a function of temperature with respect to all considered mechanisms calculated via Langer's theory as given in Eq. (3.31). $k=0.1$ in all cases. The value of $b$, the energy barriers and the value of the prefactor are given in Table 6.1. 
in accordance with Sec. 6.3. In the case of the decay of the second-order skyrmion into a first-order skyrmion, we find a Goldstone mode at both the metastable and transition states, which do not seem to clearly correspond to a translational or rotational invariance, hence we cannot give a definite value for the attempt frequency in this case. Based on these results, we can estimate the lifetime of the antiskyrmion calculated with Langer's theory, and compare it with the average lifetime previously obtained from direct Langevin simulations. Assuming independent processes, we have

$$
\begin{aligned}
k_{\text {Langer }}(80 \mathrm{~K}) & =f_{01} e^{-\beta \Delta E_{1}}+f_{02} e^{-\beta \Delta E_{2},} \\
& =\tau_{\text {Langer }}^{-1}(80 \mathrm{~K}), \\
& =2.08 \mathrm{GHz},
\end{aligned}
$$

and $\tau_{\text {Langer }}(80 \mathrm{~K})=0.48 \mathrm{~ns}$. Although we are not completely within the scope of Langer's theory $\left(\beta \Delta E_{1}=0.3\right.$, as opposed to the recommended $\beta \Delta E \geq 5$ [47]), this shows an excellent agreement with the result from direct Langevin, $\tau_{\mathrm{Lgv}}(80 \mathrm{~K})=0.49 \mathrm{~ns}$ (see also the agreement of the probability density in Fig. 6.9).

In Fig. 6.11, we show the average lifetimes of the skyrmion and the antiskyrmion as a function of temperature with respect to the different mechanisms. Overall, the structures are much less stable than the chiral solutions in Chapter 4 . The average lifetimes at room temperature are typically less than a nanosecond. This is not surprising, because the barriers are small compared to the exchange strength $\left(\Delta E_{\mathrm{sk}}<2 J_{1}\right.$ for the skyrmion and $\Delta E_{\text {antisk }}<1 J_{1}$ for the antiskyrmion), and the attempt frequencies are of the order of $1-1000 \mathrm{GHz}$. The more stable skyrmion is the skyrmion at higher DMI $(d=0.2)$ which annihilates via isotropic collapse. This is because it has the highest barrier, and second lowest attempt frequency. At $300 \mathrm{~K}$, its lifetime is $0.84 \mathrm{~ns}$. At low temperature, the least stable skyrmion is the one annihilating via antimeron nucleation at low DMI $(d=0.03)$, although, at room temperature, even though it has the lowest energy barrier, it is more stable than the solution at intermediate DMI $(d=0.07)$ which undergoes a collapse with a small asymmetry. This is because the attempt frequency associated with meron nucleation is one order of magnitude smaller than the ones associated with collapse mechanisms. The antiskyrmion also provides an interesting example where the attempt frequency plays an essential role. The energy barrier associated with $\mathrm{SP}_{2}$ is very small compared to the exchange strength $\left(0.022 \mathrm{~J}_{1}\right)$ and much smaller than the one associated with $\mathrm{SP}_{1}$, so one would typically assume that the mechanism involving $\mathrm{SP}_{2}$ should dominate the rate. However, the attempt frequency associated with $\mathrm{SP}_{2}$ is not only ten times lower than the one associated with $\mathrm{SP}_{1}$, but $\mathrm{SP}_{2}$ can be realized in two different ways, i.e., whenever the helicity of the antiskyrmion is 0 or $\pi$, while $\mathrm{SP}_{1}$ can be realized in four different ways, that is, the inset in Fig. $6.4 \mathrm{a}$ and 
three $\pi / 4$ rotations. It follows that the total attempt frequency associated with $\mathrm{SP}_{1}$ is 15 times larger than the one associated with $\mathrm{SP}_{2}$. Consequently, $\mathrm{SP}_{2}$ dominates the rate at very low temperature $(\mathrm{T} \ll 100 \mathrm{~K})$, but the lifetimes intersect around $175 \mathrm{~K}$. Beyond that limit, the mechanism involving $\mathrm{SP}_{1}$ is the most probable one. Even at $80 \mathrm{~K}$, the contribution from both saddle points is necessary for Langer's result to match the average lifetime and the probability density distribution of the lifetimes obtained from direct Langevin simulations (see Fig. 6.9).

\subsection{Discussion}

We have seen that, under frustrated exchange couplings, and in regions of parameter space for which both metastable skyrmion and antiskyrmion solutions can be realized, the most probable paths to annihilation appear to be the paths involving the injection of the opposite topological charge into the system, by means of the nucleation of merons and antimerons. The injection of the opposite charge usually happens shortly after the system has passed the saddle point configuration, or at the saddle point in the case of the skyrmion, and also coincides with the loss of one unit of topological charge. The state preceding the injection of the opposite charge may also possess a half-integer topological charge, as is the case of the antiskyrmion in the annihilation path involving the saddle point $\mathrm{SP}_{1}$ (im. 7 in Figs. 6.4a, 6.5a, and 6.8c).

Overall, the skyrmionic solutions in this system were found to be rather unstable, as they possess low internal energy barriers compared to the exchange strength, combined with many possible paths to annihilation with attempt frequencies in the range of several gigahertz to several hundreds of gigahertz. The existence of many possible paths is a direct consequence of the exchange frustration, which drastically complexifies the energy landscape and is responsible for the emergence of many (meta)stable and saddle point states, as well as many possible paths connecting them. At room temperature, the average lifetimes were calculated to be under a nanosecond. We conclude that this specific set of exchange parameters on the square lattice derived in Ref. [25] does not seem to yield isolated skyrmion solutions that could potentially be used in applications. In the present case, it seems that the skyrmion stabilized at higher DMI which undergoes isotropic collapse is a more stable solution than the low DMI solution whose annihilation occurs via antimeron nucleation. Additionally, the low DMI skyrmion might also undergo isotropic collapse. This mechanism is associated with a higher-order saddle point which we did not relax here, and it would contribute to the reduction of the skyrmion's lifetime. From there, one may conclude that the low DMI region of parameter space where metastable skyrmions are primarily stabilized 
by exchange frustration and antiskyrmions are also metastable solutions is not a region where isolated skyrmions will be the most stable, and so the existence of antiskyrmions should be an indicator of lower stability of the skyrmions. However, the attempt frequency of the meron nucleation is one order of magnitude lower than that of isotropic collapse processes. Therefore, as is often the case, things are not that simple, and the above conclusions only apply to the specific set of parameters we have studied in this chapter. In Ref. [2], we concluded that skyrmions undergo annihilation via antimeron injection in the region of parameter space where low DMI allows antiskyrmions to coexist with skyrmions as metastable solutions. With the selected set of parameters, we also had to increase the applied field with increasing DMI in order to be able to relax isolated (anti)skyrmion solutions (see the position of the orange dots in Fig. 6.1). At higher DMI and applied field, antiskyrmion solutions disappear and we recovered an isotropic type of collapse. In Ref. [118], small skyrmions were observed at zero field in $\mathrm{Rh} / \mathrm{Co} / \operatorname{Ir}(111)$ via SP-STM. A strong exchange frustration in this system was found from DFT calculations. GNEB simulations revealed that in $\mathrm{Rh} / \mathrm{Co} / \mathrm{Ir}(111)$, skyrmions at zero field undergo a similar annihilation to the one we have reported in this chapter, involving the injection of the opposite topological charge - which the authors referred to as "chimera" [26] collapse. At higher applied field, the isotropic collapse is favoured. This conclusion agrees partially with the results of this chapter. We did not find here a limit where, by increasing the field at constant DMI, the annihilation mechanism would change, however this still may be the case in a very small window of increasing applied field before skyrmions are no longer solutions, in which case it is possible that we missed it. Instead, we had to increase both the field and DMI to recover the isotropic collapse. The existence of antiskyrmions was not mentioned in Ref. [118]. We note that the system studied by the authors of Ref. [118] used a Co magnetic layer and sub-10-nm skyrmions were reported in the FM state at zero field. This configuration resembles the type of stabilization conditions of we have studied in Chapter 4 for DMI stabilization, and in the present chapter for the effect of exchange frustration. In all cases we used material parameters of $\mathrm{Pt} / \mathrm{Co} / \mathrm{AlO}_{x}$ for $J_{1}$ and the saturation magnetization. The energy barriers we have found here for the skyrmion are of a few hundreds of $\mathrm{meV}(127-180 \mathrm{meV})$, while in [118], they were calculated to be around 250-290 $\mathrm{meV}$, which is similar to the barriers we reported for chiral skyrmion collapse in Chapter 4 . This hints at a potential higher stability of the skyrmions in $\mathrm{Rh} / \mathrm{Co} / \operatorname{Ir}(111)$ than what we have reported in this chapter. However, a rate prefactor was not computed in Ref. [118].

The low stability of the studied solutions allowed for a direct comparison of the average lifetime of the antiskyrmion at $80 \mathrm{~K}$ computed from direct Langevin simulations with Langer's theory calculations, and we obtained a very good agreement. Neverthe- 
less, in this context, the direct use of reaction rate theory to estimate an overall lifetime for any given structure seems ill-advised and unreliable, as it is difficult to account for all possible mechanisms. As for the low activation energies and not particularly low attempt frequencies, we can once more relate them to the skyrmions being very small, and possessing only a few internal modes [1, 109]. Lastly, this chapter yet again contained some interesting examples where the attempt frequency plays an important role in the stability, especially in the higher temperature region. The most striking case is that of the antiskyrmion, which annihilates in this system via meron nucleation. One of its annihilation mechanisms involves a very small activation barrier of $0.022 J_{1}$ at saddle point $\mathrm{SP}_{2}$, yet, above a temperature of $175 \mathrm{~K}$, this mechanism yields a longer lifetime that the other mechanism over $\mathrm{SP}_{1}$, which corresponds to an activation energy of $0.43 J_{1}$. Even at $80 \mathrm{~K}$, we found that both mechanisms should be taken into account in Langer's theory calculations in order to match the results of direct Langevin simulations. 


\section{Chapter 7}

\section{Conclusion and outlook}

In this thesis, we have studied the stability of metastable magnetic skyrmions on the two-dimensional square lattice. This task was firstly carried out via a numerical implementation of Kramers' method for a multidimensional energy landscape set in the intermediate-to-high damping regime, i.e., Langer's statistical theory for the decay of metastable states. The paths of minimum energy that lead to the skyrmion annihilation were computed via the geodesic nudged elastic band method. Chiral magnetic skyrmions stabilized at zero magnetic field by the Dzyaloshinskii-Moriya interaction were found to annihilate via two distinct isotropic collapse mechanisms, respectively passing through a first-, and a third-order saddle point on the energy landscape. In both cases, the loss of the topological charge occurs past the saddle point. Alternatively, the skyrmions may diffuse to the edge of the system and escape through the boundary. The computation of a rate prefactor revealed that the thermally significant eigenmodes are the modes localized to the skyrmion, in contrast to the rest of the collective spin-wave modes, which extend to the entire lattice and contribute weakly. Important variations of the rate prefactor, also known as the attempt frequency, were found between different mechanisms on the one hand, and between different values of the destabilizing applied magnetic field on the other. These variations originate from strong entropic effects, which come from the difference in configurational entropy between the metastable skyrmion state and the saddle point. In the system we studied, the configurational entropy was found to decrease between the metastable state and the saddle point $(\Delta S<0)$, which results in lowered attempt frequencies. This there-

fore constitutes a stabilizing effect, which we have referred to as entropic narrowing in the saddle point region. The strong entropic contribution mainly originates from the skyrmion's internal modes, and is generally more pronounced for collapse mechanisms. One important consequence of this result is that, at room temperature, the most probable mechanism is not necessarily the one with the lowest internal energy barrier, because it may also possess a low attempt frequency. We used material pa- 
rameters of $\mathrm{Pt} / \mathrm{Co} / \mathrm{AlO}_{\mathrm{x}}$ thin films, in which we tuned the effective anisotropy and strength of the DMI to stabilize isolated skyrmions of about $10 \mathrm{~nm}$ in diameter at zero applied magnetic field. The activation barriers were calculated to be around $300 \mathrm{meV}$. At room temperature, the skyrmion was found to be more stable with respect to collapse mechanisms, due to uncharacteristically low attempt frequencies for collapse in the megahertz regime. Far away from edges and structural defects, the average lifetime of the skyrmion against collapse was found to be of the order of a few milliseconds.

Next, we used forward flux sampling, which is a path sampling method for the simulation of rare events, to compute collapse rates of metastable skyrmions in an infinite system. The goal was to compare the FFS results with the ones obtained from Langer's theory. This was an important step, because the use of Langer's theory is based on many assumptions. In particular, it requires the different mechanisms that exist to be independent from one another in order for the individual rates to add up, which may not hold. An overall good agreement was found between Langer's theory and FFS, thus confirming the strong dependence of the attempt frequency on the external field. Within our range of studied applied field values $(0 \leq B \leq 730 \mathrm{mT})$, the attempt frequency for the collapse extends from a few megahertz at zero-field, to a few gigahertz - or a few tens of gigahertz - at higher field, thus spanning over three to four orders of magnitude. This leads to the following conclusion: due to a strong entropic contribution, internal energy barriers are not enough in order to correctly predict the lifetime of magnetic skyrmions, and it is essential to also evaluate a rate prefactor. This constitutes the main result of the present thesis.

Lastly, we looked at paths to annihilation of first- and second-order skyrmions and antiskyrmions on the frustrated $J_{1}-J_{2}-J_{3}$ square lattice. Frustrated exchange was shown to arise at certain types of interfaces by DFT calculations, most notably in $\mathrm{Pd}_{\mathrm{fcc}} / \mathrm{Fe}, \mathrm{Rh} / \mathrm{Fe}$, and $\mathrm{Rh} / \mathrm{Co}$ bilayers on $\operatorname{Ir}(111)$. We found that, under frustrated exchange couplings, and in certain regions of parameter space, the annihilation of skyrmionic solutions no longer occurs through an isotropic type of collapse, and instead involves the injection of the opposite topological charge into the system, by means of the nucleation of merons and antimerons. We added a weak interfacial DMI into the system to break degeneracies, and we found that the antimeron injection was a preferred mechanism for the annihilation of skyrmions in the low DMI and low magnetic field region of parameter space, where antiskyrmion solutions also exist. The injection of the opposite charge usually happens shortly after the system has passed the saddle point configuration, or at the saddle point in the case of the skyrmion, and also coincides with the loss of one unit of topological charge. Alternatively, the secondorder (anti)skyrmion may split into a bound (anti)skyrmion pair, which involves no change in the total topological charge. In all cases, the skyrmions in this particular 
system only span over a few lattice sites and were found to be quite unstable, both in terms of activation energy and rate prefactor. By using the first-neighbour exchange coupling constant and saturation magnetization of $\mathrm{Pt} / \mathrm{Co} / \mathrm{AlO}_{\mathrm{x}}$, internal energy barriers were calculated to be below $200 \mathrm{meV}$ for the skyrmion - or below $50 \mathrm{meV}$ for the antiskyrmion - and the prefactor was found in the range of a few gigahertz to a few thousands of gigahertz. In all cases, the (anti)skyrmion lifetimes at room temperature were under one nanosecond. The most stable skyrmion was found to be the one stabilized at high DMI which annihilates via isotropic collapse. For the system we studied, this implies that the existence of antiskyrmions may be an indicator of lower skyrmion stability. This is not necessarily true in other systems. For instance, in Ref. [118], higher internal energy barriers were typically associated with the antimeron injection, which is preferred over the isotropic collapse at low applied field. However, a prefactor was not computed in Ref. [118].

The existence of a strong entropic contribution to the thermal stability of skyrmions was initially proposed in [157], but the treatment only compared the entropy of the skyrmion state to that of the ferromagnetic state without considering the saddle point configuration, and was therefore incomplete. The following works mentioned below were published over the duration of this thesis. Experimentally, strong entropic effects were observed by Wild et al. in the case of the decay of the skyrmion lattice in the $\mathrm{B} 20$ chiral magnet $\mathrm{Fe}_{1-x} \mathrm{Co}_{\mathrm{x}} \mathrm{Si}[46]$. The authors measured a variation of the attempt frequency over 30 orders of magnitude when the applied field changed by about $20 \mathrm{mT}$. Meanwhile, a similar methodology to the one presented in this thesis was used by Bessarab et al. [63], in which the rate prefactor was calculated through harmonic transition state theory [127]. The aforementioned work proposed a treatment of the translational Goldstone modes of the skyrmion in a case where the metastable skyrmion state decouples from the lattice, which yields a temperature-dependence of the prefactor, as well as a system size-dependence for escape processes. This was not treated in this thesis, in which the coupling of the skyrmion to the underlying lattice breaks the translational symmetry, and leads to lattice effects in the rate prefactor. In line with the above discussion, the different choice of material parameters in Ref. [63], which were obtained via ab initio DFT calculations for $\mathrm{Pd} / \mathrm{Fe} / \mathrm{Ir}(111)$, yielded attempt frequencies and skyrmion lifetimes vastly different from the ones reported here, with attempt frequencies for collapse spanning from tens of gigahertz, to hundreds of terahertz. From what we understand, HTST is similar to Langer's theory and also pertains to the IHD regime. However, it does not explicitly depend on the damping value, and should therefore give an upper bound of the attempt frequency over the range of possible dampings. Interestingly, we have found that skyrmion collapse rates calculated 
with Langer's theory are not strongly affected by the choice of damping, although the theory is, of course, only applicable to the IDH regime. Apart from collapse and escape processes, duplication and merging of skyrmions were also reported as possible transition mechanisms by a mode following method [142]. Lastly, Ref. [109] reported a drastic change in the attempt frequency of up to seven orders of magnitude per change in magnetic field of one tesla for skyrmion collapse, which is consistent with the main results of this thesis. The authors found that, above a certain size of the metastable skyrmion, the entropy difference between the skyrmion and the saddle point seems to decrease linearly (become more negative) with the surface area $r^{2}$ of the skyrmion. For very small skyrmions $\left(r^{2} \lesssim 6 \mathrm{~nm}^{2}\right), \Delta S>0$, thus leading to a destabilizing effect. This confirms the somewhat intuitive result that smaller skyrmions are less stable, which we have also seen in Chapter 4 by varying the destabilizing magnetic field. This is explained by the fact that the internal energy barrier decreases with the skyrmion size (fewer spins to reverse), and, concurrently, the attempt frequency increases under the effect of the entropic contribution.

Technological applications require sub-10-nm skyrmions to be stable at room temperature in order to compete against existing solutions [38,168]. Despite the somewhat pessimistic conclusions drawn above, some other factors may allow for the stabilization of such small skyrmions. Small skyrmions are generally more stable at higher DMI and lower effective anisotropy [169]. Frustrated exchange, which arises from interface effects, was shown to increase the activation energy for skyrmion collapse in $\mathrm{Pd} / \mathrm{Fe} / \mathrm{Ir}(111)$ [165]. In $\mathrm{Rh} / \mathrm{Co} / \mathrm{Ir}(111)$, skyrmions of $5 \mathrm{~nm}$ stabilized at zero magnetic field were recently observed at $4 \mathrm{~K}$ in the virgin FM state via spin-polarized STM [118]. A combination of DFT calculations and atomistic GNEB simulations showed that $\mathrm{Rh} / \mathrm{Co} / \mathrm{Ir}(111)$ exhibits a strong exchange frustration, which in turn plays an important role in the stabilization of small skyrmions. Additionally, one may attempt to tune the different interactions in order to decrease the entropy of the saddle point configuration, thus resulting in a more negative $\Delta S$ and enhancing the effect of entropic narrowing. Last but not least, the prefactor of the skyrmion collapse rate has, so far, only been theoretically predicted in two-dimensional systems. Skyrmion stability should, in principle, increase in multilayers, for the following reasons: (i) the energy barriers increase if the spin texture is homogeneous along $z$ (for a stacking of the layers along the $z$-direction) [105] ; (ii) if the change in entropy scales like the skyrmion volume in three dimensions [109], then the attempt frequencies may decrease even further. To this day, the smallest skyrmions observed at room temperature were 30 to 40 $\mathrm{nm}$ in diameter, and were reported in multilayer asymmetric stackings of magnetic and non-magnetic heavy metal layers [105, 170]. 
Nonetheless, predicting skyrmion stability in multilayers is challenging. With the increase in the system size, the computational cost increases as well. Additionally, dipolar interactions should, in that case, be treated more accurately than via an effective anisotropy approximation [92, 158]. Hybrid chirality effects may arise from the interplay of dipolar interactions and DMI [171] and have complex consequences on the thermal stability. For methods based on reaction rate theory, three-dimensional systems with dipolar interactions require the diagonalization of very large, non-sparse matrices. In direct Langevin or path sampling simulations, the cost - and therefore the CPU time - of each iteration increases a lot. These issues will require innovative solutions, such as analytical models that may enable the fitting of the ratio of eigenvalues [62] in large systems, combining existing methods, or a different class of methods altogether.

In the past, estimating the stability of magnetic structures has often been synonymous with estimating internal energy barriers, while assuming a typically constant value of the prefactor within the gigahertz range [64-67]. Hence, it has been well accepted within the magnetism community that aiming for internal energy barriers of the order of $40-50 k_{B} T$ at room temperature is a sufficient and necessary condition in order to achieve the desired 10-year stability of magnetic storage bits. This is no longer valid in the case of magnetic skyrmions, and the search for small, room-temperature stable skyrmions must also necessarily involve some "entropic engineering" to tune the attempt frequency, in addition to the activation barrier.

Finally, concerning skyrmion activation rates, some problems are yet to be tackled. One of them is the rate of thermally activated skyrmion nucleation. Although the energy barriers to skyrmion nucleation tend to be much larger than that of annihilation, one could, using entropy arguments, expect much larger attempt frequencies [157]. While the scope of Langer's theory is in principle limited to the decay rates of metastable states, nucleation rates can be obtained with a tool such as forward flux sampling. Another problem is the effect of current-induced spin transfer torques on skyrmion stability. This is relevant to potential technological applications, since skyrmions should be moveable information bits. Under spin transfer torques, skyrmions are deformed compared to their static profile [172]. This may have some important effects on the skyrmion stability.

In fine, magnetic skyrmion may, or may not, constitute the future of magnetic data storage. Nevertheless, with their unique properties, they remain a rich and fascinating topic for fundamental research. 


\section{Appendices}





\section{Appendix A}

\section{Numerical accuracy in Langer's theory implementation}

\section{A.1 Checking for numerical noice: influence of the lat- tice size}

It should be noted that numerical rounding errors can affect the accuracy of the ratio of eigenvalues, as previously mentioned in Ref. [62]. In Table A.1, we gather results of simulations performed for different lattice sizes. For processes involving a single skyrmion and $30 \times 30$ simulated sites, the skyrmion is confined by the boundary and less stable. However, at $50 \times 50$ sites and above, we observe very little variations in the different terms of the prefactor. In the case of the two-skyrmion process, the rate loses its lattice-size dependence for $70 \times 70$ simulated sites and above. If significant numerical noise were contained within the ratio of eigenvalues, the prefactor would change when varying the number of simulated sites in the system. This does not seem to be the case here, which increases confidence in the present results.

\section{A.2 Influence of the tolerance on the GNEB force}

When the order of the saddle point involved in a particular mechanism is higher than one, the CI-GNEB scheme will usually make the path change to a different path involving a first-order SP. This is the case even when the path was previously well relaxed to a low tolerance by the GNEB scheme without a climbing image. This occurs because the $\mathrm{CI}$ is only designed to climb uphill along the reaction coordinate. If other degrees of freedom are unstable at the SP, the CI will move downhill along these directions, and will therefore usually not converge to a higher-order SP. However, we might still want to estimate the contribution of paths involving higher-order SPs to the total rate. In 
(a) Collapse (mechanism $\left.\mathrm{n}^{0} 1\right)$.

\begin{tabular}{ccccc}
\hline \hline$N$ & $\Delta E\left(J_{\text {ex }}\right)$ & $\Omega_{0}\left(\times 10^{-5}\right)$ & $\lambda_{+}(\mathrm{GHz})$ & $f_{0}(\mathrm{MHz})$ \\
\hline $30 \times 30$ & 2.76 & 3.79 & 1198.73 & 7.24 \\
$50 \times 50$ & 2.83 & 3.51 & 1200.47 & 6.70 \\
$70 \times 70$ & 2.83 & 3.49 & 1200.48 & 6.67 \\
\hline \hline
\end{tabular}

(b) Escape (flat boundary).

\begin{tabular}{ccccc}
\hline \hline$N$ & $\Delta E\left(J_{\mathrm{ex}}\right)$ & $\Omega_{0}\left(\times 10^{-2}\right)$ & $\lambda_{+}(\mathrm{GHz})$ & $f_{0}(\mathrm{GHz})$ \\
\hline $30 \times 30$ & 3.23 & 1.20 & 521.60 & 3.80 \\
$50 \times 50$ & 3.28 & 1.24 & 522.94 & 4.14 \\
$70 \times 70$ & 3.28 & 1.24 & 522.94 & 4.13 \\
\hline \hline
\end{tabular}

(c) 2 sk. collapse.

\begin{tabular}{ccccc}
\hline \hline$N$ & $\Delta E\left(J_{\text {ex }}\right)$ & $\Omega_{0}\left(\times 10^{-5}\right)$ & $\lambda_{+}(\mathrm{GHz})$ & $f_{0}(\mathrm{MHz})$ \\
\hline $50 \times 50$ & 2.82 & 1.86 & 1198.11 & 3.56 \\
$60 \times 60$ & 2.82 & 2.49 & 1200.22 & 4.76 \\
$70 \times 70$ & 2.82 & 2.33 & 1200.23 & 4.44 \\
$80 \times 80$ & 2.82 & 2.32 & 1200.23 & 4.43 \\
\hline \hline
\end{tabular}

Table A.1: Terms of the rate prefactor calculated for different number of sites $N=$ $N_{x} \times N_{y}\left(\right.$ here $\left.N_{x}=N_{y}\right)$.

\begin{tabular}{cccccc}
\hline \hline$F_{\text {tol }}$ & $\Delta E\left(J_{\mathrm{ex}}\right)$ & $\Omega_{0}\left(\times 10^{-5}\right)$ & $\lambda_{+}(\mathrm{GHz})$ & $f_{0}(\mathrm{MHz})$ & $\begin{array}{c}\tau(300 \mathrm{~K}) \\
(\mathrm{ms})\end{array}$ \\
\hline $1 \times 10^{-12}$ & 2.8256343320 & 3.51 & 1200.473 & 6.70 & 8.2 \\
$6 \times 10^{-5}$ & 2.8256343324 & 3.76 & 1200.474 & 7.18 & 7.7 \\
\hline \hline
\end{tabular}

Table A.3: For collapse mechanism $\mathrm{n}^{0} 1$, energy barrier, terms in the rate prefactor and lifetime comparison for different values of the set tolerance on the (CI)-GNEB force.

that case, we have to set a lower tolerance on the GNEB force, so that the scheme will terminate before the path changes. In Table A.3 we compare the energy barrier and the terms of the prefactor for low and high tolerance on the GNEB force in the case of collapse mechanism $n^{o} 1$. Although we lose some accuracy in the rate, the results still remain close so that, on the scale of skyrmion stability, the skyrmion lifetime obtained at higher tolerance is still acceptable. Alternatively, one could interpolate the energy in the vicinity of the SP with a high enough density of interpolation points, and choose the highest energy point as the SP. 


\section{Bibliography}

[1] L. Desplat, D. Suess, J.-V. Kim, and R. L. Stamps. Thermal stability of metastable magnetic skyrmions: Entropic narrowing and significance of internal eigenmodes. Physical Review B, 98:134407, 2018.

[2] L. Desplat, J.-V. Kim, and R. L. Stamps. Paths to annihilation of first-and secondorder (anti) skyrmions via (anti) meron nucleation on the frustrated square lattice. Physical Review B, 99(17):174409, 2019.

[3] L. Desplat, C. Vogler, J.-V. Kim, R. L. Stamps, and D. Suess. Path sampling for lifetimes of metastable magnetic skyrmions and direct comparison with Kramers' method. arXiv:1905.04110, 2019.

[4] J. S. Russell. Report on waves. In 14th meeting of the British Association for the Advancement of Science, pages 90-311, 1844.

[5] P. G. Drazin and R. S. Johnson. Solitons: An introduction. Cambridge University Press, Cambridge, United Kingdom, 1989.

[6] R. Rajaraman. Solitons and Instantons: An introduction to solitons and instantons in quantum field theory. North-Holland, Amsterdam (Netherlands), 1982.

[7] J. Zang, V. Cros, and A. Hoffmann. Topology in Magnetism, volume 192 of Springer Series in Solid-State Sciences. Springer Nature, Switzerland, 2018.

[8] T. H. R. Skyrme. A non-linear field theory. Proceedings of the Royal Society, 260:127, 1961.

[9] A. Bogdanov and D. Yablonskii. Thermodynamically stable "vortices" in magnetically ordered crystals. The mixed state of magnets. Zhurnal Éksperimental'nŏ i Teoreticheskoŭ Fiziki, 95:182, 1989.

[10] B. Ivanov, V. Stephanovich, and A. Zhmudskii. Magnetic vortices - The microscopic analogs of magnetic bubbles. Journal of Magnetism and Magnetic Materials, 88(1-2):116-120, 1990. 
[11] A. Bogdanov and A. Hubert. Thermodynamically stable magnetic vortex states in magnetic crystals. Journal of Magnetism and Magnetic Materials, 138(3):255269, 1994.

[12] I. E. Dzyaloshinskii. A thermodynamic theory of "weak" ferromagnetism of antiferromagnetics. Journal of Physics and Chemistry of Solids, 4(4):241-255, 1958.

[13] T. Moriya. Anisotropic superexchange interaction and weak ferromagnetism. Physical Review, 120(1):91, 1960.

[14] A. Fert. Magnetic and transport properties of metallic multilayers. In Materials Science Forum, volume 59, pages 439-480. Trans Tech Publications, 1990.

[15] A. Crépieux and C. Lacroix. Dzyaloshinsky-Moriya interactions induced by symmetry breaking at a surface. Journal of Magnetism and Magnetic Materials, 182(3):341-349, 1998.

[16] S. Chikazumi and C. D. Graham. Physics of Ferromagnetism, volume 94 of International Series of Monographs on Physics. Oxford University Press, 2nd edition, 2009.

[17] A. Malozemoff and J. Slonczewski. Magnetic Domain Walls in Bubble Materials: Advances in Materials and Device Research, volume 1. Academic press, 1979.

[18] X. Yu, M. Mostovoy, Y. Tokunaga, W. Zhang, K. Kimoto, Y. Matsui, Y. Kaneko, N. Nagaosa, and Y. Tokura. Magnetic stripes and skyrmions with helicity reversals. Proceedings of the National Academy of Sciences, 109(23):8856-8860, 2012.

[19] M. Finazzi, M. Savoini, A. Khorsand, A. Tsukamoto, A. Itoh, L. Duo, A. Kirilyuk, T. Rasing, and M. Ezawa. Laser-induced magnetic nanostructures with tunable topological properties. Physical Review Letters, 110(17):177205, 2013.

[20] M. Nagao, Y.-G. So, H. Yoshida, M. Isobe, T. Hara, K. Ishizuka, and K. Kimoto. Direct observation and dynamics of spontaneous skyrmion-like magnetic domains in a ferromagnet. Nature Nanotechnology, 8(5):325, 2013.

[21] W. Koshibae and N. Nagaosa. Theory of antiskyrmions in magnets. Nature Communications, 7:10542, 2016.

[22] A. Leonov and M. Mostovoy. Multiply periodic states and isolated skyrmions in an anisotropic frustrated magnet. Nature Communications, 6:8275, 2015.

[23] T. Okubo, S. Chung, and H. Kawamura. Multiple-q states and the skyrmion lattice of the triangular-lattice Heisenberg antiferromagnet under magnetic fields. Physical Review Letters, 108(1):017206, 2012. 
[24] N. Nagaosa and Y. Tokura. Topological properties and dynamics of magnetic skyrmions. Nature Nanotechnology, 8(12):899, 2013.

[25] S.-Z. Lin and S. Hayami. Ginzburg-Landau theory for skyrmions in inversion-symmetric magnets with competing interactions. Physical Review B, 93(6):064430, 2016.

[26] L. Rózsa, K. Palotás, A. Deák, E. Simon, R. Yanes, L. Udvardi, L. Szunyogh, and U. Nowak. Formation and stability of metastable skyrmionic spin structures with various topologies in an ultrathin film. Physical Review B, 95(9):094423, 2017.

[27] X. Zhang, J. Xia, Y. Zhou, X. Liu, H. Zhang, and M. Ezawa. Skyrmion dynamics in a frustrated ferromagnetic film and current-induced helicity locking-unlocking transition. Nature Communications, 8(1):1717, 2017.

[28] S. Mühlbauer, B. Binz, F. Jonietz, C. Pfleiderer, A. Rosch, A. Neubauer, R. Georgii, and P. Böni. Skyrmion lattice in a chiral magnet. Science, 323(5916):915-919, 2009.

[29] X. Yu, Y. Onose, N. Kanazawa, J. Park, J. Han, Y. Matsui, N. Nagaosa, and Y. Tokura. Real-space observation of a two-dimensional skyrmion crystal. Nature, 465(7300):901, 2010.

[30] Y. Tokunaga, X. Yu, J. White, H. M. Rønnow, D. Morikawa, Y. Taguchi, and Y. Tokura. A new class of chiral materials hosting magnetic skyrmions beyond room temperature. Nature Communications, 6:7638, 2015.

[31] A. K. Nayak, V. Kumar, T. Ma, P. Werner, E. Pippel, R. Sahoo, F. Damay, U. K. Rößler, C. Felser, and S. S. Parkin. Magnetic antiskyrmions above room temperature in tetragonal Heusler materials. Nature, 548(7669):561, 2017.

[32] N. Kanazawa, S. Seki, and Y. Tokura. Noncentrosymmetric magnets hosting magnetic skyrmions. Advanced Materials, 29(25):1603227, 2017.

[33] N. Romming, C. Hanneken, M. Menzel, J. E. Bickel, B. Wolter, K. von Bergmann, A. Kubetzka, and R. Wiesendanger. Writing and deleting single magnetic skyrmions. Science, 341(6146):636-639, 2013.

[34] S. Heinze, K. Von Bergmann, M. Menzel, J. Brede, A. Kubetzka, R. Wiesendanger, G. Bihlmayer, and S. Blügel. Spontaneous atomic-scale magnetic skyrmion lattice in two dimensions. Nature Physics, 7(9):713, 2011.

[35] S. S. Parkin, M. Hayashi, and L. Thomas. Magnetic domain-wall racetrack memory. Science, 320(5873):190-194, 2008. 
[36] J. Sampaio, V. Cros, S. Rohart, A. Thiaville, and A. Fert. Nucleation, stability and current-induced motion of isolated magnetic skyrmions in nanostructures. Nature Nanotechnology, 8(11):839, 2013.

[37] S. Woo, K. Litzius, B. Krüger, M.-Y. Im, L. Caretta, K. Richter, M. Mann, A. Krone, R. M. Reeve, M. Weigand, et al. Observation of room-temperature magnetic skyrmions and their current-driven dynamics in ultrathin metallic ferromagnets. $\mathrm{Na}$ ture Materials, 15(5):501, 2016.

[38] A. Fert, V. Cros, and J. Sampaio. Skyrmions on the track. Nature Nanotechnology, 8(3):152, 2013.

[39] J. Iwasaki, M. Mochizuki, and N. Nagaosa. Current-induced skyrmion dynamics in constricted geometries. Nature Nanotechnology, 8(10):742, 2013.

[40] Y. Zhou and M. Ezawa. A reversible conversion between a skyrmion and a domain-wall pair in a junction geometry. Nature Communications, 5:4652, 2014.

[41] J. Müller. Magnetic skyrmions on a two-lane racetrack. New Journal of Physics, 19(2):025002, 2017.

[42] P.-J. Hsu, A. Kubetzka, A. Finco, N. Romming, K. von Bergmann, and R. Wiesendanger. Electric-field-driven switching of individual magnetic skyrmions. $\mathrm{Na}$ ture Nanotechnology, 12(2):123, 2017.

[43] B. Dupé, M. Hoffmann, C. Paillard, and S. Heinze. Tailoring magnetic skyrmions in ultra-thin transition metal films. Nature Communications, 5:4030, 2014.

[44] B. Dupé, G. Bihlmayer, M. Böttcher, S. Blügel, and S. Heinze. Engineering skyrmions in transition-metal multilayers for spintronics. Nature Communications, 7:11779, 2016.

[45] H.-B. Braun. Topological effects in nanomagnetism: from superparamagnetism to chiral quantum solitons. Advances in Physics, 61(1):1-116, 2012.

[46] J. Wild, T. N. Meier, S. Pöllath, M. Kronseder, A. Bauer, A. Chacon, M. Halder, M. Schowalter, A. Rosenauer, J. Zweck, et al. Entropy-limited topological protection of skyrmions. Science Advances, 3(9):e1701704, 2017.

[47] W. Coffey, D. Garanin, and D. McCarthy. Crossover formulas in the Kramers theory of thermally activated escape rates-Application to spin systems. Advances in Chemical Physics, 117:483-765, 2001. 
[48] H. Risken. The Fokker-Planck Equation, volume 18 of Springer Series in Synergetic. Springer, Berlin, Heidelberg, 1996.

[49] J. S. Langer. Statistical theory of the decay of metastable states. Annals of Physics, 54:258-275, 1969.

[50] P. Hänggi, P. Talkner, and M. Borkovec. Reaction-rate theory: Fifty years after Kramers. Reviews of Modern Physics, 62(2):251, 1990.

[51] W. T. Coffey and Y. P. Kalmykov. Thermal fluctuations of magnetic nanoparticles: Fifty years after Brown. Journal of Applied Physics, 112(12):121301, 2012.

[52] H.-B. Braun. Kramers's rate theory, broken symmetries, and magnetization reversal. Journal of Applied Physics, 76(10):6310-6315, 1994.

[53] P. N. Loxley and R. L. Stamps. Theory for nucleation at an interface and magnetization reversal of a two-layer nanowire. Physical Review B, 73(2):024420, 2006.

[54] P. B. Visscher and R. Zhu. Low-dimensionality energy landscapes: Magnetic switching mechanisms and rates. Physica B: Condensed Matter, 407(9):13401344, 2012.

[55] G. Mills, H. Jónsson, and G. K. Schenter. Reversible work transition state theory: application to dissociative adsorption of hydrogen. Surface Science, 324(2-3):305337, 1995.

[56] H. Jónsson, G. Mills, and K. W. Jacobsen. Nudged elastic band method for finding minimum energy paths of transitions. In Classical and Quantum Dynamics in Condensed Phase Simulations, pages 385-404, 1998.

[57] R. Dittrich, T. Schrefl, D. Suess, W. Scholz, H. Forster, and J. Fidler. A path method for finding energy barriers and minimum energy paths in complex micromagnetic systems. Journal of Magnetism and Magnetic Materials, 250:12-19, 2002.

[58] G. Henkelman, B. P. Uberuaga, and H. Jónsson. A climbing image nudged elastic band method for finding saddle points and minimum energy paths. The Journal of Chemical Physics, 113(22):9901-9904, 2000.

[59] P. F. Bessarab, V. M. Uzdin, and H. Jonsson. Method for finding mechanism and activation energy of magnetic transitions, applied to skyrmions and antivortex annihilation. Computer Physics Communications, 196:335-347, November 2015. 
[60] E. Weinan, W. Ren, and E. Vanden-Eijnden. String method for the study of rare events. Physical Review B, 66(5):052301, 2002.

[61] W. E, W. Ren, and E. Vanden-Eijnden. Simplified and improved string method for computing the minimum energy paths in barrier-crossing events. The Journal of Chemical Physics, 126(16):164103, 2007.

[62] G. Fiedler, J. Fidler, J. Lee, T. Schrefl, R. L. Stamps, H. Braun, and D. Suess. Direct calculation of the attempt frequency of magnetic structures using the finite element method. Journal of Applied Physics, 111(9):093917, 2012.

[63] P. F. Bessarab, G. P. Müller, I. S. Lobanov, F. N. Rybakov, N. S. Kiselev, H. Jónsson, V. M. Uzdin, S. Blügel, L. Bergqvist, and A. Delin. Annihilation mechanisms and lifetime of racetrack skyrmions. Scientific Reports, 8(3433):618, 2018.

[64] D. Weller and A. Moser. Thermal effect limits in ultrahigh-density magnetic recording. IEEE Transactions on Magnetics, 35(6):4423-4439, 1999.

[65] E. Chen, D. Apalkov, Z. Diao, A. Driskill-Smith, D. Druist, D. Lottis, V. Nikitin, X. Tang, S. Watts, S. Wang, et al. Advances and future prospects of spin-transfer torque random access memory. IEEE Transactions on Magnetics, 46(6):1873-1878, 2010.

[66] M. Lederman, S. Schultz, and M. Ozaki. Measurement of the dynamics of the magnetization reversal in individual single-domain ferromagnetic particles. Physical Review Letters, 73(14):1986, 1994.

[67] D. Cortés-Ortuño, W. Wang, M. Beg, R. A. Pepper, M.-A. Bisotti, R. Carey, M. Vousden, T. Kluyver, O. Hovorka, and H. Fangohr. Thermal stability and topological protection of skyrmions in nanotracks. Scientific Reports, 7(1):4060, 2017.

[68] J. L. García-Palacios and F. J. Lázaro. Langevin-dynamics study of the dynamical properties of small magnetic particles. Physical Review B, 58(22):14937, 1998.

[69] A. F. Voter. Hyperdynamics: Accelerated molecular dynamics of infrequent events. Physical Review Letters, 78(20):3908, 1997.

[70] J. Xue and R. Victora. Micromagnetic predictions for thermally assisted reversal over long time scales. Applied Physics Letters, 77(21):3432-3434, 2000.

[71] C. Dellago, P. G. Bolhuis, F. S. Csajka, and D. Chandler. Transition path sampling and the calculation of rate constants. The Journal of Chemical Physics, 108(5):1964-1977, 1998. 
[72] P. G. Bolhuis, D. Chandler, C. Dellago, and P. L. Geissler. Transition path sampling: Throwing ropes over rough mountain passes, in the dark. Annual Review of Physical Chemistry, 53(1):291-318, 2002.

[73] T. S. van Erp, D. Moroni, and P. G. Bolhuis. A novel path sampling method for the calculation of rate constants. The Journal of Chemical Physics, 118(17):7762-7774, 2003.

[74] R. J. Allen, P. B. Warren, and P. R. ten Wolde. Sampling Rare Switching Events in Biochemical Networks. Physical Review Letters, 94:018104, Jan 2005.

[75] R. J. Allen, D. Frenkel, and P. R. ten Wolde. Simulating rare events in equilibrium or nonequilibrium stochastic systems. The Journal of Chemical Physics, 124(2):024102, 2006.

[76] R. J. Allen, D. Frenkel, and P. R. ten Wolde. Forward flux sampling-type schemes for simulating rare events: Efficiency analysis. The Journal of Chemical Physics, 124(19):194111, 2006.

[77] R. J. Allen, C. Valeriani, and P. R. ten Wolde. Forward flux sampling for rare event simulations. Journal of Physics: Condensed Matter, 21(46):463102, 2009.

[78] C. Vogler, F. Bruckner, B. Bergmair, T. Huber, D. Suess, and C. Dellago. Simulating rare switching events of magnetic nanostructures with forward flux sampling. Physical Review B, 88(13):134409, 2013.

[79] C. Vogler, F. Bruckner, D. Suess, and C. Dellago. Calculating thermal stability and attempt frequency of advanced recording structures without free parameters. Journal of Applied Physics, 117(16):163907, 2015.

[80] S. Rohart, J. Miltat, and A. Thiaville. Path to collapse for an isolated Néel skyrmion. Physical Review B, 93(21):214412, 2016.

[81] D. Suess, C. Vogler, F. Bruckner, P. Heistracher, and C. Abert. A repulsive skyrmion chain as a guiding track for a racetrack memory. AIP Advances, 8(11):115301, 2018.

[82] H. A. Kramers. Brownian motion in a field of force and the diffusion model of chemical reactions. Physica, 7(4):284-304, 1940.

[83] D. Craik. Magnetism: Principles and Applications. Wiley, 1995.

[84] L. Landau and E. Lifshitz. Domain walls. Physikalische Zeitschrift der Sowjetunion, $8: 153,1935$. 
[85] W. Heisenberg. Zür theorie des ferromagnetismus. Zeitschrift für Physik A Hadrons and Nuclei, 49:619-636, 1928.

[86] P. A. M. Dirac. Quantum mechanics of many-electron systems. Proceedings of the Royal Society of London. Series A, Containing Papers of a Mathematical and Physical Character, 123(792):714-733, 1929.

[87] Y. Ishikawa, K. Tajima, D. Bloch, and M. Roth. Helical spin structure in manganese silicide MnSi. Solid State Communications, 19(6):525-528, 1976.

[88] Y. Ishikawa, G. Shirane, J. Tarvin, and M. Kohgi. Magnetic excitations in the weak itinerant ferromagnet MnSi. Physical Review B, 16(11):4956, 1977.

[89] A. Bogdanov and U. Rößler. Chiral symmetry breaking in magnetic thin films and multilayers. Physical Review Letters, 87(3):037203, 2001.

[90] C. Kittel. Introduction to Solid State Physics. Wiley, 7th edition, 1996.

[91] A. Hubert and R. Schäfer. Magnetic domains: the analysis of magnetic microstructures. Springer, Berlin Heidelberg, Germany, 2008.

[92] J. M. Winter. Bloch Wall Excitation. Application to Nuclear Resonance in a Bloch Wall. Physical Review, 124:452-459, Oct 1961.

[93] F. Bloch. Zur Theorie des Austauschproblems und der Remanenzerscheinung der Ferromagnetika [On the theory of the exchange problem and the remanence phenomenon of ferromagnets]. Zeitschrift für Physik, 74:295, 1932.

[94] S. Tarasenko, A. Stankiewicz, V. Tarasenko, and J. Ferré. Bloch wall dynamics in ultrathin ferromagnetic films. Journal of Magnetism and Magnetic Materials, 189(1):19-24, 1998.

[95] M. Heide, G. Bihlmayer, and S. Blügel. Dzyaloshinskii-Moriya interaction accounting for the orientation of magnetic domains in ultrathin films: Fe/W (110). Physical Review B, 78(14):140403, 2008.

[96] A. Thiaville, S. Rohart, É. Jué, V. Cros, and A. Fert. Dynamics of Dzyaloshinskii domain walls in ultrathin magnetic films. Europhysics Letters, 100(5):57002, 2012.

[97] H. B. Braun. Fluctuations and instabilities of ferromagnetic domain-wall pairs in an external magnetic field. Physical Review B, 50(22):16485, 1994. 
[98] B. M. Piette, B. J. Schroers, and W. Zakrzewski. Multisolitons in a twodimensional Skyrme model. Zeitschrift für Physik C Particles and Fields, 65(1):165-174, 1995.

[99] T. Weidig. The baby Skyrme models and their multi-skyrmions. Nonlinearity, 12(6):1489, 1999.

[100] A. S. Schwarz. Topology for physicists, volume 308 of A Series of Comprehensive Studies in Mathematics. Springer, Berlin Heidelberg, Germany, 2013.

[101] A. Belavin and A. Polyakov. Metastable states of two-dimensional isotropic ferromagnets. JETP Letters, 22(10):245-248, 1975.

[102] C. Heo, N. S. Kiselev, A. K. Nandy, S. Blügel, and T. Rasing. Switching of chiral magnetic skyrmions by picosecond magnetic field pulses via transient topological states. Scientific Reports, 6:27146, 2016.

[103] B. Berg and M. Lüscher. Definition and statistical distributions of a topological number in the lattice O (3) $\sigma$-model. Nuclear Physics B, 190(2):412-424, 1981.

[104] M. Böttcher, S. Heinze, S. Egorov, J. Sinova, and B. Dupé. B-T phase diagram of $\mathrm{Pd} / \mathrm{Fe} / \mathrm{Ir}$ (111) computed with parallel tempering Monte Carlo. New Journal of Physics, 20(10):103014, 2018.

[105] C. Moreau-Luchaire, C. Moutafis, N. Reyren, J. Sampaio, C. Vaz, N. Van Horne, K. Bouzehouane, K. Garcia, C. Deranlot, P. Warnicke, et al. Additive interfacial chiral interaction in multilayers for stabilization of small individual skyrmions at room temperature. Nature Nanotechnology, 11(5):444, 2016.

[106] I. M. Miron, T. Moore, H. Szambolics, L. D. Buda-Prejbeanu, S. Auffret, B. Rodmacq, S. Pizzini, J. Vogel, M. Bonfim, A. Schuhl, et al. Fast current-induced domain-wall motion controlled by the Rashba effect. Nature Materials, 10(6):419, 2011 .

[107] S. Rohart and A. Thiaville. Skyrmion confinement in ultrathin film nanostructures in the presence of Dzyaloshinskii-Moriya interaction. Physical Review B, 88(18):184422, 2013.

[108] N. Romming, A. Kubetzka, C. Hanneken, K. von Bergmann, and R. Wiesendanger. Field-dependent size and shape of single magnetic skyrmions. Physical Review Letters, 114(17):177203, 2015.

[109] S. von Malottki, P. F. Bessarab, S. Haldar, A. Delin, and S. Heinze. Skyrmion lifetime in ultrathin films. Physical Review B, 99(6):060409, 2019. 
[110] S. Banerjee, J. Rowland, O. Erten, and M. Randeria. Enhanced stability of skyrmions in two-dimensional chiral magnets with Rashba spin-orbit coupling. Physical Review X, 4(3):031045, 2014.

[111] M. Hoffmann, B. Zimmermann, G. P. Müller, D. Schürhoff, N. S. Kiselev, C. Melcher, and S. Blügel. Antiskyrmions stabilized at interfaces by anisotropic Dzyaloshinskii-Moriya interactions. Nature Communications, 8(1):308, 2017.

[112] L. Camosi, S. Rohart, O. Fruchart, S. Pizzini, M. Belmeguenai, Y. Roussigné, A. Stashkevich, S. M. Cherif, L. Ranno, M. De Santis, and J. Vogel. Anisotropic dzyaloshinskii-moriya interaction in ultrathin epitaxial au/co/w (110). Physical Review B, 95(21):214422, 2017.

[113] F. N. Rybakov and N. S. Kiselev. Chiral magnetic skyrmions with arbitrary topological charge. Physical Review B, 99(6):064437, 2019.

[114] N. Kiselev, A. Bogdanov, R. Schäfer, and U. Rößler. Chiral skyrmions in thin magnetic films: new objects for magnetic storage technologies? Journal of Physics D: Applied Physics, 44(39):392001, 2011.

[115] G. Butenko. Phenomenological theory of chiral states in magnets with Dzyaloshinskii-Moriya interactions. PhD thesis, Fakultät Mathematik und Naturwissenschaften der Technischen Universität Dresden, 2012.

[116] A. Kubetzka, O. Pietzsch, M. Bode, and R. Wiesendanger. Spin-polarized scanning tunneling microscopy study of 360 walls in an external magnetic field. Physical Review B, 67(2):020401, 2003.

[117] N. Romming, H. Pralow, A. Kubetzka, M. Hoffmann, S. von Malottki, S. Meyer, B. Dupé, R. Wiesendanger, K. von Bergmann, and S. Heinze. Competition of Dzyaloshinskii-Moriya and Higher-Order Exchange Interactions in $\mathrm{Rh} / \mathrm{Fe}$ Atomic Bilayers on Ir(111). Phys. Rev. Lett., 120:207201, May 2018.

[118] S. Meyer, M. Perini, S. von Malottki, A. Kubetzka, R. Wiesendanger, K. von Bergmann, and S. Heinze. Isolated zero field sub-10 nm skyrmions in ultrathin Co films. Nature Communications, (10):3823, 2019.

[119] T. Garel and S. Doniach. Phase transitions with spontaneous modulation-the dipolar Ising ferromagnet. Physical Review B, 26(1):325, 1982.

[120] W. Coffey and Y. P. Kalmykov. The Langevin equation: With applications to stochastic problems in physics, chemistry and electrical engineering, volume 27 . World Scientific, 2012. 
[121] A. Einstein. Über die von der molekularkinetischen Theorie der Wärme geforderte Bewegung von in ruhenden Flüssigkeiten suspendierten Teilchen [Investigations on the theory of Brownian Movement]. Annalen der Physik, 17(8):549-560, 1905.

[122] R. Kubo. The fluctuation-dissipation theorem. Reports on progress in physics, 29(1):255, 1966.

[123] P. Langevin. Sur la Théorie de Mouvement Brownien. Comptes Rendus Académie des Sciences Paris, 146:530-533, 1908.

[124] R. Becker and W. Döring. Kinetische Behandlung der Keimbildung in übersättigten Dämpfen. Annalen der Physik, 416(8):719-752, 1935.

[125] G. Duff. Langer's method for the calculation of escape rates and its application to systems of ferromagnets. PhD thesis, Dublin Institute of Technology, 2008.

[126] P. Loxley. Nucleation of solitons in the presence of defects. PhD thesis, University of Western Australia, 2005.

[127] P. F. Bessarab, V. M. Uzdin, and H. Jónsson. Harmonic transition-state theory of thermal spin transitions. Physica Review B, 85:184409, May 2012.

[128] Private communication.

[129] G. Henkelman and H. Jónsson. Improved tangent estimate in the nudged elastic band method for finding minimum energy paths and saddle points. The Journal of chemical physics, 113(22):9978-9985, 2000.

[130] A. Aharoni. Introduction to the Theory of Ferromagnetism, volume 109 of International Series of Monographs on Physics. Clarendon Press, Oxford, 2000.

[131] W. C. Swope, H. C. Andersen, P. H. Berens, and K. R. Wilson. A computer simulation method for the calculation of equilibrium constants for the formation of physical clusters of molecules: Application to small water clusters. The Journal of Chemical Physics, 76(1):637-649, 1982.

[132] G. P. Müller, M. Hoffmann, C. Dißelkamp, D. Schürhoff, S. Mavros, M. Sallermann, N. S. Kiselev, H. Jónsson, and S. Blügel. Spirit: Multifunctional framework for atomistic spin simulations. Physical Review B, 99(22):224414, 2019.

[133] M. Galassi, J. Davies, J. Theiler, B. Gough, G. Jungman, P. Alken, M. Booth, F. Rossi, and R. Ulerich. GNU Scientific Library. Network Theory Limited, 2002. 
[134] Y. Qiu, G. Guennebaud, and J. Niesen. Spectra: C++ library for large scale eigenvalue problems, 2015.

[135] G. Guennebaud, B. Jacob, et al. Eigen v3. http://eigen.tuxfamily.org, 2010.

[136] A. Vansteenkiste, J. Leliaert, M. Dvornik, M. Helsen, F. Garcia-Sanchez, and B. Van Waeyenberge. The design and verification of MuMax3. AIP Advances, 4(10):107133, 2014.

[137] J. Schratzberger, J. Lee, M. Fuger, J. Fidler, G. Fiedler, T. Schrefl, and D. Suess. Validation of the transition state theory with Langevin-dynamics simulations. Journal of Applied Physics, 108(3):033915, 2010.

[138] P. Heistracher, C. Abert, F. Bruckner, C. Vogler, and D. Suess. GPU-accelerated atomistic energy barrier calculations of skyrmion annihilations. IEEE Transactions on Magnetics, 54(11):1-5, 2018.

[139] U. Ritzmann, S. Von Malottki, J.-V. Kim, S. Heinze, J. Sinova, and B. Dupé. Trochoidal motion and pair generation in skyrmion and antiskyrmion dynamics under spin-orbit torques. Nature Electronics, 1(8):451, 2018.

[140] C. Hanneken, A. Kubetzka, K. Von Bergmann, and R. Wiesendanger. Pinning and movement of individual nanoscale magnetic skyrmions via defects. New Journal of Physics, 18(5):055009, 2016.

[141] V. M. Uzdin, M. N. Potkina, I. S. Lobanov, P. F. Bessarab, and H. Jónsson. The effect of confinement and defects on the thermal stability of skyrmions. Physica B: Condensed Matter, 549:6-9, 2017.

[142] G. P. Müller, P. F. Bessarab, S. M. Vlasov, F. Lux, N. S. Kiselev, S. Blügel, V. M. Uzdin, and H. Jónsson. Duplication, Collapse, and Escape of Magnetic Skyrmions Revealed Using a Systematic Saddle Point Search Method. Physical Review Letters, 121(19):197202, 2018.

[143] C. Schütte, J. Iwasaki, A. Rosch, and N. Nagaosa. Inertia, diffusion, and dynamics of a driven skyrmion. Physical Review B, 90(17):174434, 2014.

[144] I. Makhfudz, B. Krüger, and O. Tchernyshyov. Inertia and chiral edge modes of a skyrmion magnetic bubble. Physical Review Letters, 109(21):217201, 2012.

[145] S.-Z. Lin, C. D. Batista, and A. Saxena. Internal modes of a skyrmion in the ferromagnetic state of chiral magnets. Physical Review B, 89(2):024415, 2014. 
[146] C. Schütte and M. Garst. Magnon-skyrmion scattering in chiral magnets. Physical Review B, 90(9):094423, 2014.

[147] J. Iwasaki, A. J. Beekman, and N. Nagaosa. Theory of magnon-skyrmion scattering in chiral magnets. Physical Review B, 89(6):064412, 2014.

[148] F. Buijnsters, A. Fasolino, and M. Katsnelson. Zero modes in magnetic systems: General theory and an efficient computational scheme. Physical Review B, 89(17):174433, 2014.

[149] S. Schroeter and M. Garst. Scattering of high-energy magnons off a magnetic skyrmion. Low Temperature Physics, 41(10):817-825, 2015.

[150] V. L. Zhang, C. G. Hou, K. Di, H. S. Lim, S. C. Ng, S. D. Pollard, H. Yang, and M. H. Kuok. Eigenmodes of Néel skyrmions in ultrathin magnetic films. AIP Advances, 7(5):055212, 2017.

[151] M. Mruczkiewicz, M. Krawczyk, and K. Y. Guslienko. Spin excitation spectrum in a magnetic nanodot with continuous transitions between the vortex, Blochtype skyrmion, and Néel-type skyrmion states. Physical Review B, 95(9):094414, 2017.

[152] K. Y. Guslienko and Z. V. Gareeva. Gyrotropic skyrmion modes in ultrathin magnetic circular dots. IEEE Magnetics Letters, 8:1-5, 2017.

[153] M. Garst, J. Waizner, and D. Grundler. Collective spin excitations of helices and magnetic skyrmions: review and perspectives of magnonics in noncentrosymmetric magnets. Journal of Physics D: Applied Physics, 50(29):293002, 2017.

[154] V. P. Kravchuk, D. D. Sheka, U. K. Rößler, J. van den Brink, and Y. Gaididei. Spin eigenmodes of magnetic skyrmions and the problem of the effective skyrmion mass. Physical Review B, 97(6):064403, 2018.

[155] A. Bogdanov and A. Hubert. The properties of isolated magnetic vortices. Physica Status Solidi B, 186(2):527-543, 1994.

[156] M. N. Wilson, A. B. Butenko, A. N. Bogdanov, and T. L. Monchesky. Chiral skyrmions in cubic helimagnet films: The role of uniaxial anisotropy. Phys. Rev. B, 89:094411, Mar 2014.

[157] J. Hagemeister, N. Romming, K. Von Bergmann, E. Vedmedenko, and R. Wiesendanger. Stability of single skyrmionic bits. Nature Communications, 6:8455, 2015. 
[158] I. S. Lobanov, H. Jónsson, and V. M. Uzdin. Mechanism and activation energy of magnetic skyrmion annihilation obtained from minimum energy path calculations. Physical Review B, 94(17):174418, 2016.

[159] W. F. Brown Jr. Thermal fluctuations of a single-domain particle. Physical Review, 130(5):1677, 1963.

[160] M. Matsumoto and T. Nishimura. Mersenne twister: a 623-dimensionally equidistributed uniform pseudo-random number generator. ACM Transactions on Modeling and Computer Simulation (TOMACS), 8(1):3-30, 1998.

[161] W. Scholz, T. Schrefl, and J. Fidler. Micromagnetic simulation of thermally activated switching in fine particles. Journal of Magnetism and Magnetic Materials, 233(3):296-304, 2001.

[162] E. E. Borrero and F. A. Escobedo. Simulating the kinetics and thermodynamics of transitions via forward flux/umbrella sampling. The Journal of Physical Chemistry B, 113(18):6434-6445, 2009.

[163] E. E. Borrero and F. A. Escobedo. Optimizing the sampling and staging for simulations of rare events via forward flux sampling schemes. The Journal of Chemical Physics, 129(2):024115, 2008.

[164] Y. P. Kalmykov. The relaxation time of the magnetization of uniaxial singledomain ferromagnetic particles in the presence of a uniform magnetic field. Journal of Applied Physics, 96(2):1138-1145, 2004.

[165] S. von Malottki, B. Dupé, P. Bessarab, A. Delin, and S. Heinze. Enhanced skyrmion stability due to exchange frustration. Scientific Reports, 7(1):12299, 2017.

[166] D. Stosic, J. Mulkers, B. Van Waeyenberge, T. B. Ludermir, and M. V. Milošević. Paths to collapse for isolated skyrmions in few-monolayer ferromagnetic films. Physical Review B, 95(21):214418, 2017.

[167] B. Heil, A. Rosch, and J. Masell. Universality of annihilation barriers of large magnetic skyrmions in chiral and frustrated magnets. Phys. Rev. B, 100:134424, Oct 2019.

[168] A. Fert, N. Reyren, and V. Cros. Magnetic skyrmions: advances in physics and potential applications. Nature Reviews Materials, 2(7):17031, 2017.

[169] W. Legrand. Crafting magnetic skyrmions at room temperature: size, stability and dynamics in multilayers. PhD thesis, Université Paris-Saclay, 2019. 
[170] A. Soumyanarayanan, M. Raju, A. G. Oyarce, A. K. Tan, M.-Y. Im, A. P. Petrović, P. Ho, K. Khoo, M. Tran, C. Gan, et al. Tunable room-temperature magnetic skyrmions in Ir/Fe/Co/Pt multilayers. Nature Materials, 16(9):898, 2017.

[171] W. Legrand, J.-Y. Chauleau, D. Maccariello, N. Reyren, S. Collin, K. Bouzehouane, N. Jaouen, V. Cros, and A. Fert. Hybrid chiral domain walls and skyrmions in magnetic multilayers. Science Advances, 4(7):eaat0415, 2018.

[172] R. E. Troncoso and Á. S. Núñez. Brownian motion of massive skyrmions in magnetic thin films. Annals of Physics, 351:850-856, 2014. 